UNIVERSITY OF CALIFORNIA

IRVINE

\title{
Observation of Relativistic
}

\section{Antihydrogen Atoms}

\author{
DISSERTATION \\ submitted in partial satisfaction of the requirements for the degree of \\ DOCTOR OF PHILOSOPHY \\ in Physics \\ by \\ Glenn DelFosse Blanford Jr.
}

\author{
Dissertation Comittee: \\ Professor Jonas Schultz, co-Chair \\ Professor Mark A. Mandelkern, co-Chair \\ Professor Dennis Silverman
}


(c) 1997 by Glenn DelFosse Blanford, Jr.

All rights reserved. 
The dissertation of Glenn DelFosse Blanford, Jr. is approved, and is acceptable in quality and form for publication on microfilm:

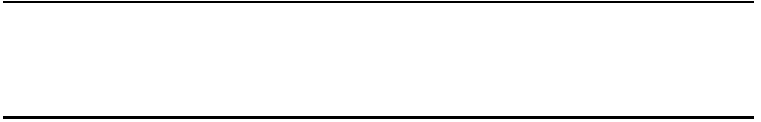

Committee Chair

University of California, Irvine 
To

my father and mother,

Glenn and Florence

Blanford 


\section{Contents}

List of Figures $\ldots \ldots \ldots \ldots \ldots \ldots \ldots \ldots \ldots \ldots$ vii

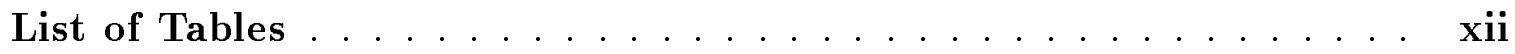

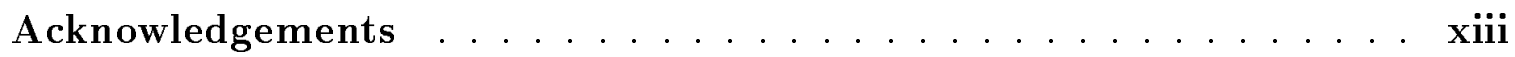

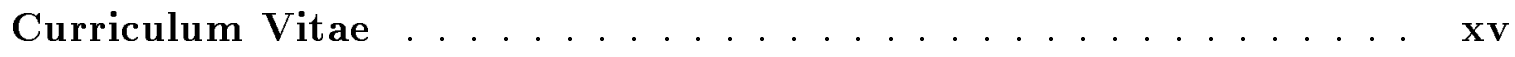

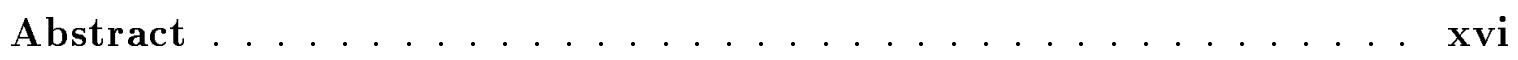

Chapter 1 Theoretical Motivation ............. 1

1.1 Introduction . . . . . . . . . . . . . . . 1

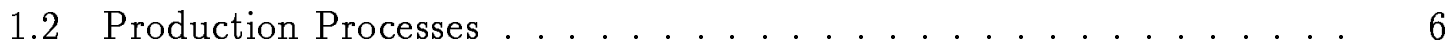

1.2.1 Radiative Recombination . . . . . . . . . . . . 8

1.2.2 Laser Stimulated Radiative Capture . . . . . . . . . . . 9 9

1.2.3 The 3-body Process, $\bar{p} e^{+} e^{+} \rightarrow \bar{H} e^{+} \ldots \ldots \ldots \ldots \ldots$

1.2 .4 Using Positronium . . . . . . . . . . . . . . 10

1.2 .5 Antiprotonic Helium . . . . . . . . . . . . . 10

1.3 Production at Relativistic Energies . . . . . . . . . . . . . 11

1.4 The CERN PS210 Experiment at LEAR . . . . . . . . . . . 21

Chapter 2 Antiproton Beam and Target ............ 24

2.1 Antiproton Accumulator . . . . . . . . . . . . . . . . . 24

2.1.1 Antiproton Production and Cooling . . . . . . . . . . 24

2.1.2 Deceleration Procedure . . . . . . . . . . . . . . 27

2.1.3 Energy Measurement . . . . . . . . . . . . . . . . 29

2.2 Gas Jet Target . . . . . . . . . . . . . . . . . 32

2.3 Charmonium Program . . . . . . . . . . . . . . 37

2.4 Luminosity . . . . . . . . . . . . . . . . . 39

Chapter 3 The E862 Positron Spectrometer. . . . . . . . . 42

$3.1 \quad$ E862 Detectors . . . . . . . . . . . . . . . . . 42

3.2 Foil Stripper . . . . . . . . . . . . . . . . . . 45

3.3 Radioactive Sources . . . . . . . . . . . . . . . 48

3.4 Antihydrogen Beam Parameters . . . . . . . . . . . . . 49

3.5 Positron Magnets . . . . . . . . . . . . . . . . . . 52

3.5.1 Design and Simulations. . . . . . . . . . . . . 52

3.5.2 Construction of Magnets . . . . . . . . . . . . 57 
3.5.3 Magnetic Field Properties . . . . . . . . . . . . . 58

3.5.4 Beam Test Apparatus . . . . . . . . . . . . . . 59

3.5.5 Monoenergetic Electron Source Testing . . . . . . . . . . . 60

3.6 Positron Detectors . . . . . . . . . . . . . . . . . 67

3.6.1 Positron Counter . . . . . . . . . . . . . . . 68

3.6.2 Sodium Iodide(T1) Gamma Ray Detector . . . . . . . . . 70

3.6.3 Positron Source Testing . . . . . . . . . . . . . . . . 73

3.7 Veto Counters and Shielding . . . . . . . . . . . . . . . . 81

3.8 Aperture Changes . . . . . . . . . . . . . . . . . . . 82

Chapter 4 The E862 Antiproton Spectrometer ........ 86

4.1 Time of Flight . . . . . . . . . . . . . . . 86

4.2 Antiproton momentum measurement . . . . . . . . . . 92

4.2.1 Wire Chamber Description . . . . . . . . . . . . . . 92

4.2.2 Momentum Resolution . . . . . . . . . . . . . . . . . 98

4.3 Antiproton Beamline Alignment . . . . . . . . . . . . . . . . . 99

Chapter 5 Trigger and Data Acquisition . . . . . . . . . 103

5.1 Experiment Trigger . . . . . . . . . . . . . . . . . . 103

5.2 Data acquisition . . . . . . . . . . . . . . 106

5.3 Target and Magnet Control . . . . . . . . . . . . . . 107

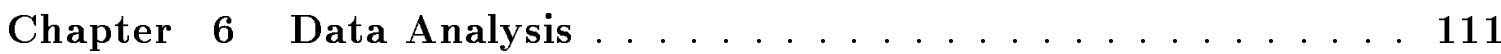

6.1 Introduction . . . . . . . . . . . . . . . . . 111

6.2 Antiproton beam data . . . . . . . . . . . . . . . 112

6.3 Track Reconstruction . . . . . . . . . . . . . . . . . . . 117

6.4 Antiproton Background Tracks . . . . . . . . . . . 125

6.5 Secondary Backgrounds . . . . . . . . . . . . . . 131

6.6 Foil Target-In Data . . . . . . . . . . . . . . . . . . . . . 133

6.6.1 Description of Foil-In Data . . . . . . . . . . . . . 133

6.6.2 Foil-In Momentum Distributions . . . . . . . . . . . . . . 134

6.6 .3 Profiles at foil . . . . . . . . . . . . . . . 137

6.6.4 Emittance Measurements . . . . . . . . . . . . . . . . 140

6.6 .5 Profiles at jet . . . . . . . . . . . . . . . 143

6.6.6 Positron Energies and Annihilation Spectra . . . . . . . . 148

6.6 .7 Timing Analysis . . . . . . . . . . . . . . . . 160

6.7 Foil Target-Out data . . . . . . . . . . . . . . . . . . . . 169

6.7.1 Description of Foil-Out Data . . . . . . . . . . . . . 169

6.7 .2 Jet Location . . . . . . . . . . . . . . . . . . . . . 170

6.7.3 Momentum Distributions . . . . . . . . . . . . . . . . 172

6.7.4 Background to Foil-out Candidates . . . . . . . . . . 175

6.8 Event Selection Summary . . . . . . . . . . . . . . . 178 
Chapter 7 Geometrical Acceptance and Efficiencies . . . . . . 180

7.1 Simulation of foil-out candidates . . . . . . . . . . . . 180

7.2 Simulation of positron beamline . . . . . . . . . . . . 187

7.2 .1 Transport Matrices . . . . . . . . . . . . . . . 188

7.2 .2 Magnet Fields . . . . . . . . . . . . . . . . . . . . 190

7.3 Simulation of antiproton momentum reconstruction . . . . . . . 209

7.4 Efficiencies . . . . . . . . . . . . . . . . . . . 211

7.4.1 Trigger Efficiencies . . . . . . . . . . . . . . . . 211

7.4 .2 Tracking Efficiency . . . . . . . . . . . . . . . 216

7.4 .3 Efficiencies Summary . . . . . . . . . . . . . . . 218

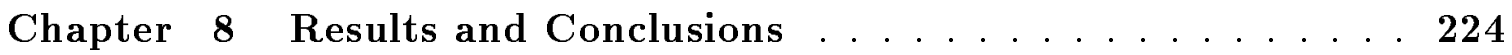

8.1 Observation of Antihydrogen Events . . . . . . . . . . . 224

8.2 Cross Section for $6 \mathrm{GeV} / \mathrm{c} \ldots \ldots \ldots \ldots \ldots \ldots . \ldots \ldots 232$

8.3 Energy Dependence of the Cross Section . . . . . . . . . . 237

8.4 Systematic Errors . . . . . . . . . . . . . . . . . . . . . . 245

8.5 Conclusions . . . . . . . . . . . . . . . . . 247

Bibliography . . . . . . . . . . . . . . . . 249

Appendix A Relativistic Cross Section Calculation . . . . . . 258

A.1 Photoelectric Matrix Element . . . . . . . . . . . . . . 258

A.2 Cross Section Integration . . . . . . . . . . . . . 267

Appendix B Spectroscopy of Relativistic $\bar{H} \ldots \ldots \ldots \ldots$

B.1 Goals . . . . . . . . . . . . . . . . . . 270

B.2 Unperturbed Spectrum . . . . . . . . . . . . . . . 271

B.3 Increasing the Production Rate . . . . . . . . . . . . . . 271

B.4 Effect of Magnetic Fields . . . . . . . . . . . . . . . . . 273

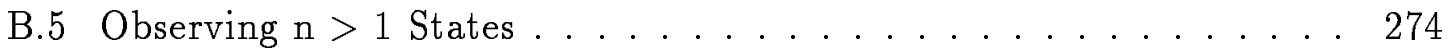

B.6 Measuring a Lamb Shift in antihydrogen . . . . . . . . . 275 


\section{List of Figures}

1.1 Phasor diagram showing $\mathrm{CP}$ and CPT violating parameters. . . . . 3

1.2 Antihydrogen by pair production and $e^{+}$capture. . . . . . . . . 13

1.3 Predicted antihydrogen production cross section vs. momentum. . . 15

1.4 Energy distribution of electrons . . . . . . . . . . . . 16

1.5 Theoretical cross section calculations in the range of energies available to E862. .......................... 19

1.6 Theoretical cross section calculations in a much higher range of energies. 20

1.7 Diagram of PS 210 apparatus [5]. . . . . . . . . . . 23

2.1 Placement of E862 equipment in the tunnel, to scale. . . . . . . 25

2.2 Antiproton Source rings . . . . . . . . . . . . . 28

2.3 Density during stacking vs. orbit . . . . . . . . . . . . . . . . . . 29

2.4 Typical frequency histogram of the beam . . . . . . . . . . . . 31

2.5 Hydrogen jet transverse density profile . . . . . . . . . . . . 33

2.6 Thermodynamic phase space of the hydrogen jet's molecules. . . . . . 34

2.7 Jet-beam intersection . . . . . . . . . . . . . . 35

2.8 Jet target cluster formation, pumps, and chamber dimensions . . . 36

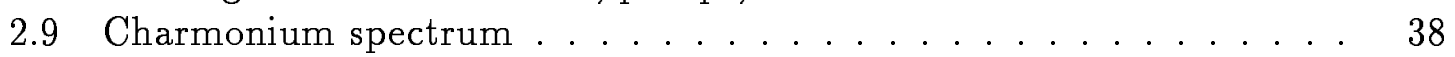

2.10 E835 luminosity monitor angle definition . . . . . . . . . . . . 40

2.11 E835 luminosity monitor spectrum . . . . . . . . . . . . . 40

2.12 Expected production cross section and integrated luminosity . . . . . 41

3.1 Schematic of E862 beamline elements. . . . . . . . . . . . . . . 43

3.2 Cross section for stripping $H^{0}\left(\mathrm{~cm}^{2}\right) \ldots \ldots \ldots$. . . . . . . 46

3.3 Efficiency for stripping $H^{0}$ as a function of carbon foil thickness . . . 47

3.4 Vacuum box for target wheel. . . . . . . . . . . . . . . . 48

3.5 Flying wire profiles of antiproton beam . . . . . . . . . . . 52

3.6 Schematic of E862 beamline elements. . . . . . . . . . . . . 53

$3.740^{\circ}$ dipole for positrons showing flanged edges at $10^{\circ} \ldots \ldots . \ldots 54$

3.8 Diagram of entrance/exit angles for $40^{\circ}$ bend dipole. . . . . . . . . 55

3.9 Focusing solenoid diagram . . . . . . . . . . . . . 55

3.10 Positron tracks (simulated with Transport) at nominal magnetic field. 57

3.11 Positron tracks (simulated) with first solenoid turned off . . . . . . 57

$3.12 \mathrm{e}^{+}$magnetic field nonlinearity . . . . . . . . . . . . . 58

3.13 Deionized water system in Lab 6. . . . . . . . . . . . 61

3.14 Measured magnetic field of solenoid along axis. . . . . . . . . . . . . 62

3.15 Dipole current scans $\mathrm{e}^{+}$spectrometer . . . . . . . . . . . . 63

3.16 Solenoid current scans . . . . . . . . . . . . . . 63 
3.17 Scan over the dipole field for ${ }^{207} \mathrm{Bi}$ after final setup in tunnel. . . . . $\quad 66$

3.18 Scan over the solenoid current for ${ }^{207} \mathrm{Bi}$ after final setup in tunnel. . . 66

3.19 Vertical position scan, for high and low dispersion tunes . . . . . 68

3.20 Probability for an $e^{+}$annihilation in flight . . . . . . . . . . . 71

3.21 Diagram of sodium iodide detector . . . . . . . . . . . . . 73

3.22 High voltage plateaus for the sodium iodide counters. . . . . . . . . 74

3.23 The $\beta^{+}$spectrum of ${ }^{68} \mathrm{Ge} \ldots \ldots \ldots \ldots$. . . . . . . . . . . . . . . . . . . 75

3.24 Gaussian fits to $\mathrm{e}^{+}$and NaI peaks. . . . . . . . . . . . 76

3.25 High dispersion, $\mathrm{e}^{+}$pulse height vs $I_{D} \ldots \ldots \ldots \ldots 77$

3.26 Low dispersion, $\mathrm{e}^{+}$pulse height vs $I_{D} \ldots \ldots \ldots \ldots 77$

3.27 Widths of $\mathrm{e}^{+}$pulse height distributions vs $I_{D} \ldots \ldots \ldots$. . . . . . 78

3.28 Correlation of $\mathrm{e}^{+}$annihilation ray energies in sodium iodide . . . . . 78

$3.29 \mathrm{e}^{+}$pulse height spectrum with ${ }^{68} \mathrm{Ge}$. . . . . . . . . . . . . 79

3.30 Compare gains of $e^{+}$plastic scintillators . . . . . . . . . . . 80

3.31 High dispersion dipole scan. . . . . . . . . . . . . 83

3.32 Low dispersion dipole scan. . . . . . . . . . . . . . . 84

3.33 Vertical ${ }^{207} \mathrm{Bi}$ source position scan. . . . . . . . . . . . . 85

3.34 Positron beam crossing a chord of scintillator. . . . . . . . . . 85

4.1 Voltage divider for time of flight counters. . . . . . . . . . . 88

4.2 Cosmic ray test time of flight counters . . . . . . . . . . . 88

4.3 The effect of time walk on pulse height differences . . . . . . . . . 89

4.4 Time resolution for short, $(30 \mathrm{ft})$ cables . . . . . . . . . . . . . 89

4.5 Time resolution for longer, $(80 \mathrm{ft})$ cables . . . . . . . . . . . 90

4.6 High voltage plateaus for the time of flight counters. . . . . . . . . . 90

4.7 Nonlinearity of one of the fast-TDC channels. . . . . . . . . . . . . 91

4.8 Magnetic field nonlinearity as function of transverse dimension inside an antiproton bend magnet ............... . . 93

4.9 Magnetic field nonlinearity as function of applied current inside an antiproton bend magnet . . . . . . . . . . . . . . . . 93

4.10 Preamplifier schematic for each PWC channel . . . . . . . . . . . 95

4.11 Breakdown showing the various planes of the wire chambers . . . . 97

4.12 Typical transverse and time profiles for test run . . . . . . . . . . 102

5.1 E862 trigger diagram. . . . . . . . . . . . . . 105

5.2 Average rates from the counters as a function of instantaneous luminosity.106

5.3 Data collection and control . . . . . . . . . . . . . . 108

5.4 Target wheel control subsystem. . . . . . . . . . . . 110

6.1 Horizontal angle during the run . . . . . . . . . . . . 113

6.2 Vertical angle during the run . . . . . . . . . . . . . . 113

6.3 Horizontal emittance during the run . . . . . . . . . . . . . . . 114

6.4 Vertical emittance during the run . . . . . . . . . . . . . . 114

6.5 Beam centroid positions at foil vs time . . . . . . . . . . . 115

6.6 Beam centroid positions at foil vs time . . . . . . . . . . . 116

$6.7 \chi^{2}$ distribution from fits to space points . . . . . . . . . 118 
6.8 Number of tracks per event . . . . . . . . . . . . . . . . 119

6.9 Diagram showing how the residual in the bend plane is measured. . . 119

6.10 Vertical track residuals for all one track events . . . . . . . . . . . 120

6.11 Vertical track residuals for triple coincidence events . . . . . . . . . . 120

6.12 Saturation of magnets during deceleration ramp . . . . . . . . . . . . . 122

6.13 PWC profiles of all tracks . . . . . . . . . . . . . 123

6.14 PWC profiles of high momentum, non-candidate events . . . . . . . . 124

6.15 PWC profiles of triple coincidence events. . . . . . . . . . . . . . 124

6.16 Track/beam momentum for foil-in events in runs 1-115 . . . . . . . 126

6.17 Track/beam momentum for foil-in events in runs $1-115$ above transition energy. . . . . . . . . . . . . . . . 126

6.18 Track/beam momentum for foil-in events in runs $1-115$ below transition energy. . . . . . . . . . . . . . . . 127

6.19 Diagram of elastic scattering . . . . . . . . . . . . 128

6.20 Correlation of beam momentum and momentum fraction . . . . . . 129

6.21 Correlation of beam momentum and momentum fraction . . . . . . . 130

6.22 Track momentum/beam momentum for triple coincidence candidates 135

6.23 Track momentum/beam momentum for triple coincidence events, above

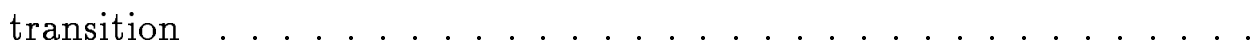

6.24 Track momentum/beam momentum for triple coincidence events, below transition . . . . . . . . . . . . . . 136

6.25 Foil position of all tracks . . . . . . . . . . . . . . 138

6.26 Foil position of triple coincidence events . . . . . . . . . . . 138

6.27 Foil position of high momentum tracks . . . . . . . . . . . . . 139

6.28 Effective size of the antihydrogen beam . . . . . . . . . . . . . 139

6.29 X-Phase space plot of tracks for candidates. . . . . . . . . . . . . 141

6.30 Y-Phase space plot of tracks for candidates. . . . . . . . . . . . . 141

$6.31 \mathrm{X}$-Phase space plot of tracks for all tracks. . . . . . . . . . . . . 142

6.32 Y-Phase space plot of tracks for all tracks. . . . . . . . . . . . . . 142

$6.33 \mathrm{X}$ angular distributions . . . . . . . . . . . . . 144

$6.34 \mathrm{Y}$ angular distributions . . . . . . . . . . . . . . . . 145

6.35 Extrapolated position of track at gas jet for triple coincidence events 146

6.36 Extrapolated position of track at gas jet for all good tracks . . . . . . 146

6.37 Extrapolated position of track at gas jet for triple coincidence events, above transition . . . . . . . . . . . . . . . 147

6.38 Extrapolated position of track at gas jet for triple coincidence events, below transition . . . . . . . . . . . . . . . 147

$6.39 e^{+}$pulse height vs $e^{+}$momentum for candidates . . . . . . . . . . . 149

$6.40 e^{+}$pulse height vs $e^{+}$momentum for candidates . . . . . . . . . . . 149

6.41 Deviation of e+ pulse heights from fit for 1996 triple coincidences . . 150

6.42 Deviation of e+ pulse heights from fit for 1997 triple coincidences . . 150

$6.43 e^{+}$pulse heights for triple coincidence events . . . . . . . . . . . . 152

$6.44 e^{+}$pulse heights for triple coincidence events, corrected for beam momentum dependence . . . . . . . . . . . . . . . 153

6.45 Comparison of $e^{+}$pulse heights for triple coincidences and all events . 154 
6.46 Correlation of $\mathrm{e}^{+}$annihilation ray energies in sodium iodide for calibration data . . . . . . . . . . . . . 156

6.47 Correlation of annihilation $\mathrm{X}$ ray energies in $\mathrm{NaI}$ detector for all events in data.

6.48 Correlation of annihilation $\mathrm{X}$ ray energies in NaI detector for candidate events in data. . . . . . . . . . . . . . . . 157

6.49 Annihilation $\mathrm{X}$ ray energy distributions from the $\mathrm{NaI}$ detector for all events in foil-in data. . . . . . . . . . . . . . . . 158

6.50 Annihilation $\mathrm{X}$ ray energy distributions from the NaI detector for triple coincidence events in foil-in data. . . . . . . . . . . . . 159

6.51 Correlation of TF11 arrival times with horizontal position. . . . . . . 162

6.52 Correlation of TF11 arrival times with ADC TF11 after removing position dependence. . . . . . . . . . . . . . . . . . . 162

6.53 Correlation of TF11 arrival times with ADC TF12 (trigger start PMT) after removing position dependence. . . . . . . . . . . 163

6.54 Correlation of TF21 arrival times with vertical position. . . . . . . 163

6.55 Correlation of TF21 arrival times with ADC TF21. . . . . . . . . . 164

6.56 Timing resolution of TF1 with and without position dependence removed 164

6.57 Timing resolution of $\mathrm{TF} 2 \ldots \ldots \ldots \ldots 5$

6.58 Time of flight distribution using method 1 , normalized to $\beta=1 \ldots 166$

6.59 Time of flight distribution using method 2, normalized to $\beta=1 \ldots 167$

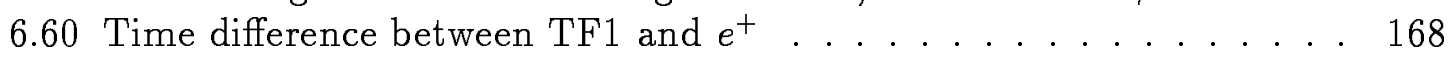

6.61 Transverse position of track at location of jet for foil-out antihydrogen candidates ...................... 170

6.62 Transverse position of track at location of jet for foil-out antihydrogen candidates, above transition . . . . . . . . . . . . 171

6.63 Transverse position of track at location of jet for foil-out antihydrogen candidates, below transition . . . . . . . . . . . . 171

6.64 Reconstructed track/beam momentum for foil-out candidates . . . . . 172

6.65 Reconstructed track/beam momentum for foil-out candidates, above transition ........................ 173

6.66 Reconstructed track/beam momentum for foil-out candidates, below transition ......................... 173

7.1 PWC profiles for $e^{+}$and $\bar{p}-$ set d. . . . . . . . . . . . . . 182

7.2 Steering acceptance as a function of distance from the beam centroid to the center of the foil aperture. . . . . . . . . . . . . . . 184

7.3 Acceptance losses for antihydrogen on apertures . . . . . . . . . 185

7.4 Acceptance $\times$ reconstruction efficiency for missing the positron . . . . 186

7.5 Positron beam profiles at scintillator vs B-field. . . . . . . . . . . . 191

7.6 Simulated optics of a ring image through the positron magnets. . . . 191

7.7 Simulated solenoid longitudinal magnetic field . . . . . . . . . . 193

7.8 Diagram of the sector dipole magnet . . . . . . . . . . . . 194

7.9 Ideal on z-axis magnetic field strength, $B_{y} \ldots \ldots \ldots \ldots 6$

7.10 Ideal tracking using low dispersion, $3 \mathrm{GeV} / \mathrm{c} \bar{p} \ldots \ldots . . . \ldots 198$

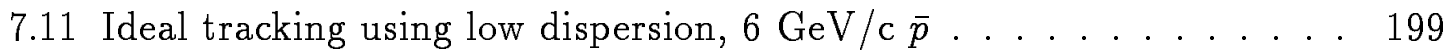


7.12 Ideal tracking using low dispersion, $8.85 \mathrm{GeV} / \mathrm{c} \bar{p} \ldots \ldots . \ldots 200$

7.13 Ideal tracking using high dispersion, $6 \mathrm{GeV} / \mathrm{c} \bar{p} \ldots \ldots . . . . . .201$

7.14 Ideal tracking, low dispersion, $6 \mathrm{GeV} / \mathrm{c} \bar{p}$, with $1 \mathrm{~cm}$ beam offset . . . 202

7.15 Ideal tracking, low dispersion, $6 \mathrm{GeV} / \mathrm{c} \bar{p}$, with $1 \mathrm{~cm}$ beam spread . . 203

7.16 Ideal tracking, low dispersion, $6 \mathrm{GeV} / \mathrm{c} \bar{p}$, with $1 \mathrm{~cm}$ beam offset in $\mathrm{x}$ and $\mathrm{y}$ and $\mathrm{a} 1 \mathrm{~cm}$ beam spread in $\mathrm{x}$ and $\mathrm{y}$. . . . . . . . . 204

7.17 Tracking with magnet offsets . . . . . . . . . . . . . . 205

7.18 Geometrical acceptance of antihydrogens for hitting the foil target . . 207

7.19 Geometrical acceptance for the positron to make it through the $e^{+}$ spectrometer to intercept the scintillator . . . . . . . . . . 208

7.20 Momentum resolution from Monte Carlo. . . . . . . . . . . . . . . 210

7.21 Momentum resolution from data sample containing only candidate events. . . . . . . . . . . . . . 210

7.22 Horizontal position distribution in PWC \#3 . . . . . . . . . . . 212

7.23 Comparison of $\mathrm{TF} 1$ only trigger data. . . . . . . . . . . . . 214

7.24 Ratios of TF1 scalers. . . . . . . . . . . . . . . 215

7.25 Efficiencies for TF2 part of trigger . . . . . . . . . . . . . . 217

7.26 Efficiencies of tracks reconstructed . . . . . . . . . . . . . . . 219

7.27 Total efficiencies for detecting a foil-out type antihydrogen candidate event ...................... 222

7.28 Total efficiencies for detecting a triple coincidence type antihydrogen candidate event .................. 223

8.1 Number of triple coincidences in equal sensitivity bins. . . . . . . . 233

8.2 Number of triple coincidences with good tracks in equal sensitivity bins.234

8.3 Maximum likelihood function, foil-in . . . . . . . . . . . . 237

8.4 Maximum likelihood function, foil-in, above transition . . . . . . . 238

8.5 Maximum likelihood function, foilin, above transition . . . . . . . . 238

8.6 Maximum likelihood function, foil-out . . . . . . . . . . . . 239

8.7 Maximum likelihood function, foil-out, above transition . . . . . . . 239

8.8 Events per luminosity using 10 bins in momentum. . . . . . . . . 240

8.9 Cross section using 10 bins in momentum . . . . . . . . . . . . 241

8.10 Cross section using 25 bins in momentum . . . . . . . . . . . 242

8.11 Antihydrogen production cross section . . . . . . . . . . . 244

A.1 Results of integrating capture cross section from[47]. . . . . . . . . 269

B.1 The hydrogen spectrum in zero field. . . . . . . . . . . . 272

B.2 Hydrogen ionization rates in magnetic fields for $\gamma=9 . \quad \ldots . . . .2274$

B.3 Experimental apparatus needed to measure Lamb shift. . . . . . . . . 277

B.4 Oscillatory probabilities for existing in the $n=2$ antihydrogen states (long,medium,short) within the zero field region as a function of flight distance.................... 279 


\section{List of Tables}

1.1 CPT measurements . . . . . . . . . . . . . . 4

1.2 Hydrogen Lamb shift calculations and measurements. . . . . . . . . 5

1.3 Theoretical rates of antihydrogen formation . . . . . . . . . 11

3.1 Ionization rates and mean decay lengths for antihydrogen atoms ... 44

3.2 Antiproton beam parameters using Fermilab standard emittance . . . 49

3.3 Magnetic field settings vs. beam energy . . . . . . . . . . . . . . 61

3.4 Probability $\mathrm{e}^{+}$will back scatter from the plastic scintillator . . . . . . 69

3.5 Fraction of events with $\mathrm{NaI}$ hits in the data. . . . . . . . . . 79

3.6 Fraction of events in the data with veto counter hits. . . . . . . . 81

4.1 Required resolution to distinguish $K^{-}$and $\pi^{-}$from $\bar{p}$ for equal momenta. 87

4.2 Momentum analysis magnet parameters. . . . . . . . . . . . 92

4.3 Wire chamber wires read out before run $10 \ldots \ldots \ldots \ldots$

4.4 Wire chamber wires read out after run $9 \ldots \ldots \ldots \ldots$

4.5 Multiple scattering contributions . . . . . . . . . . . . 99

4.6 Left and right edges of the beam (inch) . . . . . . . . . . . . 101

5.1 Overall E862 trigger rates for normal running conditions (beam). . . 105

6.1 Interesting events with extra hits . . . . . . . . . 177

6.2 Event selection criteria for antihydrogen candidates. . . . . . . . . 179

6.3 Event selection criteria for antihydrogen candidates. . . . . . . . . 179

7.1 Efficiencies of foil-out events with spectators by Monte Carlo . . . . 183

7.2 Input values to spectrometer simulation. . . . . . . . . . . . 195

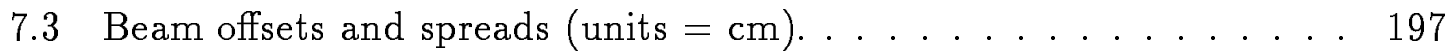

7.4 Magnet offsets (units $=\mathrm{cm}$ ). Acceptance is for $2^{\prime \prime}$ plug. . . . . . . 197

7.5 Wire chamber and tracking efficiencies from three-way coincidences. . 218

7.6 Final corrections to data. . . . . . . . . . . . . . . . 220

8.1 Number of candidate events summary. . . . . . . . . . . 226

8.2 E862 candidate events and integrated luminosity . . . . . . . . . 227

$8.3 \quad$ E862 candidate events and integrated luminosity . . . . . . . . . 228

$8.4 \quad$ E862 candidate events and integrated luminosity . . . . . . . . . 229

8.5 E862 candidate events and integrated luminosity . . . . . . . 230

8.6 E862 candidate events and integrated luminosity . . . . . . . . 231

8.7 Comparison of cross section estimates . . . . . . . . . . 233

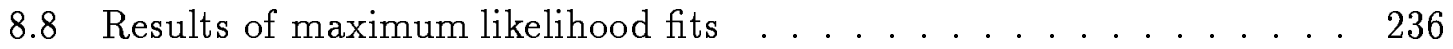

8.9 Cross section results for different foil thicknesses. . . . . . . . . 246 


\section{Acknowledgements}

I would like to thank all the members of the E862 collaboration and of the E835 collaboration for the endless effort and concern about all the issues that make an experiment work especially when two experiments are competing for precious resources. It has been a pleasure working with all of you.

I am grateful to Professors Jonas Schultz and Mark Mandelkern, my dissertation advisors, for their time and efforts in discussions of analyses and preparation of this dissertation. I want to extend special thanks to David Christian and Charles Munger for their constant guidance, imparted knowledge, and enthusiasm.

There are many people connected with the experiment without which we would never have had such success. These include Steve O'Day and the rest of the antiproton group for providing us with such a great beam, the Fermilab-Genoa group, especially M. Macri, for providing and operating the jet target, and the E835 shift crews and luminosity monitor experts. Thanks to the Lab 6 and Lab 7 technicians including $\mathrm{K}$. Mellott, K.C. Cutchlow, H. Schramm, B. Maly, J. Tweed, just to name a few. I also want to thank Jim Westerman for coming to our meetings and offering moral support for what turned out to be a really fun experiment.

I am extremely grateful for the support from my mother and sisters, and my extended family, including Chuck and Pat Blanford, Karen, and Rob Robertie, for

giving me a place to feel at home when I am in California. I also want to thank friends, especially Jose Marques, Greg Griffin, George Zioulas, and Keith Gollwitzer for getting me through some trying times. 
The US Department of Energy under grant DE-FG03-91ER40679 task B has funded this work. 


\section{Curriculum Vitae}

1990 B.S. Joint in Mathematics and Physics, State University of New York at Buffalo

1990 Student Researcher, Los Alamos National Laboratory, SERS Program

1991-1993 Teaching Assistant, Department of Physics, University of California, Irvine

1993-1997 Research Assistant, Department of Physics, University of California, Irvine

1993 M.S. in Physics, University of California, Irvine

1997 Ph.D. in Physics, University of California, Irvine Dissertation: Observation of Relativistic Antihydrogen Atoms

Professor Jonas Schultz, co-Chair Professor Mark Mandelkern, co-Chair

\section{Publications}

T.A. Armstrong et al., Study of the $\eta_{c}$ State of Charmonium Formed in $\bar{p} p$ Annihilations and a Search for the $\eta_{c}^{\prime}$, Phys. Rev.D 52,(9) 1995.

T.A. Armstrong et al., Precision Measurements of Antiproton-Proton Forward Elastic Scattering Parameters in the 3.7 to $6.2 \mathrm{GeV} / \mathrm{c}$ Region, Physics Letters B 385: 479-486, 1996.

T.A. Armstrong et al., Two Body Neutral Final States Produced in AntiprotonProton Annihilations at $2911 \leq \sqrt{(s)} \leq 3686 \mathrm{MeV}$, Phys. Rev. D 56,(5), 1997.

G. Blanford et al., Observation of Atomic Antihydrogen, Phys. Rev. Lett., to be submitted.

G. Blanford et al., Measuring the Antihydrogen Lamb Shift with a Relativistic Antihydrogen Beam, Phys. Rev. D, to be submitted. 


\title{
Abstract of the Dissertation
}

\section{Observation of Relativistic Antihydrogen Atoms}

\author{
by \\ Glenn DelFosse Blanford, Jr. \\ Doctor of Philosophy in Physics \\ University of California, Irvine, 1997 \\ Professor Jonas Schultz, co-Chair Professor Mark Mandelkern, co-Chair
}

An observation of relativistic antihydrogen atoms is reported in this dissertation. Experiment 862 at Fermi National Accelerator Laboratory observed antihydrogen atoms produced by the interaction of a circulating beam of high momentum $(3<$ $\mathrm{p}<9 \mathrm{GeV} / \mathrm{c}$ ) antiprotons and a jet of molecular hydrogen gas. Since the neutral antihydrogen does not bend in the antiproton source magnets, the detectors could be located far from the interaction point on a beamline tangent to the storage ring.

The detection of the antihydrogen is accomplished by ionizing the atoms far from the interaction point. The positron is deflected by a magnetic spectrometer and detected, as are the back to back photons resulting from its annihilation. The antiproton travels a distance long enough for its momentum and time of flight to be measured accurately. 
A statistically significant sample of 101 antihydrogen atoms has been observed. A measurement of the cross section for $\bar{H}^{\circ}$ production is outlined within. The cross section corresponds to the process where a high momentum antiproton causes $e^{+} e^{-}$pair creation near a nucleus with the $e^{+}$being captured by the antiproton. Antihydrogen is the first atom made exclusively of antimatter to be detected. The observation experiment's results are the first step towards an antihydrogen spectroscopy experiment which would measure the $\mathrm{n}=2$ Lamb shift and fine structure. 


\section{Chapter 1}

\section{Theoretical Motivation}

\section{$1.1 \quad$ Introduction}

Although antiparticles have been observed and used in high-energy physics experiments for over 50 years, the anti-matter analogue of the simplest atomic system, hydrogen, had never been observed until 1995. This dissertation describes an experiment which observed antihydrogen atoms confirming a similar observation at CERN (see section 1.4) in 1995 .

Dirac introduced the antimatter concept in the 1932 as a by-product of his relativistic treatment of quantum mechanics [1],[2]. The positron was detected shortly afterwards by C. Anderson in 1932[3]. The antiproton was first observed by O. Chamberlain and E. Segre in 1955 [4]. Antihydrogen, the atomic bound state of the two, waited another forty years to be detected [5].

The verification of the existence of antihydrogen is an exciting discovery. The very tenet that lays the foundation for the existence of antiparticles is the CPT theorem developed by Luders[6], Pauli[7], Bell[8], and Jost[9]. It can be tested in the antihydrogen system. The CPT theorem is the statement that if a process undergoes the combined symmetry operations of time reversal( $\mathrm{T})$, parity or mirror symmetry $(\mathrm{P})$, and charge conjugation (particle - antiparticle symmetry)(C), the process must remain 
unchanged. The CPT theorem relies on the underlying quantum fields being finitedimensional representations of the Lorentz group, that they interact locally, that the spin-statistics theorem is satisfied by the fields and that one can construct a Hermitian Lagrangian from the fields. The CPT symmetry may be violated if the fields do not interact locally (such as in string theories [10] or an extension of quantum mechanics [11]), or if gravity had some CPT-violating effect (for example, Hawking discusses how black holes may violate CPT [12]). These sources indicate any CPT violation would occur on the order of the Planck mass scale, $M_{\text {Planck }}=10^{19} \mathrm{GeV}$.

Although it is difficult to conclude the source of a CPT violation effect, simple searches can be made by doing "null" measurements. These measure the difference in the value of fundamental properties such as mass, lifetime, charge, magnetic moment, etc. between particle and antiparticle. The neutral kaon system mass difference is the best indirect measurement[13]:

$$
\frac{M_{\overline{K_{o}}}-M_{K_{o}}}{M_{K_{o}}} \lesssim 9 \times 10^{-19} \sim \frac{M_{K_{o}}}{M_{\text {Planck }}}
$$

A CPT violation is manifested, similar to $\mathrm{CP}$ violation, in a term in the mass eigenstates.

$$
\begin{aligned}
& K_{S}=K_{1}+(\epsilon+\Delta) K_{2} \\
& K_{L}=K_{2}+(\epsilon-\Delta) K_{1}
\end{aligned}
$$

where $K_{1}, K_{2}$ are $\mathrm{CP}, \mathrm{CPT}$ eigenstates. The indirect $\mathrm{CP}$ violating term $\epsilon$ is $\mathrm{CPT}$ conserving while $\Delta$ is $\mathrm{CP}$ and $\mathrm{CPT}$ violating. The $\Delta$ parameter is actually the phase difference between $\epsilon$ and the ratio of amplitudes for decaying to two pions, $\eta_{+_{-}}$:

$$
\eta_{+-}=\frac{A m p l\left(K_{L} \rightarrow \pi^{+} \pi^{-}\right)}{A m p l\left(K_{S} \rightarrow \pi^{+} \pi^{-}\right)}
$$




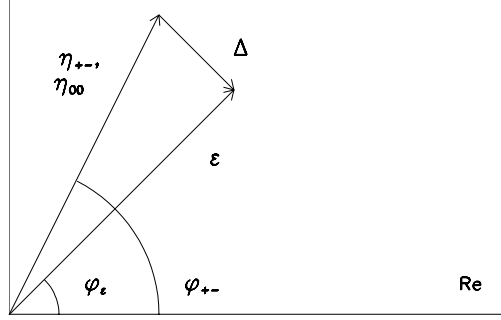

Figure 1.1: Phasor diagram showing $\mathrm{CP}$ and $\mathrm{CPT}$ violating parameters.

The direct CP violation parameter, $\epsilon^{\prime}$ is expected to be collinear with $\epsilon$ but is, at most, $10^{-4}$ times smaller and is ignored here. The phases of $\eta_{+-}$and $\epsilon$ are $\phi_{+-}$and $\phi_{\epsilon}$ respectively. Determining $\epsilon$ involves measurements of $\eta_{+-}$as well as $\eta_{00}$ [14]:

$$
\begin{gathered}
\eta_{00}=\frac{\operatorname{Ampl}\left(K_{L} \rightarrow \pi^{o} \pi^{o}\right)}{\operatorname{Ampl}\left(K_{S} \rightarrow \pi^{o} \pi^{o}\right)} \\
\epsilon=\Delta-\left(2 \eta_{+-}+\eta_{00}\right) / 3
\end{gathered}
$$

The parameter $\Delta$ and thus the neutral kaon mass difference can be found by measurements of $\epsilon, \eta_{+-}$, and $\eta_{00}[15]$.

$$
\begin{gathered}
|\Delta|=\left|\eta_{+-}\right|\left(\phi_{+-}-\phi_{\epsilon}\right) \\
\frac{M_{\overline{K_{o}}}-M_{K_{0}}}{M_{K_{\circ}}}=\frac{\left(M_{L}-M_{S}\right)}{M_{K_{o}}} \frac{2 \Delta}{\sin \phi_{S W}}
\end{gathered}
$$

where $\tan \phi_{S W}=2\left(M_{L}-M_{S}\right) /\left(,_{S}-,_{L}\right)$ is the superweak angle. The $K_{o}-\bar{K}_{o}$ mass difference can be determined from the differences of masses and lifetimes of the observable $\mathrm{K}$ neutral mesons and their decay parameters.

Other null experiments are listed in table 1.1. Many more are listed in [13]. Whereas measurements like the neutral kaon mass difference and the cyclotron frequency measurement (proportional to charge to mass ratio, $\mathrm{q} / \mathrm{m}$ ) can be very precise, 
Table 1.1: CPT measurements

\begin{tabular}{|c||c|c|}
\hline Type & Value & Reference \\
\hline$\left|m_{e^{+}}-m_{e^{-}}\right| / m_{\text {average }}$ & $<4 \times 10^{-8}$ & {$[13]$} \\
$\left|m_{\bar{p}}-m_{p}\right| / m_{p}$ & $<4 \times 10^{-8}$ & {$[16]$} \\
$\left|m_{K^{+}}-m_{K^{-}}\right| / m_{\text {average }}$ & $<(-0.6 \pm 1.8) \times 10^{-4}$ & {$[13]$} \\
$\left|m_{n}-m_{\bar{n}}\right| / m_{\text {average }}$ & $<(9 \pm 5) \times 10^{-5}$ & {$[13]$} \\
$\left|q_{e^{+}}+q_{e^{-}}\right| / q_{e^{-}}$ & $<4 \times 10^{-8}$ & {$[16]$} \\
$\left|q_{p}+q_{\bar{p}}\right| / q_{p}$ & $<2 \times 10^{-5}$ & {$[16]$} \\
$\left|q / m_{e^{+}}+q / m_{e^{-}}\right| /(q / m)_{a v g}$ & $3 \times 10^{-8}$ & {$[16]$} \\
$\left|q / m_{\bar{p}}+q / m_{p}\right| /(q / m)_{\text {avg }}$ & $\sim 10^{-9}$ & \\
$\left|g_{e^{+}}-g_{e^{-}}\right| / g_{e^{-}}$ & $(-0.5 \pm 2.1) \times 10^{-12}$ & {$[13]$} \\
\hline
\end{tabular}

they are not direct measurements of fundamental parameters such as the protonantiproton mass difference.

The hydrogen energy levels are known experimentally to high precision and agree well with quantum electrodynamics calculations. The Lamb shift in hydrogen has been viewed as an excellent place to test CPT invariance. It is composed of different types (order of $\alpha$ ) of corrections to the binding energy. The binding energy without the Lamb shift depends on principal quantum number $\mathrm{n}$ and total angular momentum j [17].

$$
E_{n j}=m_{e} c^{2} f(n, j), f(n, j)=1 / \sqrt{\left[1+\frac{(Z \alpha)^{2}}{(n-\beta)^{2}}\right]}
$$

where $\beta$ is:

$$
\beta=j+\frac{1}{2}-\sqrt{(j+1 / 2)^{2}-(Z \alpha)^{2}}
$$

When $f(n, j)$ is expanded in powers of $Z \alpha$, the nuclear recoil correction is used to modify the result. The nuclear recoil term plus the one-photon radiative corrections to the electron and the one loop corrections to the propagator (vacuum polarization) constitute the main contribution to the Lamb shift which is proportional to $m_{e} e_{e}^{2} e_{p}^{8}$ $[17]$.

$$
E_{n j}=\left(m_{e}+m_{N}\right) c^{2}+m_{r} c^{2}(f-1)+\text { "Lamb shift" }
$$


Table 1.2: Hydrogen Lamb shift calculations and measurements.

\begin{tabular}{|c||c|c|}
\hline Splitting & Value & Reference \\
\hline \multicolumn{3}{|c|}{ Theory } \\
\hline $2 S_{1 / 2}-2 P_{1 / 2}$ & $1057.57(8) \mathrm{MHz}$ & {$[18]$} \\
$2 S_{1 / 2}-2 P_{1 / 2}$ & $1057.912(11) \mathrm{MHz}$ & {$[19]$} \\
$2 S_{1 / 2}-2 P_{1 / 2}$ & $1057.864(14) \mathrm{MHz}$ & {$[20]$} \\
$2 S_{1 / 2}-2 P_{1 / 2}$ & $1057.8576(21) \mathrm{MHz}$ & {$[21]$} \\
\hline \multicolumn{3}{|c|}{ Experiment } \\
\hline $2 S_{1 / 2}-2 P_{1 / 2}$ & $1057.90(6) \mathrm{MHz}$ & {$[22]$} \\
$2 S_{1 / 2}-2 P_{1 / 2}$ & $1057.845(9) \mathrm{MHz}$ & {$[23]$} \\
$2 S_{1 / 2}-2 P_{1 / 2}$ & $1057.893(20) \mathrm{MHz}$ & {$[24]$} \\
$2 S_{1 / 2}-2 P_{1 / 2}$ & $1057.851(2) \mathrm{MHz}$ & {$[25]$} \\
$2 S_{1 / 2}-2 P_{1 / 2}$ & $1057.8594(19) \mathrm{MHz}$ & {$[26]$} \\
$2 S_{1 / 2}-2 P_{1 / 2}$ & $1057.862(20) \mathrm{MHz}$ & {$[27]$} \\
\multicolumn{3}{|c|}{ Inferred } \\
\hline $2 S_{1 / 2}-2 P_{1 / 2}$ & $1057.839(12) \mathrm{MHz}$ & {$[28]$} \\
\hline
\end{tabular}

In the derivation of the hydrogen atom spectrum using the Dirac theory to get equation 1.9 , the $2 P_{1 / 2}$ and $2 S_{1 / 2}$ levels are degenerate. Only when the higher order terms of the recoil correction and Lamb shift are added to the Hamiltonian does one get a splitting. The value of the $2 P_{1 / 2}-2 S_{1 / 2}$ splitting is $1057 \mathrm{MHz}$ or $4.4 \mu \mathrm{eV}[17]$. Comparing the $2 P_{1 / 2}-2 S_{1 / 2}$ Lamb shift splitting in hydrogen and antihydrogen would probe the combined fundamental values which constitute the lowest order term in the Lamb shift The limits in table 1.1, though, indicate the contribution from differences in fundamental constants must be $>8 \times 10^{-5}$ of the splitting. A CPT-violating interaction might still give rise to an appreciable contribution.

$$
\frac{m_{e^{+}} e_{e^{+}}^{2} e_{\bar{p}}^{8}-m_{e^{-}} e_{e^{-}}^{2} e_{p}^{8}}{\text { Average }}
$$

A CPT violation from the electromagnetic interaction of $e^{+}$and $\bar{p}$ may exist even if there is none found in the neutral kaon system . A CPT violating interaction at the level of $10^{-6}$ of the antihydrogen binding energy could show up in a $10 \%$ measurement of the Lamb shift splitting. Appendix 2 outlines an experimental method 
to measure the Lamb shift splitting and fine structure splitting in antihydrogen in order to compare with the precisely known energy splittings of hydrogen.

In addition, the effect of gravity on charged antiparticles has not been observed because the strength of the gravitational force is much smaller than the electromagnetic force on a typical charged antiparticle such as an antiproton. Neutral antihydrogen could eventually be used to test whether antiparticles are attracted to matter particles. Even so, stray electromagnetic fields would need to be very well shielded because antihydrogen will interact with a magnetic field via its magnetic moment. It is reasonable to think anti-particles are gravitationally attracted to each other but the gravitational force between matter and anti-matter is not known experimentally. Experiments are conceivable with cold samples of antihydrogen. For example, the change in gravitational potential energy of antihydrogen is only $10^{-7} \mathrm{eV}$ over $1 \mathrm{me}-$ ter. A highly relativistic beam such as that used in E862 will not be suitable for a gravity experiment since the precision needed for a gravity measurement is much too great. In addition, the baselines available in the beam tunnel environment were not long enough to consider a rough gravitational measurement.

\section{$1.2 \quad$ Production Processes}

The following sections from the scientific literature describe various possible antihydrogen production methods that are being actively researched. All these methods, including the relativistic pair production with $e^{+}$capture, rely on the ability to produce sufficient quantities of antiprotons and positrons and on getting them to interact at low relative velocity in order to bind. Antiprotons can presently be created only at Fermilab's antiproton source and at CERN's antiproton source. Only E862 and its successor spectroscopy experiment (described below) have produced or plan 
to produce and observe antihydrogen at Fermilab. Two experiments have been proposed at CERN's new antiproton decelerator, ATRAP[29] and ATHENA[30]. Both propose to bring clouds of antiprotons and positrons together at low relative velocity within nested Penning or combined Penning-Paul traps.

The antiproton production method used at CERN is not fundamentally different than that used at Fermilab (see section 2.1.1). Antiprotons are accumulated in the accumulator and decelerated in the antiproton decelerator (AD). Five years ago, they would have been transferred to the Low Energy Antiproton storage Ring (LEAR) for further deceleration, cooling, and storage. Since the beam (kinetic) energy of LEAR antiprotons could be reduced to less than $5 \mathrm{MeV}$ [35], antiprotons could be further reduced in energy by use of moderating material which is usually a thin foil. This introduces an energy spread, and the efficiency for collecting low energy antiprotons is small, but antiprotons at the final energies remaining after moderation $(\sim 10 \mathrm{keV})$ can be injected into a charged particle trap. The LEAR antiproton beam was delivered in either pulsed (50-400 nsec spills) or near-continuous (up to 1 hour spills). Positrons are produced from a radioactive source $\left(\beta^{+}\right.$spectrum, usually $\left.{ }^{22} \mathrm{Na}\right)$. They are slowed by subsequent moderation in solid neon or a buffer gas.

A Penning trap[36] is a set of electrodes and permanent magnets arranged so that there is a constant, axial magnetic field along the symmetry axis as well as an electric quadrupole field in the transverse plane. The resulting motion of a charged particle in a Penning trap can be expressed as a sum of three motions: a slow $E \times B$ drift around the symmetry axis; a medium axial oscillation along that axis; and a fast, small radius cyclotron motion. These traps have been used [37] to separately hold positrons and antiprotons. Combining the two species in a small volume so that they will interact is not trivial because of their mass and charge difference although interactions of protons and electrons in a combined trap have been observed [29]. The 
combined Penning-Paul trap has been used where the Paul trap confines one species of particle within the trough of a radio frequency wave. Nested Penning traps (one trap for each species) have also been used.

Each production method has some bearing on the ability to perform spectroscopic studies on the anti-atoms. Although a method to perform spectroscopy on relativistic antihydrogen is outlined in appendix B.6, it is fundamentally different from what is envisioned for low temperature antihydrogen. The method involves an interference of the $\mathrm{n}=2$ antihydrogen atomic states after a large Stark electric field mixes the states. The long-lived state is allowed to propagate in zero field whereupon vacuum regeneration of other states takes place and can be measured. The distances are such that the fine structure and Lamb shift splittings can be measured. The low temperature experiments envisage the cooling of the anti-atoms within a magnetic field configuration so that high-precision spectroscopic studies could be performed. The topics discussed in this section can be found in greater detail in [35].

\subsubsection{Radiative Recombination}

The simplest method is spontaneous recombination of the antiproton and positron:

$$
\bar{p}+e^{+} \rightarrow \bar{H}+\gamma
$$

The cross section depends on the ratio of ionization energy to energy (or velocity) difference between the $\bar{p}$ and $e^{+}$.

$$
\sigma=\left(2.1 \times 10^{-22} c m^{2}\right) \frac{E_{0}^{2}}{n E_{e}\left(E_{0}+n^{2} E_{e}\right)}
$$

where $E_{e}$ is the kinetic energy of the $e^{+}$and $E_{0}=13.6 \mathrm{eV}[38],[39]$. The $1 / n^{3}$ [40] dependence on principal quantum number favors ground state production. Radiative recombination of electrons and protons into hydrogen atoms has been observed in a 
storage ring [41]. For the optimal scenario at the CERN AD of $n_{e^{+}}=10^{8} \mathrm{~cm}^{-3}$ and $N_{\bar{p}}=10^{6}$, a rate of $10^{3} \mathrm{sec}^{-1}$ is expected.

\subsubsection{Laser Stimulated Radiative Capture}

The rate is small but can be enhanced by stimulated capture. One method entails separate beams of positrons and antiprotons which are merged with small relative velocity. A high power laser can be used to stimulate transitions from either the continuum or Rydberg states to the lower levels.

$$
\bar{p}+e^{+}+N \gamma \rightarrow \bar{H}+(N+1) \gamma
$$

where $\mathrm{N}$ is the number of extra photons contributed by the laser. To merge beams of $\bar{p}$ and $e^{+}$, a storage ring accommodating both species would have to be built. Stimulated recombination of electrons and protons into hydrogen atoms has been observed in a storage ring where each species had $\beta=0.0265$ [42],[38]. Laser stimulated capture can also be used with intersecting plasmas. For $N_{\bar{p}}=10^{6}$ antiprotons and $n_{e^{+}}=10^{8} \mathrm{~cm}^{-3}$ positrons, a much larger rate compared to spontaneous recombination of $10^{5} \mathrm{sec}^{-1}$ for de-excitation into the ground state and even $10^{8} \mathrm{sec}^{-1}$ for de-excitation into $n$ $=10$ can be achieved. The cross section is enhanced over equation 1.14 by a factor $\frac{P c^{2}}{F \Delta \nu \pi h \nu^{3}}$ where $P$ is the laser power in watts, $F$ is the cross sectional area of the laser beam, and $\nu$ is the laser frequency [38]. Transfer of positrons from the continuum to the Rydberg levels can be accomplished separately by 3-body collisions or stray fields. Lasers can stimulate transitions from these states to the $2 \mathrm{P}$ state where spontaneous decay to the ground state will occur at high rate $\left(R_{2 P \rightarrow 1 S}=6.3 \times 10^{-8} \mathrm{~ns}\right)[43]$. 


\subsubsection{The 3-body Process, $\bar{p} e^{+} e^{+} \rightarrow \bar{H} e^{+}$}

Three-body collisions $\bar{p} e^{+} e^{+} \rightarrow \bar{H} e^{+}$would cause the antiproton and positron to recombine into $(n \sim 100)$ Rydberg levels of antihydrogen. The anti-atom would be collisionally de-excited down to $n \sim 40$ where radiative transitions become more favorable. The rate can be quite large compared to the two body processes but the large principal quantum number states can be reionized easily. The three-body process with an electron is conceivable $\bar{p} e^{+} e^{-} \rightarrow \bar{H} e^{-}$but competes against $e^{+} e^{-}$ annihilation.

\subsubsection{Using Positronium}

Exchanging a positron between positronium and an antiproton is enhanced when the Ps is in an excited state $(n>10)[44]$. One scheme would form Ps, then two lasers excite the $(1 S-2 P)$ and $(2 P-n l)$ transitions. Afterwards they would enter a Penning trap where the excited positronium collide with cold $(\mathrm{T}=4 \mathrm{~K}) \vec{p}$ s. The antihydrogen must still be de-excited in a radiative transition with a laser since the higher-n states are less stable. The antiprotons can be accumulated in the trap ahead of time, so the rate of recombination depends directly on the achievable positronium flux.

\subsubsection{Antiprotonic Helium}

Antiprotonic helium $\left(\bar{p}(H e)^{+}\right)$is a metastable exotic atom which has been formed with a $3 \%$ efficiency at KEK (Japan) [45],[46] when $\vec{p}$ s pass through Helium (liquid or solid)[45]. The atom is in a linear combination of states with high $(n<30)$ 
Table 1.3: Theoretical rates of antihydrogen formation. The first two entries assume $10^{6} \bar{p}$ and $10^{8} e^{+} \mathrm{cm}^{-3}$ and both species at $\mathrm{T}=4 \mathrm{~K}$. The next three entries assume roughly $10^{5} \bar{p}$ /hour. The relativistic production rates assume the energies and particle densities available while the formation experiment took place. The relativistic production at LEAR is for a hydrogen gas jet.

\begin{tabular}{|c||c|}
\hline Method & Recombination Rate $(\bar{H} / \mathrm{sec})$ \\
\hline Radiative & $3 \times 10^{3}$ \\
Laser-Stimulated & $10^{5} \rightarrow 3 \times 10^{8}$ \\
Positronium & $10 \rightarrow 100$ \\
Three-body recombination & 600 \\
$\bar{p}(H e)^{+}$ & $10^{3}-10^{4}$ \\
Relativistic Production (LEAR) & $1.9 \times 10^{-7}$ \\
Relativistic Production (FNAL) & $6 \times 10^{-5}$ \\
\hline
\end{tabular}

$\bar{p}$ principal quantum number states and an electron in a 1S state. The possible production reactions are:

$$
\begin{gathered}
\left(\bar{p}(H e)^{+}\right)_{N L}+e^{+} \rightarrow \bar{p} e^{+}+(H e)^{+}-11 e V \\
\left(\bar{p}(H e)^{+}\right)_{N L}+P s \rightarrow \bar{p} e^{+}+(H e)^{0}+6.8 e V
\end{gathered}
$$

The latter is more likely to produce antihydrogen. The positronium must not be ionized as it travels through the helium though. The $\bar{p}(H e)^{+}$lifetime is long $(3 \mu \mathrm{s})$ compared to the time it takes to bind although one would think the antiproton would annihilate much more rapidly (on the order of psec). The $\bar{p}(H e)^{+}$will decay by an Auger transition involving the antiproton and an emitted electron followed by a subsequent annihilation.

\subsection{Production at Relativistic Energies}

The method Fermilab experiment E862 used was radically different from those above since it relied on a highly relativistic beam of antiprotons which intersected a molecular gas jet target. When an antiproton passes through the Coulomb electric 
field of the target nucleus, it can cause the nucleus to produce an $e^{+} e^{-}$pair through a virtual photon from the nuclear Coulomb field, (see figure 1.2). Some fraction of the positron phase space includes velocities close to the antiproton velocity and the positron may become bound to the $\bar{p}$. Calculations have been performed of the QED process [47],[48],[49] (see Appendix A). At the energy of the Fermilab antiproton source, the cross section for this type of antihydrogen production is a few $\mathrm{pb}$, (see figure 1.3) with hydrogen gas as the target. E862 was originally proposed to collect $200 \mathrm{pb}^{-1}$ of integrated luminosity yielding about 760 atoms of antihydrogen.

The electromagnetic process was initially investigated in connection with a similar QCD process, photoproduction of charmonium bound states within a nucleus. Charm quark pairs can be produced when a hard photon travels into a nucleus. Bound states such as $J / \psi(c \bar{c})$ can be produced but the binding takes place when the quark-antiquark pair are well outside the nucleus, far removed from the location of the pair-production [50],[51],[52]. The equivalent QED process for an atom is interesting because of its relative ease of calculation and complete knowledge of the wave functions. The expected cross section for free pair production [53],

$$
\sigma_{\bar{p} p \rightarrow \bar{p} p e^{+} e^{-}}=\frac{28}{27 \pi}\left(Z_{1} Z_{2} \alpha r_{e}\right)^{2} \cdot \ln ^{3}\left(\frac{2 \gamma^{2}-1}{2}\right)
$$

is about $70 \mu b$ for $\gamma=6$.

Actually two similar processes can lead to antihydrogen production. The two (virtual, spacelike) photon production, $p \bar{p} \rightarrow \gamma^{*} \gamma^{*} p \bar{p} \rightarrow e^{+} e^{-} \bar{p} p \rightarrow \bar{H} e^{-} p$ is of interest to antihydrogen production. This reaction is shown in figure 1.2 and has a cross section on the order of $\mathrm{pb}$ for $\bar{p} \mathrm{GeV}$ energies. The bremsstrahlung reaction, $\bar{p} p \rightarrow$ $\gamma^{*} \bar{p} p \rightarrow e^{+} e^{-} \bar{p} p \rightarrow \bar{H}^{\circ} e^{-} p$, where the virtual photon is timelike, has a cross section on the order of fb for $\bar{p} \mathrm{GeV}$ energies. The cross section is related to the $\bar{p}$ elastic scattering cross section with the assumption that the $e^{+}$has a small momenta relative 


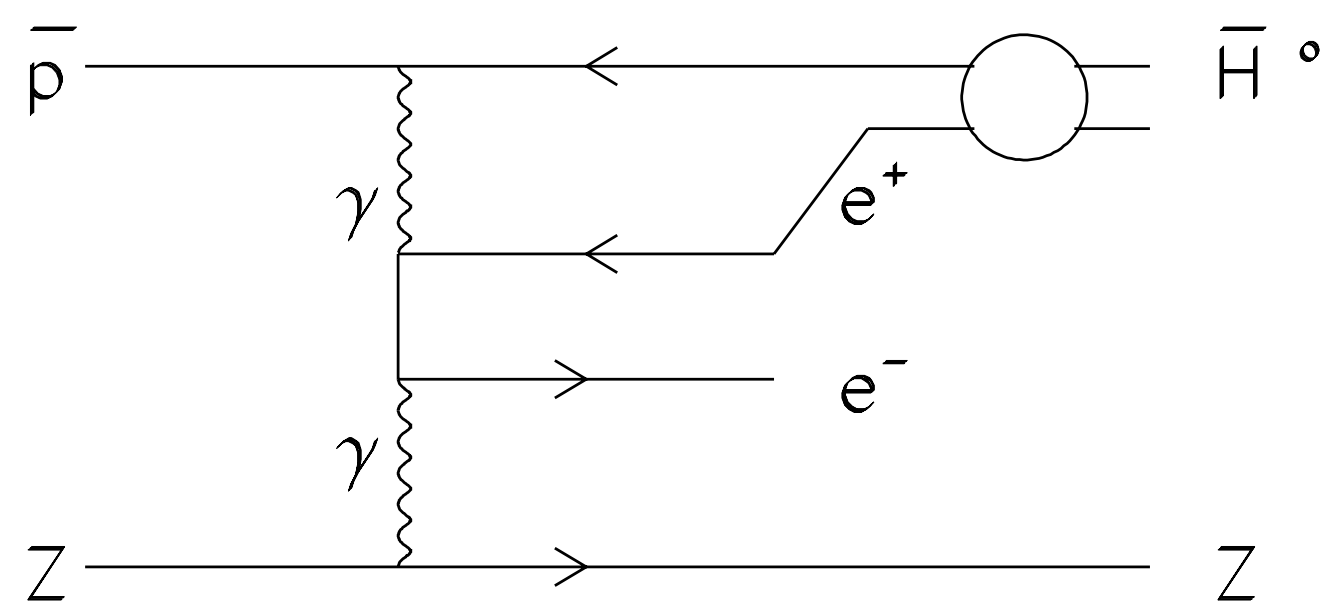

Figure 1.2: Antihydrogen by pair production and $e^{+}$capture.

to the $\bar{p}[48]$,

$$
\sigma_{\text {bremms }}=\frac{4 \alpha^{5}}{3 \gamma^{2} \pi^{2}}\left(\frac{d \sigma}{d \Omega}\right)_{\bar{p}, \text { elastic }}
$$

It is suppressed by $10^{-3}$ relative to the two photon process for $\mathrm{GeV}$ scale projectile energies making it unimportant for antihydrogen production.

The calculation of the cross section for antihydrogen production used in [47] is performed in the rest frame of the antiproton which is considered the same as the rest frame of the antihydrogen. The equivalent photon method relates the process $p \bar{p} \rightarrow p e^{-} \bar{H}$ to a similar process, $\gamma^{*} \bar{p} \rightarrow e^{-} \bar{H}$ where the proton's Coulomb field is basically Fourier analyzed into contributions from virtual photons, $\gamma^{*}$, of different energy and transverse momentum. The virtual photon is considered to be nearly on mass shell ( $q^{2} \sim 0$, where $\mathrm{q}$ is the photon four momentum). The photoelectric process, $\gamma^{*} H \rightarrow e^{-} p$, is related to the capture process $\gamma^{*} \bar{p} \rightarrow e^{-} \bar{H}$. They are related through crossing symmetry. The photoelectric matrix element can be expressed in terms of the Mandelstam variables $(\mathrm{s}, \mathrm{t}, \mathrm{u})$ [54]. The details of how the expression for the matrix element comes about are outlined in the appendix. When $s$ and $t$ are interchanged (crossing symmetry), the result is the matrix element for capture, $M\left(\gamma^{*} \bar{p} \rightarrow e^{-} \bar{H}\right)$. The matrix element, $M\left(\gamma^{*} \bar{H} \rightarrow e^{-} \bar{p}\right)$ already contains the factor 
related to the interaction and kinematics (proportional to $\alpha^{4}$ ) and the factor dealing with capture. The latter involves the $\bar{H}$ wavefunction at $\mathrm{r}=0$ and provides a factor of $\alpha^{3}$ to the cross section so the total cross section is proportional to $\alpha^{7}$.

$$
\left|\Psi_{1 S}(0)\right|^{2}=\frac{1}{4 a_{0}^{3}} \frac{1}{4 \pi}, a_{0}=0.53 \times 10^{-10} \mathrm{~m}[55]
$$

The result after invoking crossing symmetry on the photoelectric hydrogen matrix element is squared and the spins summed to get a cross section, $\left|M_{\text {capture }}(s, t, u)\right|^{2}=$ $2\left|M_{\text {photo }}(t, s, u)\right|^{2}$. This cross section for one photon is summed over all photon four-momenta using the equivalent photon method of virtual quanta [49],[47],[56] $\left(\gamma=E_{l a b, \bar{p}} / M_{\bar{p}}, M_{\bar{p}}\right.$ is the antiproton mass, $\mathrm{Z}=$ target atomic number $)$ :

$$
\sigma_{\gamma^{*} \bar{p} \rightarrow e^{-} \bar{H}(1 S)}(\omega)=\frac{p}{64 \pi^{2} \omega M^{2}} \int|M|_{\text {capture }}^{2} d \Omega_{e}
$$

$$
\frac{Z^{2} \alpha}{\pi} \int_{2 m / M_{\bar{p} \gamma}}^{1} \frac{d x}{x} \int_{0}^{q_{\perp}^{2} m a x} \frac{q_{\perp}^{2} d q_{\perp}^{2}}{\left(q_{\perp}^{2}+x^{2} M_{\bar{p}}^{2}\right)^{2}}\left[\frac{1+(1-x)^{2}}{2}\right] \begin{gathered}
\sigma_{\bar{p} Z \rightarrow \bar{H}(1 s) Z e^{-}}= \\
\sigma_{\gamma^{*} \bar{p} \rightarrow e^{-} \bar{H}(1 s)}\left(\omega, q^{2}\right)
\end{gathered}
$$

where the factor involving the integration over $q_{\perp}^{2}$, the photon's transverse momentum, originates from the equivalent photon approximation. The variable $\mathrm{x}$ is the ratio $\mathrm{x}$ $=\omega /\left(M_{\bar{p}} \gamma\right)$. Since the photon is nearly on mass shell, $q^{2} \sim 0, q_{\perp} \max \sim \omega$, and $\sigma_{\gamma^{*} \bar{p} \rightarrow e^{-} \bar{H}(1 s)}\left(\omega, q^{2}\right) \rightarrow \sigma_{\gamma^{*} \bar{p} \rightarrow e^{-} \bar{H}(1 s)}(\omega)$. The integration over $q_{\perp}^{2}$ easily yields the energy dependence of the cross section:

$$
F(\gamma)=\ln \left(\gamma^{2}+1\right)-\frac{\gamma^{2}}{\gamma^{2}+1}
$$

Integration of the capture matrix element over the electron's energy and solid angle yields:

$$
\sigma_{\bar{p} Z \rightarrow \bar{H}(1 s) Z e^{-}}(\gamma)=Z^{2} F(\gamma) \frac{\alpha}{\pi} \bar{\sigma}
$$




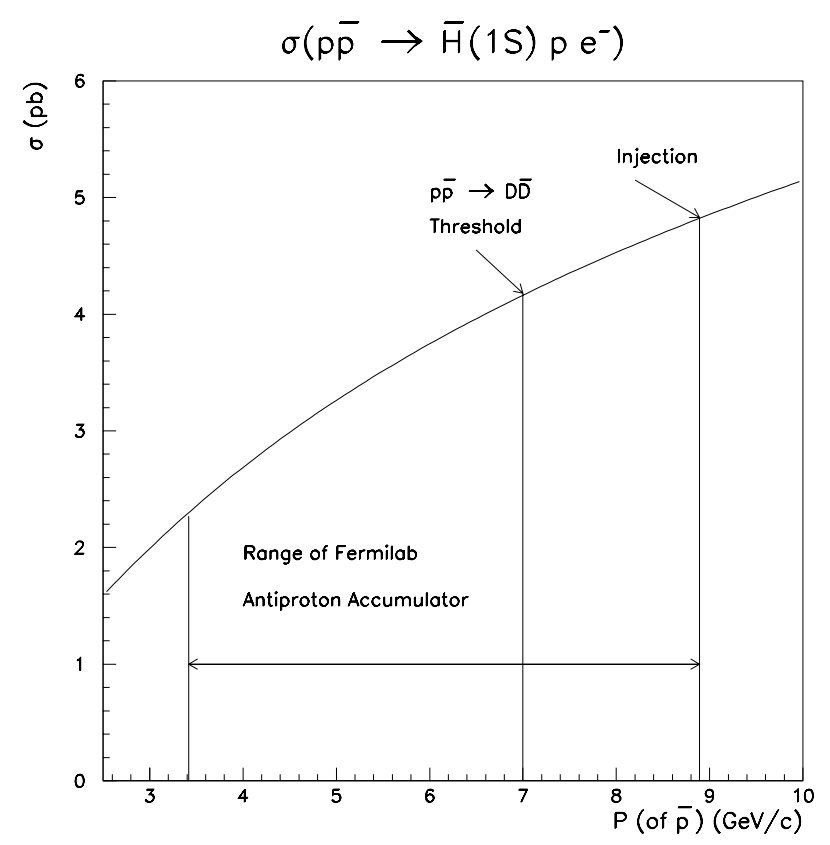

Figure 1.3: Predicted [47] cross section for antihydrogen production vs. $\bar{p}$ lab momentum.

where $\frac{\alpha}{\pi} * \bar{\sigma}=1.426 \mathrm{pb}[47]$.

$$
\frac{\alpha}{\pi} * \bar{\sigma}=\int_{2 m_{e}}^{M_{\bar{p} \gamma}} \frac{d \omega}{\omega} \sigma_{\gamma \bar{p} \rightarrow \bar{H}(1 S) e^{-}}(\omega)
$$

Notice $\sigma_{\bar{H}}$ is dependent logarithmically on energy, (as shown in figure 1.3), but varies as $Z^{2}$, where $Z$ is the atomic number of the target nucleus. The annihilation process $\bar{p} p \rightarrow \bar{D} D$ in figure 1.3 refers to the threshold for this process to occur where $\mathrm{D}$ is the lightest charmed meson.

The cross section for production into excited states is expected to vary as $1 / n^{3}[40]$. Clearly the ground state dominates the production. The production into $n>1$ (primarily S) states contributes a further $20 \%$ to the inclusive production cross section. As described in section 3.1 , only $n=1$ and $n=2$ states contribute to experiment E862's measurement. As is outlined more fully in chapter 9, the excited states are much more sensitive than the ground state to ionization from laboratory magnetic fields. They also can decay through Stark mixing and radiative transitions 


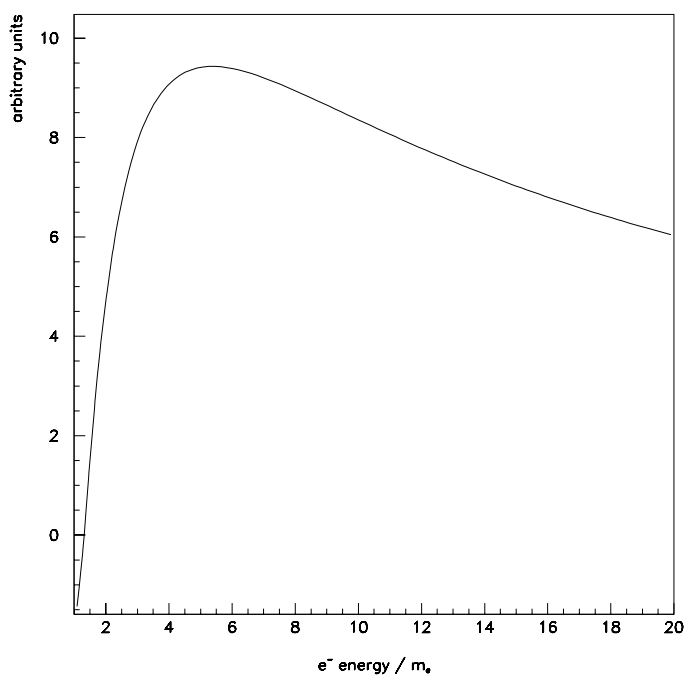

Figure 1.4: Energy distribution of electrons from pair production with K shell capture. Energy is in units of electron mass.

to the ground state in those magnetic fields. Since the antihydrogens pass through the first bend magnet, a beam of pure $1 \mathrm{~S}$ antihydrogen is guaranteed, of which a small fraction arises from excited states decaying to the ground state within the magnet. The momentum transfered to the $\bar{p}$ in the $\bar{H}$ formation is on the order of $m_{e} c$ since the $\bar{p}-e^{+}$relative velocity must be small for the atom to form. The antihydrogen will emerge with nearly the same longitudinal momentum (to within $0.9995 P_{\bar{p}}$ ), and trajectory (to within $10^{-4} \mathrm{rad}$ ) as the antiproton. As pointed out in chapters 3 and 4 , the angular divergence of the beam is at least a couple of mrad, and the measured momentum resolution is about $5 \times 10^{-4}$ at best. Thus the details of the formation process cannot be distinguished using the present apparatus. The electron takes up most of the recoil. In Bertulani and Baur[57], it is shown that, in the antiproton rest frame, the electron energy peaks at about 2 - 3 times the electron mass (see figure 1.4). They are contained in a cone in the lab frame of opening angle about $\Delta \theta \sim 1 / \varepsilon_{e^{-}}$. 
Figures 1.5, and 1.6 portray the theoretical cross sections calculated by various authors. The cross section calculations from [47] and [48] agree approximately in magnitude and as a function of energy. The process, (see figure 1.2), is insensitive to the sign of the charge of the projectile; the antiproton could be replaced by a proton or nucleus of charge $Z_{2}$, in which case, a factor of $Z_{2}^{5}$ arises. This is because when the pair production takes place, if the $e^{+}$or $e^{-}$is to have large enough momentum to be captured by the projectile, it is not strongly affected by the target nucleus electric field. After being produced, since the $e^{+}$and $e^{-}$are charged, the nuclear electric field will either attract or repel the particle which, at low energies, would affect the phase space available for binding to the antiproton. When the projectile or target is a highly charged ion, the nucleus can have a large effect on the outgoing lepton moving the peak energy to larger values because of repulsion. Other calculations have been done [58],[59], [60],[61] for these nucleus - nucleus collisions with pair production and electron capture similar to the antihydrogen calculation. They are important processes to understand they contribute to emittance blowup in relativistic heavy ion colliders such as RHIC[62],[53].

A calculation of a similar process where a transfer of a negative energy $e^{-}$in a continuum state of the target to a positive energy bound state of the projectile has a different energy dependence than the process described previously[63]. The previous process can be considered as an excitation of a negative energy $e^{-}$in a bound state of the projectile into a positive energy bound state of the same projectile. The energy dependence is much different because of momentum constraints. The value of the cross section is similar at higher energies but significantly smaller at the energies of interest to the experiment. Data exists on high energy pair production and capture in near collisions of highly-charged ions at a few $\mathrm{GeV}$ collision energies [64],[65]. 
Recently, new theoretical calculations were published [57], for the antihydrogen production cross section which improve on the results of [47], [48], etc. They yield a smaller cross section which is on the order of $1 \mathrm{pb}$ for momenta near $6 \mathrm{GeV} / \mathrm{c}$. This is further discussed in chapter 8 . 


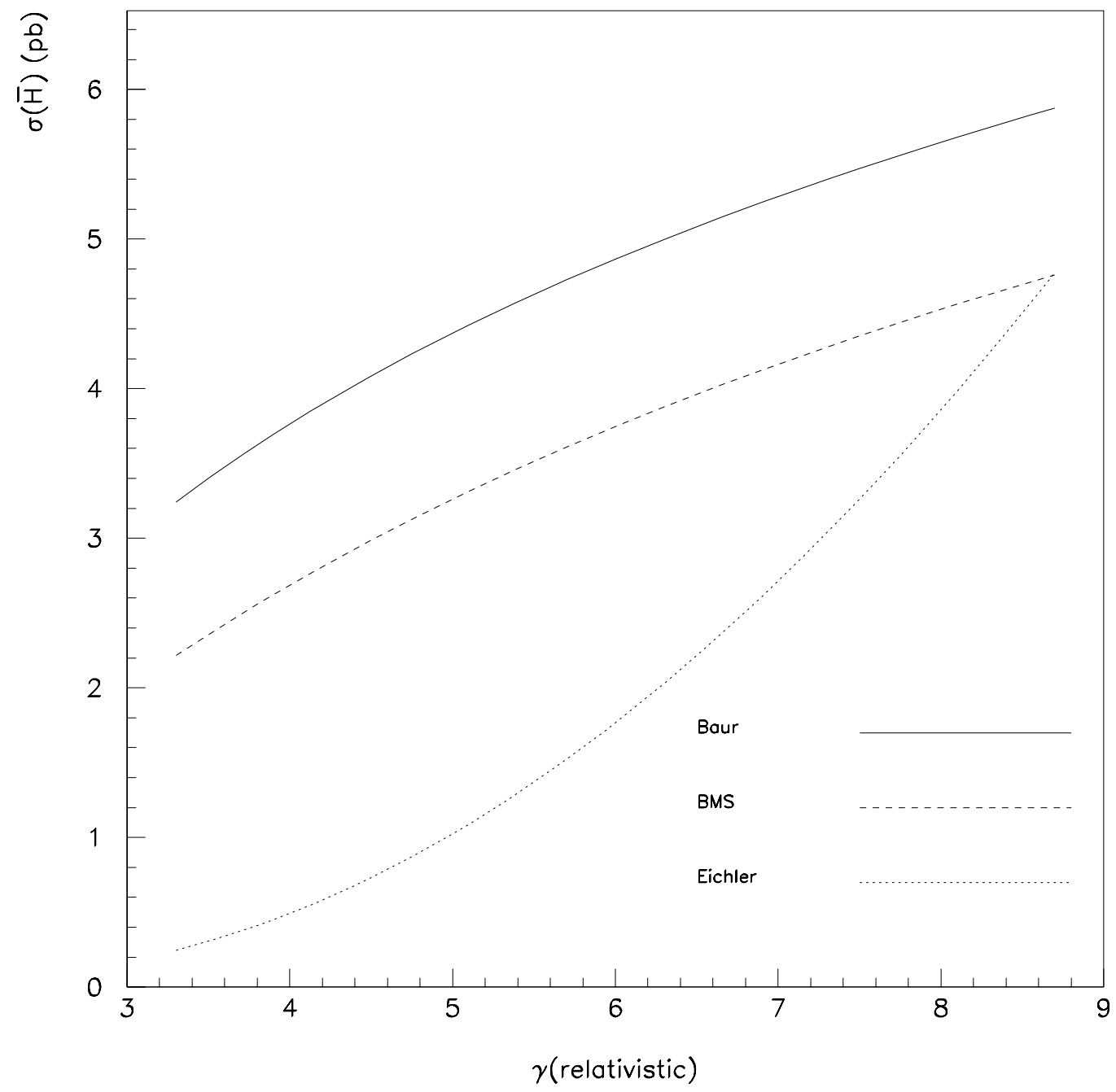

Figure 1.5: Theoretical cross section calculations in the range of energies available to E862. "Baur" refers to [48], "BMS" refers to [47], "Eichler" refers to [63]. 


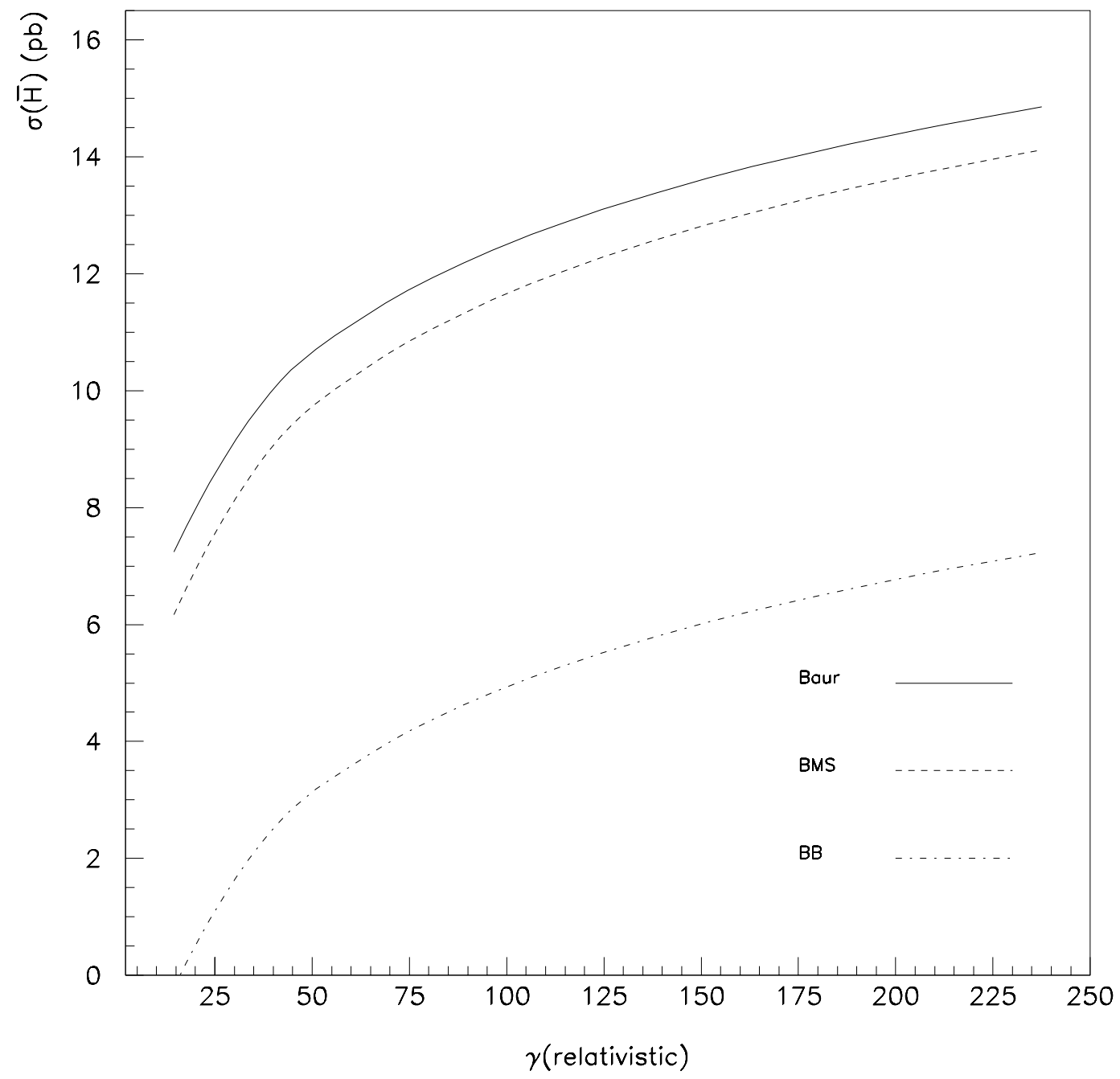

Figure 1.6: Theoretical cross section calculations in a much higher range of energies. "Baur" refers to [48], "BMS" refers to [47], "BB" refers to [40]. 


\subsection{The CERN PS210 Experiment at LEAR}

In September 1995 at CERN, the PS210 collaboration [5] made a claim of discovering 9 antihydrogen atoms at CERN's LEAR (Low Energy Antiproton Ring). Out of approximately $3 \times 10^{5}$ triggers, the group identified 11 antihydrogen candidates. Their background, mostly from the double charge exchange process, $\bar{p} p \rightarrow \bar{n} n \rightarrow \bar{p} p$, was predicted to be $2 \pm 1$ events.

The LEAR antiproton beam momentum $(1.94 \mathrm{GeV} / \mathrm{c})$ used in the antihydrogen experiment is lower than the momenta used at Fermilab. The antihydrogen atoms were formed in a xenon $\left(Z^{2}=2916\right)$ clustered gas jet target [66] whereas E862 used hydrogen. The estimated integrated luminosity was $\int \mathcal{L} d t=5 \mathrm{nb}^{-1}( \pm 50 \%)$ [5]. The large uncertainty comes primarily from poor knowledge of the jet density. The antihydrogens produced in the jet travel down the straight section of the LEAR ring past the interaction point. At the first bend magnet, the antiprotons are bent away. The anti-atoms continue in a straight line until the first silicon detector is encountered where they ionize still within the LEAR vacuum. The ionization products traverse three silicon detectors, $700 \mu \mathrm{m}, 500 \mu \mathrm{m}$ and $700 \mu \mathrm{m}$ thick respectively [5]. Within the first two silicon detectors, the $663 \mathrm{keV}$ kinetic energy positron is stopped and annihilates and a $E_{e^{+}}+(d E / d x)_{\bar{p}}$ measurement is made. The third detector measures only $(d E / d x)_{\bar{p}}$. Surrounding the silicon detectors is a 6-fold segmented $\mathrm{NaI}(\mathrm{Tl})$ cylinder. The gamma rays from the annihilation are detected and an attempt is made to determine whether they are back-to-back. A time of flight measurement of the antiproton $(\beta=0.900)$ is made with 7 scintillation counters and a scintillating fiber hodoscope. The antiproton momentum is measured with three drift chambers $\left(8 \times 8 \mathrm{~cm}^{2}\right)$ and a dipole bend magnet. 
The acceptance of the detectors was estimated to be $\epsilon=0.3$ from Monte Carlo studies and test measurements. Additional losses from antiproton material interactions and wire chamber efficiency (not included in simulations) decreases this by a further $15 \%$ [5]. A large uncertainty was due to imprecise knowledge of the integrated luminosity and a rapidly diverging beam. Event selections were made on the data sample in order to remove mostly single and multi-pion events. The criteria include requiring the energy deposits in the silicon and in the scintillators to agree with expectations and a track in the antiproton spectrometer originating from the interaction. Also required were two $\mathrm{NaI}$ hits in wedges in which each had energies in a band around $511 \mathrm{keV}$ but were not adjacent. No further cuts on the time of flight or momentum were needed. 

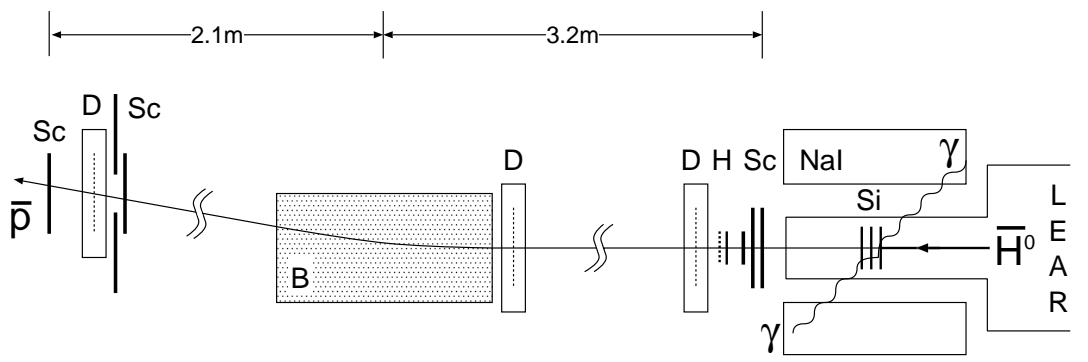

Figure 2a

Experiment PS210 : schematical top view

Sc: Trigger and time-of-flight scintillators, Si: Silicon counters, D: Delay wire chambers $\mathrm{Nal}$ : six-fold Nal-calorimeter, H: scintillating fibre hodoscope, B: magnetic dipole field

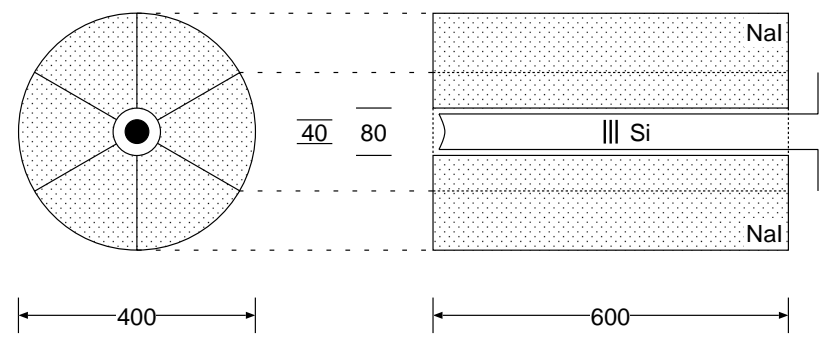

Figure $2 b$

Experiment PS210 : $\mathrm{e}^{+}$detecting section

Si: Silicon counters, Nal : six-fold Nal-calorimeter

Figure 2

Figure 1.7: Diagram of PS 210 apparatus [5]. 


\section{Chapter 2}

\section{Antiproton Beam and Target}

The apparatus is composed of three parts which are quite distinct because of their technical functions, the logistics of operating them and their location. The first, the Antiproton Accumulator, is the synchrotron ring at Fermilab where the antiprotons, created as described below, are stored. The gas jet serves as the target, and where it crosses the path of the beam, a myriad of high energy interactions take place. Finally, downstream are the E862 beamlines in which antihydrogen is stripped and its products guided into the detectors.

The E862 experiment shared the resources of the antiproton circulating beam and the gas jet target with experiment E835, a charmonium spectroscopy experiment. The two experiments took data concurrently. Discussion of the goals and impact of E835 on E862 is found in section 2.3.

\subsection{Antiproton Accumulator}

\subsubsection{Antiproton Production and Cooling}

Negative Hydrogen ions $\left(H^{-}\right)$are produced by an electric arc in hydrogen gas within the electrodes of a Cockroft-Walton electrostatic accelerator, from which they 
KEG Mar 3 , 1995
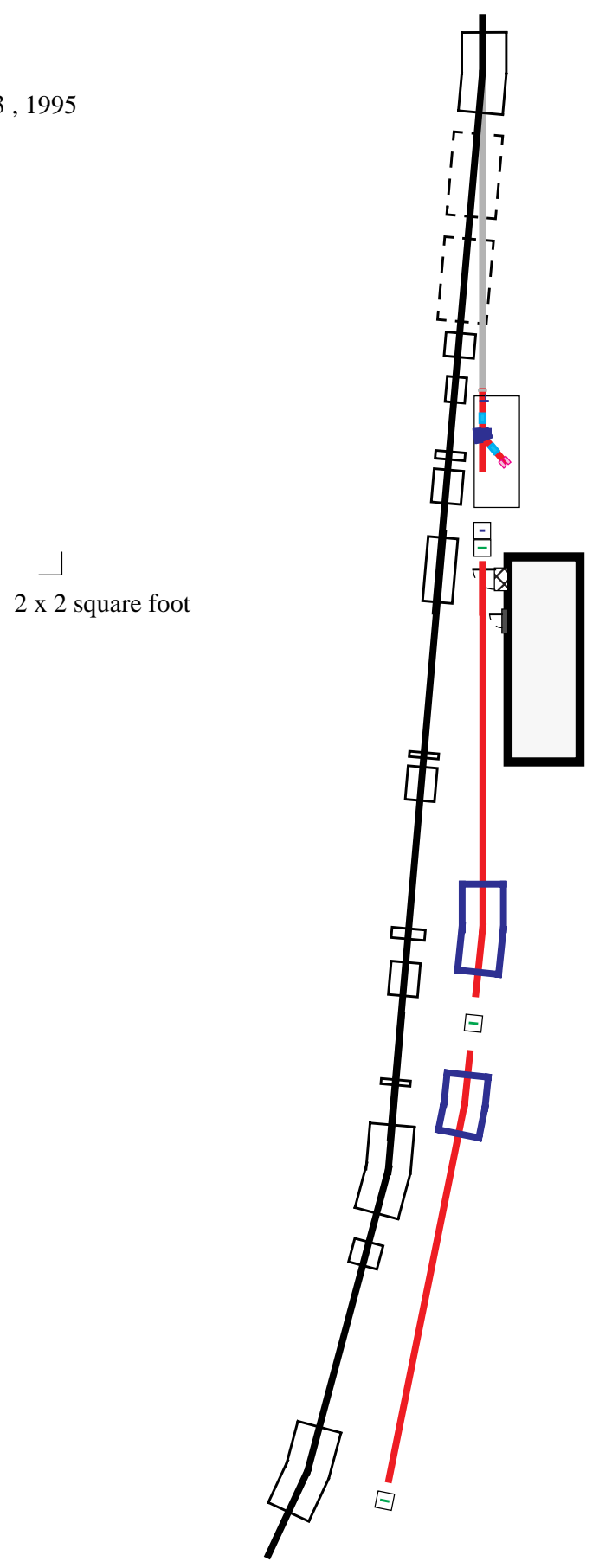

$\theta$

Figure 2.1: Placement of E862 equipment in the tunnel, to scale. 
leave at an energy of $800 \mathrm{keV}$ [67]. The ions are accelerated through the radio frequency Linac up to $200 \mathrm{MeV}$ and injected into the Booster ring operating at $8 \mathrm{GeV}$. During injection, they are each stripped into a proton and two electrons by a foil similar to the one used to strip antihydrogen, (see section 3.2). The protons are bunched in the Booster into a batch consisting of $82 \mathrm{rf}$ bunches which are injected into the Main Ring every 2 seconds, where they eventually reach $120 \mathrm{GeV}$. While the beam is in the Main Ring, the rf is used to rotate the longitudinal emittance profile of the beam so that the bunch length becomes $0.7 \mathrm{nsec}$ and the energy spread becomes 185 $\mathrm{MeV}$.

Bunches of $2 \times 10^{12}$ protons at $120 \mathrm{GeV}$ from the main ring impinge on a metal target made of either copper or tungsten. The antiprotons are produced with a small time (bunch) structure and small beam diameter matching the protons hitting the target. The $120 \mathrm{GeV}$ proton beam energy produces a spectrum of antiprotons with a maximum energy of of $10 \mathrm{GeV}[67]$. Negatively charged particles are chosen with a sweeping dipole. Afterwards, a lithium lens with very high instantaneous magnetic field, produced by passing a high current through a rod of lithium metal for a few $\mu \mathrm{sec}$, focuses the particles with momentum near $8.85 \mathrm{GeV} / \mathrm{c}$ into the injection line. Antiprotons of $8 \mathrm{GeV}$ kinetic energy are chosen since this is the energy of the booster and during startup studies, booster protons are used to test accumulator functions. The $8.85 \mathrm{GeV} / \mathrm{c}$ particles injected into the Debuncher ring include muons and pions formed in the target as well as the antiprotons. While in the Debuncher, the pions and muons (mostly from decays of the pions) decay within a few thousand turns and do not get into the Accumulator. The antiproton yield from the target is $7 \times 10^{-7} \bar{p}$ per proton. The Debuncher reduces the longitudinal momentum spread from $3 \%$ to about $0.2 \%$ through rf manipulations, and the transverse emittance from $20 \pi \mathrm{mm}$ mrad to $7 \pi \mathrm{mm}$-mrad using stochasting cooling. The antiprotons are transferred to the Accumulator shown with the Debuncher in figure 2.2. 
The longitudinal momentum spread of the antiprotons in the Accumulator is further stochastically cooled to $\frac{\delta p}{p} \leq 2 \times 10^{-4}$ and the transverse emittance is reduced to $2 \pi \mathrm{mm}$-mrad (area in phase space containing $95 \%$ of the beam) corresponding to a beam diameter of $6.4 \mathrm{~mm}(4 \sigma)$ at the interaction point. The beam is moved from the injection orbit to near the core orbit by ramping the radio frequency acceleration cavities to higher frequency. The beam is then moved onto the core with the stochastic cooling system over a period of hours, building up what is known as the stack. Beam currents up to $120 \mathrm{~mA}$ are used $\left(1.2 \times 10^{12} \bar{p}\right)$ but the beam lifetime decreases when large intensity stacks are kept and E835's data acquisition (see section 2.3) is swamped by large trigger rates. Thus most stacks were built up to $80 \mathrm{~mA}$. More detailed description of the procedures is found in [68].

\subsubsection{Deceleration Procedure}

The antiproton source rings were designed for a momentum of $8.85 \mathrm{GeV} / \mathrm{c}$. In order to get to smaller beam momenta, the radio frequency cavities must be ramped down to the appropriate frequency while simultaneously ramping the magnets accordingly. Part or all of the accumulated beam can be lost during deceleration, especially during transition crossing, due to instabilities and scraping. Since it takes approximately 24 hours to build a typical antiproton stack ( $~ 3 \mathrm{~mA} /$ hour), deceleration is a tender procedure and can take as long as 8 hours to perform. After perfecting the ramp curves during pre-data-run testing, the accelerator personnel used the ramps nearly automatically. The problem for lower energies is crossing the transition energy of the machine. At $P_{\bar{p}}=5.19 \mathrm{GeV} / \mathrm{c}$, the rf phase of a particular $\bar{p}$ with momentum deviation $\delta p$ reverses and the beam can be lost since the particles at the front of the bunch want to trade places with the those at the back at the bunch. A method of getting around this energy point involves ramping the rf to near the transition point, 


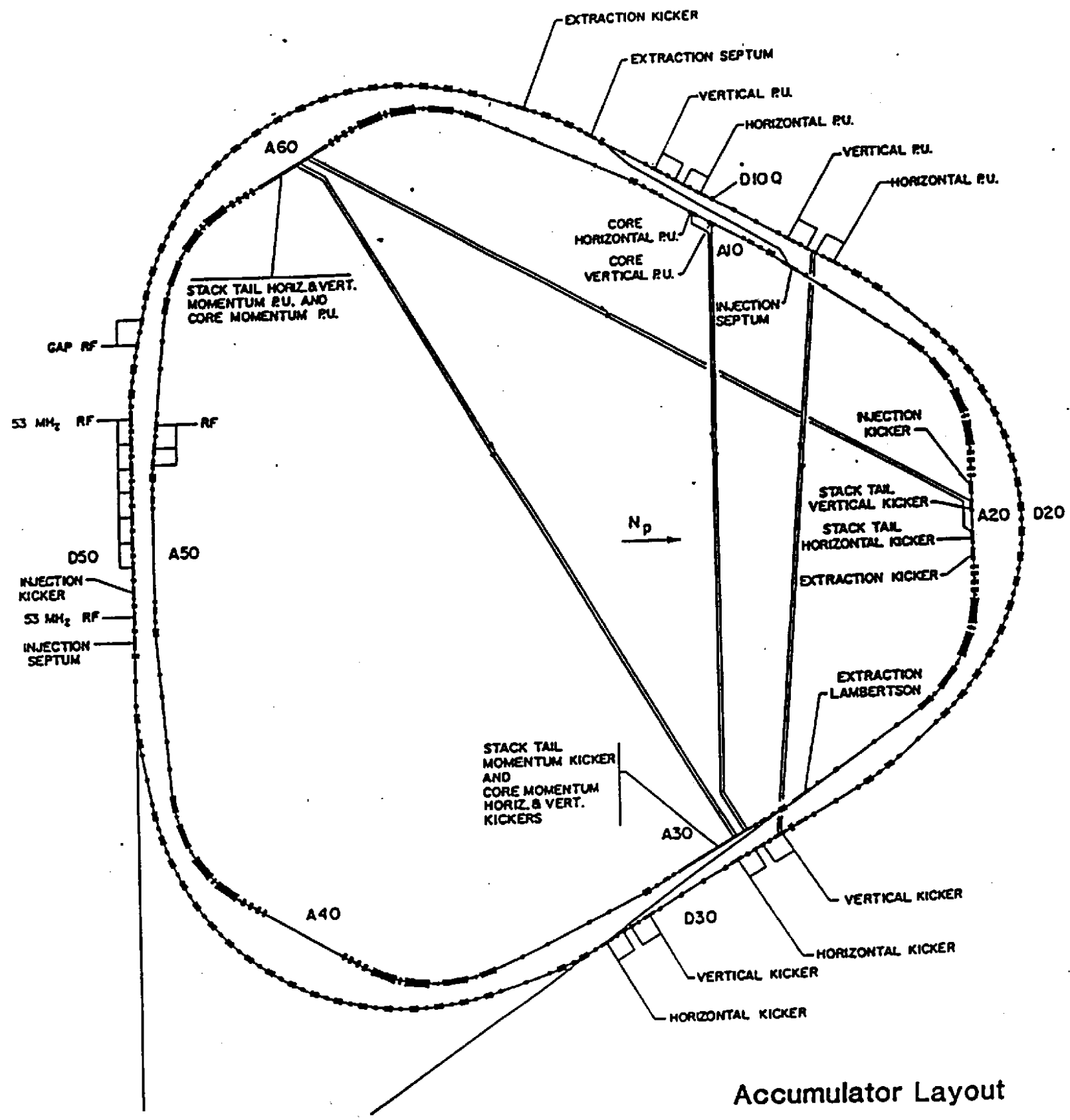

Figure 2.2: Fermilab Antiproton Source rings showing the transfer lines for stochastic cooling. A50 is the position of the jet target. The beam travels clockwise. (Figure was adapted from [67].) 


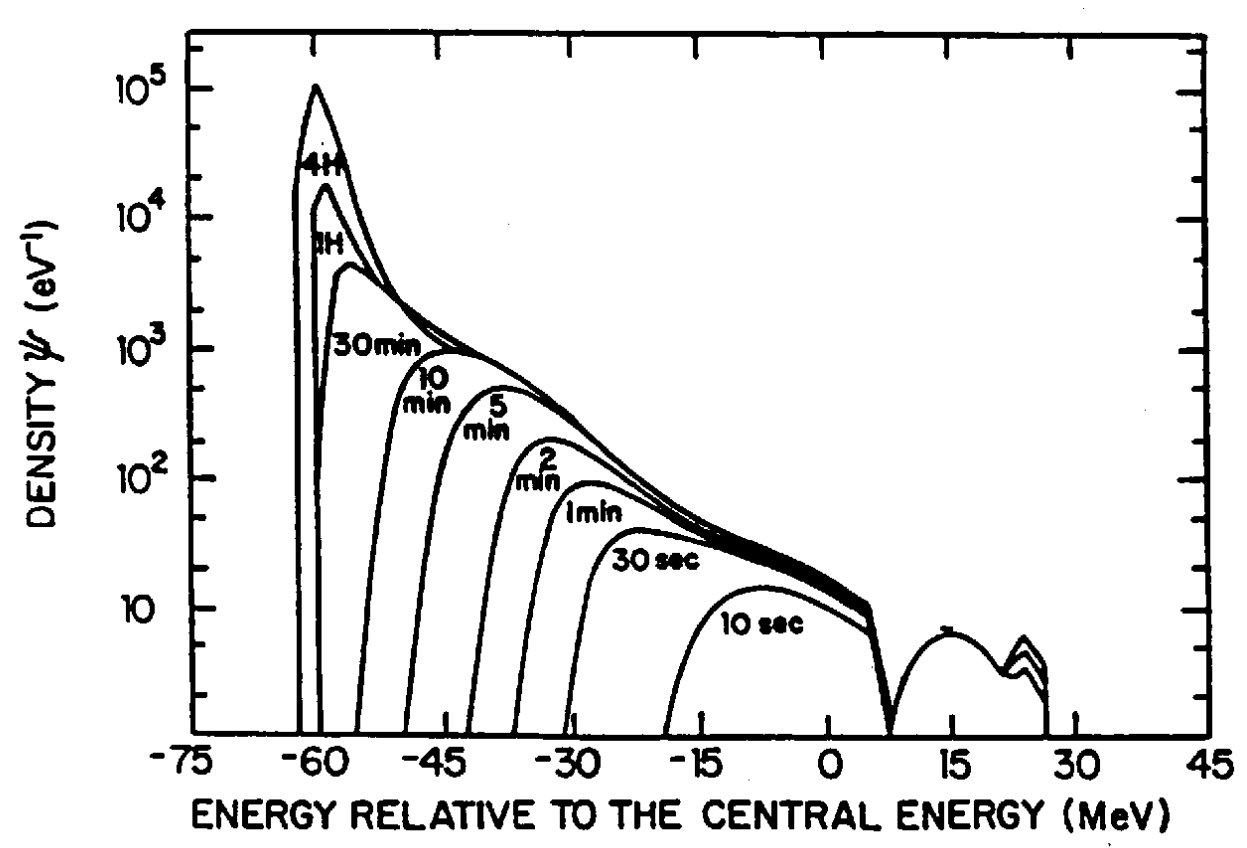

Figure 2.3: Antiproton density over time vs. orbit position (or energy) showing how stacking builds up the population at the position of the core orbit. Notice the vertical scale is logarithmic. (Figure adapted from [68]).

changing the magnet fields entirely such as to move the beam onto a different orbit, and then adjusting the rf to match up with it again. This procedure incurs beam losses of around $15 \%$.

The rate at which the magnets and rf can be ramped is only about $20 \mathrm{MeV} / \mathrm{sec}$ since the magnets are in saturation at the beginning of the ramps and thus must be allowed to settle for each step.

\subsubsection{Energy Measurement}

In order to make use of the $\bar{p}$ longitudinal momentum resolution, $\frac{\delta p}{p}=2 \times 10^{-4}$, for rejecting antiprotons, the energy and hence momentum must be measured using knowledge of the final orbit after deceleration. Since the frequency, $f$, is measured to better than $10^{-7}$, measuring the orbit length, L will determine the value and spread 
of the beam energy.

$$
\beta=\frac{f * L}{c}, \quad E_{\bar{p}}=\frac{m_{\bar{p}}}{\sqrt{1-\beta^{2}}}
$$

But $\delta L / L$ is related to the deviation in the velocity:

$$
\frac{\delta f}{f}=\frac{\delta \beta}{\beta}-\frac{\delta L}{L}=\frac{\delta \beta}{\beta}-\frac{\gamma^{2}}{\gamma_{t}^{2}} \frac{\delta \beta}{\beta}
$$

Since the energy is related to the velocity, the energy spread is:

$$
\frac{\delta E_{\bar{p}}}{E_{\bar{p}}}=\frac{\beta^{2}}{\eta} \frac{\delta f}{f}
$$

where the slip factor

$$
\eta \equiv \frac{1}{\gamma^{2}}-\frac{1}{\gamma_{t}^{2}}
$$

represents how close the energy is to the transition point.

The power spectrum (as a function of frequency) is detected by picking up the Schottky noise from a coaxial $\lambda / 4$ cavity receiver with a natural frequency at 79.323 $\mathrm{MHz}$, the 128th harmonic of the beam revolution frequency, and quality factor, $\mathrm{Q}$ $=305$ [69]. The frequency spectrum, (a typical spectrum is shown in figure 2.4), is examined with a spectrum analyzer and fit to a Gaussian plus exponential tail. The Schottky noise spectrum arises because the beam is made up of discrete particles which have slightly different momenta. Each harmonic of the revolution frequency is spread out by $\delta f / f$.

The orbit length is calculated by measuring the length of a reference orbit at the momentum corresponding to the $\psi^{\prime}$ charmonium resonance, $P_{\bar{p}}=6.23 \mathrm{GeV} / \mathrm{c}[69]$. The reference orbit is measured to $0.7 \mathrm{~mm}$ in 474 meters due only to the uncertainty in the $\psi^{\prime}$ charmonium resonance's mass (see [13] for charmonium data) and that of the much more accurate frequency measurement. To take into account the fact that the beam is not exactly on the reference orbit for some energy point, 48 beam position monitors are examined and compared for the two orbits to get an orbit length difference to an accuracy of $1 \mathrm{~mm}$. 


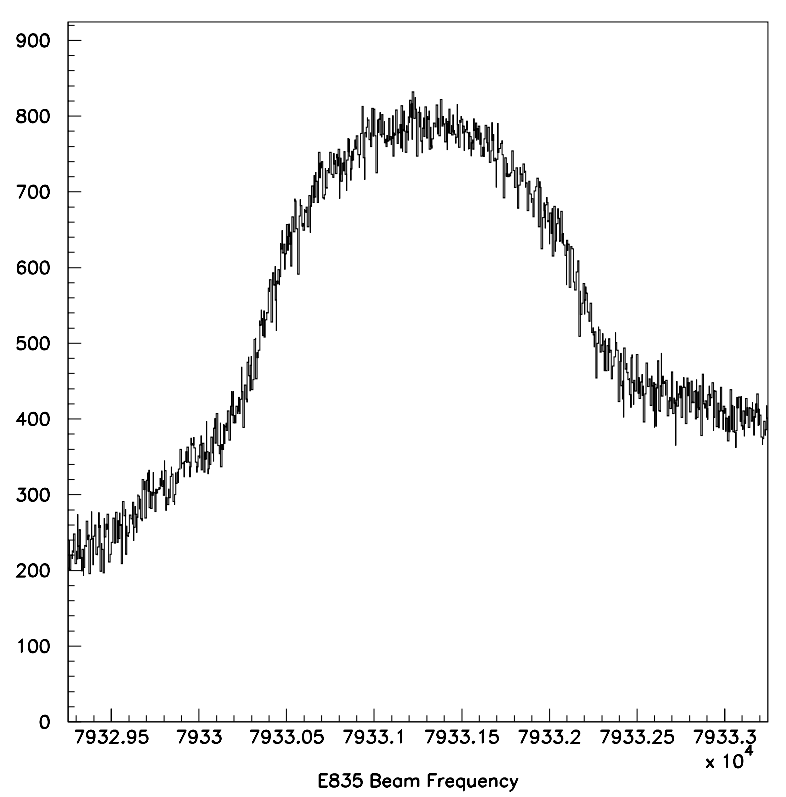

Figure 2.4: Typical frequency histogram of the beam at the 128th harmonic.

The value of the slip factor, $\eta=\eta\left(E_{\bar{p}}\right)$, at one energy is determined during the double scan of the $J / \psi$ resonance by comparing energies and frequencies at the reference orbit and a "side" orbit[69]. Since dependence of $\eta$ on energy is known, (see equation 2.4), it is also a measurement of the transition energy. The measurement of the energy depends only on very precise frequency measurements and the resonance masses [69].

The frequency spectrum and various other beam parameters such as beam positions, beam current, etc. are measured and saved to disk at regular intervals using the ACNET (accelerator network system). The ACNET system was also used to save gas jet measurements and to control and monitor E862-specific hardware such as magnet currents, (see section 5.3). 


\subsection{Gas Jet Target}

Experiment E862 uses as a production target the E835 hydrogen gas jet. The jet provides a dense beam of hydrogen molecules with minimal background gas in the vacuum of the accumulator ring besides the jet itself. We desire to maximize the number of interactions with a vertex at the origin as compared to the number taking place away from the jet. The ratio of the number of atoms $/ \mathrm{cm}^{2}$ in the jet to the number of atoms $/ \mathrm{cm}^{2}$ in the background gas, the jet target efficiency or JTE, is around $90 \%$. If antihydrogen is produced upstream or downstream of the jet, it will still have the correct trajectory to be detected.

Hydrogen at a temperature of 27 degrees Kelvin, (see figure 2.6), is injected into the first pressure chamber, J1, (see figure 2.8)[70]. There are three production stages $(\mathrm{J} 1, \mathrm{~J} 2, \mathrm{~J} 3)$ which have successively smaller pressures, the last being close to the high vacuum of the Accumulator. Entering each new stage, the molecular beam is collimated by successively larger apertures beginning with a nozzle 37 microns wide at its thinnest. Since the molecules are very near to the separation line of two phases (liquid and gas), they easily form clusters of $10^{4}-10^{6}$ molecules. At the interaction point, the transverse jet diameter is $6.3 \mathrm{~mm}$ (full width at $10 \%$ height) [71]. Across the beam, the leftover molecules are collected by three recovery stages $(R 1, R 2, R 3)$.

The path in pressure-temperature phase space must be controlled precisely so as not to condense the gaseous hydrogen $(\Delta T= \pm 0.1 K, \Delta P= \pm 0.035$ bar $)$. On the other hand, a large range of densities was desired in order to keep the luminosity constant as the beam current decreases. Nozzle temperature is measured with a germanium resistance thermometer [72] and read out with a temperature controller. The pressure at the gas inlet is measured and controlled with similar precision. The position and angle of the nozzle can be controlled to align the jet through its apertures. 


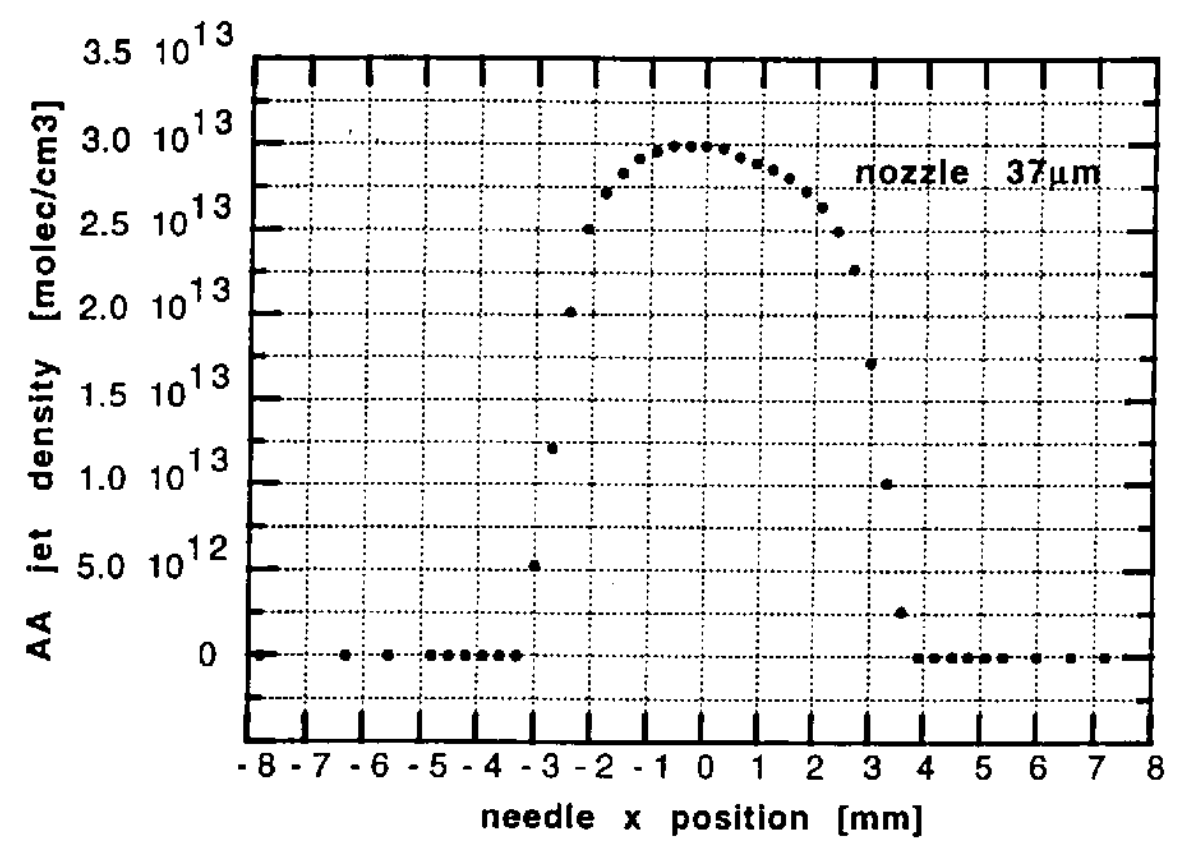

Figure 2.5: Hydrogen jet transverse density profile $19 \mathrm{~mm}$ closer to the nozzle than the interaction point [72].

For each store, the jet is left fixed while the beam is aligned with the jet. The average density of the jet can be found from the flux (molecules/sec) $\phi$, the area A, and the speed $\mathrm{V}$ of the clusters (about $600 \mathrm{~m} / \mathrm{sec}$ depending on $\sqrt{T}$ ):

$$
\rho=\phi /(A * V)
$$

Density, cluster speed, and efficiency, of the jet are calculated from the P-T operating point and read out over ACNET, (see section 2.1.3). Most of the time, the jet density was increased automatically as the beam current decreased so that a constant instantaneous luminosity was used. The density used was in the range $0.5<\rho<$ $2.3 \times 10^{14} \mathrm{atoms} / \mathrm{cc}$ or an instantaneous luminosity, $1<\mathcal{L}<4 \times 10^{31} \mathrm{~cm}^{-2} \mathrm{sec}^{-1}$.

The flux of clusters in the jet and the throughput of background gas atoms pumped in the different chambers are measured easily since they are proportional to the pumping speed times the pressure and are calibrated with a known hydrogen gas leak. The jet profile is measured with a $0.85 \mathrm{~mm}$ needle which moves in an arc 


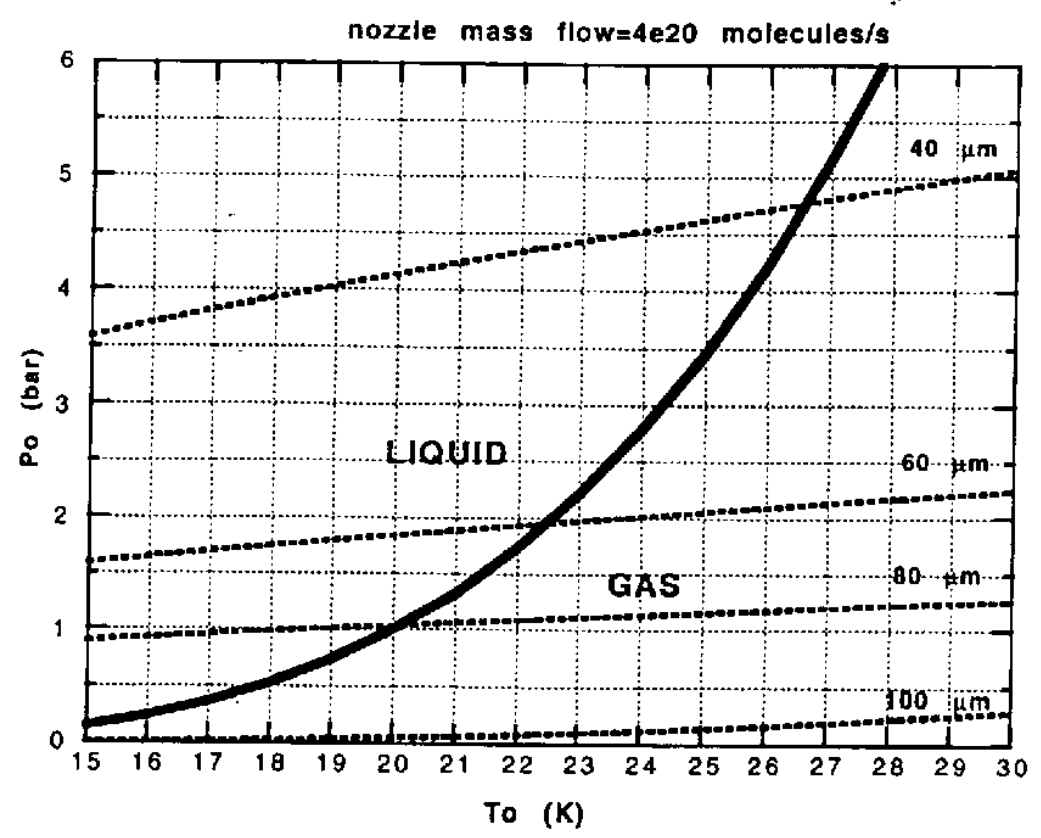

Figure 2.6: Thermodynamic phase space of the hydrogen jet's molecules. Dotted lines represent the path in phase space taken by the molecules in the jet when different nozzle diameters [72].

through the jet twice. It breaks up the clusters increasing the pressure. The jet is centered to $\pm 0.5 \mathrm{~mm}$. The cluster speed is measured by time of flight using a chopper and ion gauge separated by $850 \mathrm{~mm}$. The $\mathrm{P}, \mathrm{T}$ point is chosen to optimize the density and jet target efficiency.

When the gas jet is off, the (1/e) lifetime of the beam is about 400 hours. After the gas jet is turned on, background gas limits the lifetime to about 30-90 hours depending on the beam current. Hydrogen is used because the collision energy must be known well for E835 (see section 2.3). After antihydrogen is created, interactions with the rest of the jet as it flies through are unlikely since the dissociation cross section is $\sigma_{d i s s} \simeq 2.5 \times 10^{4}$ barns or $5.8 \times 10^{-6}$ interactions $/ \mathrm{cm}$ for the maximum jet density used. For an antihydrogen created at the upstream edge of the jet, the probability of interacting is $3.6 \times 10^{-6}$. 


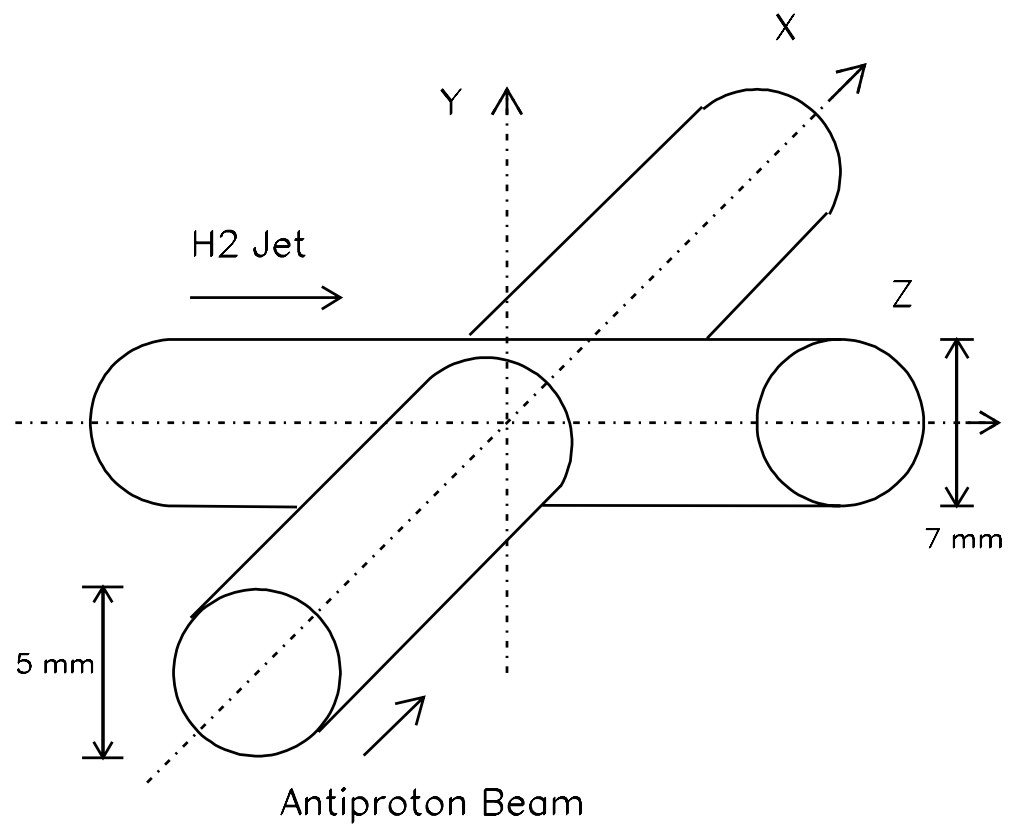

Figure 2.7: Jet-beam intersection. 

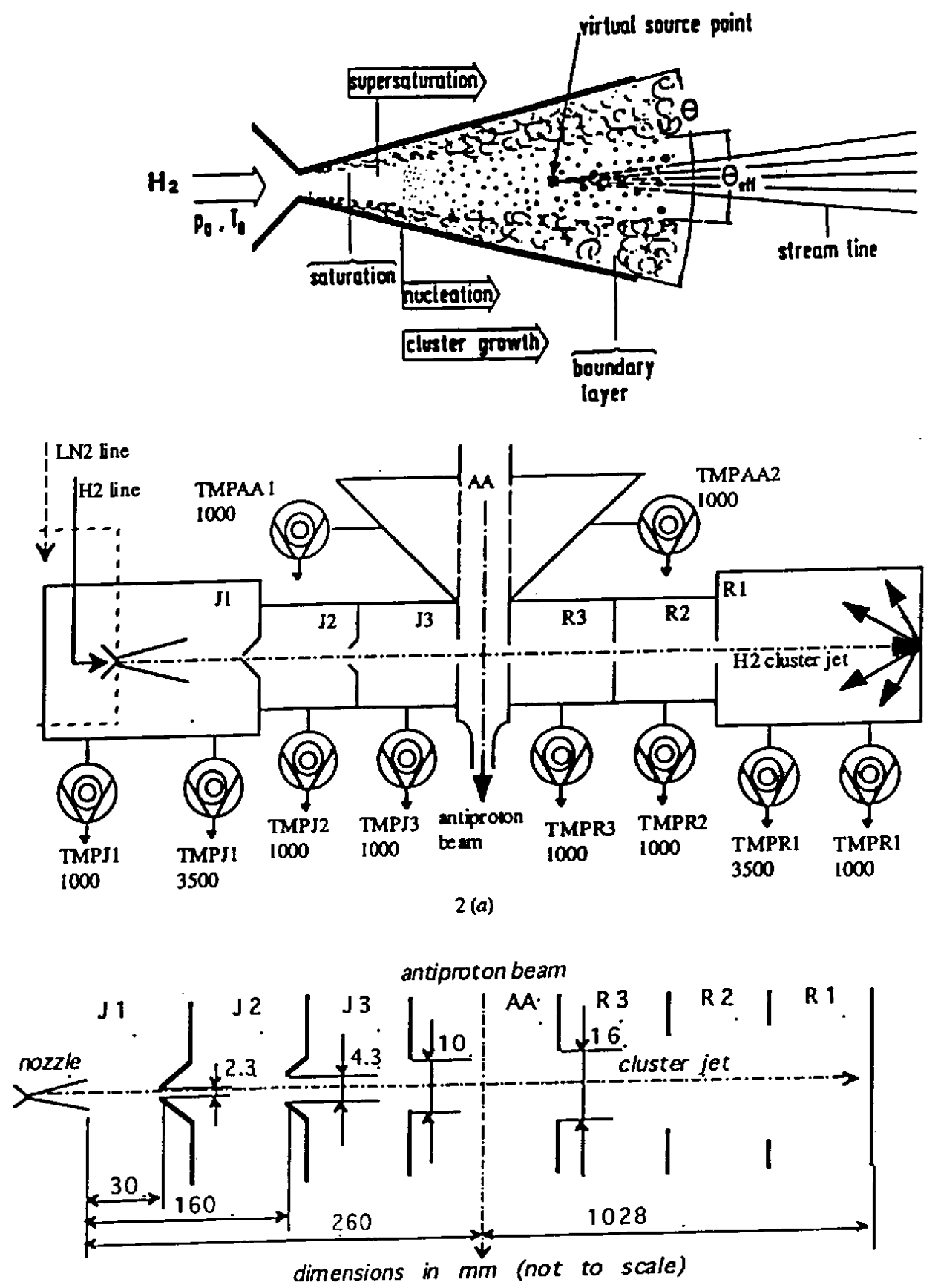

Figure 2.8: Jet target cluster formation, pumps, and chamber dimensions [72]. 


\subsection{Charmonium Program}

E862 ran as a parasitic experiment to Fermilab E835 the purpose of which was to map out the charmonium spectrum, specifically to take large amounts of data at the ${ }^{1} P_{1}$ and $\eta_{c}$ resonances and to make an exhaustive search for the $\eta_{c}^{\prime}$ resonance as well as measurements of the $\chi_{1}, \chi_{2}, J / \psi$, and $\psi^{\prime}$ resonances. The amount of luminosity accumulated at each center of mass energy point was aligned to E835's need to make precision measurements of these states. Most of the data are at a momentum around $6 \mathrm{GeV} / \mathrm{c}$. The E835 apparatus downstream of the jet target include central tracking (scintillating fibers and silicon barrel), scintillating hodoscopes, Cerenkov counter, a cylindrical, lead-glass electromagnetic calorimeter and a forward calorimeter extending roughly 10 feet from the jet. A luminosity monitor near the jet is described next. More information can be found in [69] and [73]. 


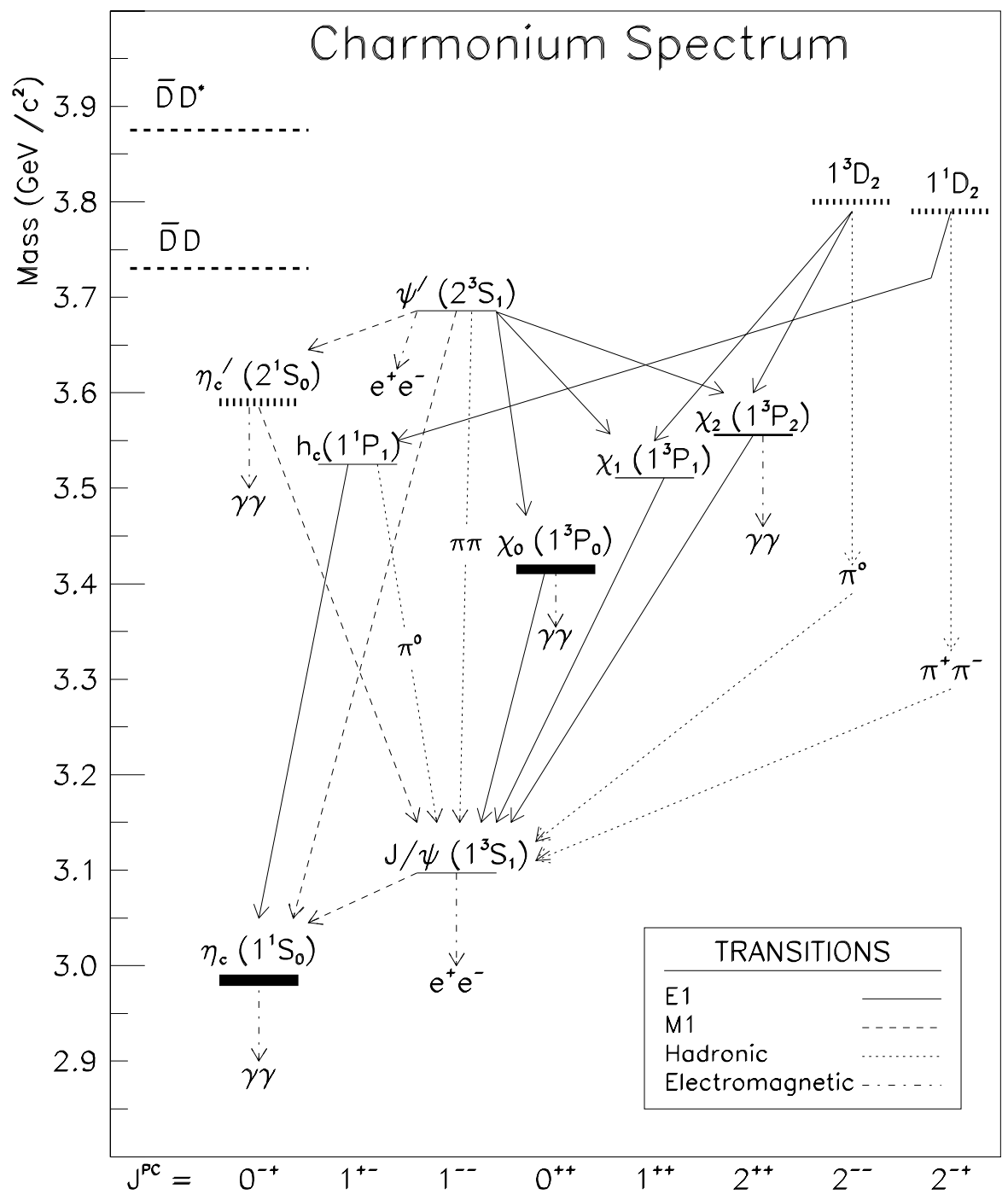

Figure 2.9: Charmonium spectrum 


\subsection{Luminosity}

Although the density of the jet is fairly well known and the intensity of the $\bar{p}$ beam is known, the luminosity calculated this way is only accurate to about $10 \%$ since the cross sectional areas of the crossing jet and beam are not known well enough. Fortunately the luminosity monitor [74] for E835 can count the recoil protons from the forward elastic scattering region. The number of recoil protons, $\mathrm{S}$, is related to the integrated luminosity but depends on the solid angle subtended and differential elastic scattering cross section at the detector position ( $t=$ four momentum transfer).

$$
\int \mathcal{L} d t=S /\left(\int \frac{d \sigma}{d \Omega} d \Omega\right) \simeq S /\left(\Omega<\frac{d \sigma}{d t} \frac{d t}{d \Omega}>\right)
$$

since the cross section is nearly independent of position across the detector (to $<$ $0.1 \%)$. This device is an ion-implanted, solid state, silicon detector and is used to count recoil protons at $3.5^{\circ}$ forward from the vertical, (see figure 2.10). The detector is located $147 \mathrm{~cm}$ below the interaction point. A histogram in energy deposit corresponding to a range of momentum transfers t, $0.0075(\mathrm{GeV} / \mathrm{c})^{2}<t<$ $0.0108(\mathrm{GeV} / \mathrm{c})^{2}$, (shown in figure 2.11), is read out using a histogramming ADC. The spectrum is fit to a proton recoil peak plus a smoothly falling background cross section. The number of events in the recoil peak are extracted from the fit compared to the known elastic differential cross section in equation 2.6 to determine $\int \mathcal{L} d t$. The detector area was calibrated with an ${ }^{241} \mathrm{Am}$ whose activity was measured to within $>1 \%$ [74]. The energy scale was calibrated with a ${ }^{244} \mathrm{Cm}$ source with two alpha emitting lines separated by $42 \mathrm{keV}$. The integrated luminosity is measured with a $4 \%$ systematic error. The error arises from imprecise knowledge of the active area, and thus subtended solid angle (2\%), and from the uncertainty in the known cross section (2\%). More detail is available in reference [74]. 


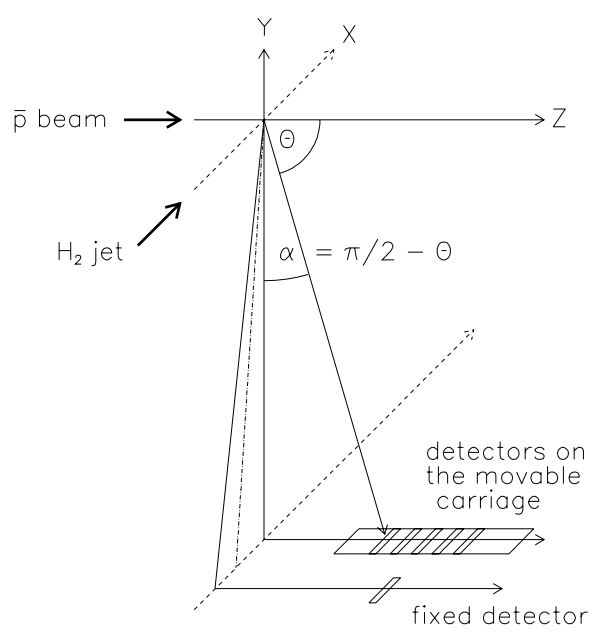

Figure 2.10: E835 luminosity monitor angle definition [74].

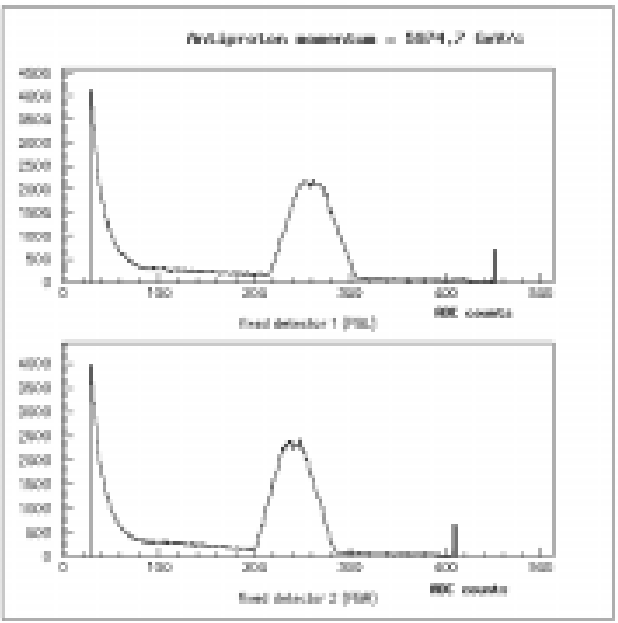

Figure 2.11: E835 luminosity monitor spectrum - for $\bar{p}$ momentum $=5974.4 \mathrm{GeV} / \mathrm{c}$ 


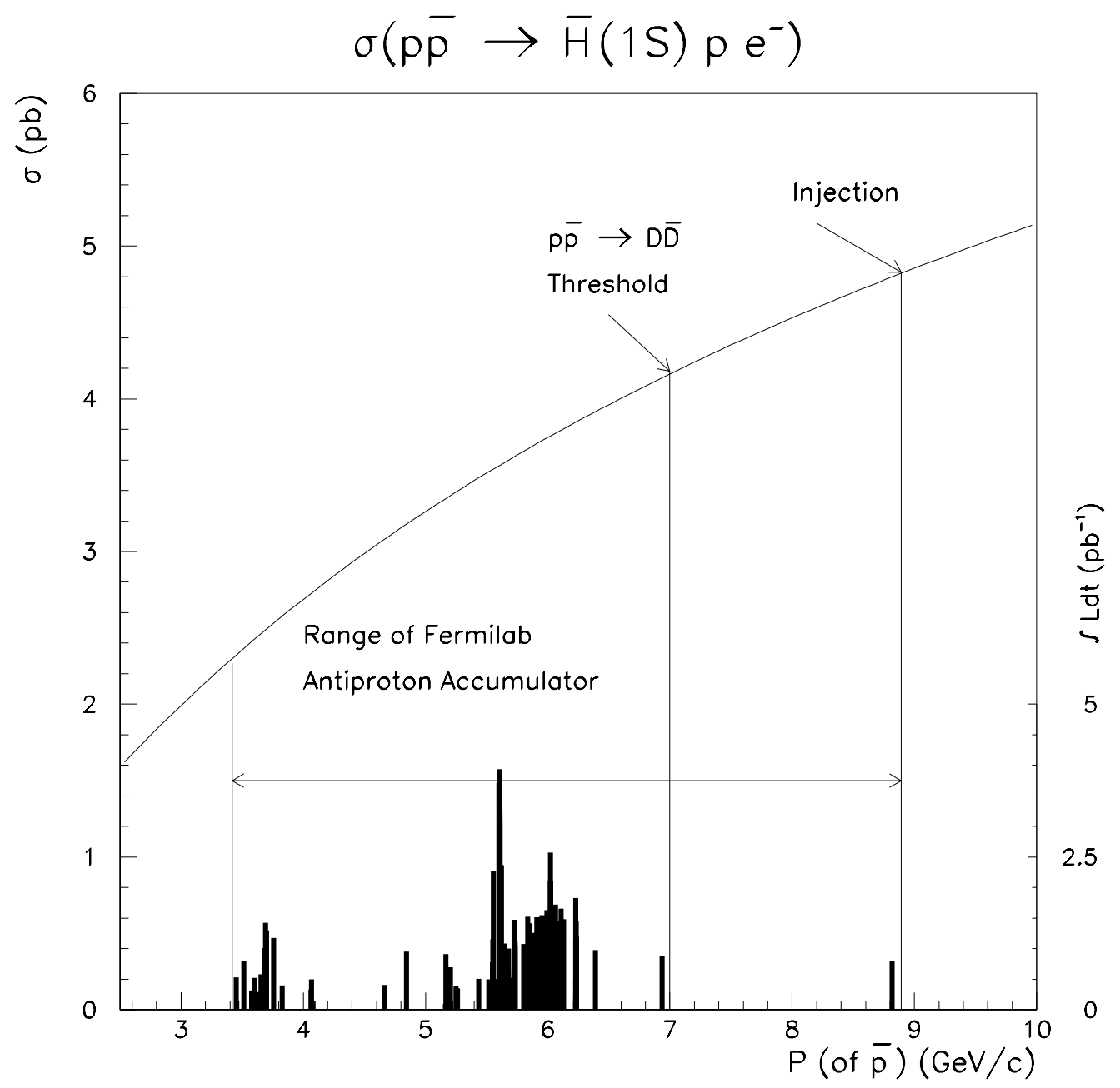

Figure 2.12: Theoretically expected antihydrogen production rate over range of momenta available. Also shown is the integrated luminosity taken by the experiment to show at which momenta the data was concentrated. 


\section{Chapter 3}

\section{The E862 Positron Spectrometer}

\subsection{E862 Detectors}

Antihydrogen atoms are created within the $<1 \mathrm{~cm}^{3}$ interaction region where the antiproton beam and hydrogen gas jet target intersect, (see figure 2.7). Downstream, after a long straight section, the first dipole magnet, (see figure 3.1), bends the antiprotons by $5^{\circ}$ as part of their trajectory around the Accumulator ring. Within the magnet, a Y-shaped vacuum pipe was installed so that the neutral antihydrogen (unaffected by the magnetic field) can continue on straight within high vacuum $\left(10^{-11}\right.$ Torr).

At a distance of 24.366 meters past the jet, a thin carbon foil ionizes the antiatoms. The velocities of the positron and antiproton are almost equal. The direction of the antiproton coincides with that of the antihydrogen. The positron is significantly multiple scattered in the foil and enters a magnetic spectrometer, consisting of solenoids for focusing the cone of positrons and a dipole which bends them by $40^{\circ}$ out of the path of the antiprotons. The spectrometer beampipe is under high vacuum and ends in a plastic scintillator plug. The positron stops and annihilates in the plug, whence the resulting back to back gamma rays are detected by a surrounding $\mathrm{NaI}(\mathrm{Tl})$ cylinder. 
The antiprotons are hardly bent by the first solenoid and dipole and continue down a beamline in partial vacuum. They are bent by two larger dipoles 40 feet downstream by a total of $10^{\circ}$. Three proportional wire chambers are placed upstream, downstream, and in between these magnets so that a momentum measurement can be made. There are also time-of-flight counters at the beginning and end of this stretch after the positron is bent away.

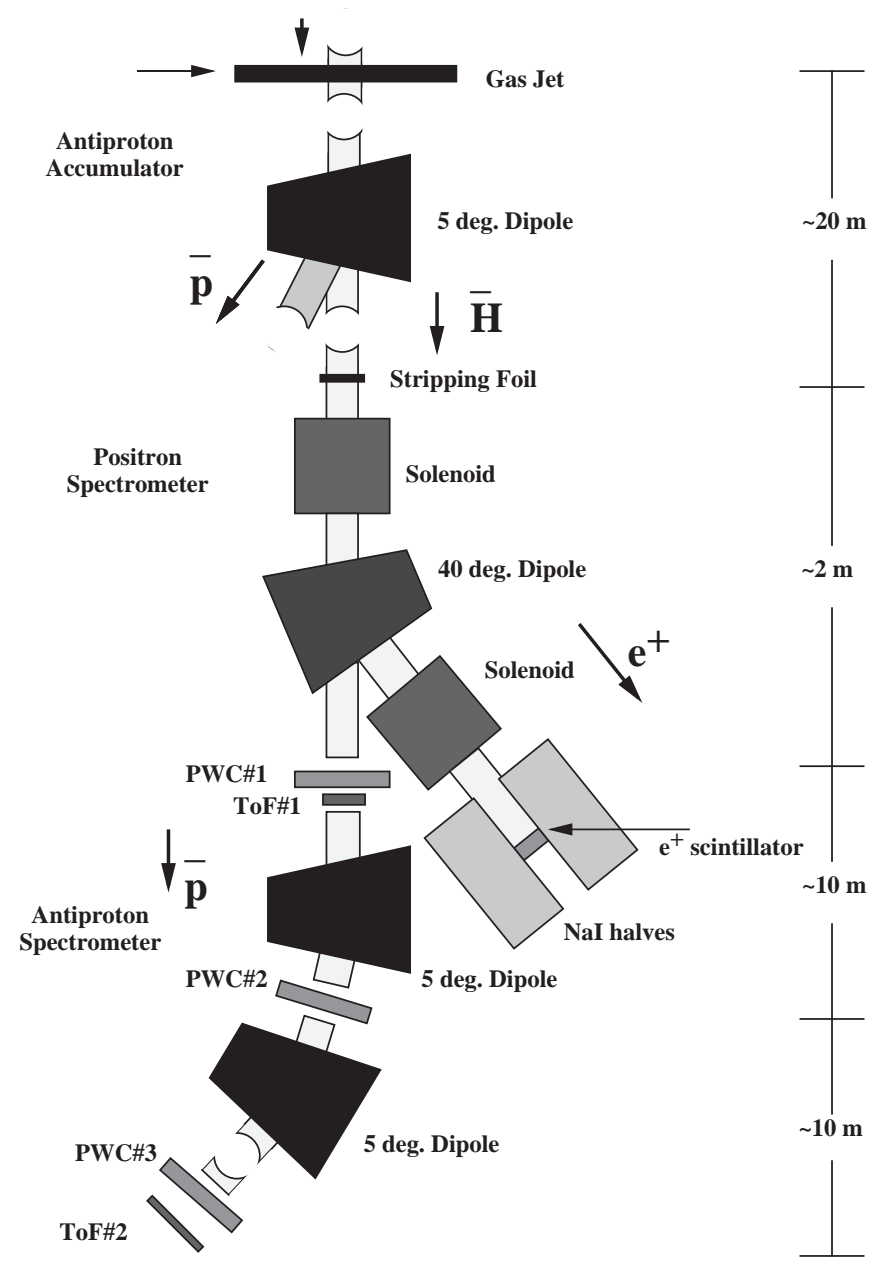

Figure 3.1: Schematic of E862 beamline elements. 
Table 3.1: Ionization rates and mean decay lengths for antihydrogen atoms

\begin{tabular}{|c||c|c|c|c|}
\hline State & $\begin{array}{c}\bar{p} \text { Momentum } \\
(\mathrm{GeV} / \mathrm{c})\end{array}$ & $\begin{array}{c}\text { A5B3 B-field } \\
(\text { Tesla })\end{array}$ & $\begin{array}{c}\text { Ionization } \\
\text { rate }\left(\mathrm{sec}^{-1}\right)\end{array}$ & $\begin{array}{c}\text { Mean decay } \\
\text { length }(\mathrm{cm})\end{array}$ \\
\hline \multicolumn{4}{|c|}{ States are, in order: $1000,2100,2010,2001$} \\
\hline 2100 & 3.5 & 0.7 & $8.3 \times 10^{-6}$ & $3.6 \times 10^{15}$ \\
2001 & 3.5 & 0.7 & $1.4 \times 10^{-4}$ & $2.1 \times 10^{14}$ \\
2010 & 3.5 & 0.7 & $2.4 \times 10^{-3}$ & $1.3 \times 10^{13}$ \\
\hline 1000 & 5.7 & 1.1 & $2.2 \times 10^{-55}$ & $1.4 \times 10^{66}$ \\
2100 & 5.7 & 1.1 & $6.9 \times 10^{9}$ & 4.4 \\
2001 & 5.7 & 1.1 & $4.4 \times 10^{10}$ & 0.68 \\
2010 & 5.7 & 1.1 & $2.8 \times 10^{11}$ & 0.11 \\
\hline 1000 & 8.9 & 1.7 & $5.0 \times 10^{-12}$ & $5.9 \times 10^{21}$ \\
2100 & 8.9 & 1.7 & $8.3 \times 10^{14}$ & $3.6 \times 10^{-5}$ \\
2001 & 8.9 & 1.7 & $2.2 \times 10^{15}$ & $1.4 \times 10^{-5}$ \\
2010 & 8.9 & 1.7 & $5.7 \times 10^{15}$ & $5.2 \times 10^{-6}$ \\
\hline
\end{tabular}

Although the antihydrogen atoms are electrically neutral, getting through the large magnetic field of the bend dipole, A5B3, is non-trivial. Most of the atoms are formed in the ground state. About $10 \%$ can be formed in excited states since the production depends on the principal quantum number roughly as $\sigma \sim 1 / n^{3}[40]$. Because the atoms are traveling at relativistic speeds, the magnetic field will be transformed into an electric field as viewed in the antihydrogen rest frame. This will cause a change in the energy levels due to the Stark effect and will increase the probability that the $e^{+}$will ionize (by tunnelling out of the potential well). Within our energy range, the ground state is unaffected. The excited states have enormously higher ionization rates. Table 3.1 illustrates how $1 \mathrm{~S}$ atoms are not affected but $\mathrm{n}$ $=2$ atoms are mostly ionized in A5B3. The notation for $\mathrm{n}=2$ states comes from expressing the hydrogen hamiltonian in parabolic coordinates which are described along with the ionization rate calculation in section B.4. 


\subsection{Foil Stripper}

The antihydrogen atoms travel down 80 feet from the gas jet in high vacuum. Connected by a bellows there is attached a vacuum enclosure which holds a $1 / 32$ " thick aluminum wheel. The wheel has 6 circular cutouts, symmetrically placed, which contain 3 stripper foils, 2 radioactive sources, and a 1" hole so that any particle could pass through, (see figure 3.4). A carbon foil about $440 \mu \mathrm{g} / \mathrm{cm}^{2}$ thick $(1 \mu \mathrm{m})$ stripped the anti-atoms into $\bar{p} e^{+}$with $99 \%$ efficiency. The other foils were a spare $440 \mu \mathrm{g} / \mathrm{cm}^{2}$ and a $777 \mu \mathrm{g} / \mathrm{cm}^{2}$ or $(1.8 \mu \mathrm{m})$ thick foil. The probability of stripping a high energy $\bar{H}$ with the $777 \mu \mathrm{g} / \mathrm{cm}^{2}$ was $100 \%$. The probability of other neutrals converting in the foil and yielding a positron is small. The foil thicknesses are determined by the manufacturer by weighing the foil and dividing by the area. Carbon is used for its ease in manufacture and resistance to thermal load (from beam heating).

The cross section, $\sigma(E)$ for stripping the ground state as a function of energy [75], is displayed in figure 3.2 :

$$
\sigma(E)=\sigma_{i o n}+\sigma_{e x c}=\frac{4 \pi a_{o}^{2}}{\beta^{2}}(Z \alpha)^{2}\left[\ln \frac{(\beta \gamma / \alpha)^{2}}{0.16}-\beta^{2}\right]
$$

which decreases exponentially with energy until a few hundred $\mathrm{MeV}$ and then decreases much more slowly. The cross section for excitation is about twice as large as the direct $1 \mathrm{~S}$ ionization but excited states strip in the foil much more easily so the effective stripping cross section is as shown.

The stripping probabilities are calculated using measurements of single and double ionization of negative hydrogen $\left(H^{-}\right)$ion beams [79],[76] $(800 \mathrm{MeV})$, and $[80],[77],[78](200 \mathrm{MeV})$. Stripping the ions with foils is used as a charge exchange injection mechanism for storage rings. These foils were used to strip $H^{-}$ions incident from the Linac at injection to the Fermilab Booster ring (200 MeV) (see section 2.1.1). A system of equations predicts the fraction of hydrogen in each charge state given 


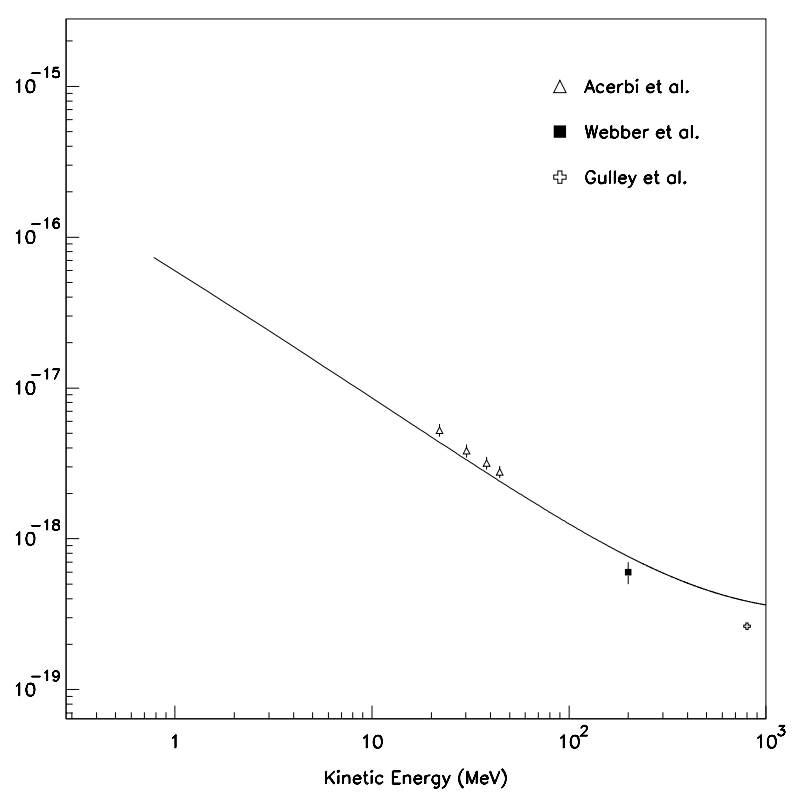

Figure 3.2: Cross section for stripping $H^{0}\left(\mathrm{~cm}^{2}\right)$. Measurements from [76],[77], [78].

the charge exchange cross sections [77]:

$$
\begin{gathered}
\frac{d N^{-}}{d x}=-\left(\sigma_{-10}+\sigma_{-11}\right) N^{-} \\
\frac{d N^{0}}{d x}=\sigma_{-10} N^{-}-\sigma_{01} N^{0} \\
\frac{d N^{+}}{d x}=\sigma_{-11} N^{-}+\sigma_{01} N^{0}
\end{gathered}
$$

The solutions for the fraction in each state as a function of the foil thickness, $\mathrm{x}$, are:

$$
\begin{array}{r}
N^{-}=e^{-\left(\sigma_{-10}+\sigma_{-11}\right) x} \\
N^{0}=\frac{\sigma_{-10}}{\sigma_{-10}+\sigma_{-11}-\sigma_{01}}\left[e^{-\sigma_{01} x}-e^{-\left(\sigma_{-10}+\sigma_{-11}\right) x}\right] \\
N^{+}=1-N^{-}-N^{0}
\end{array}
$$

The fractions were measured experimentally for various foil thicknesses in the range $10<x<300 \mu \mathrm{g} / \mathrm{cm}^{2}$. They were fit to the expected fractions using the cross sections as parameters yielding cross sections at $200 \mathrm{MeV}$ [77]. 


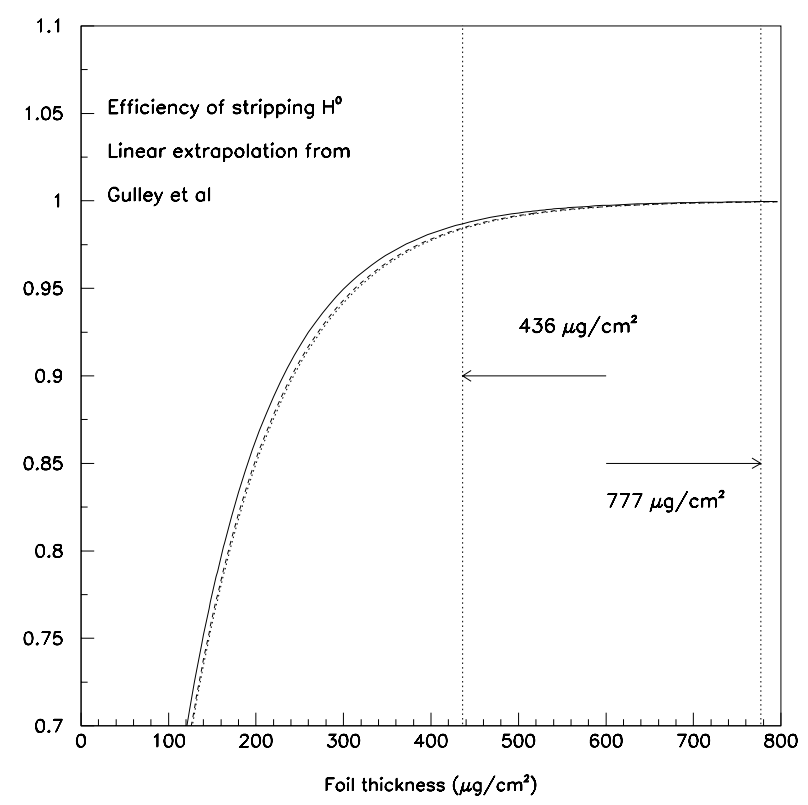

Figure 3.3: Efficiency for stripping $H^{0}$ as a function of carbon foil thickness. The solid curve is for a $3 \mathrm{GeV} / \mathrm{c}$ antihydrogen, the dashed for $6 \mathrm{GeV} / \mathrm{c}$, the dotted for 9 $\mathrm{GeV} / \mathrm{c}$.

Figure 3.3 shows the stripping efficiency of carbon for hydrogen incident energies of $3 \mathrm{GeV}, 6 \mathrm{GeV}$, and $9 \mathrm{GeV}$ as a function of thickness of a set of foils. The efficiency at these higher energies for stripping antihydrogen was calculated by linearly extrapolating the data. This included the highest energy cross section data at $800 \mathrm{MeV}$ as a worst case scenario and did not include the relativistic increase of the cross section expected in the higher energy range. The $\sim 440 \mu \mathrm{g} / \mathrm{cm}^{2}$ thickness was chosen to give $>99 \%$ efficiency.

In the case of E862, we assume that the antihydrogen has the same stripping cross section as hydrogen in the carbon foil. It is also assumed that the hydrogen atoms $\left(H^{0}\right)$ formed by charge exchange in [77] were in the ground state. The stripping cross section for excited state hydrogen atoms will be slightly larger anyway. 


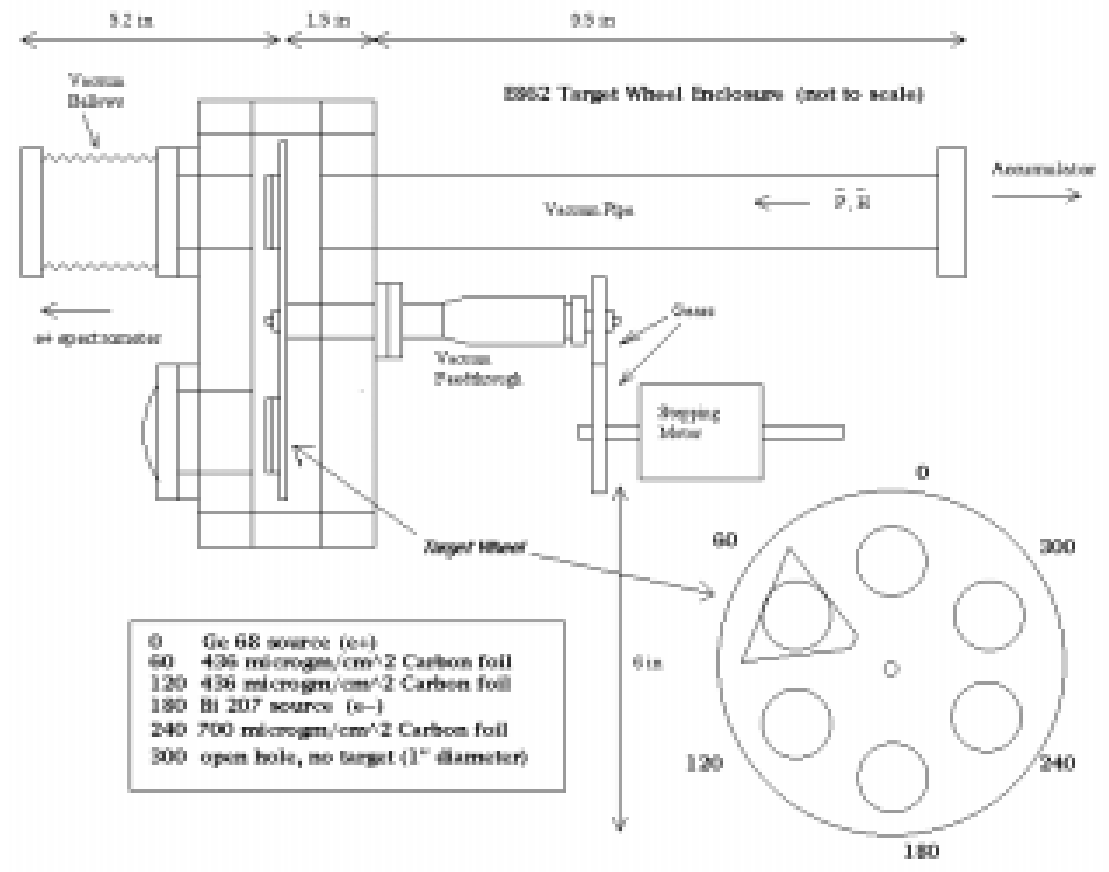

Figure 3.4: Vacuum box for target wheel.

\subsection{Radioactive Sources}

One of the radioactive sources mounted on the wheel was ${ }^{207} \mathrm{Bi}$, a monoenergetic electron source with an initial activity of $10 \mu \mathrm{Ci}$. It was used to find the correct magnetic fields for focusing the positrons. A $\beta^{+}$source, ${ }^{68} \mathrm{Ge}$ with an initial activity of $10 \mu \mathrm{Ci}$, was used to check the integrity of the path all the way through to the sodium iodide. Since the $\beta^{+}$spectrum has an energy endpoint of about $1.899 \mathrm{MeV}$ [13], the magnets had to be tuned below any field used for normal data-taking. The ${ }^{68} \mathrm{Ge}$ source was constructed of evaporated metallic salt of germanium contained within a Nickel ring with $0.0002 "$ nickel cover plates. The active area was $0.713 \mathrm{~cm}^{2}$. 
Table 3.2: Antiproton beam parameters using Fermilab standard emittance definitions. The emittance used in both dimensions is $2 \pi \mathrm{mm}$-mrad, the maximum measured.

\begin{tabular}{|c||c|c|}
\hline$\sigma_{x}$ & $\sqrt{\epsilon_{x} \beta_{x} / 6 \pi}$ & $1.57 \mathrm{~mm}$ \\
$\sigma_{\theta_{x}}$ & $\sqrt{\epsilon_{x}\left(1+\alpha_{x}^{2}\right) / 6 \pi \beta_{x}}$ & $0.21 \mathrm{mrad}$ \\
$\sigma_{y}$ & $\sqrt{\epsilon_{y} \beta_{y} / 6 \pi}$ & $1.40 \mathrm{~mm}$ \\
$\sigma_{\theta_{y}}$ & $\sqrt{\epsilon_{y}\left(1+\alpha_{y}^{2}\right) / 6 \pi \beta_{y}}$ & $0.24 \mathrm{mrad}$ \\
\hline
\end{tabular}

\subsection{Antihydrogen Beam Parameters}

It was essential to know how large the beam spot would be on the foil target and the positron scintillator, and how many wires would be needed in the chambers. Above all, knowing the antihydrogen would not hit the beampipe anywhere was of utmost importance.

The beam size distributions are characterized by the standard deviations found in table 3.4. These numbers assume both transverse emittances are equal to $2 \pi \mathrm{mm}$ mrad, an upper bound on their maximum value. The emittance in the $\mathrm{x}$ dimension, $\epsilon_{x}$ is given as the area of the phase space $\left(x, \theta_{x}=p_{x} / p_{z}\right)$ containing $95 \%$ of the particles. The y emittance is defined similarly. The Courant-Snyder parameters, $\alpha=0$ and $\beta$ are calculated from a knowledge of the lattice (field strengths, positions of the magnets around the ring) [81]. The beam has a roughly circular cross section (equal width Gaussian distributions in $\mathrm{x}$ and $\mathrm{y}$ ) with large coupling between the two transverse dimensions.

$$
\varepsilon_{x}=-2 \pi \sigma_{x}^{2} / \beta_{x} \cdot \ln (1-F)=6 \pi \sigma_{x}^{2} / \beta_{x}
$$

[81] where $\mathrm{F}$ is the fraction of particles inside the phase space ellipse (95\%). The angular spread is:

$$
\sigma_{\theta_{x}}=\sigma_{x} / \beta_{x}
$$


The beam emittance in the accumulator ring was usually less than $2 \pi \mathrm{mm}$-mrad. E862 has measured the emittance by examining the high momentum tracks which enter the E862 antiproton spectrometer. Section 6.6.4 discusses these measurements. The antihydrogen candidates' tracks clearly form a transverse emittance envelope corresponding to $\epsilon_{x}, \epsilon_{y}<2 \pi \mathrm{mm}$-mrad. This data took the whole running period to accumulate, so it does not serve as a method of monitoring the emittance during the run. The transverse emittances are usually extracted from a measurement of the betatron cooling sideband spectrum of the beam. The angular divergences, $\sigma_{\theta}$, are calculated using the beam spot size and beta function at that position of the ring. The position of the beam centroid is measured by beam position monitors.

The beam position monitors are basically semicylindrical pickup electrodes within the vacuum pipe installed between the pole faces of the quadrupole magnets. There are 48 monitors in each dimension; not all quadrupoles have a monitor. They are tuned to a harmonic of the beam revolution frequency and must have the beam partially bunched in order to receive a signal. The horizontal and vertical beam positions measured by the monitors installed in the two accumulator quadrupoles, A4Q1 and A5Q1, just upstream and downstream of the jet, were used to draw a line to the foil target. The neutral antihydrogen follows a straight path from the interaction point collinear to the beam at that point. The monitors achieved a precision of \pm 1 $\mathrm{mm}$ in both transverse dimensions. If the maximum error was encountered in the two monitors nearest the jet target, a beam steering error of $0.35 \mathrm{~cm}$ would result at the first downstream bend magnet, A5B3, where the $\bar{H}^{0}$ exits the storage ring. This is at least 4 times smaller than what would result in scraping by the beam pipe in the A5B3. During the calibration run, (see section 4.3), the beam centered in both quadrupoles was also seen to traverse a centered path through the wire chambers. 
At the position of the E835 jet target interaction point, the $\bar{p}$ beam is within a zero dispersion region such that a spread in momentum will not translate into a spread in position. Section 6.6.4 shows that the phase ellipse defining the emittance is not aligned along the $\mathrm{x}$ and $\mathrm{x}^{\prime}$ axes. This is due to a cross term, $\alpha=-\frac{1}{2} \frac{d \beta}{d s}$ in the expression for emittance[81]:

$$
\frac{\epsilon}{\pi}=\gamma x^{2}+2 \alpha x x^{\prime}+\beta x^{\prime 2}
$$

The beta function, $\beta$, is dependent on magnet configurations and fields only, and $\mathrm{s}$ is the path length of a particle.

The momentum transfer during the production is small, $\delta p_{x} / p \sim 0.181 / 3000=$ $0.06 \mathrm{mrad}$ making $\sigma_{\theta_{x}}$ larger by $\sqrt{0.2^{2}+0.06^{2}}=0.21 \mathrm{mrad}$. At the position of the foil target, the beam size at one standard deviation in $\mathrm{x}$ and $\mathrm{y}$ is $\sigma_{x}=0.53 \mathrm{~cm}$ and $\sigma_{y}=0.67 \mathrm{~cm}$ on average but depends on the emittance. If a circular beam is assumed, with $\sigma_{r}=0.6 \mathrm{~cm}$, the beam covers $22 \%$ of the foil at $1 \sigma$. Given the (nearly) Gaussian probability distribution for a hit outside the radius $r, P=e^{-r^{2} / 2 \sigma^{2}}, 90 \%$ of the beam is contained within a disk of diameter $1 "=2.54 \mathrm{~cm}$ for a beam centered on the foil. Section 7.4.1 discusses inefficiencies incurred when the beam is not centered on the foil. The A5B3 beampipe was specifically redesigned to accomodate $3 \sigma$ of the straight-flying antihydrogen beam in both the vertical and horizontal directions. Discussion of the beam spots measured during the calibration run can be found in section 4.3. The beam size was measured in March 1997 using the flying wire system above the transition energy and matched expectations based on an emittance of $1.6 \pi$ $\mathrm{mm}$-mrad in both dimensions and a $\sigma_{\theta}<0.25 \mathrm{mrad}$ angular spread at the gas jet. 

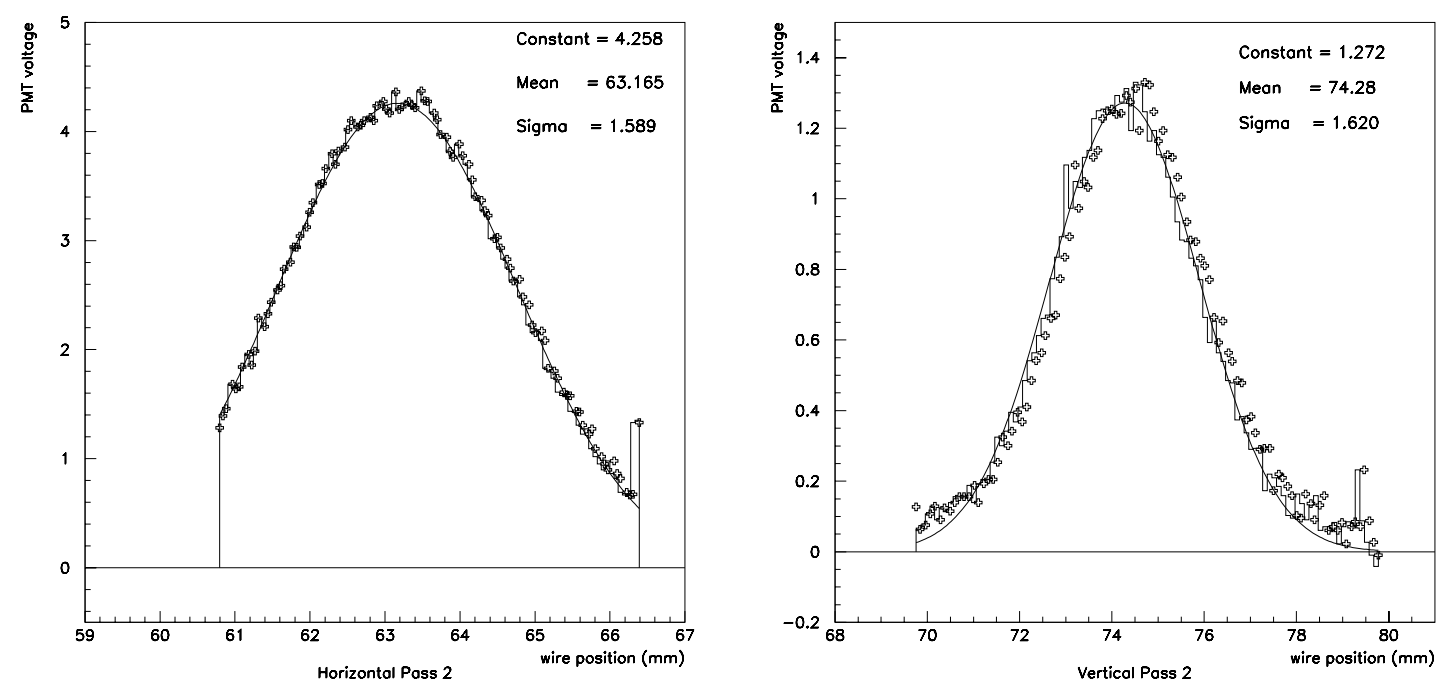

Figure 3.5: Flying wire profiles of antiproton beam at A3Q7 and A4Q14, respectively.

\subsection{Positron Magnets}

\subsubsection{Design and Simulations}

The positrons multiple scatter in the $440 \mu \mathrm{g} / \mathrm{cm}^{2}\left(10^{-3}\right.$ radiation lengths $)$ foil enough to exit within a $\theta=6^{\circ}$ cone $\left(3 \sigma_{\theta}\right)$ at $1.2 \mathrm{MeV}$. It is important to remember that the "beam" E862 uses is a very low rate source of antihydrogens. Subtle beam effects like space charge are not applicable since more than one positron is never within the spectrometer at any one time. It was desired to bend the positrons out of the way in order to detect them. This would also entail reproducing a reasonable beam spot on the final detector to ensure the hit was not from background. A small bend dipole is adequate for the application, but because the beam has an angular divergence, it is better if the dipole is able to focus positrons entering at different angles so they will converge at the exit point. 


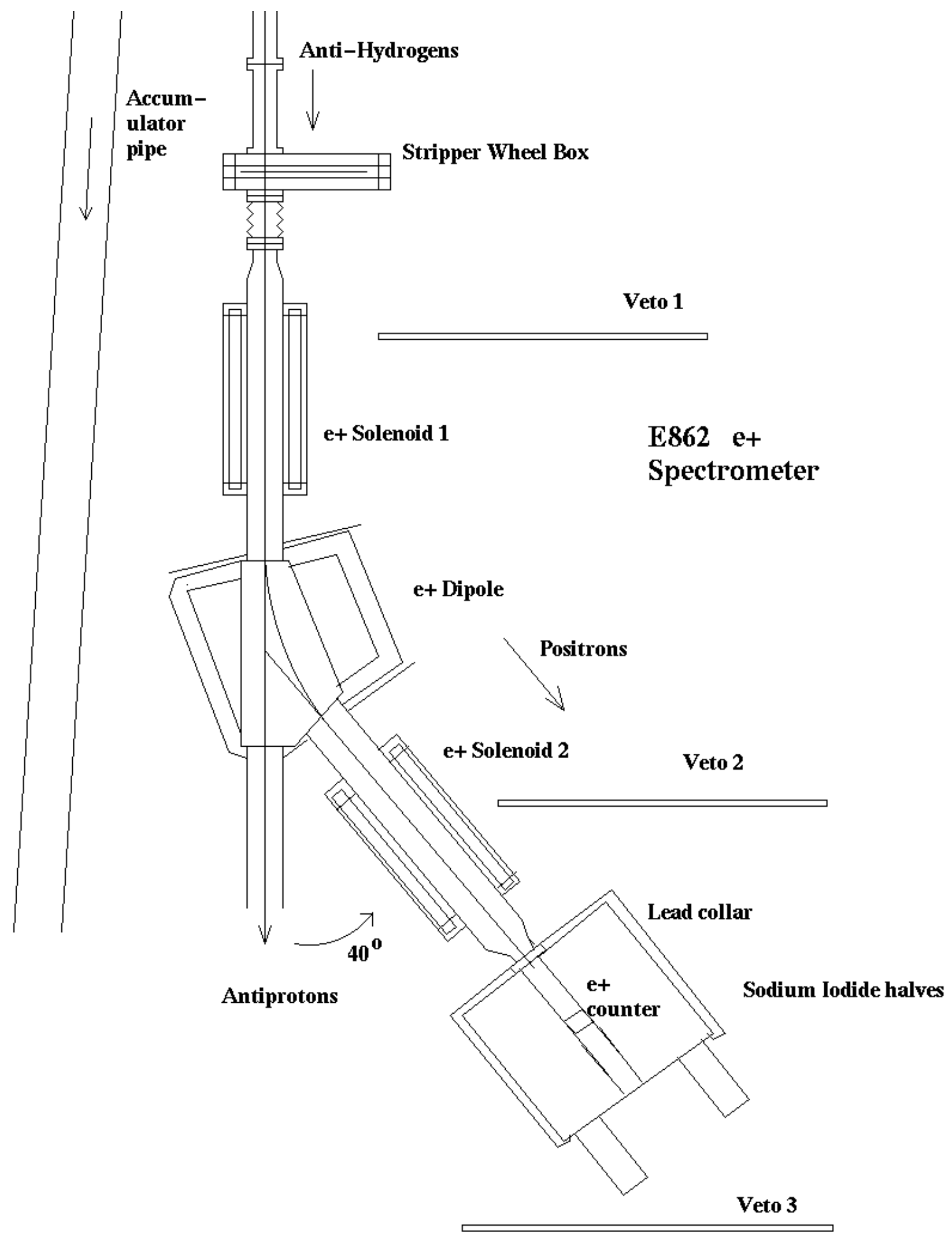

Figure 3.6: Schematic of E862 beamline elements. 


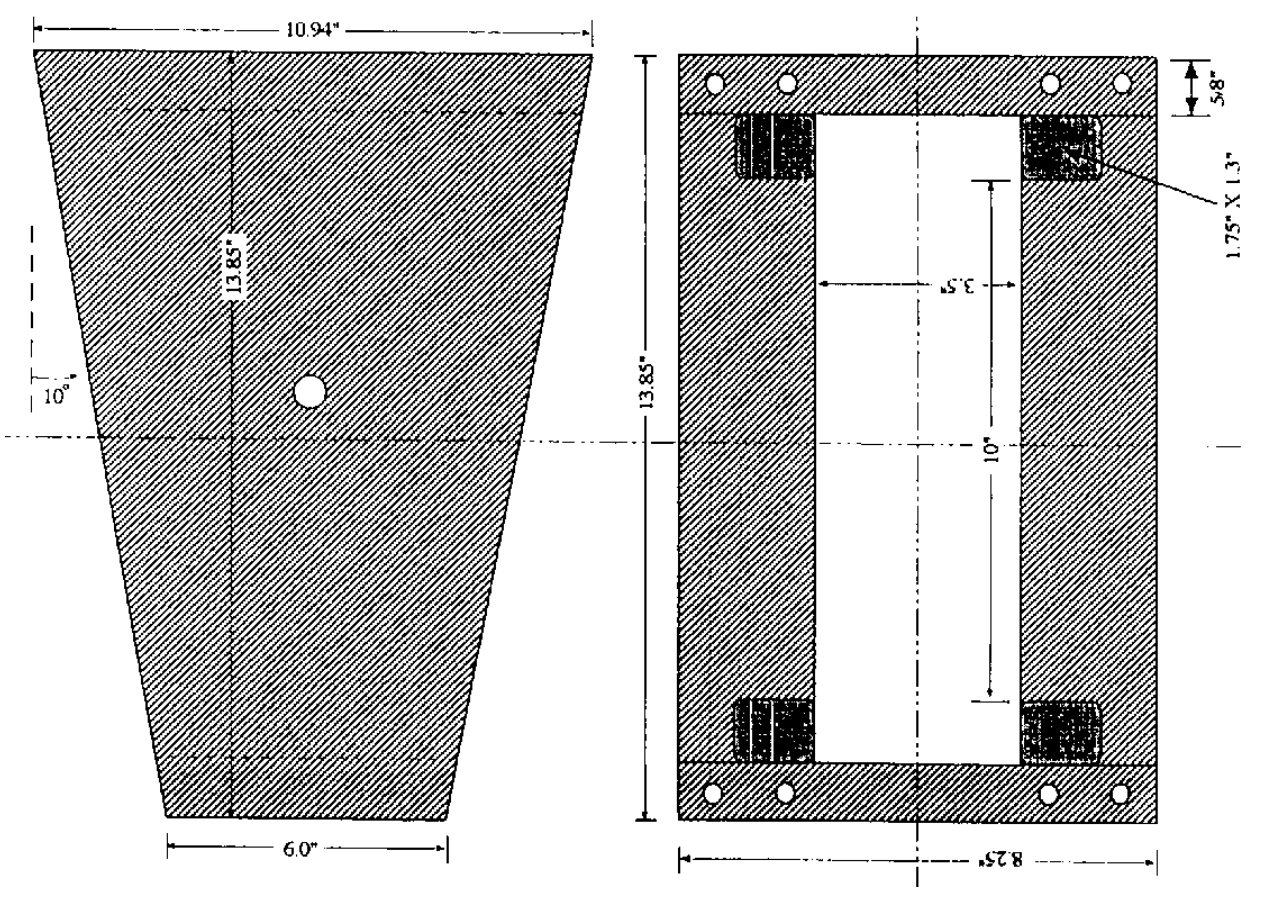

Figure 3.7: $40^{\circ}$ dipole for positrons showing flanged edges at $10^{\circ}$.

\section{Dipole Focusing}

We can characterize a sector dipole as a thin lens located at the center of the dipole. The focal length in the bend plane is equal to the ratio of the radius of curvature to the tangent of the bend angle $\alpha=40^{\circ}, f_{h}=r_{g} / \tan \alpha$. The radius of curvature is $r_{g}=p /(e B)$ where $\mathrm{p}$ is the momentum and $\mathrm{e}$ is the charge of the electron. One problem is that there is focusing in the plane of the bend but not in the normal plane. In the case of E862, the bend plane is the horizontal and the normal plane is vertical. A sector dipole having flanged edges has fringe fields that particles encounter so that vertical focusing (or defocusing) occurs. Each of the two edges can be characterized by a certain focal length, $f_{v}=r_{g} / \tan \beta$ where $\beta$ is the angle between the edge of the magnet and the central particle trajectory. When $\beta=\frac{\alpha}{4}=10^{\circ}$, the overall focal lengths of the magnet in $\mathrm{x}$ and $\mathrm{y}$ are best matched. Field plates are positioned at the entrance region to minimize the extent of the dipole fringe field. 


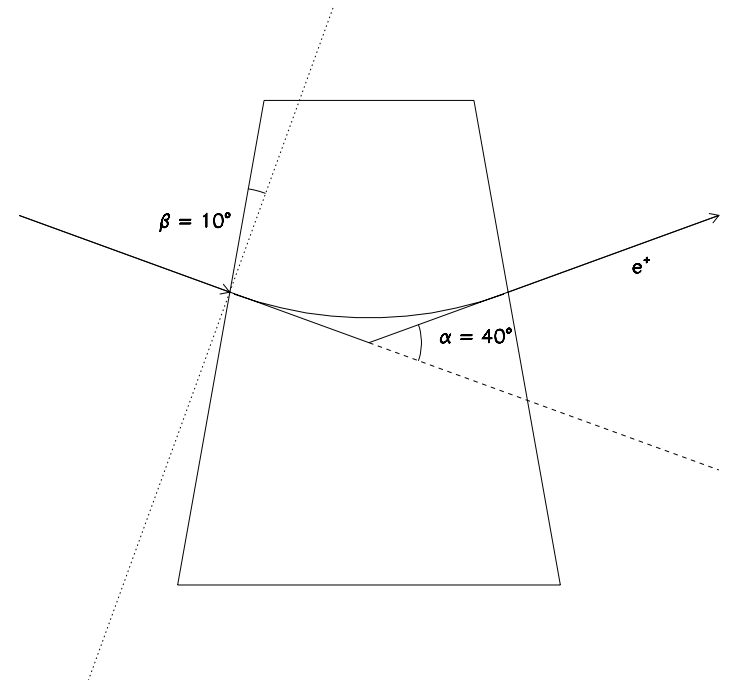

Figure 3.8: Diagram of entrance/exit angles of $e^{+}$for $40^{\circ}$ bend dipole.

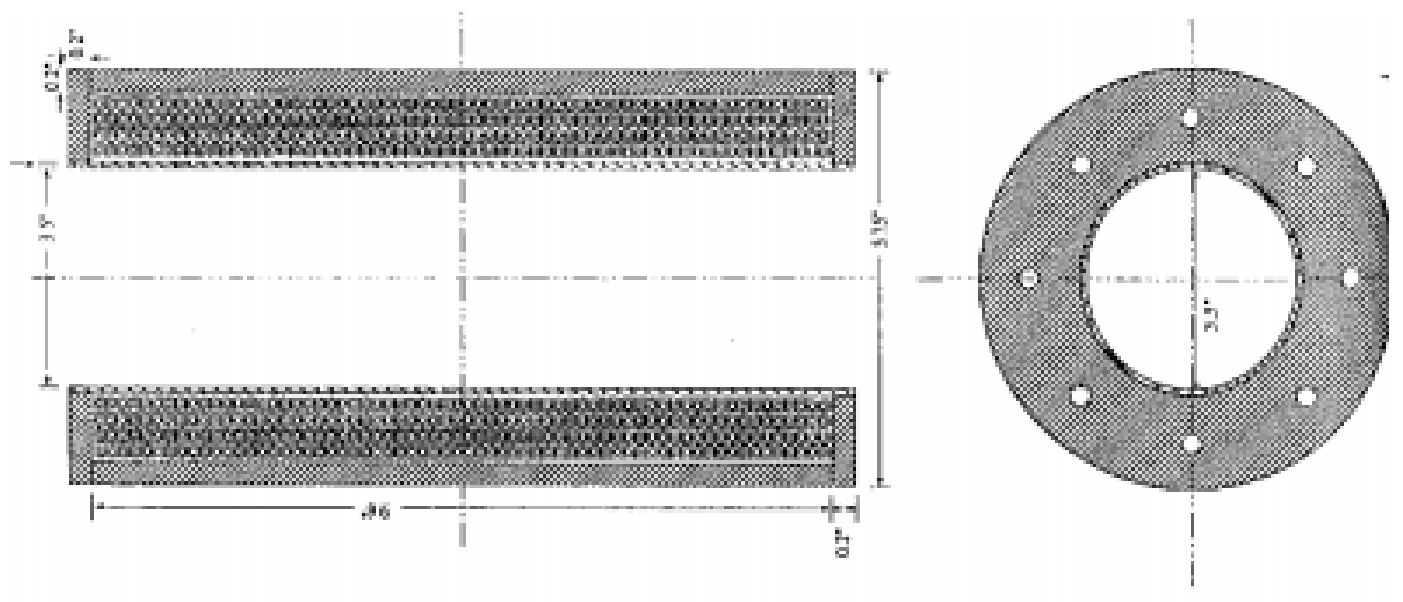

Figure 3.9: Focusing solenoid for positrons. The magnet is perfectly cylindrical. The flatness at the right edge is an artifact of a scan. 


\section{Solenoid Focusing}

Solenoids were chosen to collect the distribution of positrons exiting at large angles. Since the solenoids are symmetric about the beam axis, they are easier to construct accurately, but the focusing power of solenoids gets much worse for higher energy beams. They are often used in electron microscopes, for example, since the electron energy is relatively low. The three magnets form a symmetric, point to point focusing system. Two solutions exist which depend on the magnetic field in the solenoids. The trajectories can either cross each other at a node in the center of the dipole or not. The higher magnetic field solution causes the crossing to take place and has a higher acceptance, $\pm 7 \%$ for off-momentum positrons. The lower field solution has a $\pm 3.6 \%$ longitudinal momentum acceptance window.

The Lorentz force on the positron while its radial position is increasing within the solenoid is in a $\phi$ direction, $\phi$ being the azimuthal angle around the solenoid axis. The $e^{+}$gains a momentum component in the $\phi$ direction. This leads to a component of the force in the negative radial direction which is focusing. When the net radial component of the $e^{+}$momentum is negative (around the middle of the magnet), the force component in the $\phi$ direction changes sign. If the field is reversed, there is no difference in the focal length because the $e^{+}$receives the same changes in the radial components even though the $\phi$ motion is reversed. This difference in the $\phi$ motion is responsible for the two solenoids producing beam spots at their image plane which have different locations. This is due to the different image rotations imparted by the solenoids. If the solenoids both have a length such that both images rotate by $n \pi$ then the images have the same position in the image plane. After testing, it was discovered that misalignments necessitated the polarity switch in order to reverse the rotation of the image and thus move the beam spot. Basically, if the $e^{+}$propagates 


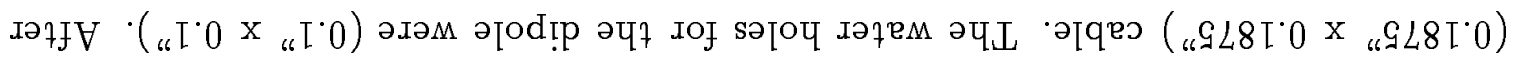

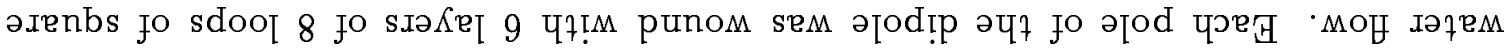

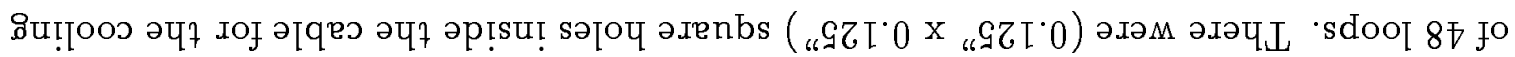

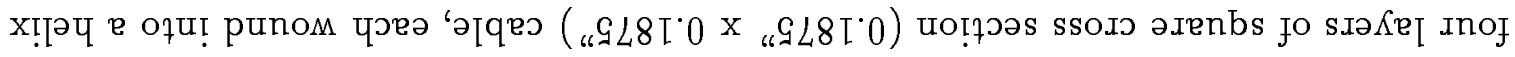

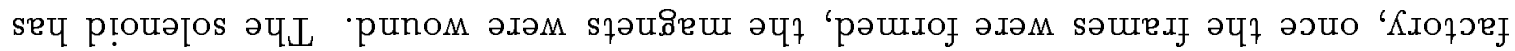

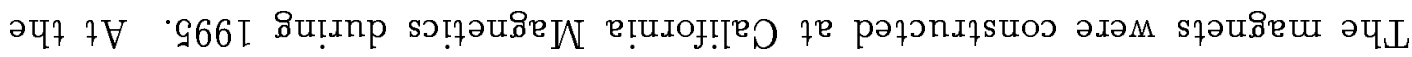

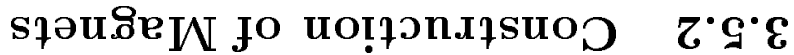

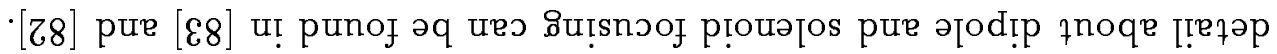

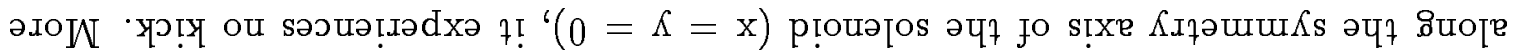

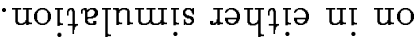

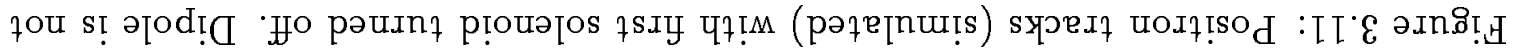

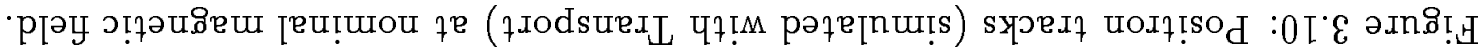
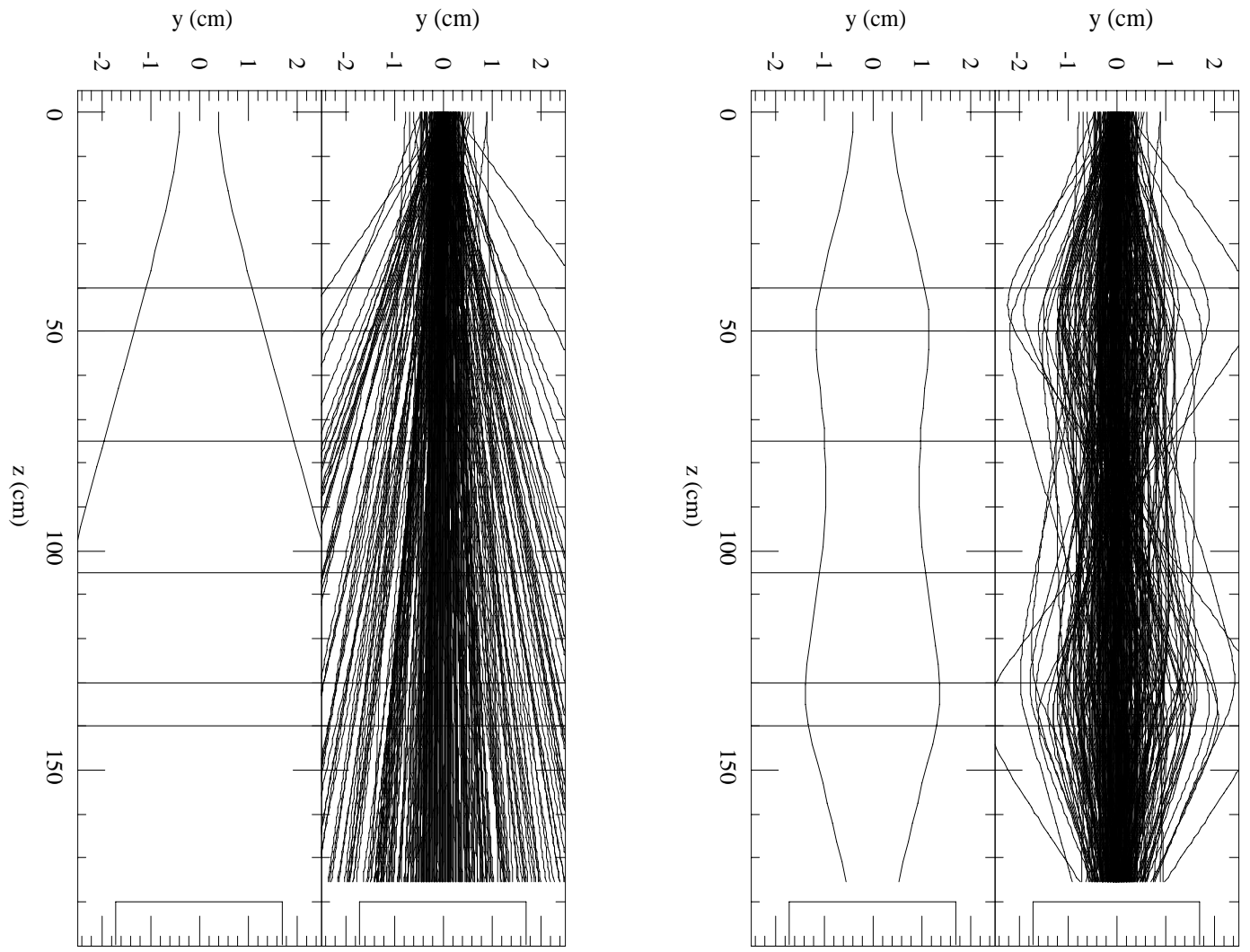

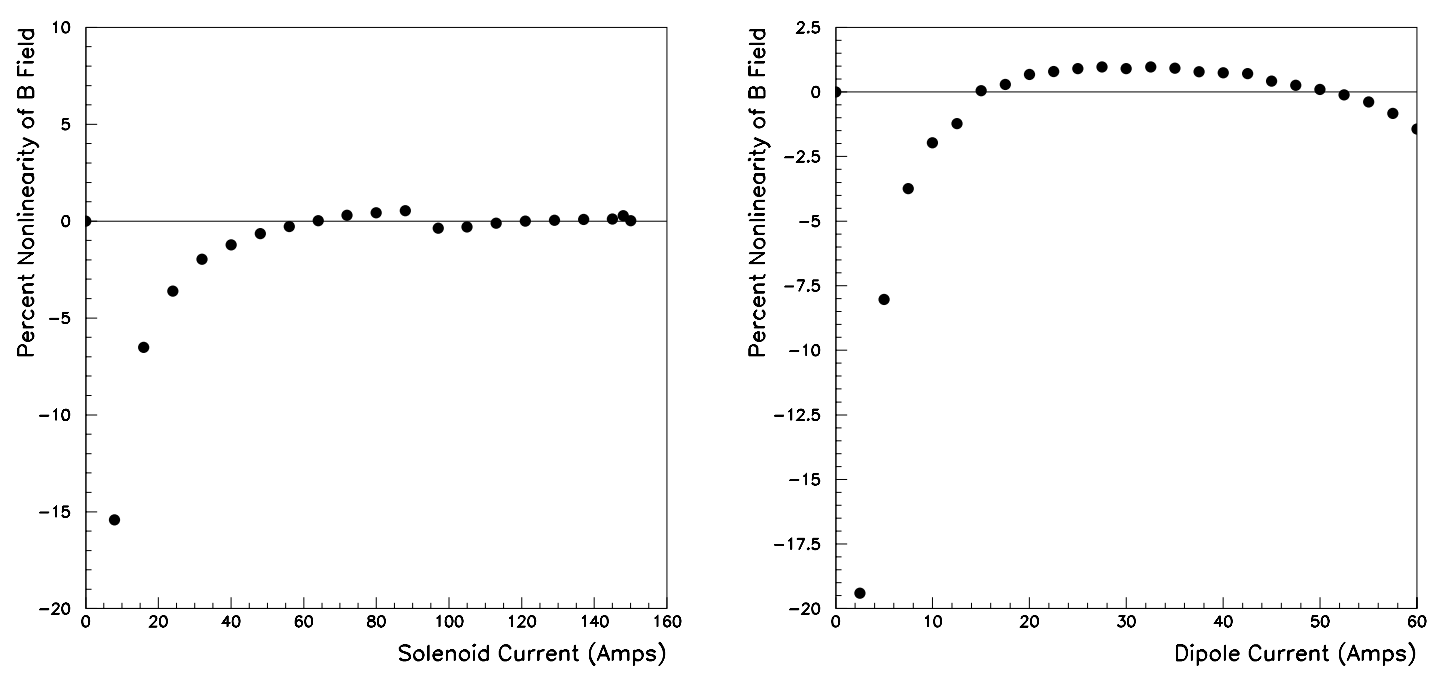

Figure 3.12: Nonlinearity of solenoid, dipole magnetic fields with applied current. The fields vs currents for different currents were fit to a straight line. The difference of the measured magnetic fields from the fit are displayed as percentages. The dependence is nearly linear at higher current but exhibits some deviation at low currents.

winding, a thin layer each of (2 mil) of Kapton Polyamide film, "B" Stage epoxy-filled tape, Nomex Type 410 Class R tape, and P-21 black tape was glued over the cables for protection from the oxidation, etc.

\subsubsection{Magnetic Field Properties}

When the magnets arrived at Fermilab, initial testing was done. The solenoids perform linearly throughout their range of current up to $160 \mathrm{Amps}$. The dipole was found to have a significant (3\%) hysteresis effect when the polarity of the current (and hence B-field) was switched. In order to track the dipole's B-field, a Hall probe was placed inside the dipole. The dipole has to be cycled a few times in order to bring the magnets to a reproducible field setting within $0.5 \%$. It was originally thought that the solenoids would not need their polarity switched to observe electrons while the dipole needed to be field reversed. 
The Hall probe is a solid state detector, $1 " \times 1 "$ in area. The probe is glued to the bottom pole of the dipole magnet and held down by an aluminum bracket to keep it normal to the field and prevent the cables from applying torque to the probe. Temperature compensation is included at the detector. The analog level is sent to the counting room over 30 feet of cable and digitized. The device measures the magnetic field to a precision of 0.01 gauss, roughly $10^{-4}$ of the field value. However, the resolution is limited to \pm 0.5 gauss. When the magnets were turned off and/or were field reversed, there is a remnant field measured at less than 10 gauss. The dipole was always set by adjusting the current to get the correct field.

\subsubsection{Beam Test Apparatus}

\section{- Table Construction}

A non-magnetic (aluminum) table was constructed to support the spectrometer elements. The table height was such that the beampipes were at the same height as the beam (28.5"). The top part of the table was a $1 / 2$ " plate attached to girders. This slid on Teflon pads (for easy horizontal positioning) attached to the bottom part, which was a 3 point truss to allow easy vertical positioning. Screw adjustments provided easy translational and rotational motion of the table in the horizontal plane to submillimeter precision.

- Alignment Blocks raised the elements such that the vertical positions of the center of the vacuum pipe, the source point, and the center of the magnets lined up. After testing, the solenoids had to be repositioned to move the beam back to the center of the beampipe when it was found to be partially hitting the wall. Moving the solenoids by equal distances in the same or opposite direction in the transverse plane allowed changing the beam position in those directions independently for the two different solenoid tunes. 
- Vacuum After installation the vacuum was brought down to $10^{-5}$ Torr with a roughing pump and turbomolecular pump. The system was leak checked with a standard Helium leak (fixed leak rate). The vacuum pipe was tested down to $10^{-6}$ Torr with a leak rate of $4 \times 10^{-8} \mathrm{cc} / \mathrm{sec}$. When attached to the accumulator vacuum in the AP50 tunnel, the vacuum reached $10^{-7}$ Torr.

- Water cooling Since the maximum solenoid current generated substantial heat $(1 \mathrm{~kW})$, two water cooling circuits were designed into each of the 3 magnets (dipole had 1 per pole). During electron source testing, a deionized water system was setup, (see figure 3.13). The temperature was kept constant at $80^{\circ} \mathrm{F}$ with a small flow rate $(\max 20 \mathrm{gpm}$ at $120 \mathrm{psi}$ ). In operation, each magnet circuit took less than $1 \mathrm{gpm}$ with 50 psi pressure drop. Two trip circuits (bimetallic switches) were attached to each of the magnets so that if the coil temperature rose above a threshold, the appropriate power supply would be turned off. In the tunnel, the cooling came from the Low Conductivity Water (LCW) water circuits already cooling the large ring magnets. The trip circuitry was the same.

\subsubsection{Monoenergetic Electron Source Testing}

To test the focusing at one energy, a bismuth-207 $\left({ }^{207} \mathrm{Bi}\right)$ radioactive source with an activity of $10 \mu \mathrm{Ci}\left(3.7 \times 10^{5} \mathrm{~Hz}\right)$ was used. The source is deposited on a 1 " diameter nickel substrate. An aluminum holder held the disk and was attached to the target wheel. The ${ }^{207} \mathrm{Bi}$ source was actually about $1 \mathrm{~mm}$ in size and covered with a coating which slightly affects the energy and the angle of the $e^{-}$as it leaves the source. The fact that the line energies [13], $E_{e^{-}}=0.975 \mathrm{MeV}, 1.07 \mathrm{MeV}$, were well below the range expected in the antihydrogen data, $2<E_{e^{+}}<4.5 \mathrm{MeV}$, meant that using the test data would require some assumptions about how the focusing would scale with 
E-862 De ionizing and Filter System ByRED5/25/95 Load on System 1KW 1-2 GPM Rev. 19/14/95

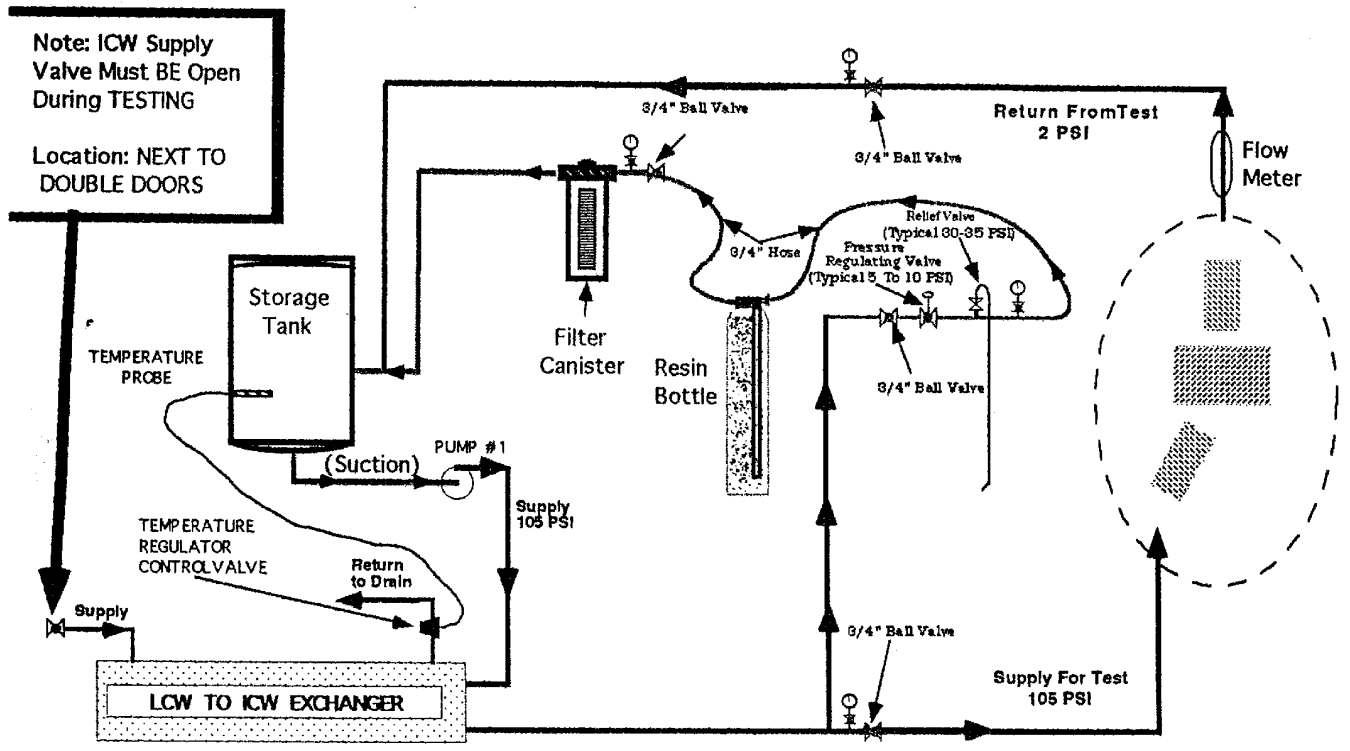

Figure 3.13: Deionized water system in Lab 6.

the energies. The two lines are $7 \%$ and $2 \%$ of the rate respectively [13]. Still, the need to accurately image a beam spot at any energy and the ability to resolve the two closely spaced peaks made the test data important. The beampipe at the center of the solenoid was $1.5 "$ in radius. This meant the fractional acceptance was about $3.6 \times 10^{-3}$ and the expected rate was $220 \mathrm{~Hz}$. The best rates measured were about $50 \mathrm{~Hz}$ with no collimation.

Table 3.3: Magnetic field settings vs. beam energy

\begin{tabular}{|c||c|c|c|c|c|}
\hline $\begin{array}{c}p_{\bar{p}} \\
(\mathrm{GeV} / \mathrm{c})\end{array}$ & $\begin{array}{c}E_{c m, \bar{p} p} \\
(\mathrm{MeV})\end{array}$ & $\begin{array}{c}p_{e^{+}} \\
(\mathrm{MeV} / \mathrm{c})\end{array}$ & $\begin{array}{c}B_{\text {dip }} \\
(\text { Gauss })\end{array}$ & $\begin{array}{c}B_{\text {sol, } 1 \text { st }} \\
\text { (Gauss })\end{array}$ & $\begin{array}{c}B_{\text {sol,2nd }} \\
\text { (Gauss })\end{array}$ \\
\hline 2.562 & & 1.395 & 135 & 288 & 461 \\
3.71 & 2.99 & 2.021 & 195 & 417 & 667 \\
4.068 & 3.097 & 2.216 & 214 & 457 & 731 \\
5.615 & 3.527 & 3.058 & 296 & 631 & 1009 \\
5.97 & 3.619 & 3.25 & 314 & 671 & 1073 \\
6.233 & 3.686 & 3.395 & 328 & 701 & 1121 \\
8.9 & 4.30 & 4.847 & 469 & 1000 & 1600 \\
\hline
\end{tabular}




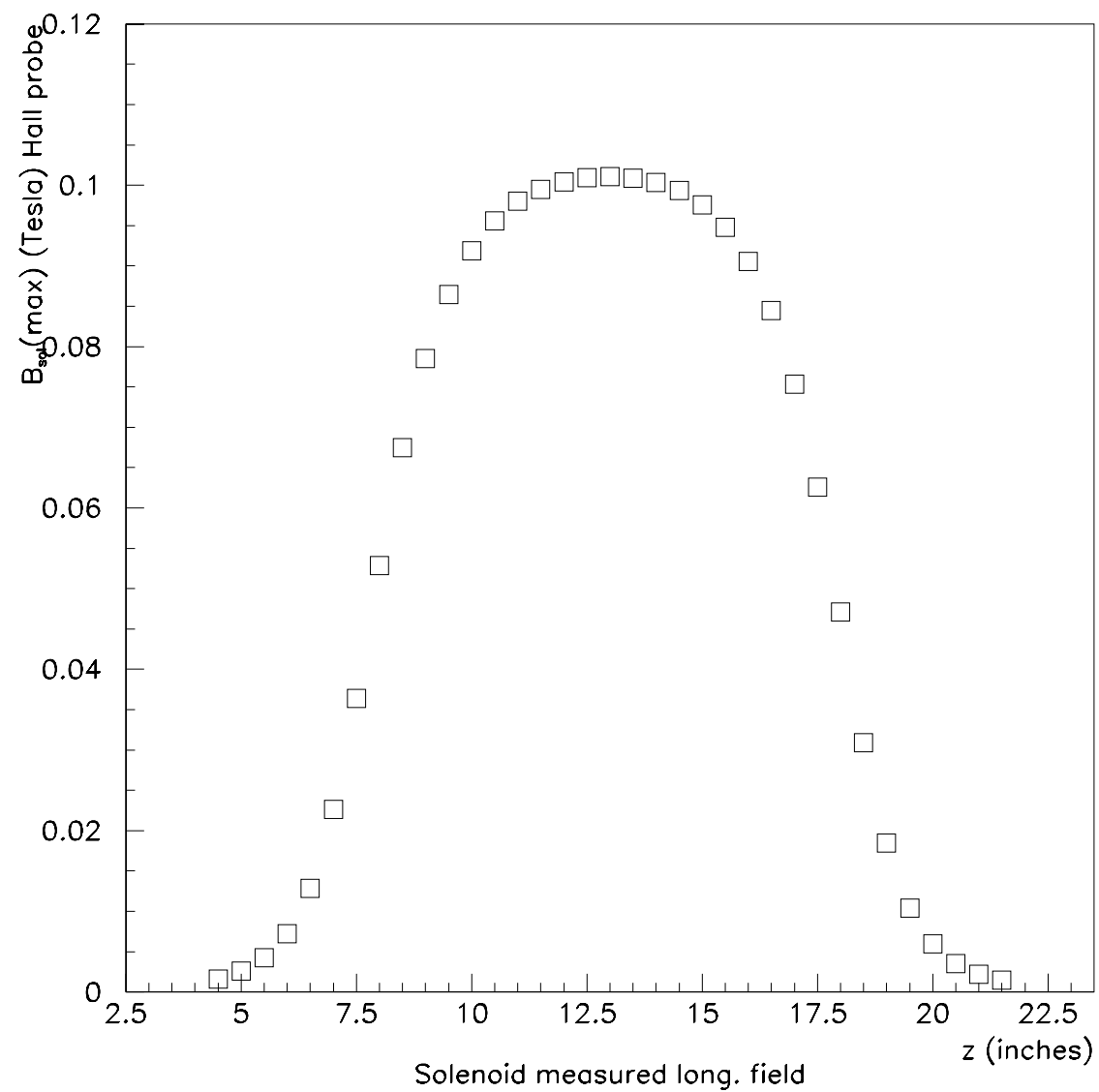

Figure 3.14: Measured magnetic field of solenoid along axis. 

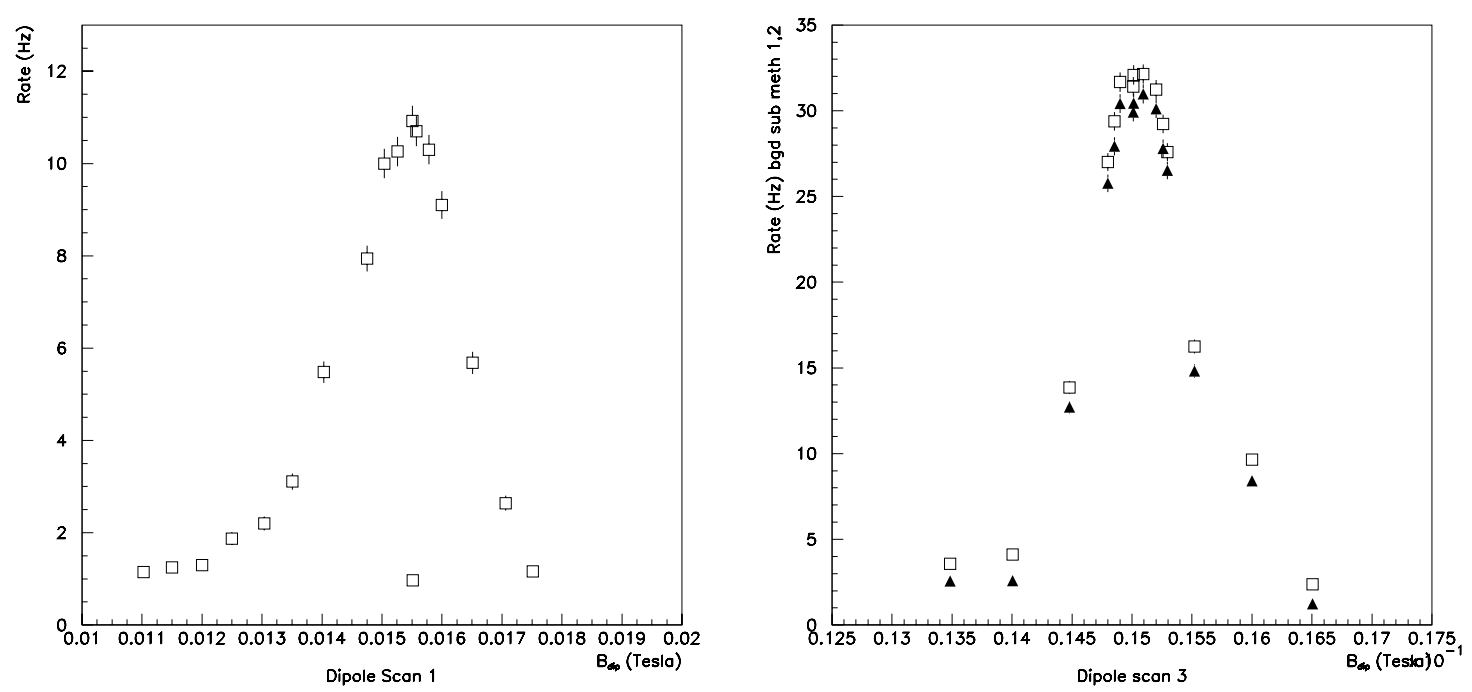

Figure 3.15: Testing the spectrometer with the bismuth source in Lab 6. Count rate scans over the dipole applied current.
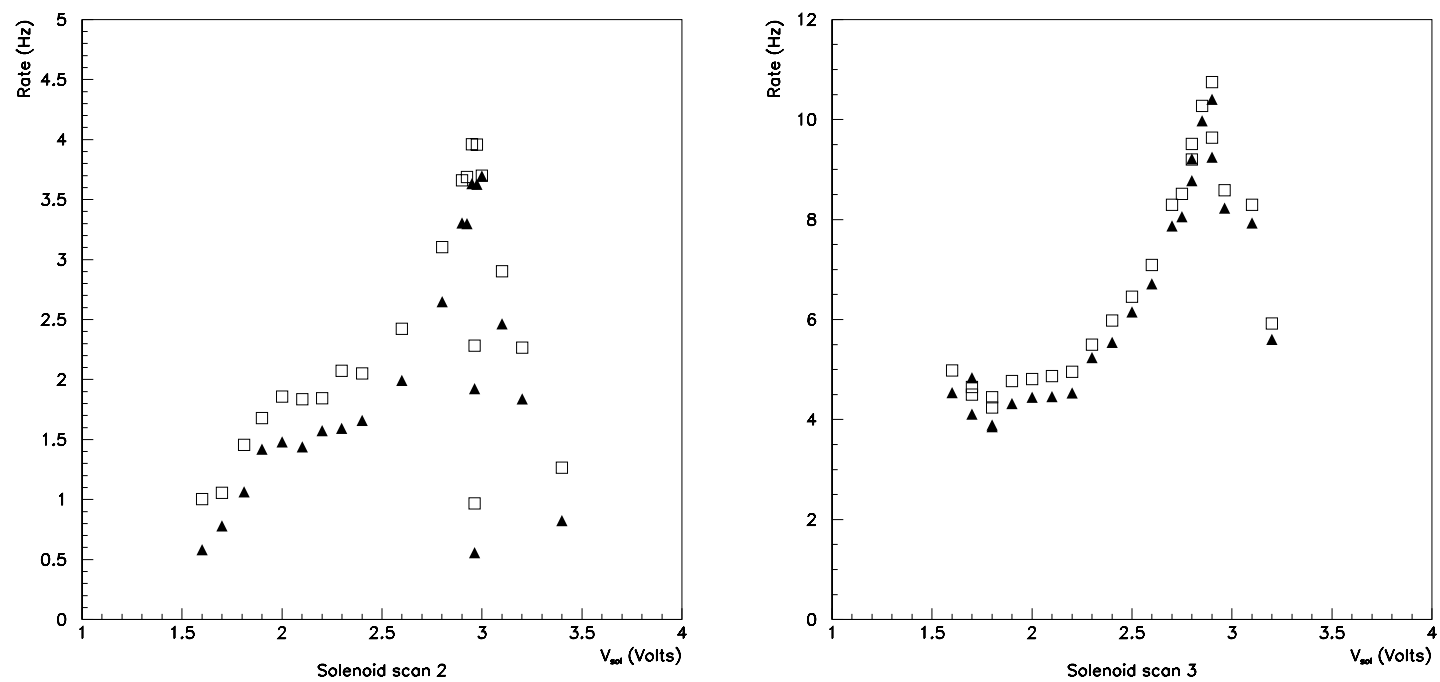

Figure 3.16: Testing the spectrometer with the bismuth source in Lab 6. Count rate scans over the solenoid applied current. 
To detect all the electrons possible at the end, a $2 " \times 2 " \times 1 / 2 "$ plastic scintillator square was directly mounted to a phototube and brass apertures used to collimate the beam selectively at either end. The signals for electrons with the phototube set at $-1800 \mathrm{~V}$ were about $500 \mathrm{mV}$ maximum amplitude and could be distinguished clearly from gamma ray hits and MIP hits with much higher (2-3 V) amplitudes. The electron signal rate changed consistently when the acceptance of the spectrometer was changed. The signals were discriminated at two different thresholds of $300 \mathrm{mV}$ and $1 \mathrm{~V}$ and counted. The rates were compared, the latter being used as the background rate since most background was from high energy particles. The background rate was checked by placing a $>1 / 4$ " aluminum plate $6 " \times 6$ " before the scintillator and measuring the rate. This background rate was subtracted from the electron hit rate.

Shown in figure 3.14 is the measured $B_{z}$ profile along $z$ in the solenoid. The plot demonstrates the symmetry of the field along the central symmetry, $z$, axis of the solenoid. The next two figures, (3.15, and 3.16) are the results of scanning the DC currents of the magnets over a certain range corresponding to the bismuth source energy. Figures 3.17 , and 3.18 were scans nearly identical to the previous two but done after final alignment in the tunnel. The solenoid is set to a value found by simulation to give good focusing for $0.975 \mathrm{MeV}$ kinetic energy electrons and the dipole scanned over a $20 \%$ range in current. The peak corresponding to the 0.975 $\mathrm{MeV}$ electron line is visible. The smaller bump at the right corresponds to the electron line at $1.047 \mathrm{MeV}$. The dipole current was held fixed at the peak and the solenoid current ramped to investigate focusing. Although the magnetic field needed to focus is linearly dependent on applied current, it is not as easy to predict the effect of changing the solenoid currents as it is for changing the bend angle. The large peak to the right corresponds to the low dispersion solution where the electrons cross the central trajectory at the symmetry point in the dipole. This solution would obviously 
have better collection efficiency. A peak for the high dispersion solution is harder to see at lower solenoid current. 


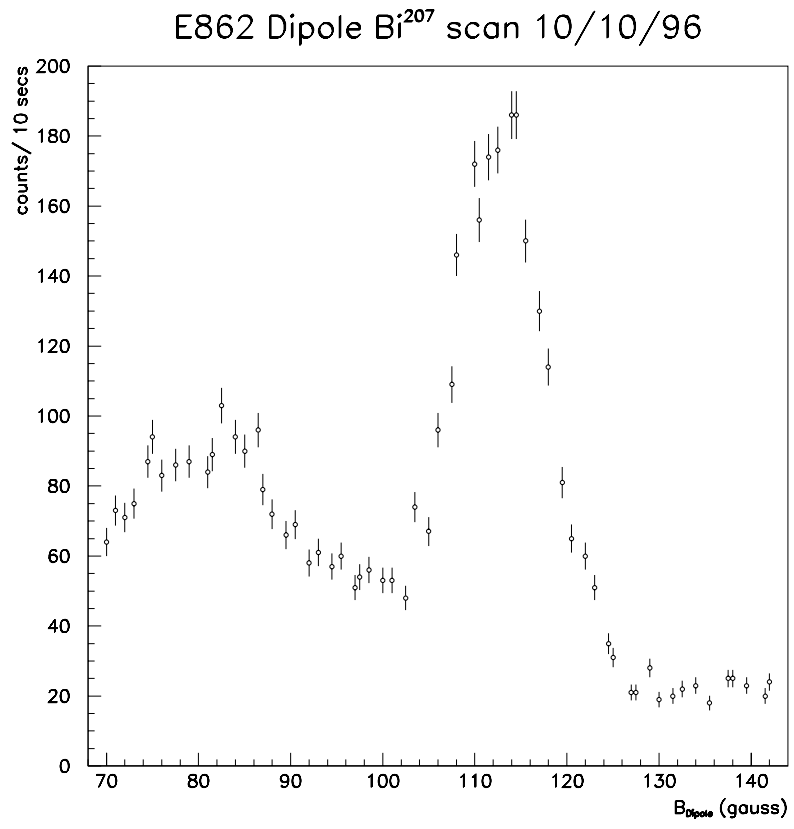

Figure 3.17: Scan over the dipole field for ${ }^{207} \mathrm{Bi}$ after final setup in tunnel.

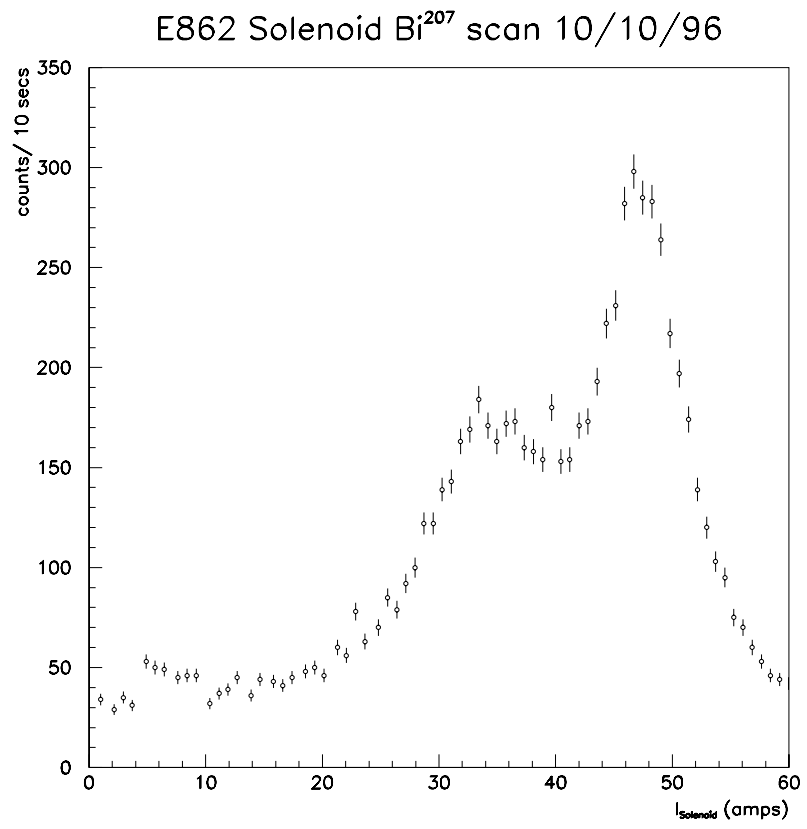

Figure 3.18: Scan over the solenoid current for ${ }^{207} \mathrm{Bi}$ after final setup in tunnel. 
Plot 3.19 shows the effect on the electron rate of moving the source out of the plane of the beamline by rotating the wheel. The source position dependence gives a feel for the acceptance of the system. Specifically, scans were done with the electron line source to see how far away the beam could be without missing the positron spectrometer. The limiting factor seems to be the size of the T-shaped beampipe section leading up to the $\mathrm{e}^{+}$scintillator (1") as projected back to the source plane. This projection is not the same for the two tunes. As seen in figure 3.19, the distribution for the high dispersion tune shows an offset of about $4.5 \mathrm{~mm}$ above the focusing plane whereas the low dispersion tune does not show any noticeable offset. During alignment studies, the first solenoid was offset by $3 / 16$ " vertically in order to center the beam on the scintillator which lies in the image plane. Because of symmetry, the second solenoid was moved $-3 / 16^{\prime \prime}$. Thus, it was thought the electrons might be seeing a magnetic aperture (a significant deviation of the field from its required value for this momentum) although the solenoid has a nearly constant field across most of its inner diameter.

The spectrometer has a slightly different focusing plane for the two tunes. The high dispersion tune focuses positrons in a plane rotated about $45^{\circ}$ with respect to the horizontal so that the offset in the object plane results as evidenced in figure 3.19. The object plane is the plane containing the source.

\subsection{Positron Detectors}

The purpose of the positron counter, a plastic scintillator, was to detect the positron, measure its arrival time precisely enough to trigger, and insure its annihilation with an electron. The annihilation yielded back-to-back gamma rays which are detected by sodium iodide, $\mathrm{NaI}(\mathrm{Tl})$ cylinder halves, (see figure 3.21 ). The gamma 

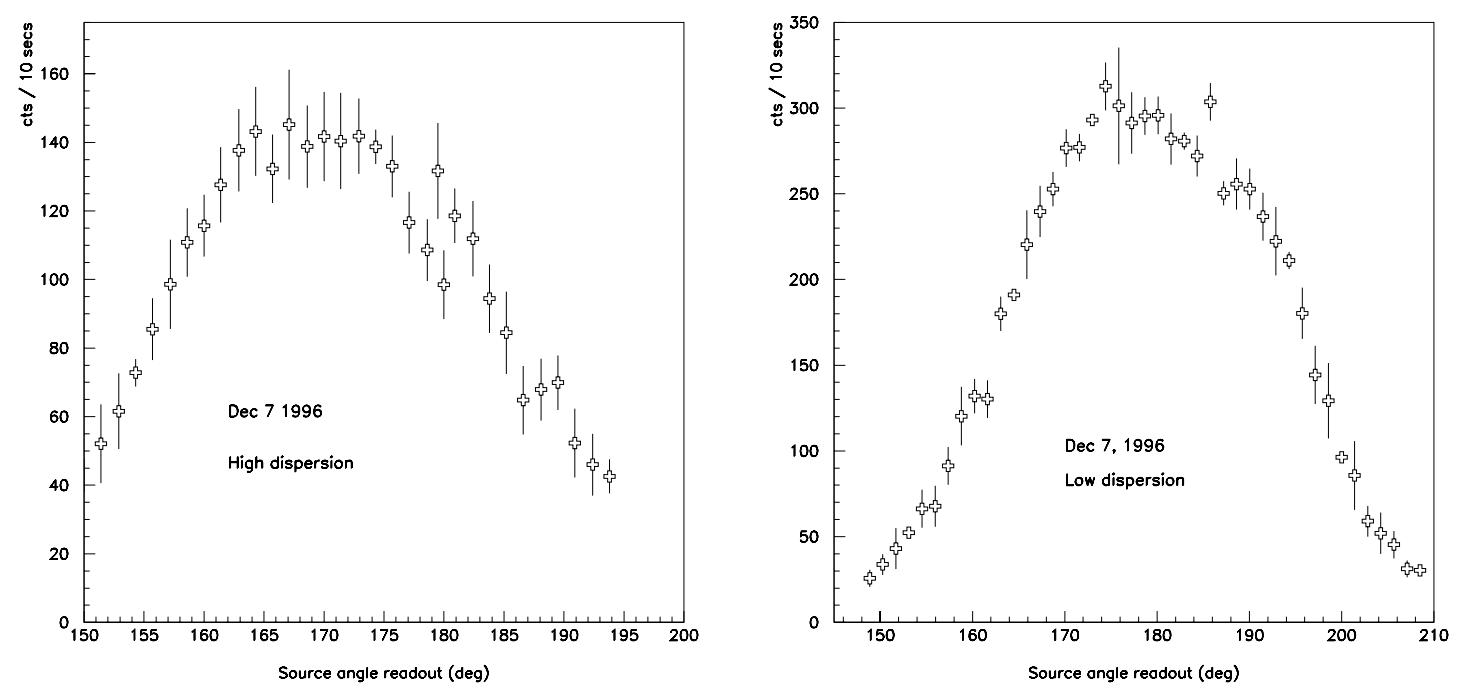

Figure 3.19: Vertical position scan, for high and low dispersion tunes

ray energy and arrival time as well as positron energy (from the plastic scintillator) were measured as well.

\subsubsection{Positron Counter}

A plastic scintillator plug $1 "$ in diameter and 1 "thick served as the end plug for the high-vacuum as well as stopping the $e^{+}$and annihilating it. The half inside the beam pipe was actually only 0.9 " in diameter. When a positron enters the scintillator, one of several things can happen. It can back scatter. It can lose all its energy to the scintillator through ionizations and annihilate before exiting, which it will do $99 \%$ of the time if it goes straight into the scintillator. It can cut the corner of the scintillator and annihilate in the sodium iodide or the aluminum inner case. Finally, if it is close to the edge, it can be scattered into the sodium iodide as opposed to having a straight path crossing into it; in this case, it most likely would annihilate in the aluminum 1 " tube. The first possibility is small and the probability depends on the energy of the positron. Monte Carlo calculations using EGS and GEANT showed the fraction of 
Table 3.4: Probability $\mathrm{e}^{+}$will back scatter from the plastic scintillator

\begin{tabular}{|c|c|}
\hline $\mathrm{e}^{+}$Energy & Probability \\
\hline $2 \mathrm{MeV}$ & $0.011(90 \%)$ \\
$3.5 \mathrm{MeV}$ & $0.008(90 \%)$ \\
\hline
\end{tabular}

the time it is back-scattered completely out of the scintillator (see table 3.6.1). The remaining possibilities, though, depend on how collimated, focused, and centered the "beam" of positrons is. If the positron annihilates outside the plastic scintillator, the gamma rays can both deposit energy in the one half. If one is scattered, it can leave part of its energy in one half and part in the other half. We have no way of resolving these annihilations by observing the two photon opening angle. The energy deposit in each half may fall above the $511 \mathrm{MeV} \pm \delta E_{\gamma}$ energy window. The total energy should be $1022 \mathrm{MeV}$, though, unless the $e^{+}$annihilates in flight.

Most positrons come to rest before annihilating. However, the positron can annihilate while in flight, which alters the gamma ray energies. The cross section per electron as a function of $\gamma=E_{e^{+}} / m_{e}$ is [84]:

$$
\sigma_{a n n i h}=\pi r_{o}^{2} \frac{1}{\gamma+1}\left[\frac{\gamma^{2}+4 \gamma+1}{\gamma^{2}-1} \ln \left(\gamma+\sqrt{\gamma^{2}-1}\right)-\frac{\gamma+3}{\sqrt{\gamma^{2}-1}}\right]
$$

The probability of annihilating in flight at a certain energy can be calculated by dividing the cross section for annihilation, which is a function of energy, by the energy loss per unit distance for that material [85],[86] and then integrating up to the incoming energy. The material density is denoted as $\mathrm{N}$ (atoms $/ \mathrm{cm}^{3}$ ). The energy loss per distance ionizations for high energy electrons is $[85]\left(\mathrm{I} \sim 15 \mathrm{eV}, r_{o}=2.8 \times 10^{-15}\right.$ $\mathrm{m)}$ :

$$
-\frac{d E}{d x}=N Z 2 \pi r_{o}^{2} m_{e}\left(\frac{E}{\sqrt{E^{2}-m_{e}^{2}}}\right)^{2}\left[\ln \left(\left(E-m_{e}\right)\left(E^{2}-m_{e}^{2}\right) / 2 m_{e} I^{2} Z^{2}\right)+\left(\frac{m_{e}}{E}\right)^{2}\right]
$$


If the material is thick enough to stop the positrons, the probability of annihilation in flight per $e^{+}$is, (also shown in figure 3.20 ):

$$
P_{\text {in-flight }}=-Z N \int_{m_{e}}^{E_{i}} \frac{\sigma(E) d E}{d E / d x}
$$

Otherwise, the lower limit of integration is replaced by a cutoff energy $E_{c}$ which depends on the thickness and the probability of annihilating at rest is not equal to 1 ,

$$
P_{a t-r e s t}=\exp \left(-\left.Z N\left(\frac{\sigma}{d E / d x}\right)\right|_{E_{c}}\right)
$$

The annihilation cross section has a local maximum at $511 \mathrm{keV}$ kinetic energy ( $\mathrm{E}=1$ $\mathrm{MeV}$ ) and then drops off at higher energies. The percentage of annihilation in flight is between 5 and $10 \%$ for plastic scintillator. The effective $\mathrm{Z}$ for polyvinyltolulene used in plastic scintillator (monomer $2-\mathrm{CH}_{3} \mathrm{C}_{6} \mathrm{H}_{4} \mathrm{CH}=\mathrm{CH}_{2}$ ) is $\mathrm{Z}=1.1$.

The scintillator was coupled with optic epoxy directly to a 1" photomultiplier tube since the hole in the NaI was only $11 / 2$ " in diameter. The phototube was chosen for speed as well as size, having an intrinsic risetime of $2 \mathrm{nsec}$, since it is an intrinsic part of the trigger. The base had 10 stages. The spread of the time of arrival of the pulse for a particular positron energy was about 500 psec. Of course, the trigger timing was less crucial than the time of flight measurement, and the transit time spread in the focusing system was considerable. The scintillator pulse was typically $20 \mathrm{nsec}$ wide. It was split, discriminated at the $-100 \mathrm{mV}$ level and the logic pulse digitized in 409625 psec bins. The analog pulse was digitized in a charge integrating $\mathrm{ADC}$ in $20480.25 \mathrm{pC}$ bins using a gate width of $200 \mathrm{nsec}$.

\subsubsection{Sodium Iodide(Tl) Gamma Ray Detector}

After the $e^{+}$loses most if not all of its kinetic energy in the scintillator, it annihilates and two essentially back to back photons are detected by the two halves 

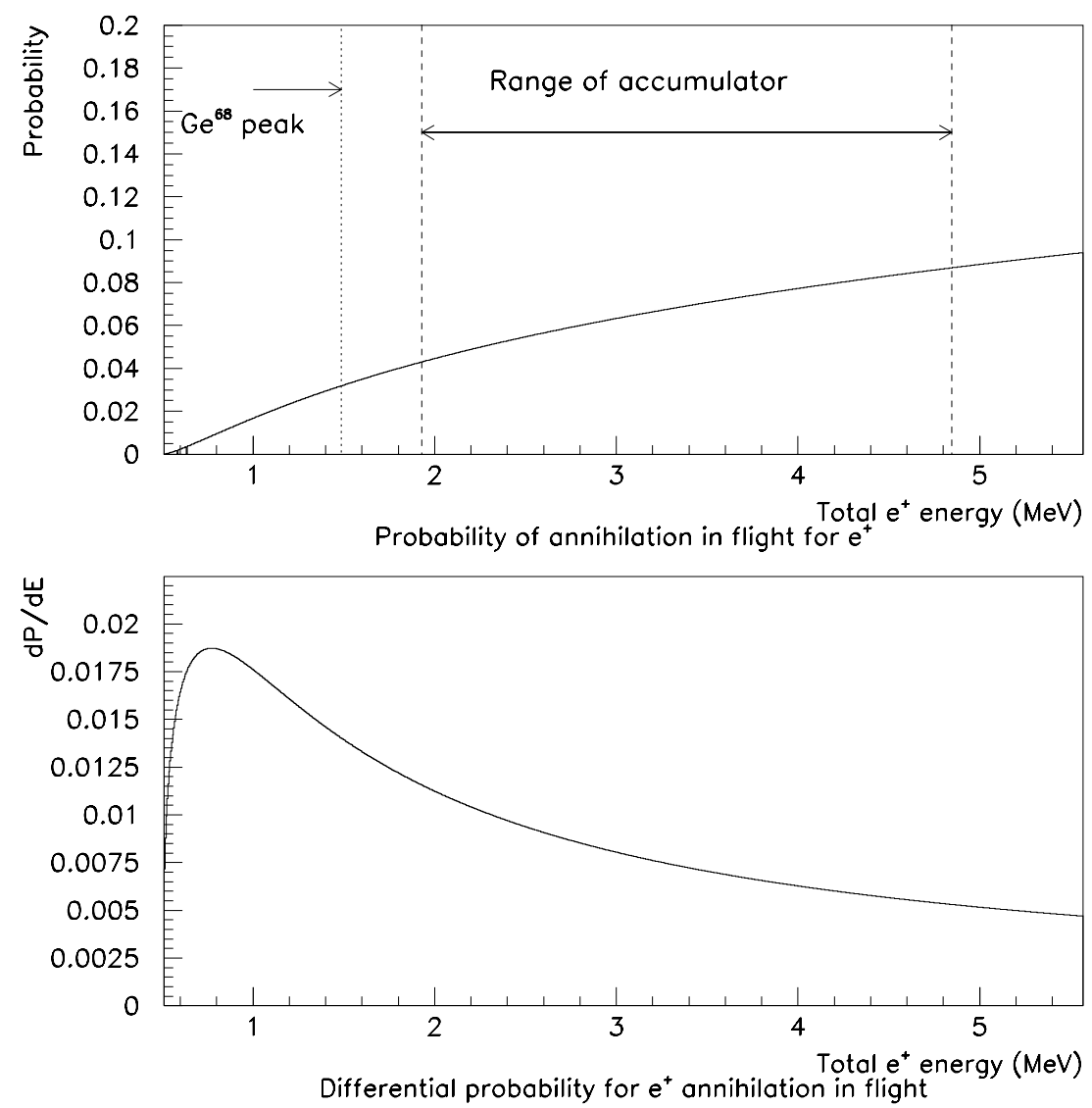

Figure 3.20: Probability for an $e^{+}$annihilation in flight as a function of $e^{+}$total energy. The peak at $1.022 \mathrm{MeV}$ total energy is clearly shown in the differential probability distribution. 
of a sodium iodide cylinder $7 "$ in diameter surrounding the scintillator. The sodium iodide, being hygroscopic, is completely contained within an aluminum can $1 / 16^{\prime \prime}$ thick with 6 holes on the top side for phototubes glued directly to the NaI. The bore in the middle is lined with aluminum as well and this bore represents a loss of acceptance for a single photon of $1.86 \%$. The aluminum lining of the flat faces separating the halves represents a loss of geometrical acceptance of $1.14 \%$ again for a single photon. When the annihilation gammas enter the sodium iodide, they get Compton scattered by electrons. Thus the energy window had to be wide enough to accept hits in which one of the annihilation gammas Compton scattered.

The phototubes and bases were adjusted for overall gain matching of each triplet. This facilitates summing the analog pulses and reading out only 2 analog signals but retains the safety factor of allowing one to fail while still recording $70 \%$ of the half's energy on average. A looser cut on photon energy would still reject events not having 2 hits. The analog pulses after 550 nsec of cable were read into a $1 \mu$ sec gate width charge-integrating ADC. Typical energy resolution expected of sodium iodide is 8$10 \%$ for photon energies on the order of $1 \mathrm{MeV}[87]$. A copy of the analog signal was amplified by $10 \mathrm{X}$ and discriminated at $-400 \mathrm{mV}$ corresponding to about $250 \mathrm{keV}$ photons. The signal's arrival time was digitized in $204850 \mathrm{psec}$ bins.

The high voltages were set as high as possible on the plateau curves, (see figure 3.22), without inducing a large amount of noise on the signals. The gains of the PMTs were adjusted at the factory to be equal, but the phototube bases were not adjusted. Each base had two screw adjusts, one for the focus and one strictly for the gain. Both were adjusted so all the signals were about equal (to $5 \%$ ) at the same applied voltage. The voltage level was then set at $1300 \mathrm{~V}$. The operating range is 900 to $1400 \mathrm{~V}$ but the efficiency is very low below $1300 \mathrm{~V}$ because of high threshold. 

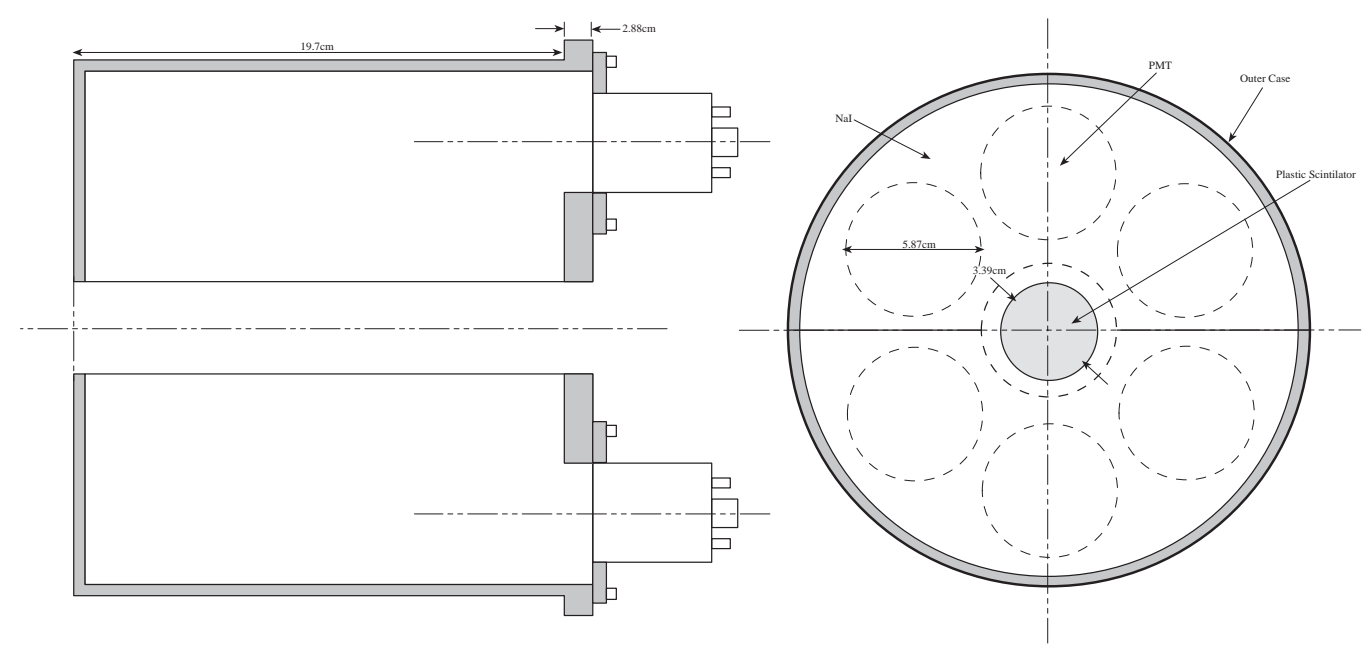

Figure 3.21: Diagram of sodium iodide detector

The pileup makes the signals very noisy above $1350 \mathrm{~V}$. The source used for testing, $\left(C s^{137}\right)$, had a higher rate than the expected data rate for beam running $(>1 \mathrm{kHz})$.

\subsubsection{Positron Source Testing}

The ${ }^{68} \mathrm{Ge}$ source has a positron beta spectrum, (see figure 3.23 ), as well as a discrete spectrum of gamma rays resulting from nuclear transitions. This source was used to verify that the $\mathrm{e}^{+}$counter signals were timed relative to the $\mathrm{NaI}(\mathrm{Tl})$ detector and to see that the gain of the $\mathrm{NaI}$ phototubes did not shift, by observing the position of the annihilation peaks. Typical fits of the ADC distributions to gaussian shapes (energy resolution determination) are shown in figure 3.24. As seen in figure 3.25, the $\mathrm{e}^{+}$ADC count depends on the current as the dipole and solenoid currents are changed together for the high dispersion tune. This was done to cut slices in momentum through the $\beta^{+}$spectrum. Figure 3.26 shows a similar dependence of the $\mathrm{e}^{+}$ADC value with the magnet current settings for the low dispersion tune. This tune accepts a large slice in momentum since the tracks are symmetric as they go through the 
Voltage Plateaus for E862 PMTs, 7/17/96
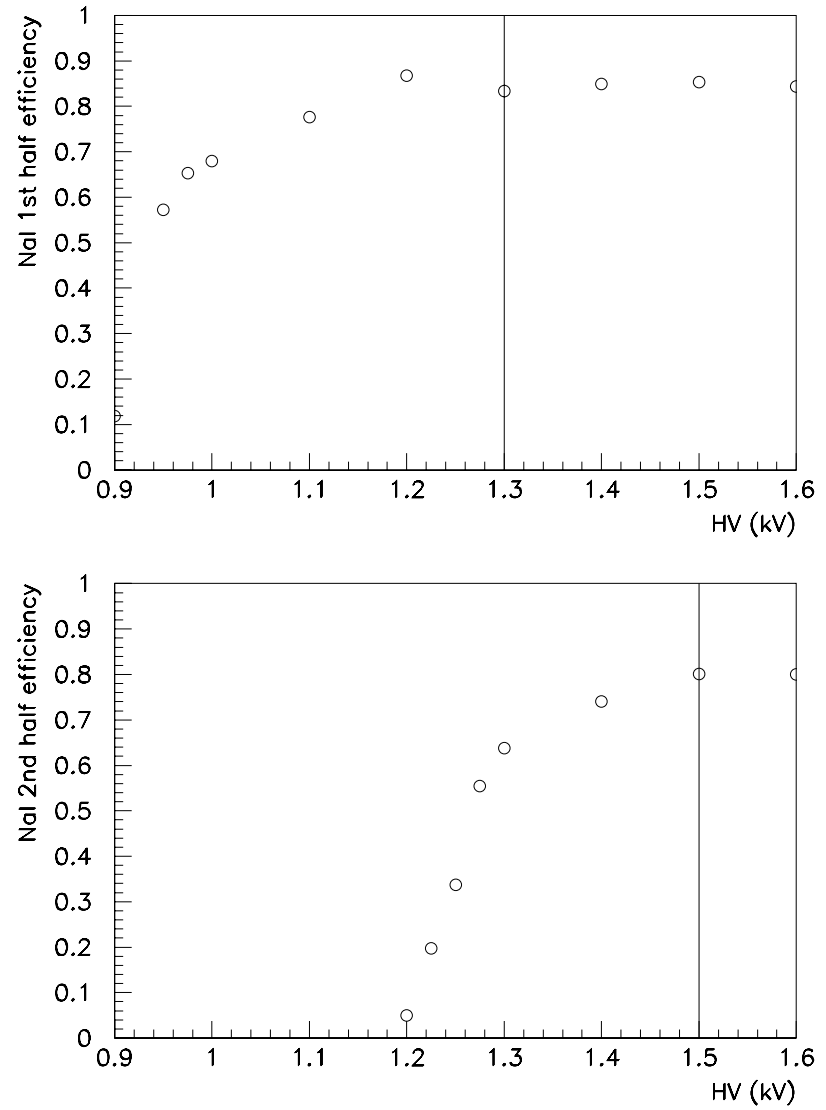

Figure 3.22: High voltage plateaus for the sodium iodide counters. 


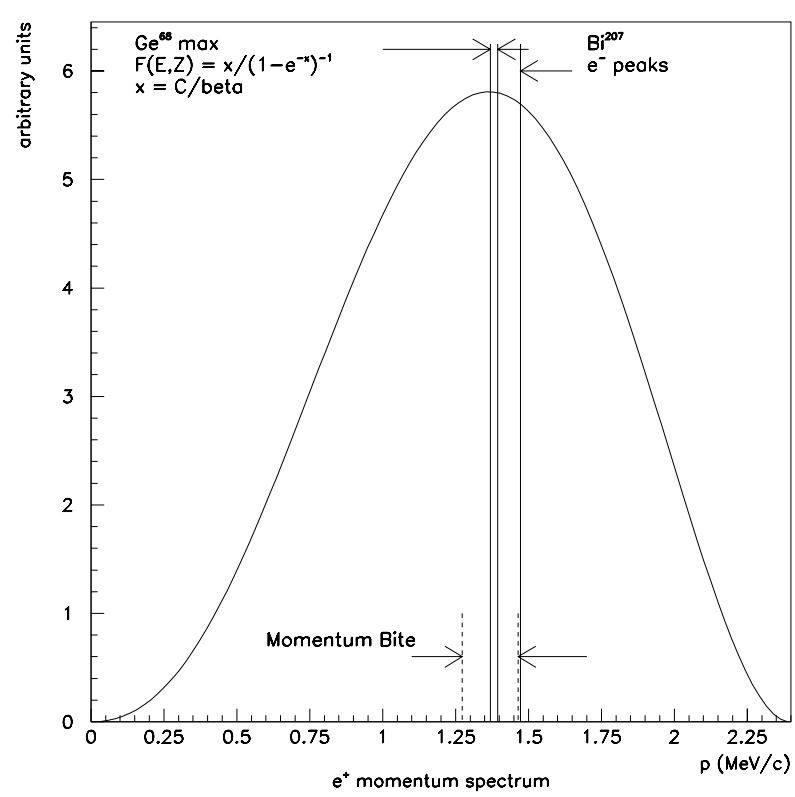

Figure 3.23: The $\beta^{+}$spectrum of ${ }^{68} \mathrm{Ge}$ showing a peak close to those of the bismuth source.

dipole. The annihilation gamma ray energy deposit remains constant as the magnet currents steer different energy positrons into the detector.

The widths of the adc peaks are plotted vs applied currents, (see figure 3.27), to show they are stable. Figure 3.29 shows the e+ ADC value versus dipole current for two different sets of points. The first is from November 1996 showing a lesser slope than the data from May 1997. The ${ }^{68} \mathrm{Ge} \beta^{+}$spectrum is superimposed to show where the e+ ADC values are expected to fall off at the end of the spectrum. The momentum range accepted here is $\pm 7 \%$. Without the solenoids, the acceptance would be $<4 \%$. There is a tight correlation between the momentum and energy deposit. One would expect the spectrum to have a steep slope, though, due to the assumption that the positrons stop in the scintillator and leave all their kinetic energy. It also may be due to the positron beam hitting the scintillator farther away from the center as the energy is increased. Another possibility is that the positrons simply do not stop in the scintillator but are annihilated in flight. 

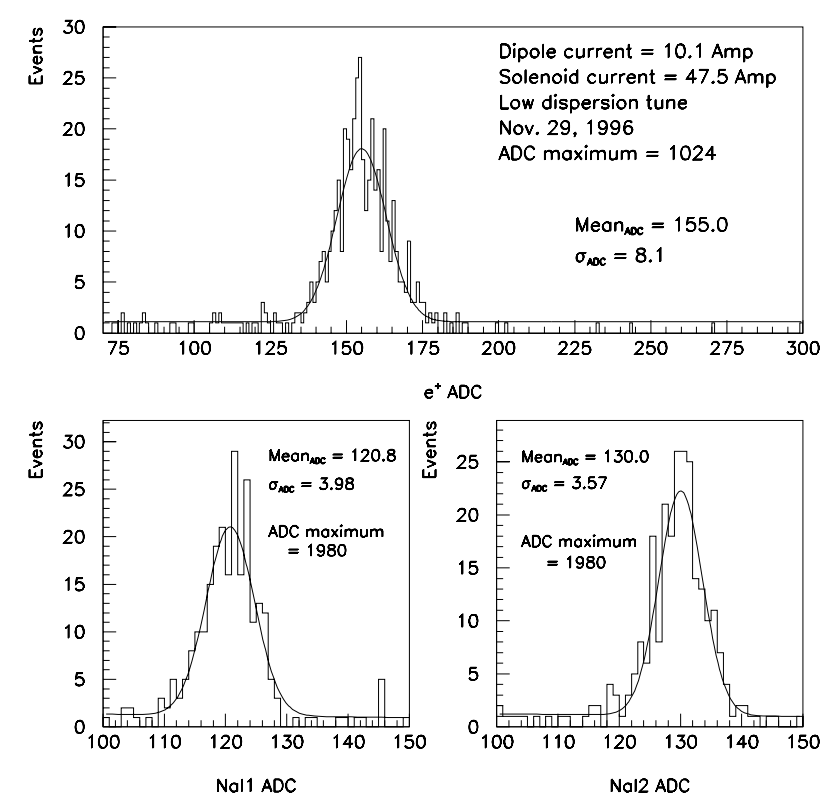

Figure 3.24: Gaussian fits to $\mathrm{e}^{+}$and $\mathrm{NaI}$ peaks.

The correlation of energy deposits in the NaI halves is shown in figure 3.28. Clearly a large number of events have at least one or both energies equal to $511 \mathrm{keV}$. One of the strange features of the $\mathrm{NaI}$ spectrums though is a significant fraction of deposits with energy much greater than the $511 \mathrm{keV}$ peak. One expects that some $511 \mathrm{keV}$ gamma rays will Compton scatter and indeed a low energy Compton edge is seen clearly in the test data. Roughly $5 \%$ of the data have either one or both $\mathrm{NaI} A D C$ values larger than the peak by $2 \sigma$. In the case of the data, there is some small amount of background from the accelerator environment. The calibration data in figure 3.28 clearly shows a band of events which have the sum of gamma energies equal to $2^{*} 511 \mathrm{keV}$ with one energy larger than $511 \mathrm{keV}$. This is possible since the positron can hit the plastic scintillator towards the edge, and annihilate inside one of the NaI halves instead. Both annihilation photons deposit their energy in that half. Even with an annihilation within the plastic, one of the gamma rays can Compton scatter out of one half backwards so that both photons arrive in the other half. 

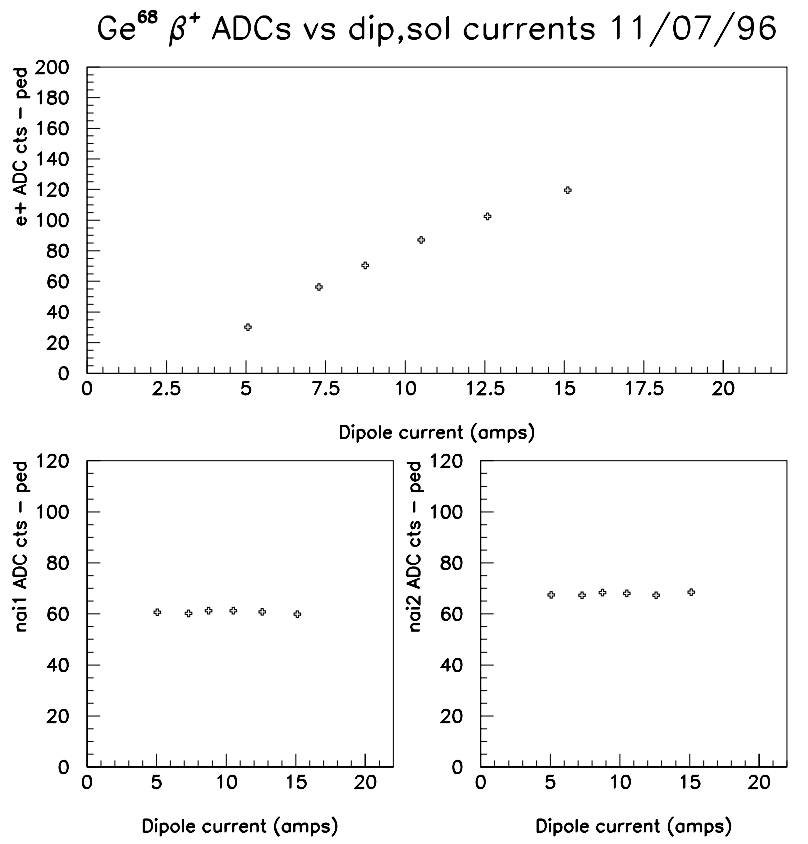

Figure 3.25: $\mathrm{e}^{+}$pulse height (ADC counts) versus dipole (and solenoid) currents for the high dispersion tune.
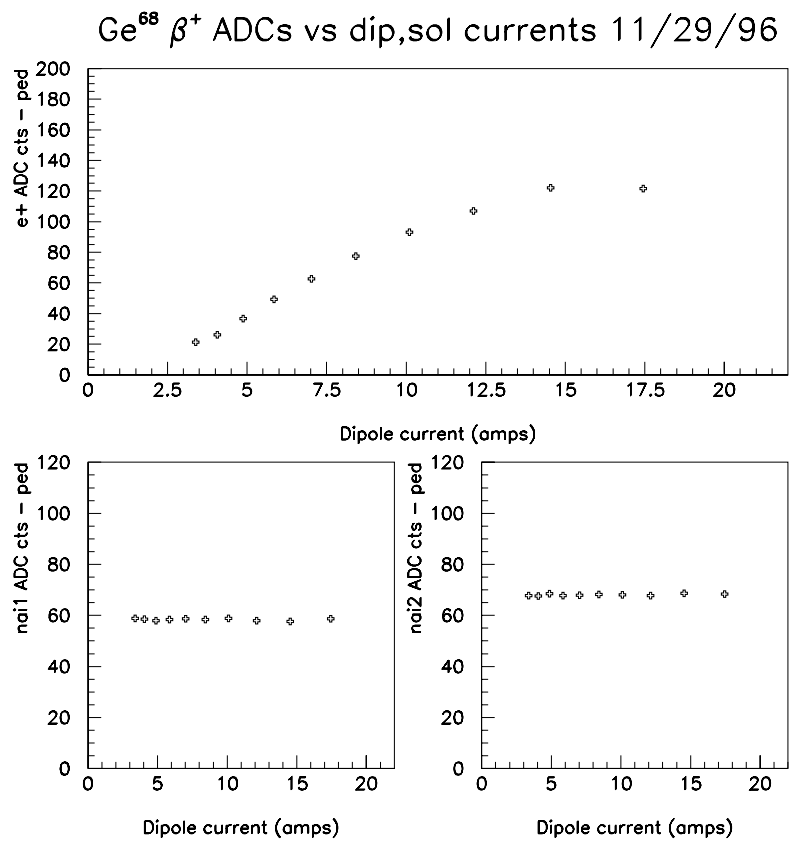

Figure 3.26: $\mathrm{e}^{+}$pulse height (ADC counts) versus dipole (and solenoid) currents for the low dispersion tune. 

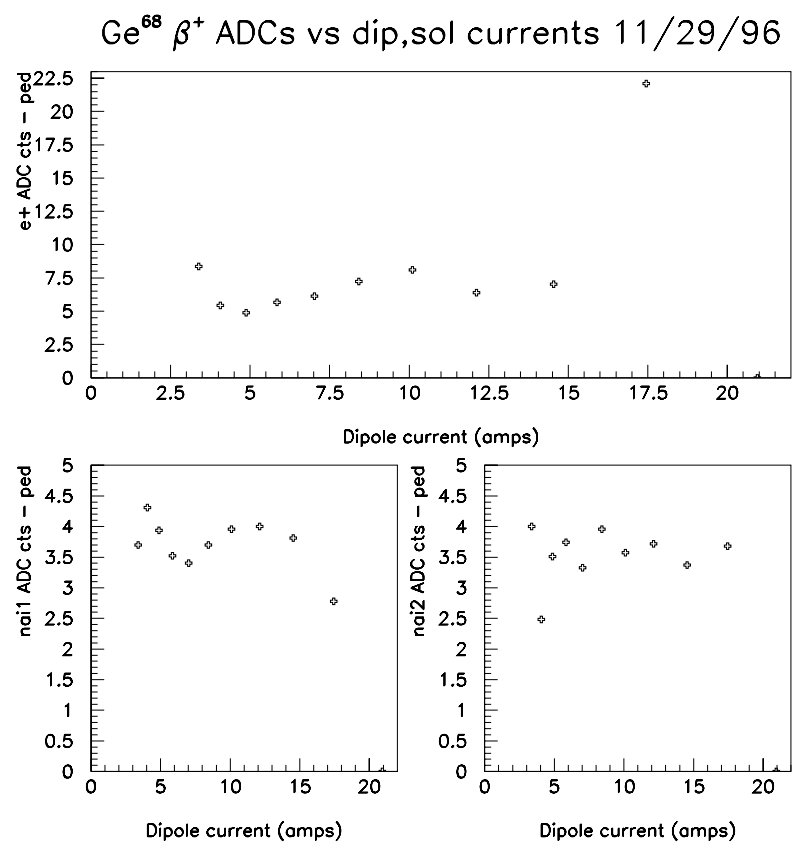

Figure 3.27: Widths of $\mathrm{e}^{+}$pulse height distributions' fit peaks as functions of applied magnet currents

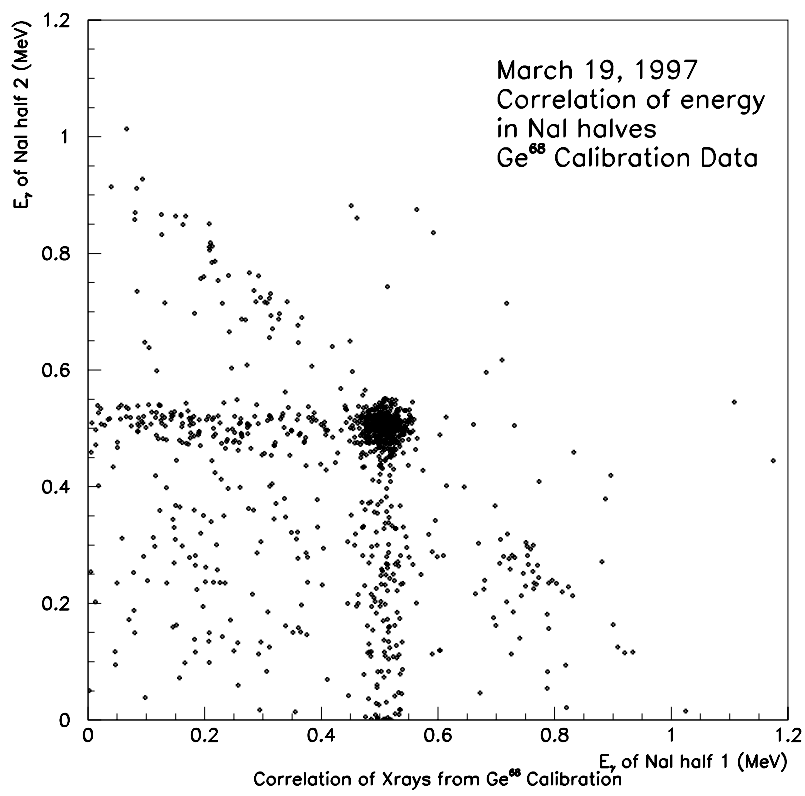

Figure 3.28: Correlation of $\mathrm{e}^{+}$annihilation ray energies in sodium iodide 


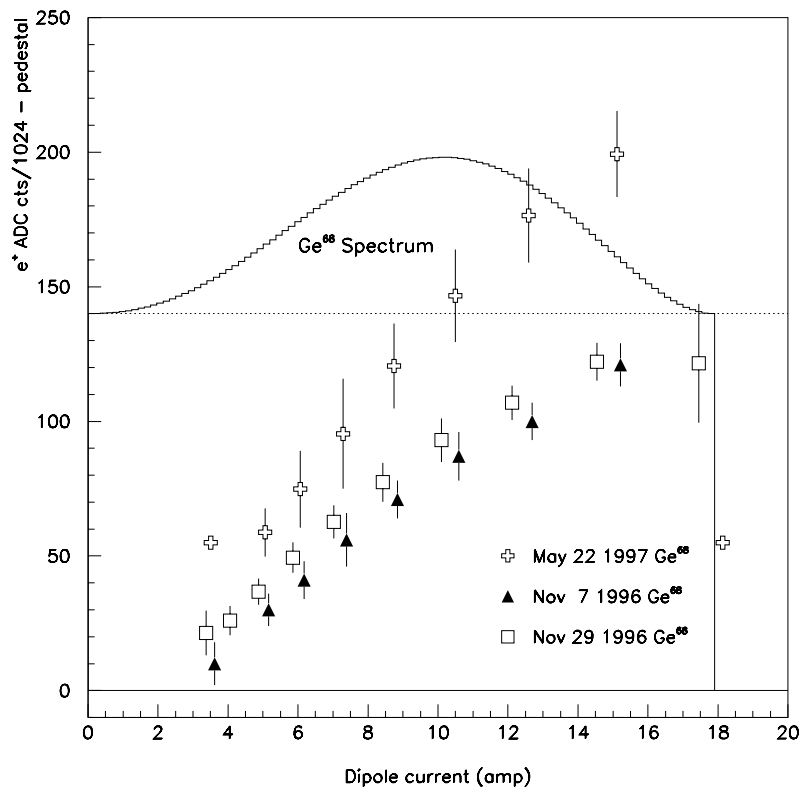

Figure 3.29: $\mathrm{e}^{+}$pulse height (ADC counts), pedestal subtracted with $\beta^{+}$spectrum of ${ }^{68} \mathrm{Ge}$ superimposed vs. dipole current.

Table 3.5: Fraction of events with NaI hits in the data.

\begin{tabular}{|c||c|}
\hline 3-way Candidates & \\
\hline Both ADC within $\pm 3 \sigma$ of $511 \mathrm{keV}$ peak & $30 \pm 7 \%$ \\
Both ADC strictly < peak & $38 \pm 7 \%$ \\
Only one is $>$ the peak & $17 \pm 5 \%$ \\
\hline $\mathrm{e}^{+}$calibration & \\
\hline Both ADC within $\pm 3 \sigma$ of $511 \mathrm{keV}$ peak & $23.8 \pm 2 \%$ \\
Both ADC strictly $<$ peak & $26.8 \pm 2 \%$ \\
Only one is $>$ the peak & $20.9 \pm 2 \%$ \\
\hline
\end{tabular}




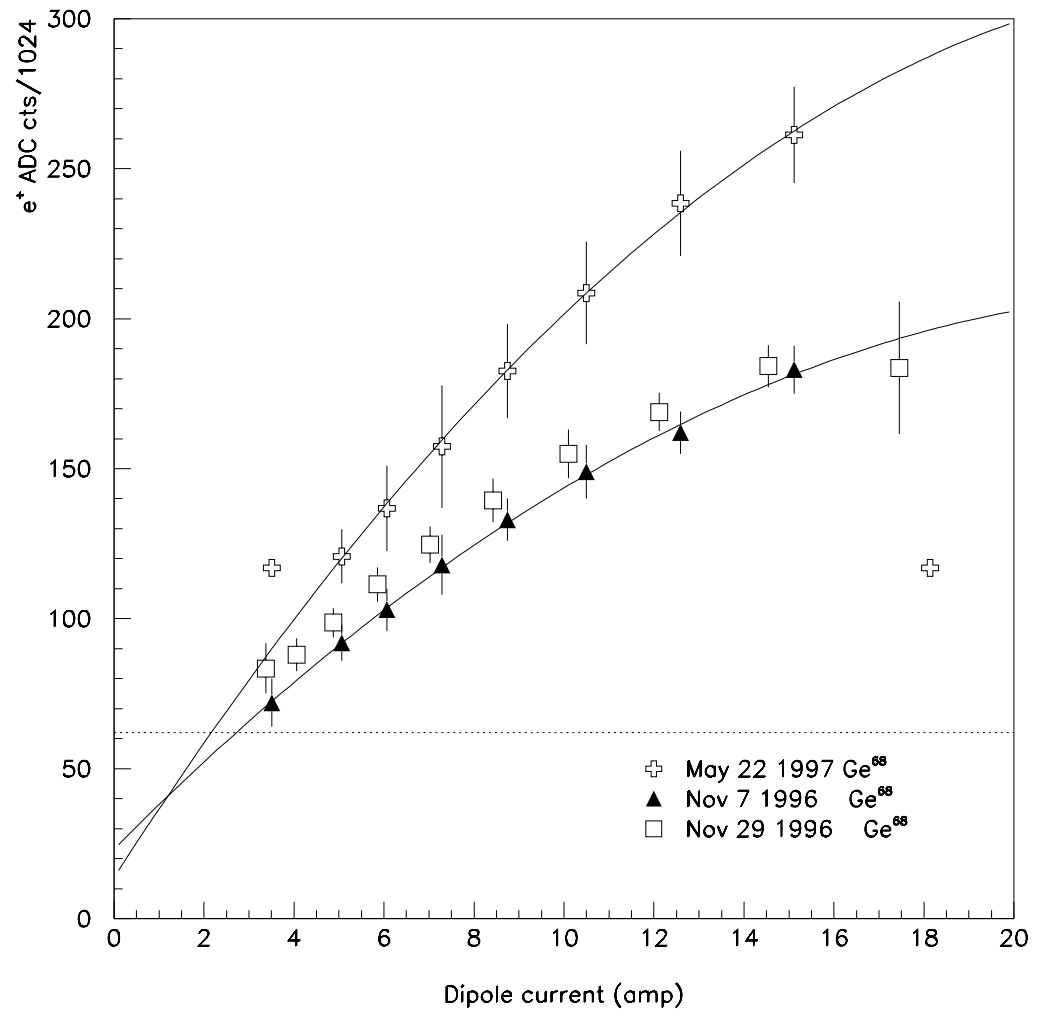

Figure 3.30: Compare gains of $e^{+}$plastic scintillators, dotted line is pedestal. 
Table 3.6: Fraction of events in the data with veto counter hits.

\begin{tabular}{|c||c|}
\hline $\mathrm{E}_{\gamma 1}<1 \mathrm{MeV}$ AND $\mathrm{E}_{\gamma 2}<1 \mathrm{MeV}$ \\
\hline V1 & $1.95 \%$ \\
V2 & $3.40 \%$ \\
V3 & $3.58 \%$ \\
V1 AND V2 & $0.67 \%$ \\
V1 AND V3 & $0.34 \%$ \\
V2 AND V3 & $0.71 \%$ \\
V1 AND V2 AND V3 & $0.16 \%$ \\
\hline
\end{tabular}

\subsection{Veto Counters and Shielding}

In front of the $\mathrm{NaI}$ can, plastic scintillator paddles form a veto counter identifying charged particles coming from the gas jet interaction region, (see figure 3.6). If there is only a hit in one of the paddles upstream of the NaI, chances are this was a background $\gamma$ or charged particle that started a shower, but never made it through the NaI. Two hits indicated a charged particle which kept going on through into the can. Most events with any veto hits had the 2 veto counters next to the $\mathrm{NaI}$ on and usually had large deposits in the halves. Hits were only used to veto offline. Even though the $\mathrm{NaI}$ can had a lead casing to catch gamma rays, they could create an electromagnetic shower in the lead and add extra energy to the NaI pulses.

Table 3.6 shows percentages of events with veto hits in association with $\mathrm{NaI}$ hits of a minimum energy (both ADC counts above pedestals). This would indicate that most of the low energy gamma rays are formed inside the hollow, lead cylinder. The calibration data from ${ }^{68} \mathrm{Ge}$, whose gamma ray energies are never above 250 counts (or $E_{\gamma}=1.1 \mathrm{MeV}$ ), show no veto hits at all. On the occasion that a charged particle leaves a hit in the upstream veto counters and gets through the lead, leaving near half an $\mathrm{MeV}$ in the $\mathrm{NaI}$ half and triggering the $\mathrm{e}^{+}$scintillator, the event could be vetoed. However, this was a rare occurrence. 


\subsection{Aperture Changes}

Various incremental changes in acceptance were made on the detectors to eliminate the question of whether antihydrogens were being lost or even if just the positron from antihydrogen were being lost. In January 1997, the original TF1 upstream counter was replaced by one with the same $2 " \times 2$ " square but only $1 / 16 "$ in thickness, decreasing the multiple scattering. The PWCs were recabled after run 19 yielding wider acceptance with reduced redundancy. During the March 1997 shutdown and most of April, the positron detector was modified to give it an increased acceptance. The 2" beam pipe upstream of the foil target (from the Y-pipe in A5B3) was replaced since the original had a slight vertical kink. The 1 " diameter foil was replaced with a 1.75" diameter foil. Unfortunately, during vacuum pumpdown, the nominal thickness foil was broken twice. Thus, for runs 51-115, corresponding to the latter half of the data-taking period, the $777 \mu \mathrm{g} / \mathrm{cm}^{2}$ thick (nearly double) foil, 1.75" in diameter, was used.

The sodium iodide cylinder was removed and sent back to the manufacturer to have the inner diameter increased from $1.3 "$ to $2.15 "$. The 1 "diameter positron scintillator was replaced by a 2" diameter plug, still $1 "$ long in the $e^{+}$direction of travel. This was coupled directly to the same 1" PMT with optical epoxy. Unfortunately, the modifications to the NaI took until June to be completed. The rest of the detector was tested with the sources in early May and the experiment started taking data, with an increase in rate seen immediately. Figures 3.31, and 3.32 show dipole current scans (holding the solenoid current fixed at the $\beta^{+}$spectrum peak) for both the high and low dispersion tunes. Notice they have not changed substantially from before the modifications were made. The May 1997 vertical scan of the source position, (see figure 3.33), shows what seems to be a loss of efficiency on the high angle side $\left(>300^{\circ}\right)$. The scan was done with the low dispersion tune and is to be compared 


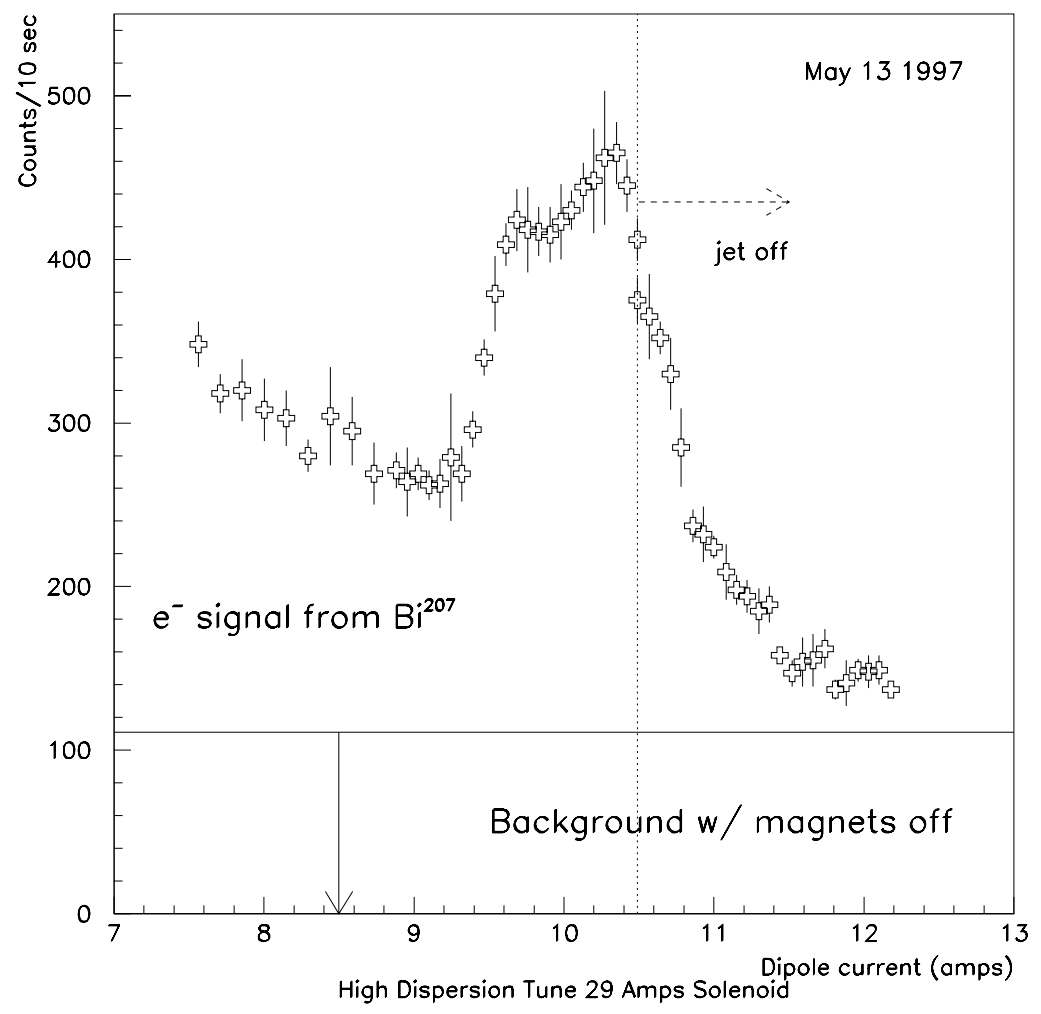

Figure 3.31: High dispersion dipole scan.

with figure 3.19. An identically shaped but reversed curve exists for the ${ }^{207} \mathrm{Bi}$ source from February. One might have expected that the scintillator would be imaged so that the new scintillator's larger size could be seen. The difference in apparent size did not rise in the ratio of $2: 1$ as one would have expected. This indicates the beam was not crossing a central diameter but is crossing a smaller length chord, (see figure $3.34)$. 


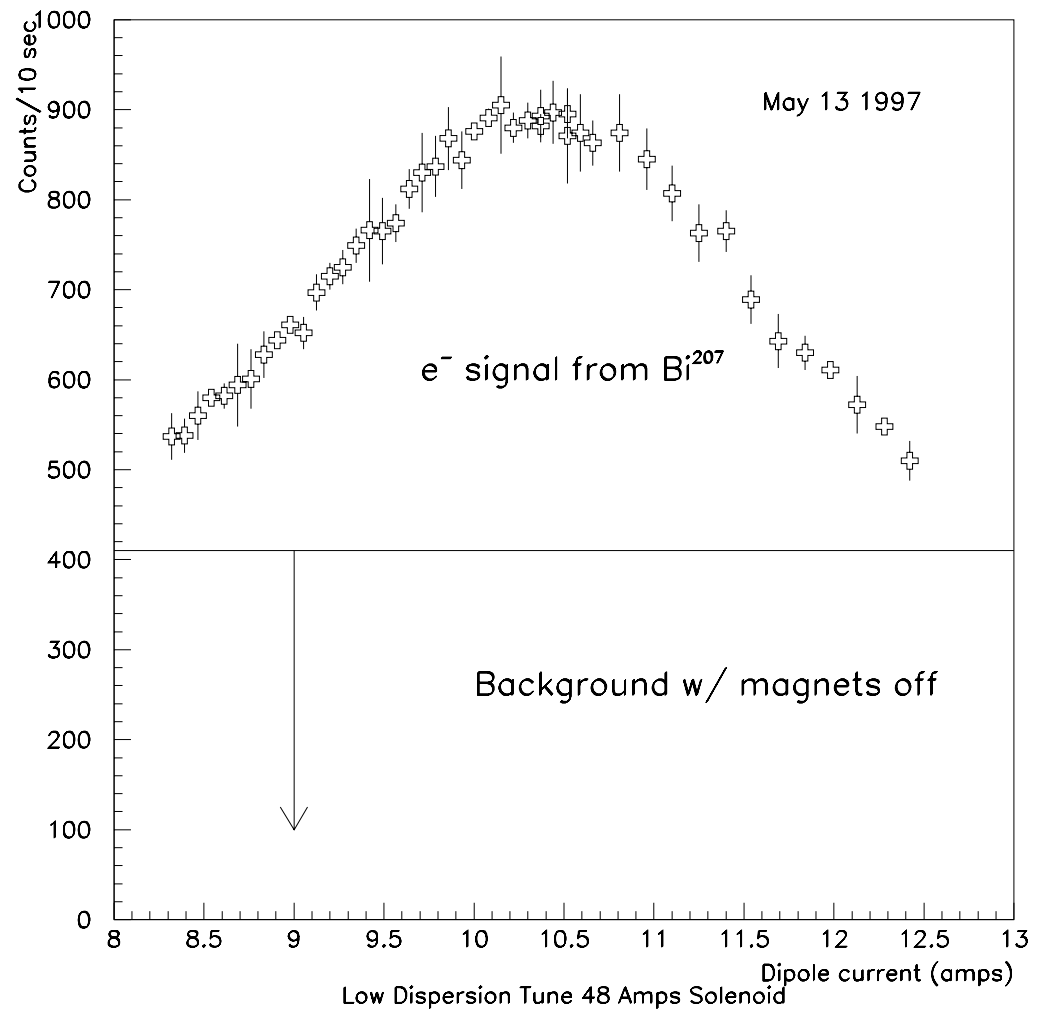

Figure 3.32: Low dispersion dipole scan. 


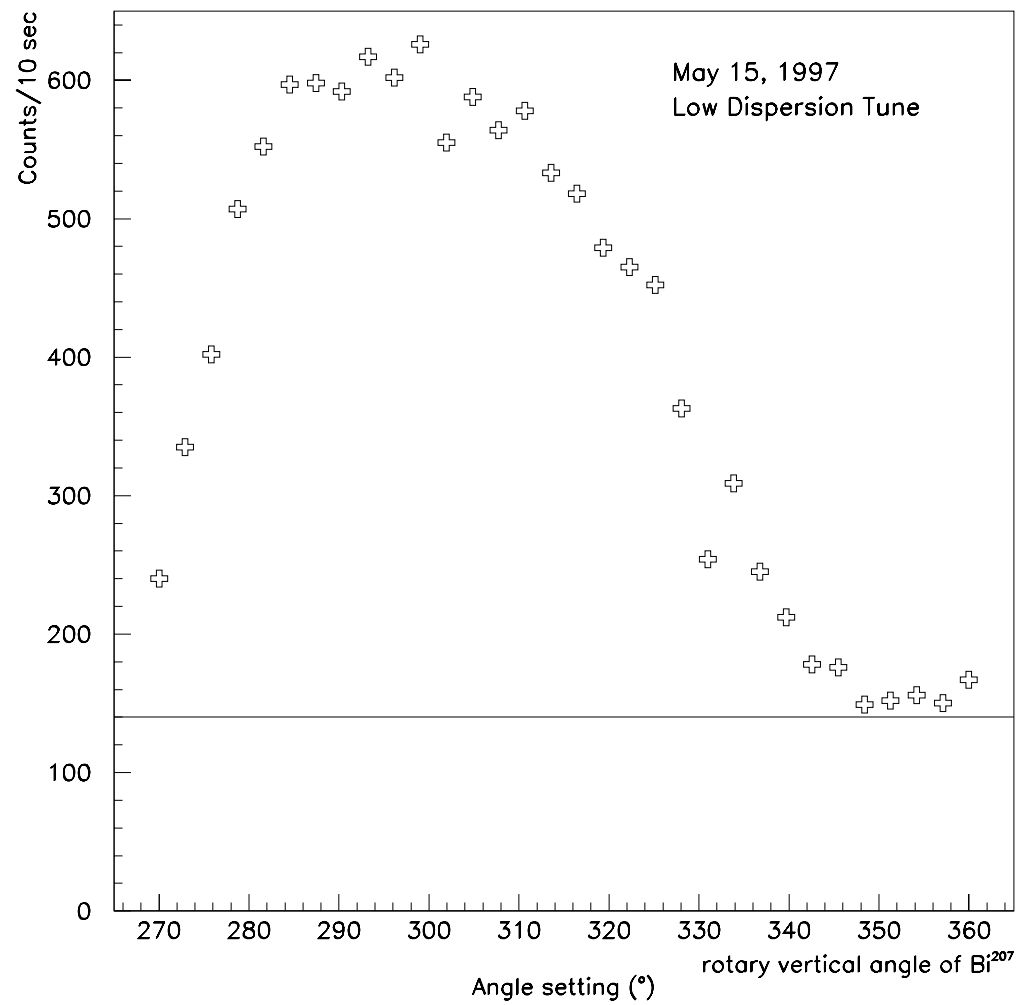

Figure 3.33: Vertical ${ }^{207} \mathrm{Bi}$ source position scan.

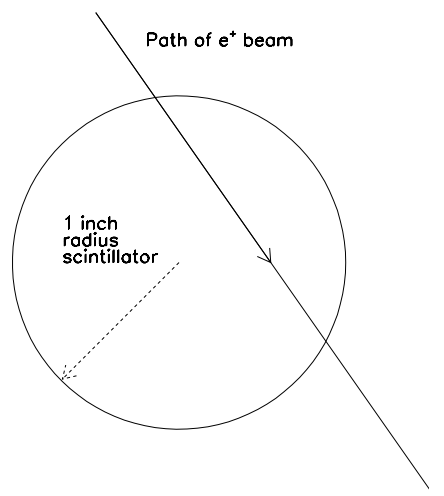

Figure 3.34: Positron beam crossing a chord of scintillator. 


\section{Chapter 4}

\section{The E862 Antiproton Spectrometer}

\subsection{Time of Flight}

Two fast plastic scintillator-fast phototube counters separated by 85 feet measured the time of flight of the antiproton. The time of flight clearly distinguished accidentals. The resolution was not enough to distinguish antiprotons from charged pions and kaons at the same beam momentum except at the lowest momenta. where the $\bar{n}$ could annihilate into a hadronic mode including pions. The pions would be significantly below beam momentum, closer to the $\bar{p}$ velocity. The resolution for on-momentum kaon rejection must be better than about 500 ps (FWHM) to get $3 \sigma$ rejection except for momenta $\mathrm{p}>8 \mathrm{GeV} / \mathrm{c}$, as seen in table 4.1. This table shows the differences in time of flight (nsec) between antiprotons and either $K^{-}$or $\pi^{-}$traversing time of flight counters separated by either 85 or 95 feet. The table considers that the kaons or pions are traveling at the same momentum. In other words no assumption has been made about the process from which they originate.

Fast Pilot-U plastic scintillator paddles, the upstream one $\left(2^{\prime \prime} \times 2^{\prime \prime} \times 1 / 8^{\prime \prime}\right)$, the downstream one $\left(4^{\prime \prime} \times 4^{\prime \prime} \times 1 / 2^{\prime \prime}\right)$ were separated by 85 feet. Coupled to two Amperex XP-2262 phototubes each, they provided timing resolution of:

$$
\sigma_{t}=\sqrt{\left(\sigma_{s c i n t}=350 p s\right)^{2}+\left(\sigma_{p m t}=350 p s\right)^{2}}=500 p s
$$


Table 4.1: Required resolution to distinguish $K^{-}$and $\pi^{-}$from $\bar{p}$ for equal momenta.

\begin{tabular}{|c||c|c|}
\hline \multicolumn{3}{|c|}{$\mathrm{K}^{-}$} \\
\hline $\mathrm{p}(\mathrm{GeV} / \mathrm{c})$ & $85^{\prime}$ & $95^{\prime}$ \\
\hline 3.7 & 2.707 & 3.026 \\
5.6 & 1.179 & 1.318 \\
5.9 & 1.075 & 1.202 \\
6.2 & 0.946 & 1.057 \\
8.9 & 0.466 & 0.521 \\
\hline
\end{tabular}

\begin{tabular}{|c||c|c|}
\hline \multicolumn{3}{|c|}{$\pi^{-}$} \\
\hline $\mathrm{p}(\mathrm{GeV} / \mathrm{c})$ & $85^{\prime}$ & $95^{\prime}$ \\
\hline 3.7 & 1.995 & 2.230 \\
5.6 & 0.868 & 0.970 \\
5.9 & 0.803 & 0.898 \\
6.2 & 0.700 & 0.782 \\
8.9 & 0.350 & 0.391 \\
\hline
\end{tabular}

The counters were tested with cosmic rays at Penn State to characterize the timing resolution. Figure 4.2 shows the setup used for the test. A pulse height correction was made to remove time walk from discrimination at a fixed threshold, (see figure 4.3). Two cables lengths of RG-58 were attached (30 feet, 80 feet) and FWHM of 500 ps was measured for the latter, (see figure 4.5), after pulse height correction. The cable lengths at Fermilab were $>300$ feet of RG- 8 , so the resolution was significantly worsened (1.6 ns FWHM) although not as much as would have been the case with RG-58. The final counters had these PMTs with voltage dividers, (see figure 4.1), modified for fast timing. The voltage applied to the phototubes was determined by measuring the efficiency (ratio of rate of the opposite PMT to coincidence rate of two) as a function of applied voltage, (see figure 4.6). There are two analog signals from each PMT base. The signal taken from the last dynode is sent upstairs as the ADC signal and inverted before being integrated. The anode signal has a larger pulse height than the dynodes and thus less error on the arrival time. The best precision on the timing pulse is obtained by discriminating after $10 \mathrm{~ns}$ of cable and sending the digital pulse upstairs. These signals arrive over long cables but the dispersion of the signals is known since the cable lengths are known. Unfortunately, the discriminator delay is not known precisely. In addition, in January, 1997, the TF1 signals were amplified $10 \mathrm{X}$ before being discriminated, adding extra indeterminacy 
Figure 4.2: Cosmic ray test time of flight counters 


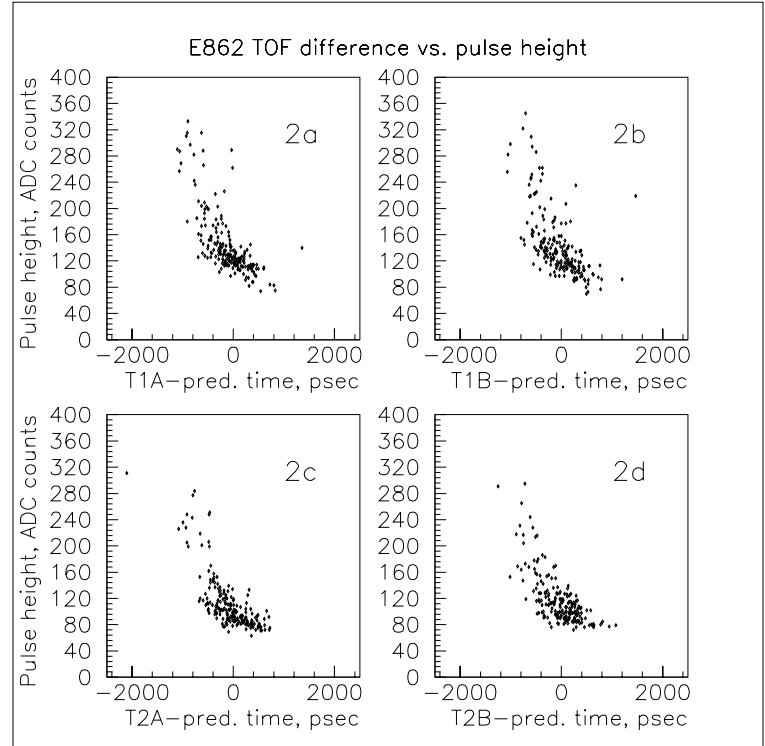

Figure 4.3: Time of flight difference showing the effect of time walk from pulse height differences.

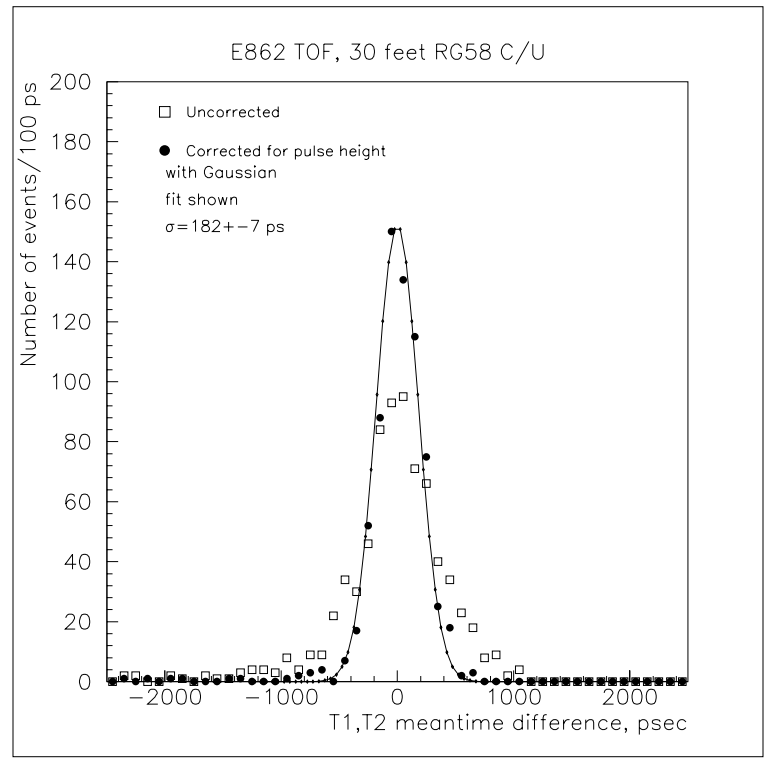

Figure 4.4: Time resolution for short, (30 ft) cables 


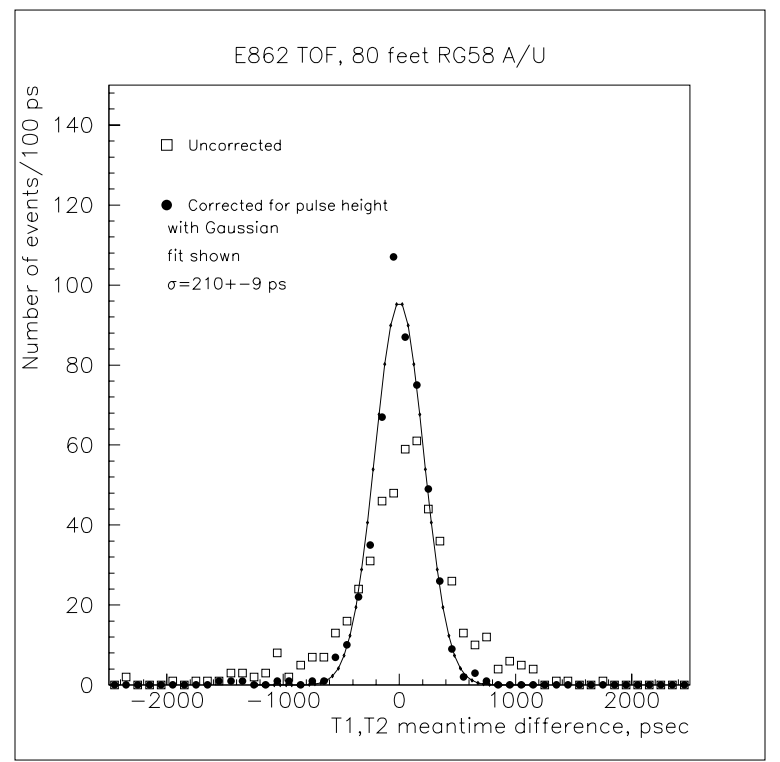

Figure 4.5: Time resolution for longer, (80 ft) cables
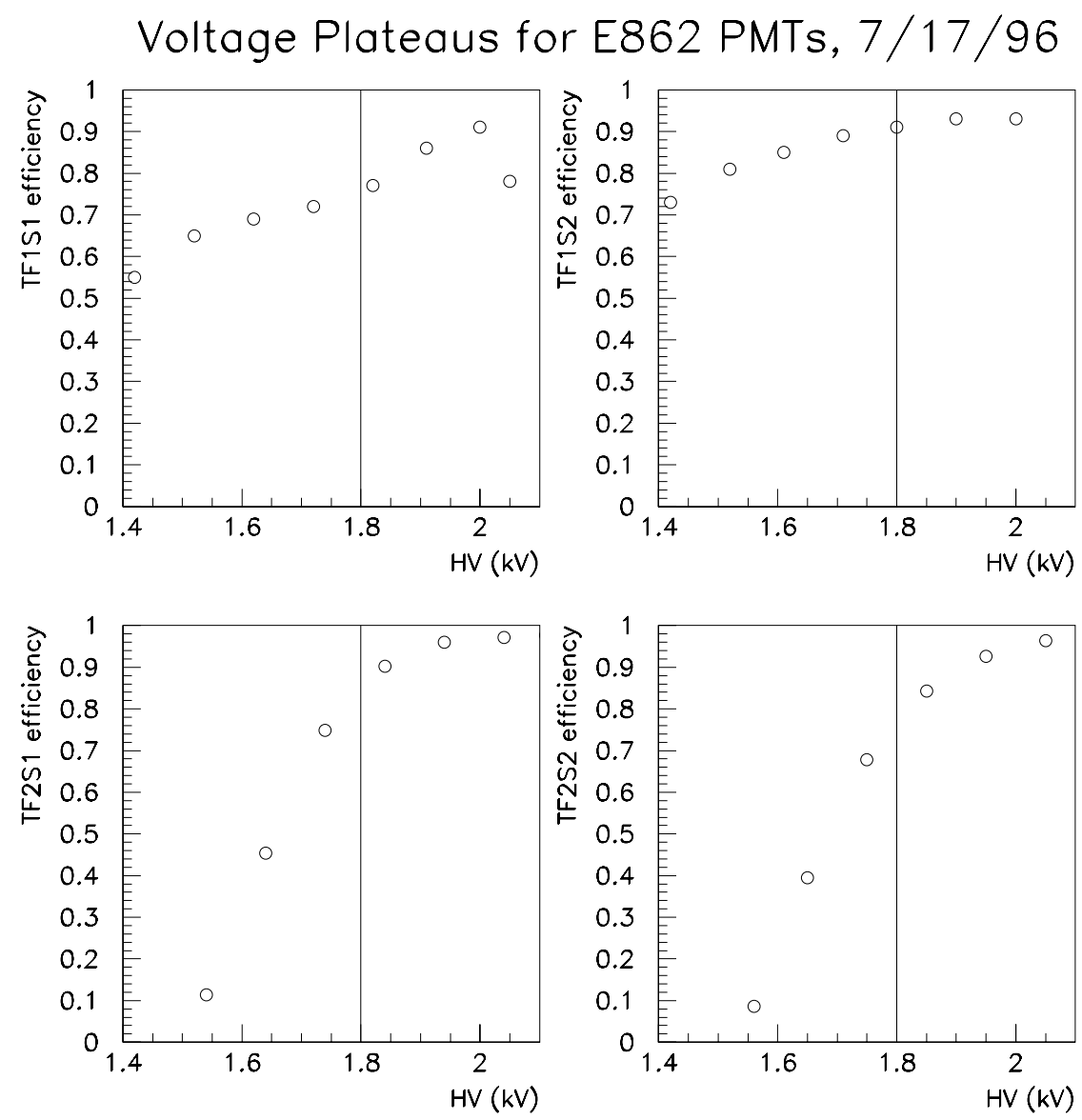

Figure 4.6: High voltage plateaus for the time of flight counters. 


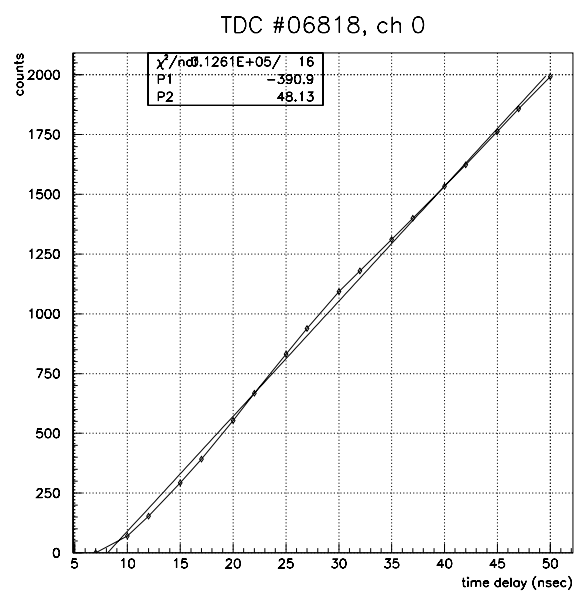

Figure 4.7: Nonlinearity of one of the fast-TDC channels. The average resolution of this channel is 21 psec per least count.

to the upstream timing distributions. This was done in order to increase the trigger rate by using all single electron noise $(5 \mathrm{mV})$ events in TF11 or TF12 in coincidence for part of the trigger.

The arrival times of the phototube signals from the plastic counters have uncertainties associated with the detector resolution as well as from the error on the conversion constants. A precision pulser was used to calibrate the TDC, and a sent time vs. measured time curve was fit to a line to determine TDC conversion constants, (see figure 4.7), for a typical channel's response. The signals from different times differ partly because of systematic errors arising from channel to channel differences in the TDCs $( \pm 1 \mathrm{ps} / \mathrm{LC})$ and each channel's nonlinearity (max: $\pm 5 \mathrm{ps} / \mathrm{LC}$, avg: $\pm 1.2 \mathrm{ps} / \mathrm{LC}$ ) which means different conversion constants should be used for different arrival times within the same channel. We assign one conversion constant of $21.2 \pm$ $1.5 \mathrm{ps} / \mathrm{LC}$ to all the TDC channels and arrival times. 


\section{$4.2 \quad$ Antiproton momentum measurement}

\subsubsection{Wire Chamber Description}

The momentum measurement of the antiproton after the first TOF counter uses two dipole bend magnets and three wire chambers. The dipoles have different lengths but roughly the same $\int B d L$, corresponding to a bend angle of about $5^{\circ}$, as shown in table 4.2.1. They are the only two spares for antiproton source ring magnets of their type available. Two dipoles instead of one increases the bend angle. The $\int B d L$ was uniform across the transverse dimension to less than $5 \times 10^{-4}$ over \pm 2.5 inches, (see figure 4.8), and as a function of applied bend bus current was linear to $10^{-5}$, (see figure 4.9). As discussed below, the main source of error in the momentum measurement was multiple scattering, not field nonlinearity.

Table 4.2: Momentum analysis magnet parameters. As described in section 4.3, the current settings had to be altered to center the beam.

\begin{tabular}{|c|c|c|c|c|}
\hline Magnet Type & $\begin{array}{c}\text { Bend Angle } \\
(\mathrm{mrad})\end{array}$ & $\begin{array}{c}\text { Arc Length } \\
\text { (inch) }\end{array}$ & $\begin{array}{c}\text { Radius of } \\
\text { Curvature (inch) }\end{array}$ & $\begin{array}{c}B_{\text {Max }} \\
(\mathrm{kG})\end{array}$ \\
\hline \multicolumn{6}{|c|}{ Ideal magnet usage } \\
\hline SDE-007 & 106.281 & 98.425 & 926.083 & 12.613 \\
SDD-070 & 95.077 & 65.370 & 687.549 & 16.988 \\
\hline \multicolumn{6}{|c|}{ E862 magnet usage } \\
\hline SDE-007 & 142 & & & \\
SDD-070 & 93 & & \\
\hline
\end{tabular}

Before, between, and behind the two dipoles are three multiwire proportional chambers which have rectangular-shaped active areas of $6^{\prime \prime} \times 4^{\prime \prime}$. They were used with a previous Fermilab experiment, E690 [88], as beam profile chambers and were characterized for efficiency, but at a higher pressure (15-30 psig) than our use at STP. They have $1 \mathrm{~mm}$ wire spacings, with wires arranged at angles of $\pm 7.1^{\circ}$ and $\pm 21.6^{\circ}$ 


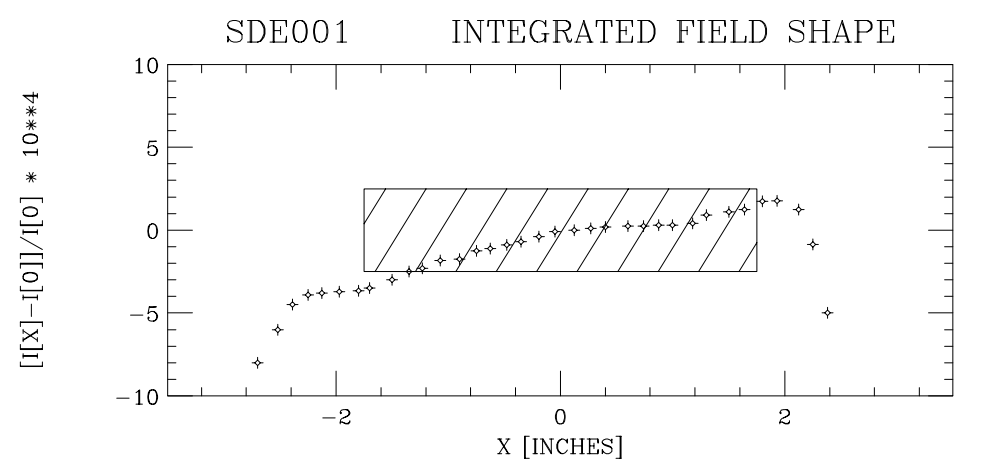

UP SCAN 19-DEC-1984 13:04:59.53 UP BASE 19-DEC-1984 13:16:56.

Figure 4.8: Characteristic nonlinearity of magnetic field across transverse dimension of an antiproton bend magnet [67].

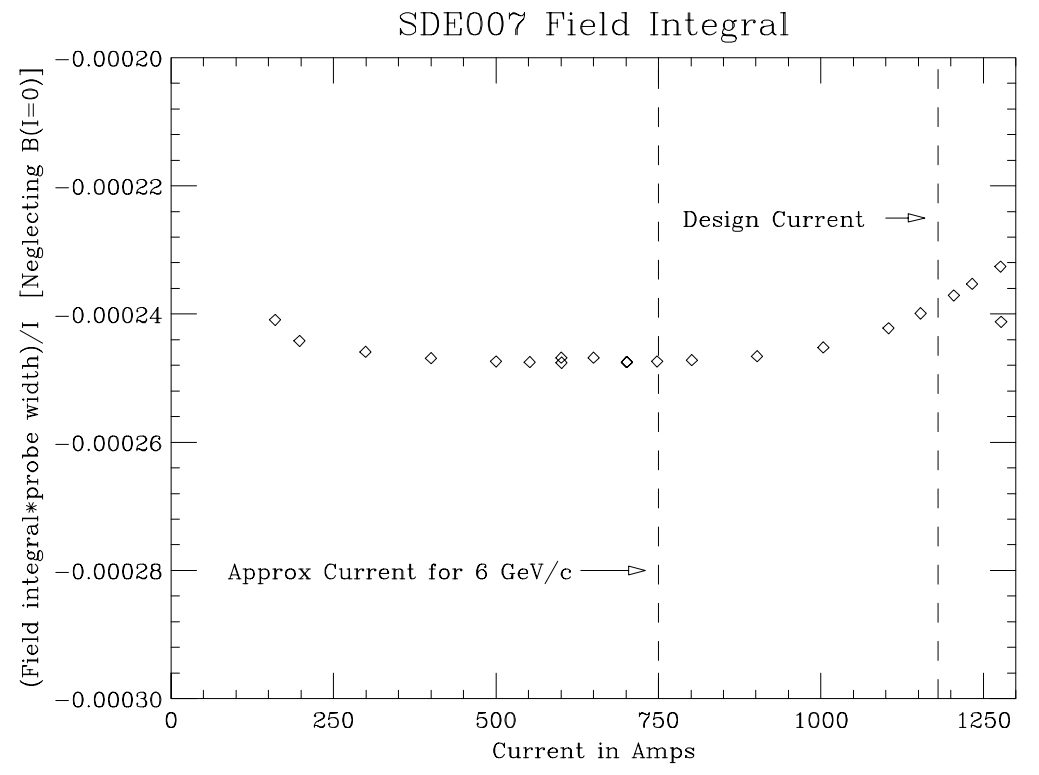

Figure 4.9: Nonlinearity of magnetic field as a function of applied current for an antiproton bend magnet. 
with respect to vertical. The pulse seen at the preamp is about $10 \mathrm{nsec}$ wide and has a jitter of about $30 \mathrm{nsec}$ due to the differences of arrival times of electrons to a wire.

The wire chambers had been assembled by E690 members by interleaving $0.001 "$ cathode foils in between signal wire planes and one foil each on either end of the sandwich along with one ground plane on either end, (see figure 4.11). G10 spacers were used between cathodes and signal planes. The planes were mounted on aluminum frames, one on each end. The other face of the frames were capped with Kapton/Kevlar windows designed to withstand high pressures.

The channels were read out with preamplifiers, (see figure 4.10), in groups of eight into discriminators located in crates in the tunnel near each chamber. The discriminators were set at $150 \mathrm{mV}$. The discriminator pulses were read out over ANSLI 32 channel flat delay cable which were between 150 and 260 feet long. The chambers are spaced 40 feet from each other. The signals from the delay cable arrive at signal conversion cards seated in standalone crates in the counting house. These converted the signals to new signals suitable for the LeCroy 4291 TDCs. The TDCs recorded one hit per trigger and were used in common stop mode. They were set to a resolution of $1 \mathrm{nsec}$ per least count for a total range of $512 \mathrm{nsec}$.

Since only one CAMAC crate of TDCs was available for use with the chambers, we could instrument only 704 channels. This put a constraint on which planes and sets of wires to use. Below is a table outlining the scheme best suited to measuring the momentum. 


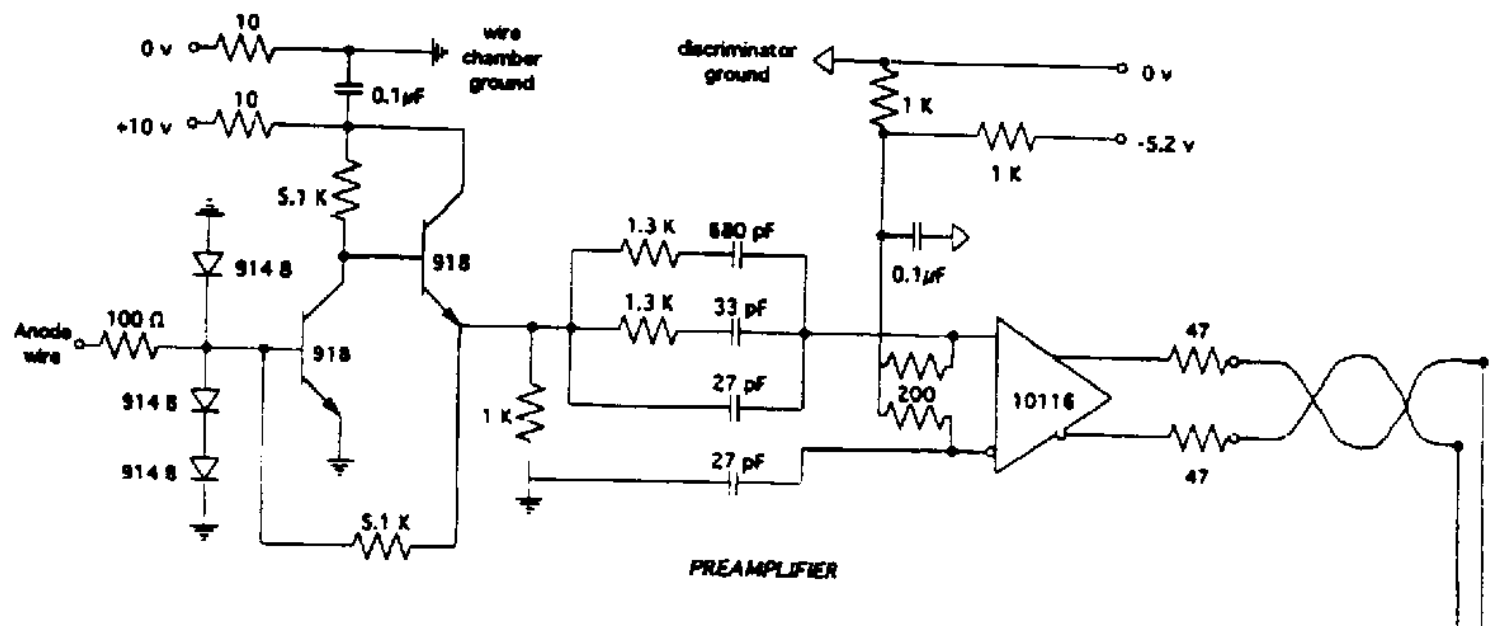

Figure 4.10: Preamplifier schematic for each PWC channel [88] 
Table 4.3: Wire chamber wires read out before run 10 .

\begin{tabular}{|c|c|c|c|}
\hline Chamber & Planes & $\begin{array}{c}\text { No. Wire/ } \\
\text { plane }\end{array}$ & $\begin{array}{c}\text { No. Wires /Plane } \\
\text { rel to beam }\left(\text { No. } \sigma_{\text {beam }}\right)\end{array}$ \\
\hline 1 & $1-4$ & 32 & $\pm 16\left( \pm \sigma_{\text {beam }}\right)$ \\
2 & $1-3$ & 64 & $\pm 32\left( \pm \sigma_{\text {beam }}\right)$ \\
3 & $1,3,4$ & 128 & $\pm 64\left( \pm 4.8 \sigma_{\text {beam }}\right)$ \\
\hline
\end{tabular}

Table 4.4: Wire chamber wires read out after run 9.

\begin{tabular}{|c|c|c|c|}
\hline Chamber & Planes & $\begin{array}{c}\text { No. Wire/ } \\
\text { plane }\end{array}$ & $\begin{array}{c}\text { No. Wires/Plane } \\
\text { rel to beam }\left(\text { No. } \sigma_{\text {beam }}\right)\end{array}$ \\
\hline 1 & 1,4 & 64 & $\pm 32\left( \pm 2 \sigma_{\text {beam }}\right)$ \\
1 & 2 & 32 & $\pm 16\left( \pm \sigma_{\text {beam }}\right)$ \\
2 & 2,3 & 96 & $\pm 32\left( \pm \sigma_{\text {beam }}\right)$ \\
3 & 1,4 & 128 & $\pm 64\left( \pm 4.8 \sigma_{\text {beam }}\right)$ \\
& 3 & 96 & $\pm 48\left( \pm 3.6 \sigma_{\text {beam }}\right)$ \\
\hline
\end{tabular}




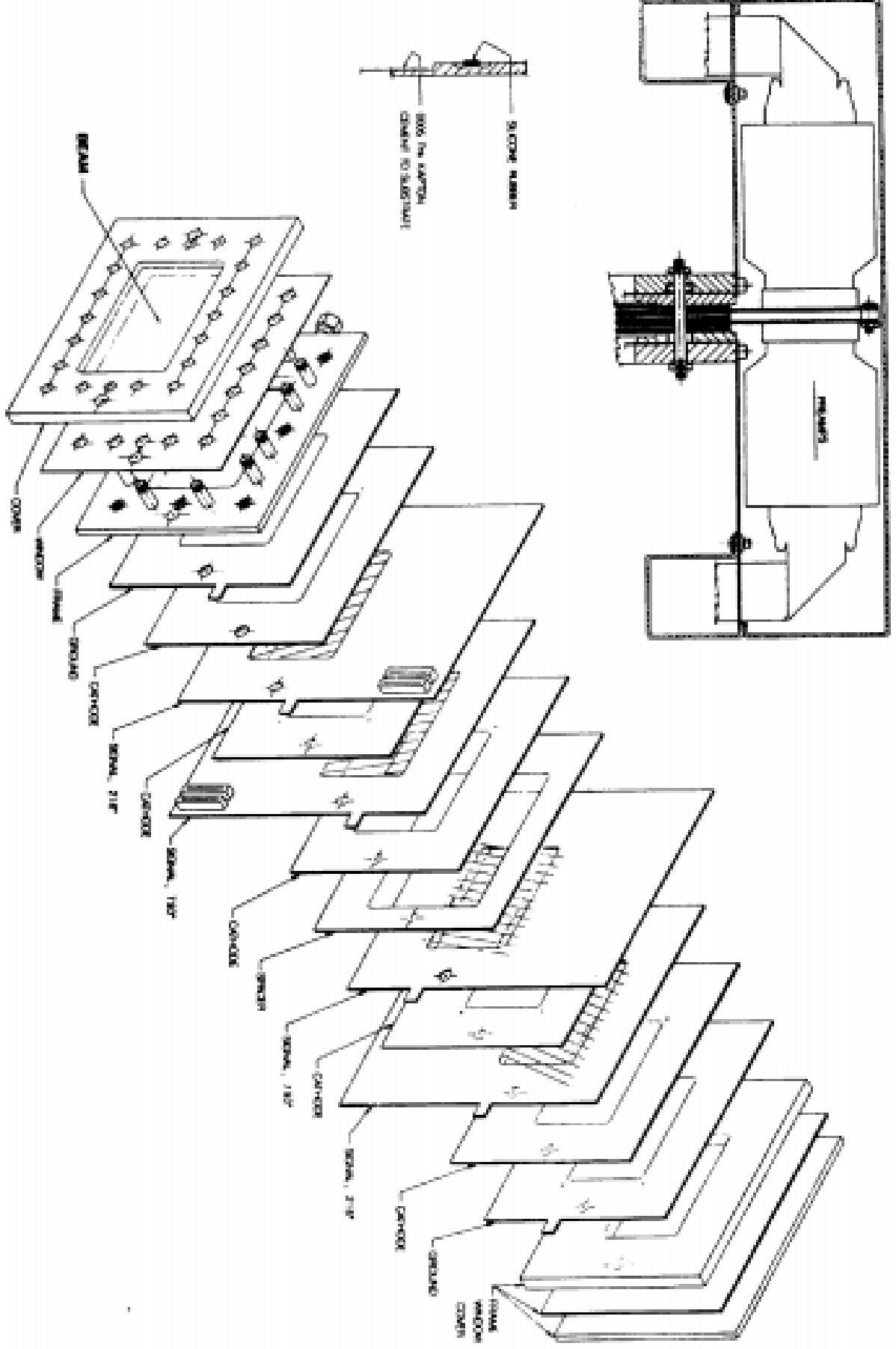

Figure 4.11: Breakdown showing the various planes of the wire chambers [88] 
Before commissioning the wire chambers, small spots of residue from beaminduced radiation damage were cleaned from the planes. New cathode foils were glued on the frames and the wires were cleaned with solvent around the area where residue was built up. Finally, a gain measurement was made to ensure equal gains for all wires in a plane.

\subsubsection{Momentum Resolution}

The goal of the momentum measurement was to be able to resolve the onmomentum antiprotons from antihydrogen from other tracks to a few times the beam momentum spread of $\frac{\delta p}{p}=2 \leftrightarrow 5 \times 10^{-4}$. Material along the path gives the $\bar{p}$ an angular spread from multiple Coulomb scattering. Because the track angle changes in the wire chambers due to multiple scattering, the momentum resolution is degraded. The required momentum resolution sets a limit on the thickness of the TOF upstream scintillator paddle, since the paddle was downstream of the first wire chamber and the thickness of the chamber foils and target foils are fixed. It is a small effect, though, and the primary reason for a thin counter is to have a small spot size on the wire chambers downstream. Its thickness was 0.25 " until run 21 when it was replaced with a 0.125 " thick paddle. There is also a 0.002 " titanium exit window at the end of the high vacuum region. Table 4.2.2 lists the contributions from multiple scattering from the various materials. The end result is a best momentum resolution of $10^{-3}$ for a $3.672 \mathrm{MeV} / \mathrm{c}$ antiproton: 
Table 4.5: Multiple scattering contributions at $3.7 \mathrm{GeV} / \mathrm{c}$ and $6 \mathrm{GeV} / \mathrm{c}$.

\begin{tabular}{|c|c|c|c|c|c|c|}
\hline \multirow[t]{2}{*}{$\mathrm{z}(\mathrm{m})$} & \multirow[t]{2}{*}{ Location } & \multirow[t]{2}{*}{$\overline{X_{0}}$} & $\begin{array}{c}\sigma_{y} \\
(\mathrm{~mm})\end{array}$ & $\begin{array}{c}\sigma_{\theta_{y}} \\
(\mathrm{mrad})\end{array}$ & $\begin{array}{c}\sigma_{y} \\
(\mathrm{~mm})\end{array}$ & $\begin{array}{c}\sigma_{\theta_{y}} \\
(\mathrm{mrad})\end{array}$ \\
\hline & & & \multicolumn{2}{|c|}{$3.7 \mathrm{GeV} / \mathrm{c}$} & \multicolumn{2}{|c|}{$6 \mathrm{GeV} / \mathrm{c}$} \\
\hline 0 & Jet (A50) & & 1.40 & 0.240 & 1.40 & 0.240 \\
\hline 15 & A5B3 exit & & 3.86 & 0.240 & 3.86 & 0.240 \\
\hline 18 & & & 4.54 & 0.240 & 4.54 & 0.240 \\
\hline 18 & $400 \mu \mathrm{g} / \mathrm{cm}^{2}$ & $9.37 \cdot 10^{-6}$ & 4.54 & 0.240 & 4.54 & 0.240 \\
\hline 19 & & & 4.77 & 0.240 & 4.77 & 0.240 \\
\hline 19 & Ti window, $0.002 "$ & 0.000937 & & & & \\
\hline & $0.35 "$ air & 0.000329 & & & & \\
\hline & PWC (4 planes) & 0.00153 & & & & \\
\hline & $1 / 4 "$ scintillator & 0.00748 & & & & \\
\hline & 4.2 mil Mylar & 0.000376 & & & & \\
\hline & TOTAL & 0.01084 & 4.77 & 0.402 & 4.77 & 0.310 \\
\hline 37 & & & 5.75 & 0.402 & 5.38 & 0.310 \\
\hline 37 & PWC & 0.00153 & 5.75 & 0.417 & 5.38 & 0.317 \\
\hline 55 & & & 6.65 & 0.417 & 5.94 & 0.317 \\
\hline 55 & PWC & 0.00153 & 6.65 & 0.432 & 5.94 & 0.324 \\
\hline
\end{tabular}

\subsection{Antiproton Beamline Alignment}

Before the antihydrogen running began, two calibration periods, consisting of 12 hours of dedicated antiproton beam down the E862 beamline, were used to align the wire chambers. The chambers had been aligned on the beamline optically to a precision of $1 \mathrm{~mm}$, but the calibration periods provided a verification using real beam. One $2 \mu \mathrm{sec}$ shot of protons was projected on the antiproton target. That shot was immediately transferred to the debuncher and then to the accumulator, where the dipole A5B3 was shunted off so that antiprotons would travel straight into the E862 apparatus. The target wheel was moved to a blank for fear of radiation damage to the foils. The small dipole was left off since the deflection at the calibration momentum of $8.85 \mathrm{GeV} / \mathrm{c}$ would be different than at the run momenta.

The large antiproton bend dipoles downstream were set for $8.85 \mathrm{GeV} / \mathrm{c}$. The wire chambers' high voltage was set at $-1350 \mathrm{~V}$. Because the TDCs are used nominally 
with a 512 nsec gate, less than a quarter of the shot's antiprotons were able to be recorded.

The first calibration period proved the readout of the PWC TDCs in the DAQ worked properly but the distributions in transverse dimension and in time were flat in PWC \#1 and PWC \#2 and no sign of a beam spot was seen. In addition, PWC \#3 had a much lower rate. After the period was over, it was discovered that, a vacuum isolation valve had been closed upstream. The beam was hitting the valve and spraying secondaries down the E862 beamline. Secondly, one of the antiproton dipoles in the E862 beamline was steering the beam off by $1.3^{\circ}$ because it was designed for somewhat less current. To compensate, the beampipe downstream of the bend magnets was moved to the outside by $1.3^{\circ}(36.9 \mathrm{mrad})$.

The second calibration period was more successful. The same procedure of datataking was used. The resulting distributions across wires in a plane showed a beam spot within the apertures of each chamber. The distribution of all hit times shows 3 peaks, one for each chamber, since the delay cables were such that each chamber's hit times were separated from the others' by about 100 ns for a beam momentum particle. The delay for the common stop signal on the PWC TDCs was set so that the last quarter of the bunch was seen. Even so, the number of hits was large enough that forming space points and tracks was difficult. For subsequent tests, the beam was scraped down in intensity (and in size). Events were recorded at about $3 \mathrm{~Hz}$, although each event had a couple of hundred tracks in the $500 \mathrm{~ns}$ window. About 30 events were accumulated in each run.

Once this had been established, various local deflections within the ring (or orbit distortions) upstream of A50 were introduced to characterize where the beam spot was located within the chambers and when the beam hit the edges of the chambers or upstream apertures. Orbit distortions in angle and position for both the horizontal 
Table 4.6: Left and right edges of the beam (inch)

\begin{tabular}{|c||c|c|c|c||c|c|}
\hline Perturbation & X-left & X-right & Y-left & Y-right & $|\Delta X|$ & $|\Delta Y|$ \\
\hline Standard Stacking & -.223 & -.498 & -.126 & .104 & .275 & .230 \\
Settings & & & & & & \\
Horizontal Angle & -.236 & -.291 & .199 & .072 & .055 & .127 \\
Horizontal Angle & -.159 & -.554 & -.153 & .001 & .395 & .154 \\
Horizontal Angle & -.237 & -.503 & .094 & -.167 & .266 & .261 \\
Horizontal Position & -.229 & -.432 & .111 & -.001 & .203 & .112 \\
Horizontal Position & -.239 & -.315 & .089 & .078 & .076 & .011 \\
Vertical Angle & -.231 & -.356 & .136 & -.336 & .125 & .472 \\
Vertical Angle & -.220 & -.464 & .007 & .063 & .244 & .056 \\
Vertical Position & -.175 & -.518 & -.166 & .095 & .343 & .261 \\
Vertical Position & -.203 & -.430 & .058 & -.205 & .227 & .263 \\
Standard Settings, & -.194 & -.454 & -.078 & -.101 & .260 & .179 \\
e dipole ON & & & & & & \\
E835 angle & -.171 & -.375 & -.180 & .099 & .204 & .279 \\
E835 angle removed & -.205 & -.507 & .062 & -.061 & .302 & .123 \\
septum 1 voltage & & & & & & \\
septum 2 voltage & -.202 & -.419 & -.021 & .134 & .217 & .155 \\
kicker voltage & -.164 & -.426 & .266 & .330 & .262 & .064 \\
\hline
\end{tabular}

and vertical dimensions were tried. Changes in septum voltages and kicker currents were also tried. Shown in figure 4.12, are characteristic wire number profiles (across one plane) and a time profile, one peak for each chamber. Shown in table 4.3, are the edges of the beam ascertained from the wire numbers of each plane which defined a FWHM of the distribution across wire number in each plane. Each orbit distortion or other perturbation is listed with the corresponding effect on the beam edges.

Finally, the last few tests were used to change the gains of the PWCs. Five runs were taken at differing chamber voltages to get a rough look at the chamber efficiencies as a function of voltage. A voltage of $-1350 \mathrm{~V}$ for all three chambers was initially chosen as the operating point, although runs $10-13$ used $-1375 \mathrm{~V}$ and starting with run $14,-1400 \mathrm{~V}$ was used throughout. 


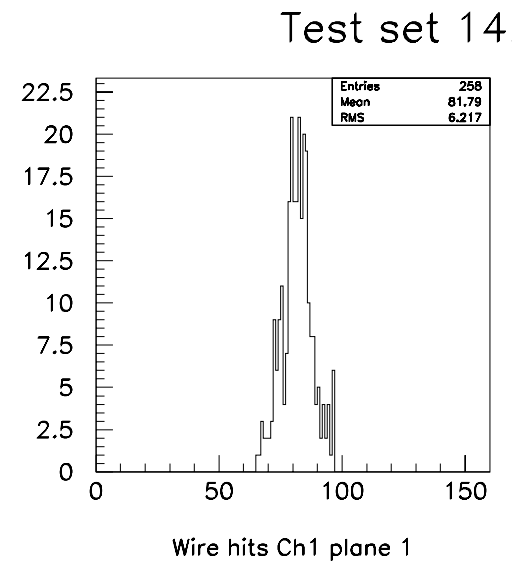

19, 1996
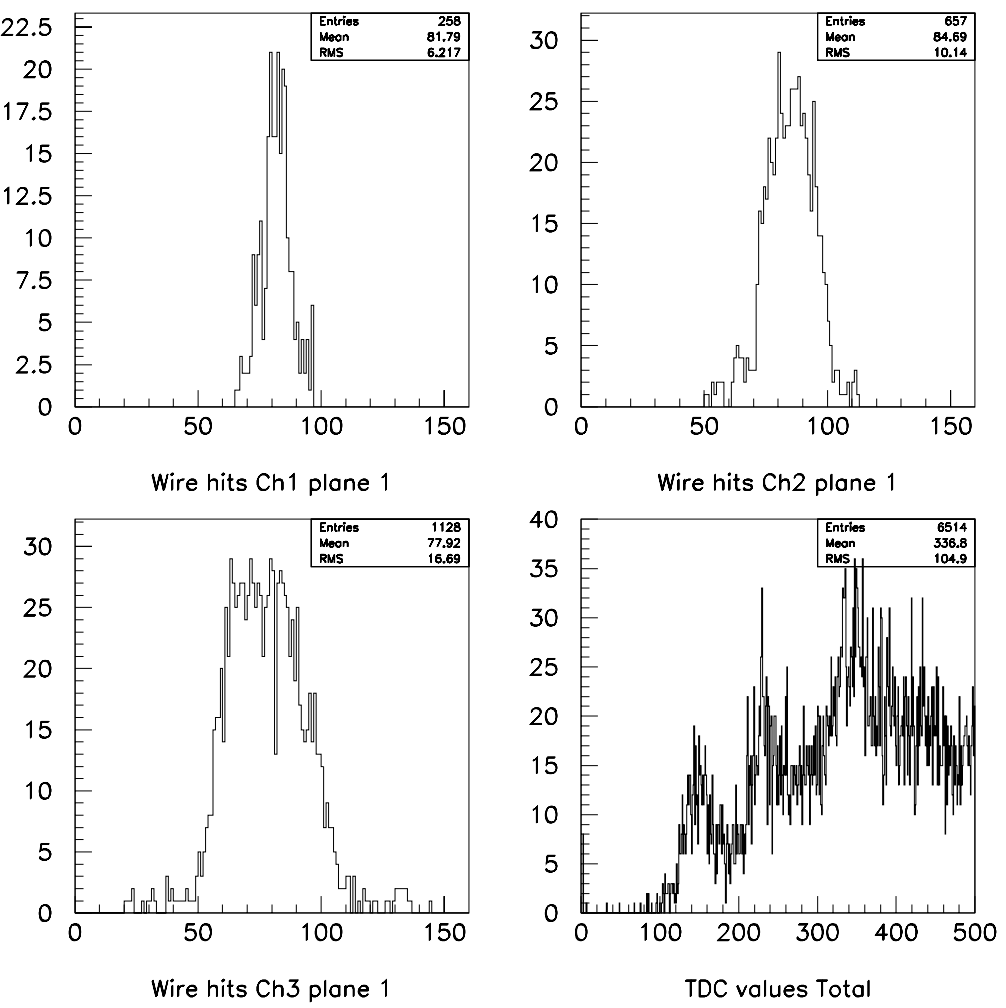

Figure 4.12: Typical transverse transverse profiles by wire number for each PWC and time distribution of hits showing 3 peaks arising from different cable delays for the 3 chambers. 


\section{Chapter 5}

\section{Trigger and Data Acquisition}

\subsection{Experiment Trigger}

The basic antihydrogen requirement is to see a hit in the positron detector's counter AND to see a hit in both of the time-of-flight counters. This three-fold requirement was relaxed to less restrictive triggers consisting of the two-fold coincidences of scintillator paddles. This allowed a more thorough characterization and rejection of background in offline analysis. The coincidence of the two phototubes' signals from each time of flight counter, named TF1(upstream) and TF2(downstream), reduced random noise since the raw rate of each phototube was on the order of 1 kHz. The TF2 coincidence (TF21 AND TF22) was made after the signals left the PMT bases, travelled over $10 \mathrm{~ns}$ of cable and were discriminated. The individual, discriminated signals went to TDCs, but the coincidence was sent over Heliax cable $(\mathrm{v}=0.88 \mathrm{c}$ ), high speed cable (same signal dispersion as RG-8), upstairs to arrive closer in time with the upstream signals and eliminate delay cable. The signal from TF1 was used in all triggers except $\mathrm{H} 4$ and was delayed to come second in time so it would act as a time strobe. The signals in the coincidence triggers were set to overlap by 75 nsec. Three antihydrogen triggers were built upon this:

- H1: $T F 1 \otimes e^{+}$ 
- H2: $T F 1 \otimes T F 2$ - This was the most restrictive needing all 4 TF PMTS. No hit in the $\mathrm{e}^{+}$arm is required so background tracks could be seen with this trigger.

- H3: $T F 1$ (prescaled) (usually by a factor of 100). The prescale factor was determined so that the live time would be maximized for real antihydrogen events.

- H4: $T F 2 \otimes e^{+}-$Added after run 20 to see whether any antihydrogen events were missing TF1 completely. Since it was not strobed to TF1, its absolute timing was only close to the other triggers to within about $1 \mathrm{nsec}$.

Then the hits from all pmts except those attached to the $\mathrm{NaI}$ were read out as a random PMT trigger (A1) in order to assess pedestals, etc.

- A1: $E 1 \oplus T F 1 \oplus T F 2 \oplus T 3 \oplus T 4 \oplus V 1 \ldots$

Finally, to test the positron arm, an $\mathrm{e}^{+}$counter only trigger (A2) was used to accumulate statistics when using the ${ }^{68} \mathrm{Ge}$ source, especially since this could be done without switching the power supply lugs.

- A2: $e^{+}$only

The rates from these triggers (averaged over runs) are shown in table 5.1:

The rates of the counters themselves scale with luminosity. The luminosity in the following, (see figure 5.2), is calculated from the beam current and jet density at the time. The $\mathrm{NaI}$ is a coincidence between the $\mathrm{NaI}$ halves where both are discrimiated at the $250 \mathrm{keV}$ level. TF1 and TF2 are coincidences between the two phototubes attached to each time of flight counter. 


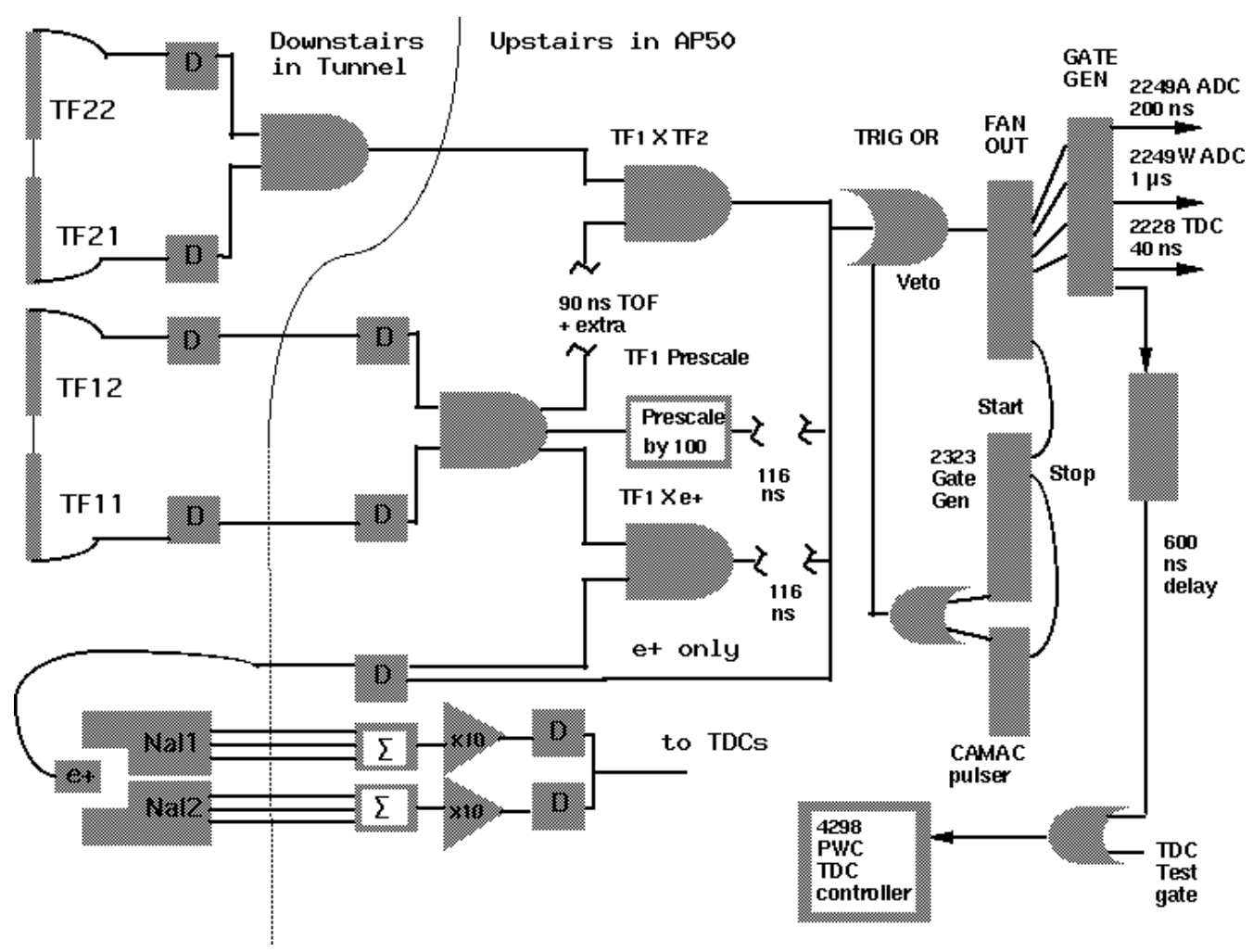

Figure 5.1: E862 trigger diagram.

Table 5.1: Overall E862 trigger rates for normal running conditions (beam).

\begin{tabular}{|c|c|}
\hline Trigger & Rate $(\mathrm{Hz})$ \\
\hline $\mathrm{H} 1$ & $6 \times 10^{-3}<R<2 \times 10^{-2}$ \\
$\mathrm{H} 2$ & $5 \times 10^{-4}<R<3 \times 10^{-3}$ \\
$\mathrm{H} 3$ & $1 \times 10^{-2}<R<7 \times 10^{-2}$ \\
$\mathrm{H} 4$ & $10^{-5}<R<10^{-4}$ \\
$\mathrm{~A} 2$ & $4<R<20$ \\
\hline
\end{tabular}


Variation of rates with luminosity (from jet, beam)
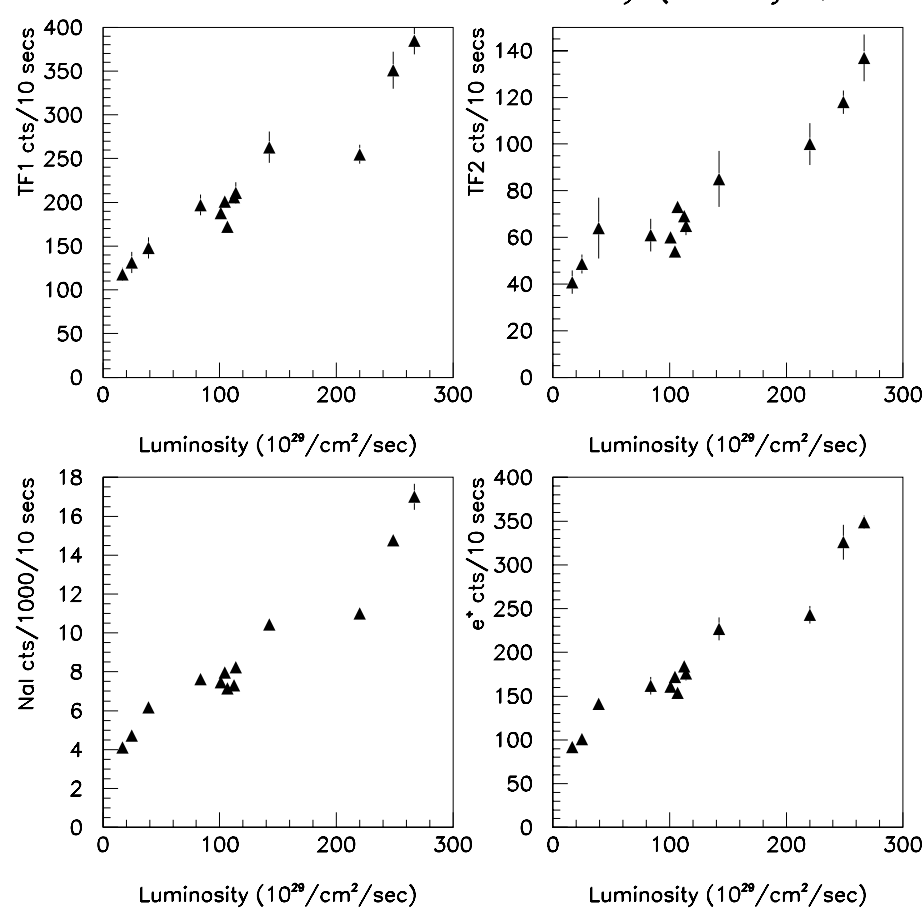

Figure 5.2: Average rates from the counters as a function of instantaneous luminosity.

The trigger was built completely from commercial NIM standard modules. CAMAC scalers were used to read out, as well as Fermilab-built visual scalers used for rates.

\subsection{Data acquisition}

The channels consist of counters reading out to ADCs and TDCs and chamber TDC hits. The expected rate of events for random coincidences contributing to the $T F 1 \otimes T F 2$ trigger rate is $10^{-5} \mathrm{~Hz}$. Using the other triggers allowed distributions of measurements for background channels to be acquired. Data was taken at a rate of a few Hz. An Apple Macintosh computer read out events through a Jorway CAMAC interface. At this low rate, there was an average $97.0 \%$ live time. The live time is the ratio of total triggers to triggers recorded by the data acquisition. Most of the 
dead time came from bursts of beam (few hundred/sec) during periods of significant beam loss when the data acquisition could only record a few events per second.

Every ten events, scaler channels recording rates of individual counters as well as trigger rates were written along with high voltages into the datastream. Every 15 minutes, a unix workstation polled the Macintosh for new data files and transferred the files to the workstation for writing to tape and analysis.

A preliminary analysis was performed on the data consisting of track forming and cutting on expected energy and time windows. Events passing loose cuts were dumped to file and made available via the experiment web site. Antihydrogen candidates, as selected by triple coincidence, triggered a pager, as did alarms on high voltage and data acquisition errors. In all, 129178 events resulting from jet-beam interactions have been acquired and written to disk.

\subsection{Target and Magnet Control}

The control subsystems, run through the accelerator's ACNET interface and network, made easy access for changing the position of the stripper wheel and controlling the current through the magnets, (see section 2.1.3). The ACNET interface page could be brought up on a workstation and issued commands to read or change parameters.

The dipole (60A at $10 \mathrm{~V} \max )$ and solenoid (160A at $30 \mathrm{~V} \max$ ) power supplies could be operated in both voltage and current regulated modes. The power supplies were controlled from (two) 0-10V analog levels from a 16 bit DAC (digital-analog converter) which sat in a CAMAC crate near the supplies. While antihydrogen data was being taken, the magnets needed to track the beam energy and, thus, the rest 


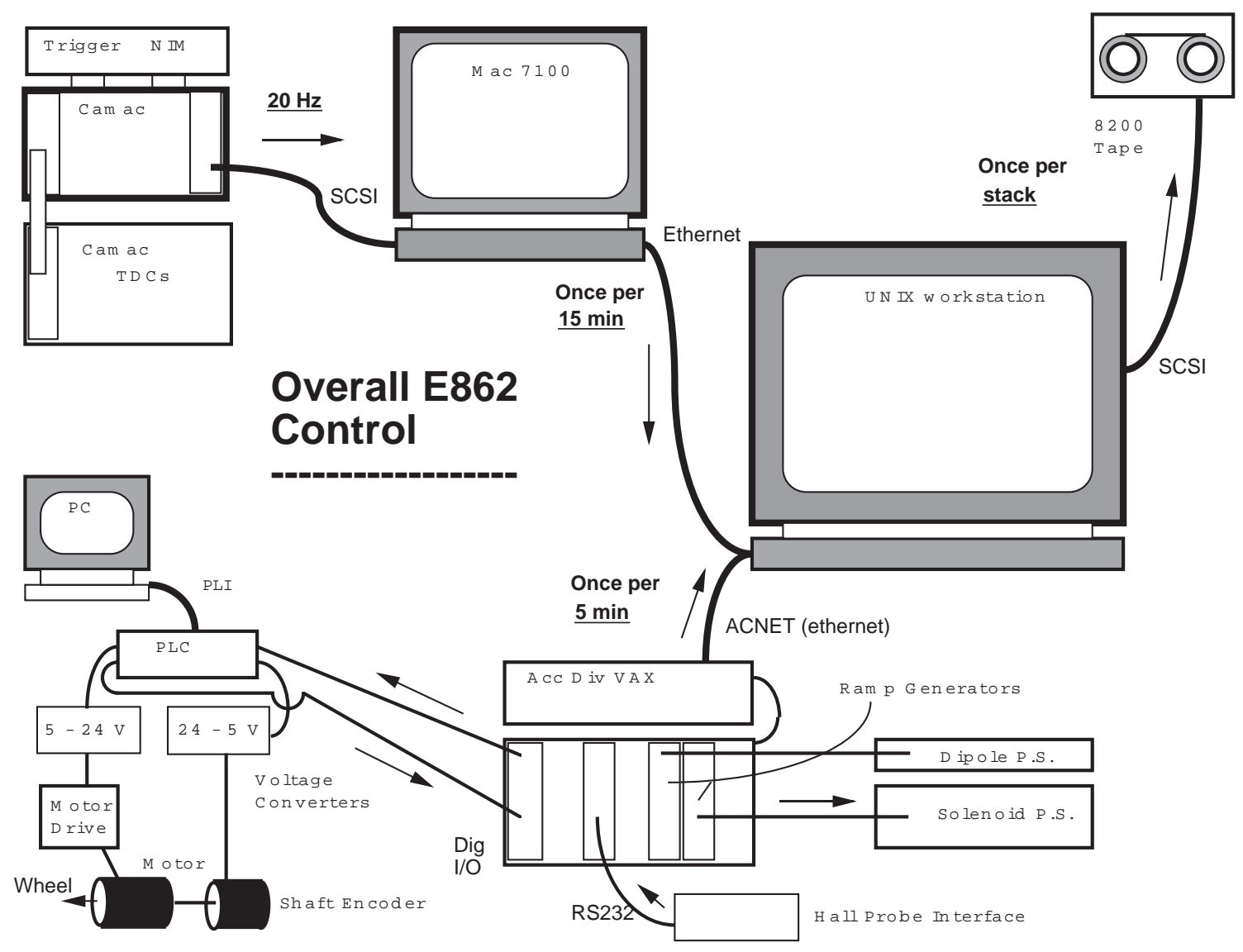

Figure 5.3: Data collection path and control of the magnets and target wheel. 
of the ring's magnet currents which were available from a table setup on ACNET. When a radioactive source was put in place, the current could be reset to that needed to bend the positrons or electrons from the sources. Every time the electron source was used, the polarity of the leads had to be reversed on both supplies by hand. As described in section 3.5.3, the dipole had significant hysteresis so it had to be ramped back and forth a few times before settling down to a steady state I vs. B profile. The currents were read out by a scanning, multiplexed ADC.

The target wheel was attached to one end of the shaft of a stepper motor. On the back end of the motor, a shaft encoder recorded rotation angle changes at the same resolution of 400 counts per revolution. This affects the resolution of the foil vertical position. A feedback loop was implemented by using a micro programmable logic controller to compare the number of steps seen to that given to the motor. When a new position was requested, the wheel would be advanced to a zero point at which the encoder sent out a pulse and then would be advanced the needed number of steps. It was feared that the motor would mistep and this would eventually lead to antihydrogens missing the active area. As well, the sources are much more localized than the flat stripper foils and thus benefit from precise alignment of the wheel. The $\mu \mathrm{PLC}$ was, in turn, controlled by a digital input/output card. When accessing this ACNET 'device', one could download a new position or read back the current counter level and status. The data written to, and read out through the ACNET system were logged to a file on the ACNET system's VAX. Every 5 minutes a copy was transferred to the E862 workstation via an E835 workstation for monitoring and adding to lookup tables. 


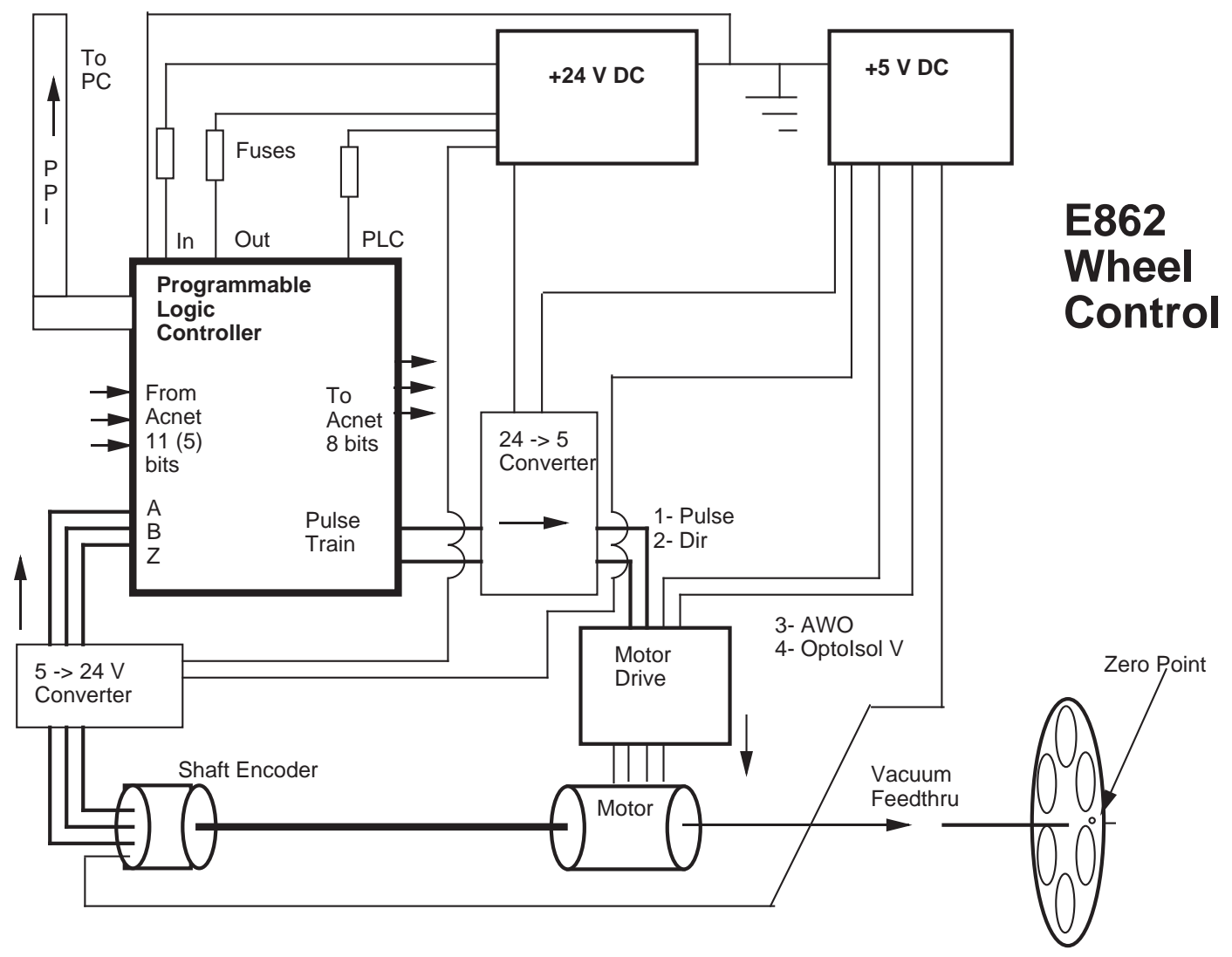

Figure 5.4: Target wheel control subsystem. 


\section{Chapter 6}

\section{Data Analysis}

\subsection{Introduction}

The data files were reconstructed by the offline code and scanned for interesting events. An event was considered interesting if it contained a three-way coincidence in time of the TF1, TF2, and e+ counter signals or alternatively an event with either a hit in PWC \#2 or, lastly, $e^{+}$counter and NaI signals consistent with a positron. The original data files were also subjected to a slower version of the offline code which added information into the event structure about run numbers and beam parameters such as beam momentum, emittances and beam centroid position. The data was analyzed somewhat differently depending on whether the beam momentum was above or below the transition momentum of the accumulator (5196 MeV/c, section 2.1.2). The data contains events taken with the foil target in place and those without the foil target. The event variables used in the event selection are the number of tracks, fractional momentum, vertical residual in PWC \#2, extrapolated track positions at the foil location or alternatively, at the location of the jet, pulse heights (ADC values) and arrival times (TDC values) of the e+ scintillator, both sodium iodide halves, and all four time of flight counter phototubes, and the veto counter arrival times.

The signature for an antihydrogen produced in the jet target is different for foil-in and foil-out data. With the foil in place, the antihydrogen is stripped of the 
$e^{+}$within the foil. Ideally, the $e^{+}$traverses the magnetic spectrometer and hits the middle of the plastic scintillator. It slows down, giving up all its kinetic energy to the scintillator. It then annihilates yielding two back-to-back gamma rays. The gamma rays should have an energy equal to the electron mass but may scatter within the $\mathrm{NaI}$ detector yielding somewhat less. The antiproton will leave a beam momentum track with no indication of a scatter. The track should point back to the jet since it was formed there. When the foil is removed, the antihydrogen will not strip until the vacuum window just before the first PWC is encountered. The $e^{+}$will muliple scatter through almost all of the window with a mean angle which, in most cases, can be seen as a hit in PWC \#1. The antiproton will reconstruct as a beam momentum track.

\subsection{Antiproton beam data}

The data obtained from ACNET (accelerator network) are shown in time plots. The first two figures, 6.1 and 6.2, are histories of the beam angle (in mrad) respectively in the $\mathrm{x}$ and $\mathrm{y}$ dimensions over the course of the fixed target run. They come from measurements of the beam position before and after the gas jet taken every 15 minutes while the data acquisition was running. The $\mathrm{x}$-axis represents an entry for each measurement. The second set of two figures, 6.3 and 6.4 , show the emittances in both dimensions plotted similarly. Finally, figures 6.5 and 6.6 show the antiproton beam centroid position extrapolated to the location of the foil target as a function of (real) time. These beam parameters are used in chapter 7 to calculate geometrical acceptances. 


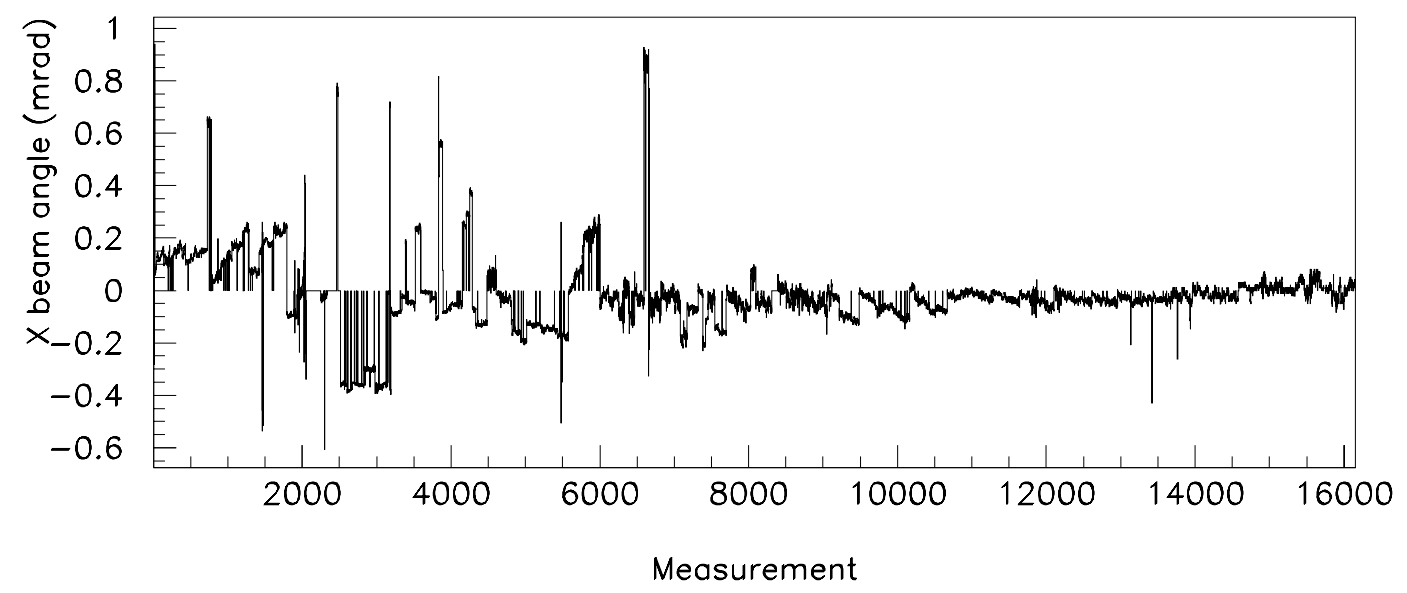

Figure 6.1: Horizontal angle during the run. The $\mathrm{x}$ axis represents one entry for each measurement taken during running. The range is the entire fixed target run.

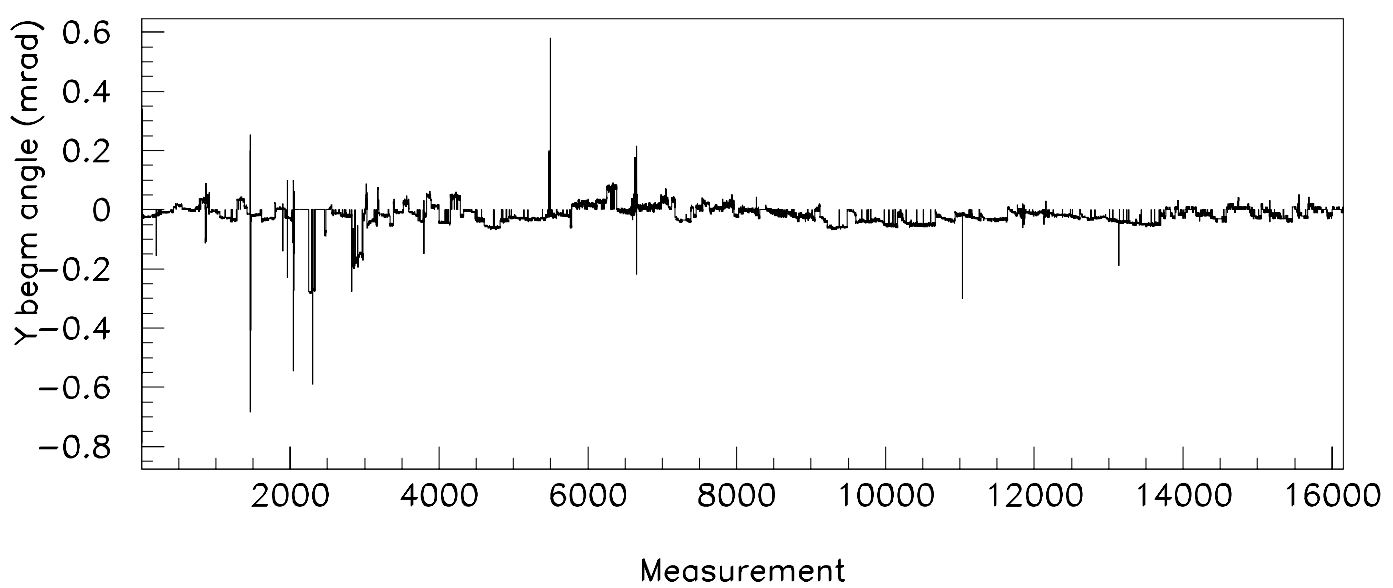

Figure 6.2: Vertical angle during the run. The $\mathrm{x}$ axis represents one entry for each measurement taken during running. The range is the entire fixed target run. 


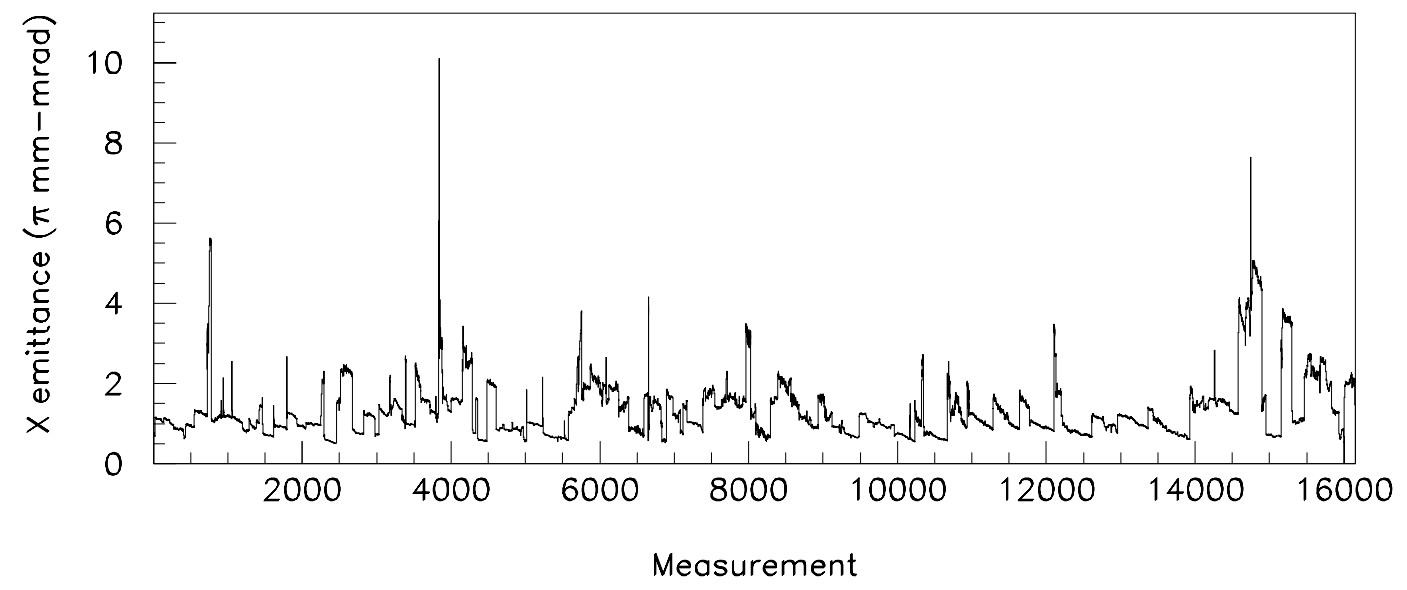

Figure 6.3: Horizontal emittance during the run. The $\mathrm{x}$ axis represents one entry for each measurement taken during running. The range is the entire fixed target run.

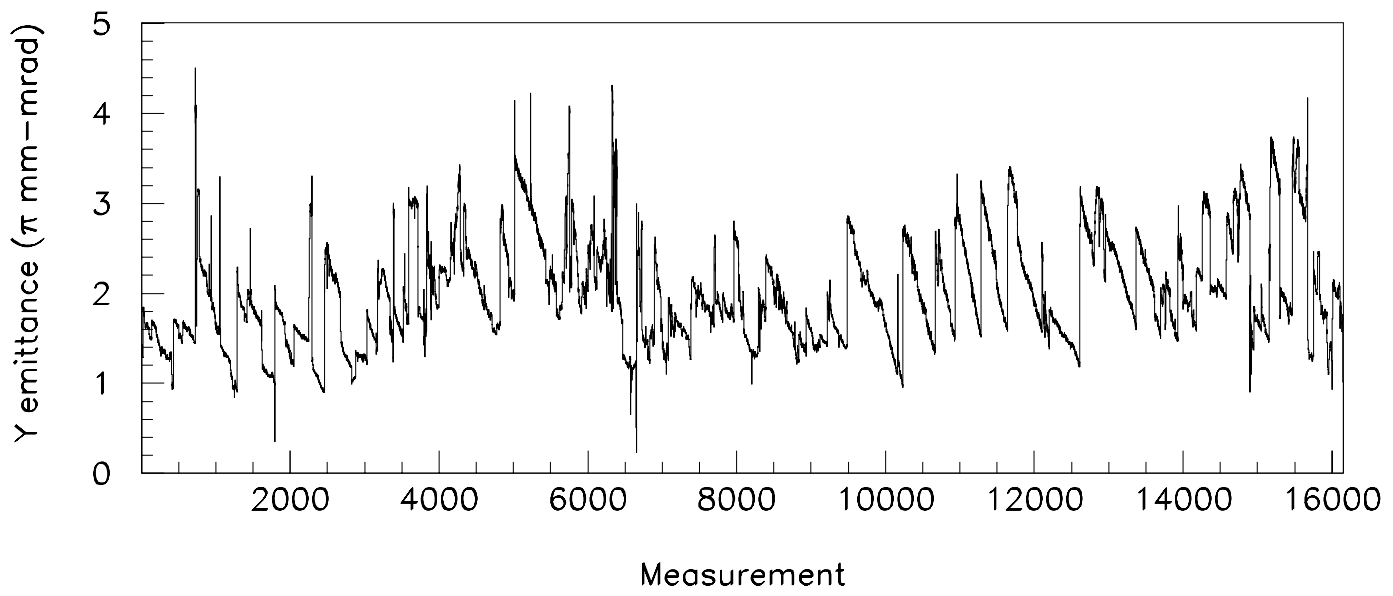

Figure 6.4: Vertical emittance during the run. The $\mathrm{x}$ axis represents one entry for each measurement taken during running. The range is the entire fixed target run. 
BPM derived beam positions at foil
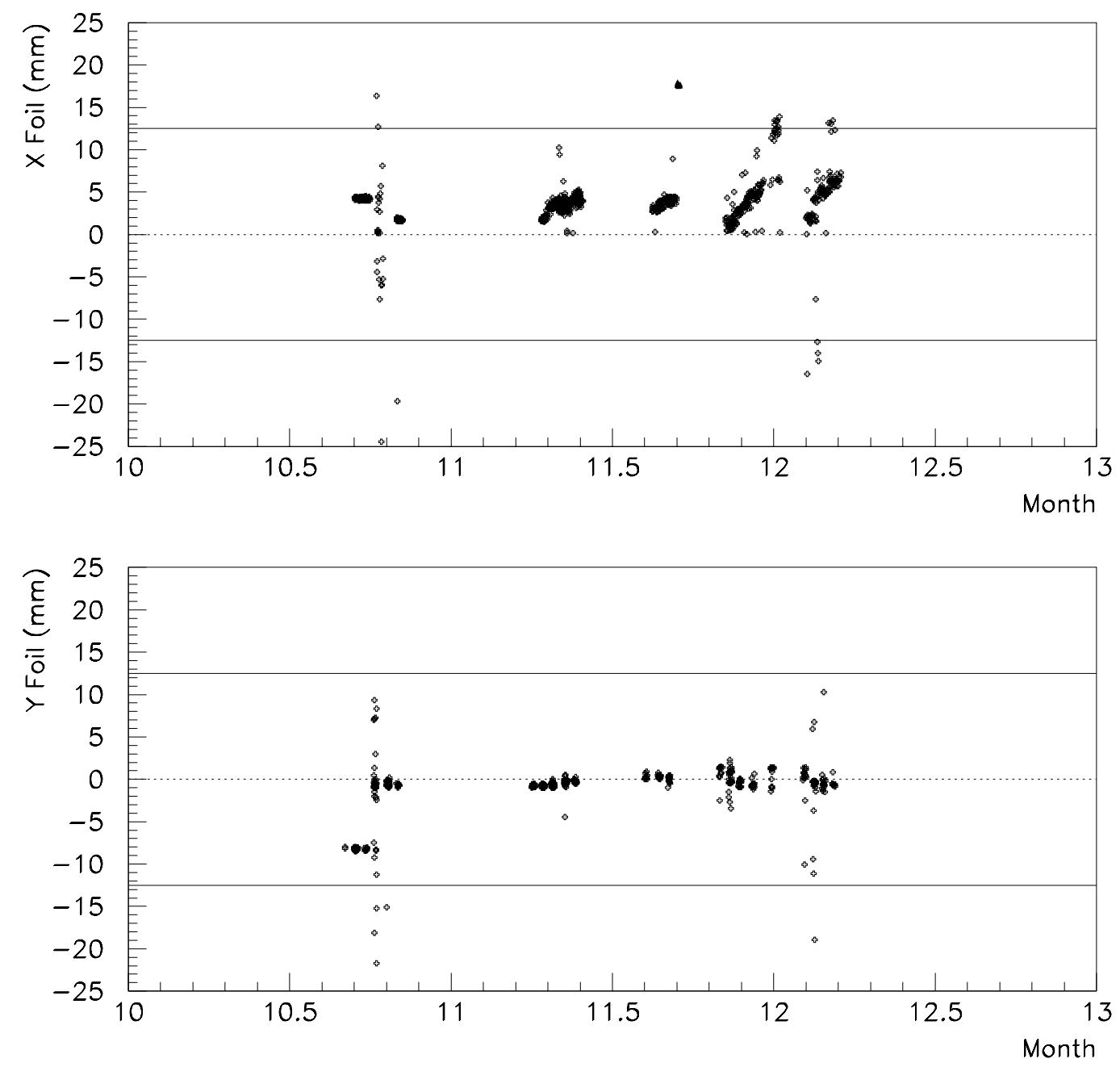

Figure 6.5: Beam centroid positions at foil extrapolated from beam position monitors 8 meters upstream and downstream of jet (1996 data). 

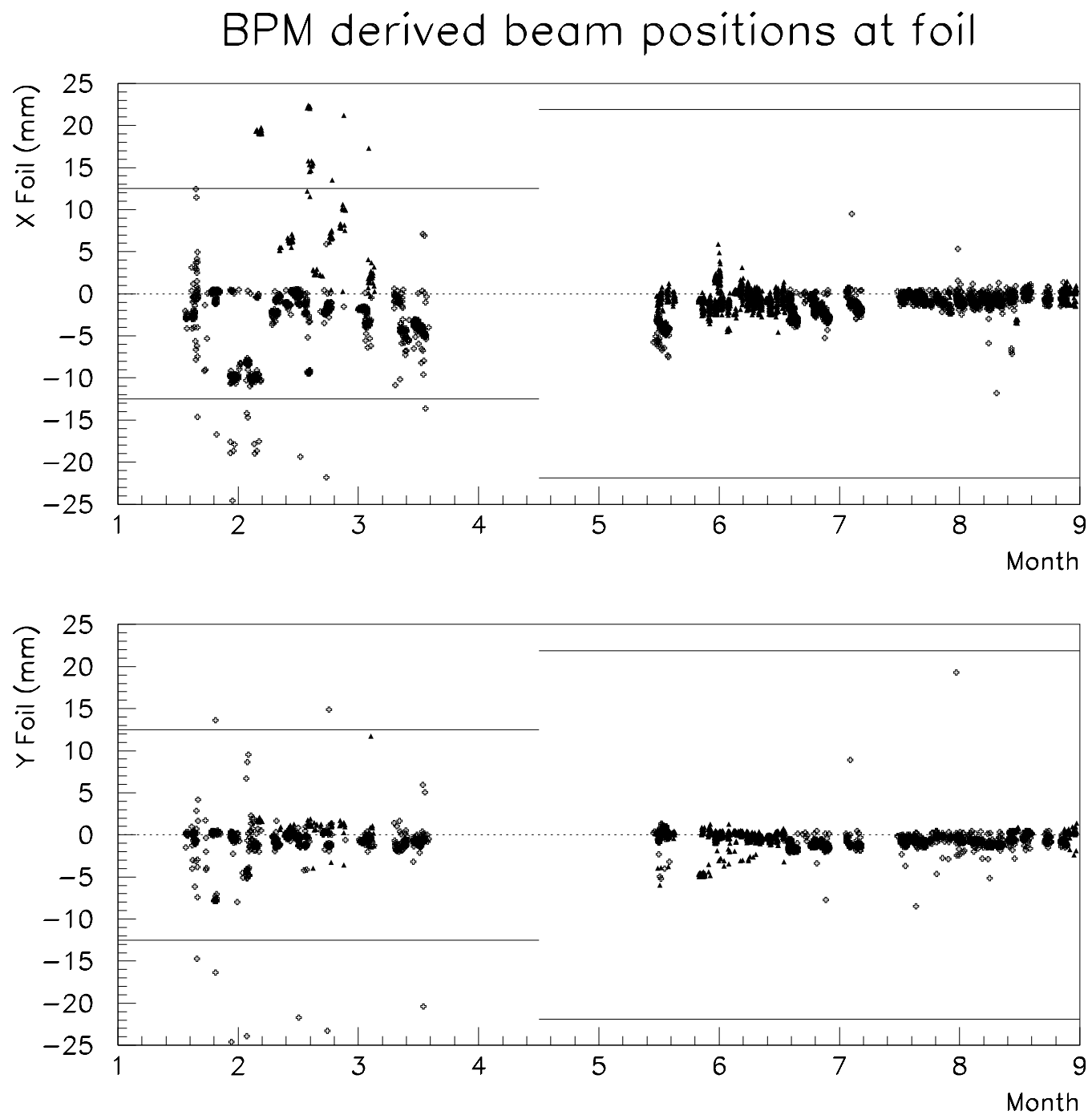

Figure 6.6: Beam centroid positions at foil extrapolated from beam position monitors 8 meters upstream and downstream of jet (1997 data). 


\subsection{Track Reconstruction}

The TDC information could have been used to operate the wire chambers, as done with drift chambers, by weighting positions by hit times to get a resolution $\sigma_{x}<100 \mu \mathrm{m}$. In practice, demanding a window of $\pm 40 \mathrm{nsec}$ around the mean time for a particular chamber proved to be adequate. This eliminated noisy wires which had random hit times. The distribution of reconstructed track positions in the chambers for antihydrogen candidates showed a tight cluster of positions. Even so, the hits in each event were separated by at least a few wire spacings, so using the times to tell the difference was not needed. The differences between the mean times of the three wire chambers were determined, by using delay cables, to be about $100 \mathrm{nsec}$. The resolution for a position measurement was determined by the wire spacing $(1 \mathrm{~mm})$. If two hits were on adjacent wires within $40 \mathrm{nsec}$ of each other, they were considered one particle (one hit) and the hit position was considered halfway between the wires. The width of the arrival time distribution of electrons over different wires is about 20 nsec wide. At least one hit in each of two different planes of a chamber is needed to record a space point. For events with 3 or 4 planes with hits, all hits were used. A space point was calculated from two of them and the third had to be close in both transverse dimensions (to within 1.5 wires) in order to qualify it as a true point. With four planes hit, two points were made from two pairings and compared until one pair of pairs were close in space. After run 20, there were no chambers with 4 planes and a significant fraction of events had only 2 planes of a chamber on. Thus many events had a space point which was not well constrained. Occasionally, groups of adjacent wires were on due to either a high energy particle knocking loose a delta ray or simply from crosstalk in the discriminators.

All possible tracks were ascertained from the space points. For example, if only one chamber had two space points and the other two chambers had only one each, 


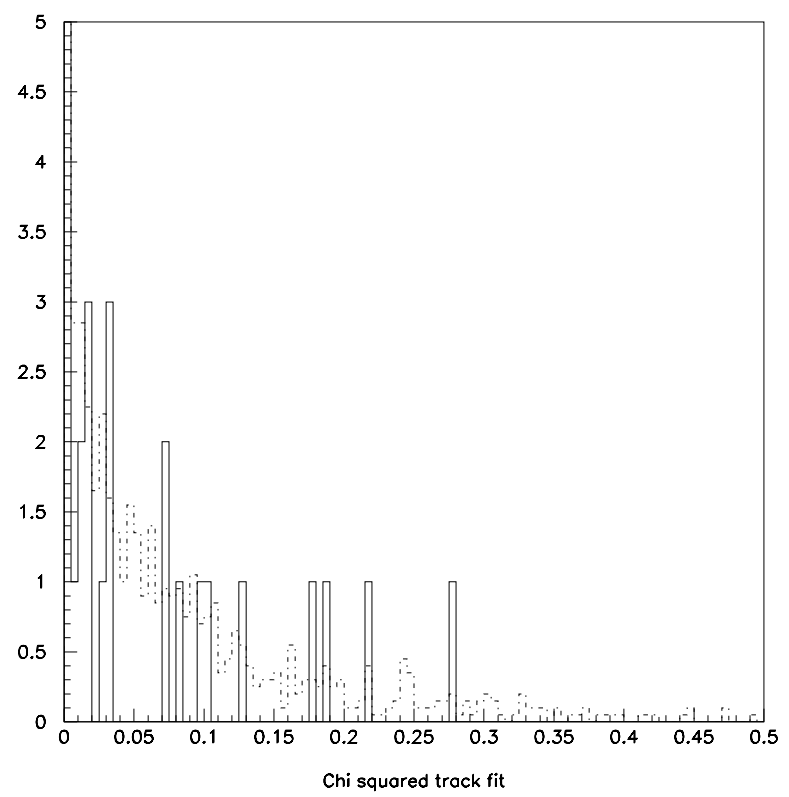

Figure 6.7: $\chi^{2}$ distribution from fits to space points in PWC \#1 for candidates (solid) and all track events (scaled, dash-dotted).

two tracks were usually formed. The quality of the fits to the space points making up the track was characterized by a $\chi^{2}$ variable, (see figure 6.7).

A maximum of 15 tracks were found per event by the offline code. In practice, less than 4 tracks were found per event except when the circulating beam started to hit apertures and created a spray near PWC \#1, (see figure 6.8). The difference between the measured $\mathrm{x}$ position in PWC \#2 and that expected from the measured $\mathrm{x}$ positions in $\mathrm{PWC} \# 1$ and $\mathrm{PWC} \# 3$, corresponding to their best space points, was called the X-residual, (see figure 6.9), and is related to the track momentum by:

$$
X_{\text {res }}=\text { Const } * p_{\text {central }} *\left(\frac{1}{p}-\frac{1}{p_{\text {central }}}\right)
$$

The constant, Const $=49.340$ inch was determined by a fit to the data.

The specific manner in which $X_{\text {res }}$ is calculated is as follows: A straight line is drawn between the hits in PWC \#1 and PWC \#3 in the coordinate system where the central trajectory constitutes the longitudinal axis, $(\mathrm{z})$. The deviation of the 


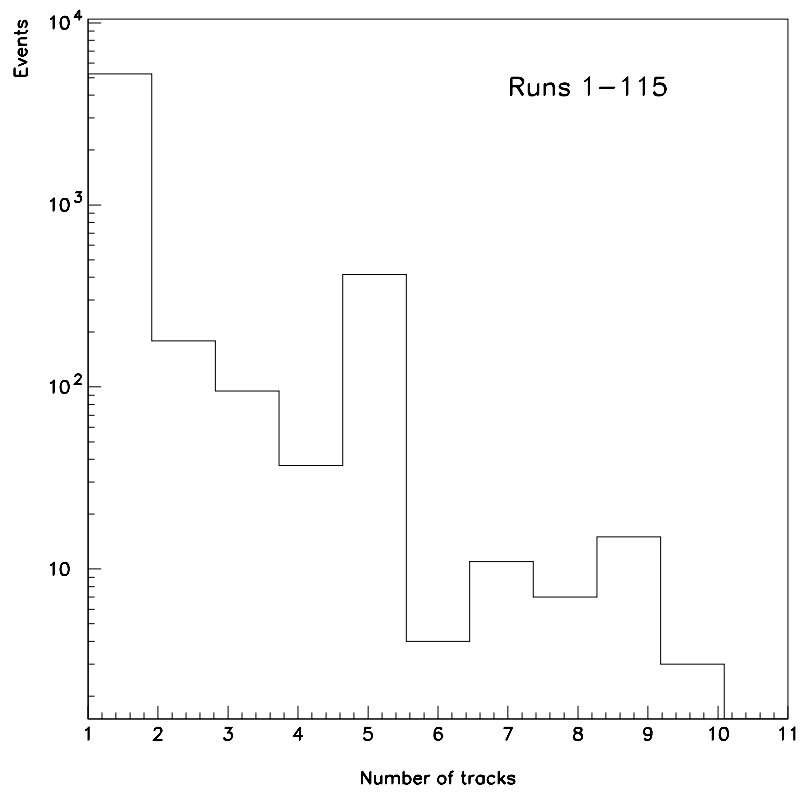

Figure 6.8: Number of tracks per event

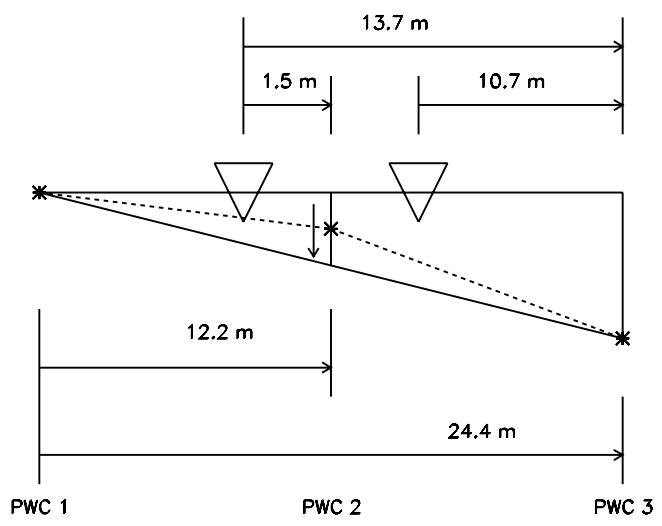

Figure 6.9: Diagram showing how the residual in the bend plane is measured. 


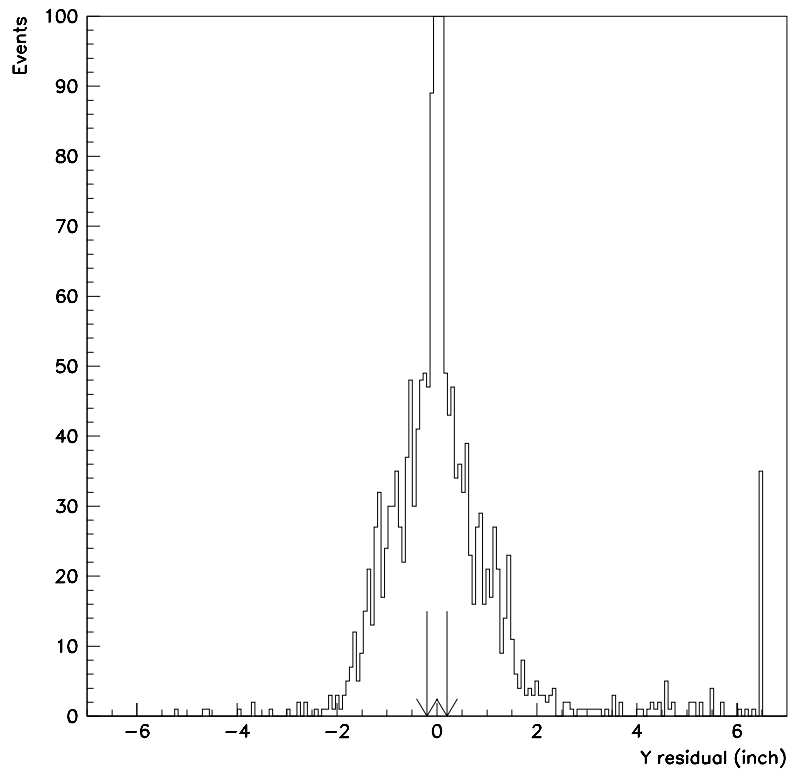

Figure 6.10: Vertical (y) track residuals at PWC \#2 for all events with one track. Arrows show cut based on wire chamber resolution.

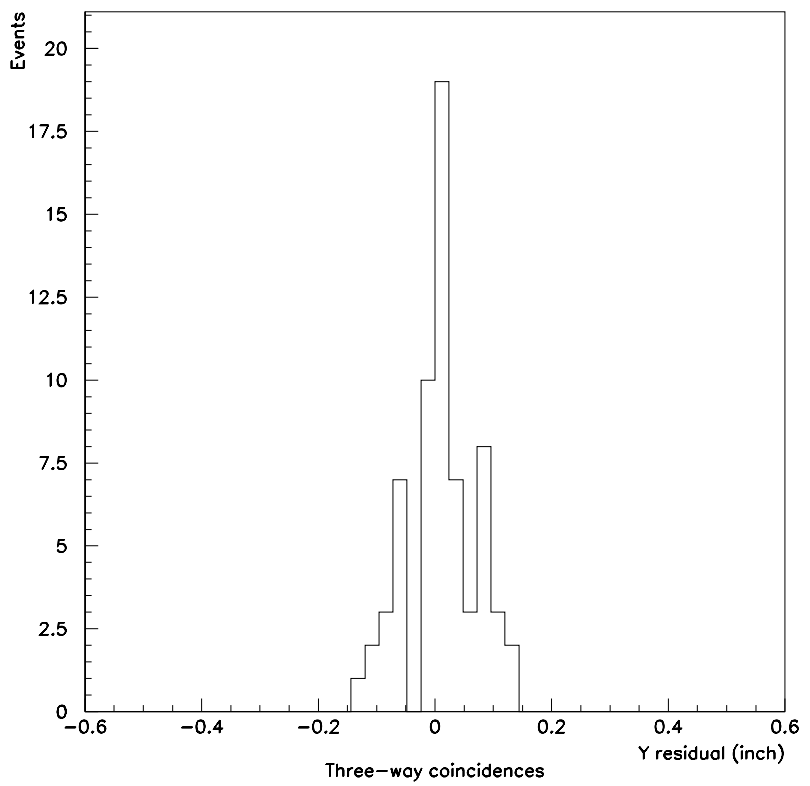

Figure 6.11: Vertical (y) track residuals at PWC \#2 for triple coincidence events. 
hit's $\mathrm{x}$ position in PWC \#2 from where the straight line predicts it should be is denoted as $X_{\text {res }}$, the X-residual. The corresponding Y-residual in PWC \#2, $X_{\text {res }}$, is also calculated from the above method and should be equal to zero, in principle. The cut on the Y-residual is established by the projection of the wire spacing in the vertical and thus a $\left|Y_{\text {res }}\right|<0.15$ inch was used. The triple coincidence events have a Y-residual distribution contained within $\left|Y_{\text {res }}\right|<0.15$ inch, (see figure 6.11). The tracks in general, however, have a gaussian $Y_{\text {res }}$ distribution with a much larger width $(\sigma=0.8 \mathrm{inch}$ ), as seen in figure 6.10. The events falling outside of this cut are mostly tracks that have undegone a scatter near the large antiproton bend magnets. For the candidate events, a cut of $\left|Y_{\text {res }}\right|<0.15$ has been adopted to keep only good tracks.

In fact, since we wanted to accept as many events with tracks as possible, we accepted events in which one chamber had only one plane hit if the others had at least two each. These tracks were classified as a separate type since the PWC \#2 residual in the non bend plane was assumed to be zero to make a straight line. There was no way to cut such an event out if there was a scatter in that direction. The bend plane $X_{\text {res }}$ measurement in PWC \#2 (used for the momentum) relied on horizontal resolution which is better than the more vertical resolution by at least a factor of two. This is because of the stereo angles of the chamber $\left( \pm 7^{\circ}\right)$. The momentum was better measured when $>2$ planes were hit in $\mathrm{PWC} \# 2$.

The exact operating current of the antiproton bend magnets had to be determined experimentally. These magnets derive their operating currents from the accumulator bend bus (A:IB), as do all the dipoles of the ring, and used $50 \mathrm{~mA}$ shunts to lower the current, by a maximum of $7 \%$. The first antihydrogen candidate was found with fractional momentum (0.990), significantly different from 1.000, and having missed the TF2 counter. The fractional momentum is the ratio of reconstructed track momentum to the beam momentum. After this run, the downstream 


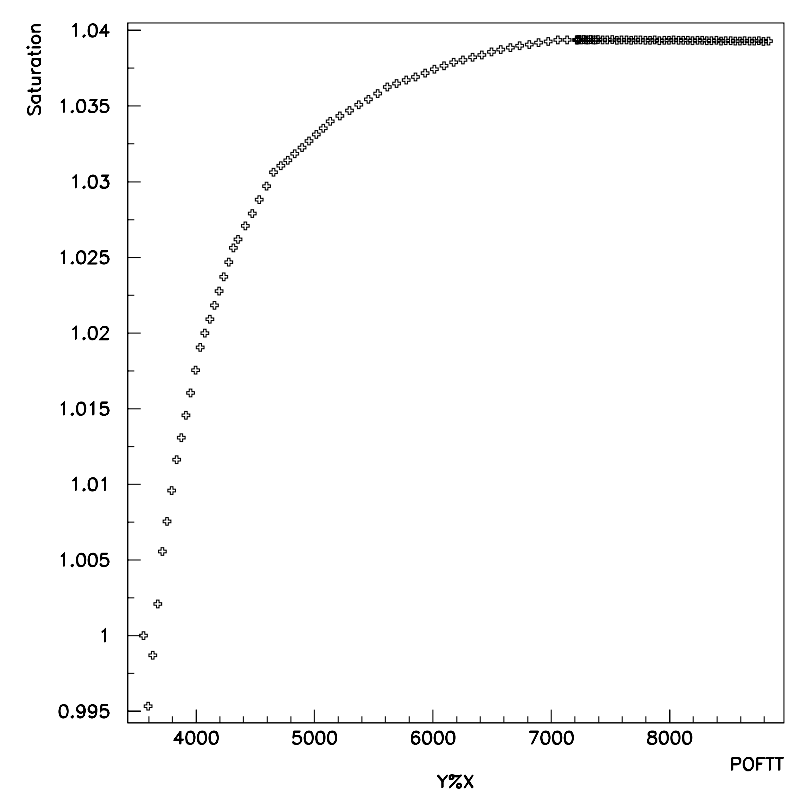

Figure 6.12: Saturation of magnets during deceleration ramps. The variable POFTT is the momentum of particles $(\mathrm{MeV} / \mathrm{c})$ which the magnets are supposed to bend. The $y$ axis is represents the ratio of applied current to magnetic field (normalized) and exhibits large hysteresis as the momentum is increased towards that of the injection.

bend magnet's shunt was adjusted to have a current $2 \%$ of the bend bus current. This bent any subsequent tracks of the same momentum by $1 / 2$ " back onto the center of the counter and the tracks registered a fractional momentum of 1.000. The ideal shunt current was not known exactly because after deceleration the bend bus does not always correspond exactly to the momentum. One reason is that the accumulator dipole shunts are adjusted to get a certain geometry orbit and can be manipulated without changing the overall energy of the beam. In addition, the accumulator magnets were designed to operate well into the saturation regime at the injection energy and a significant amount of hysteresis affected the magnetic fields at the lower beam energies. The latter is technically a nonlinearity which could have been corrected for, in principle. The second is much harder to correct for though. 

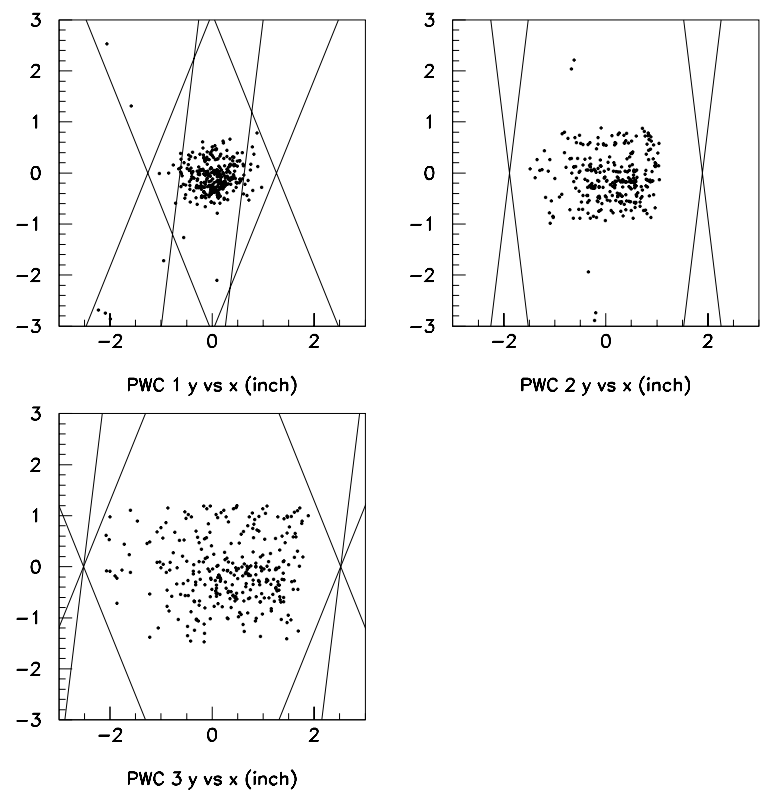

Figure 6.13: PWC profiles of all tracks with $\left|Y_{\text {res }}\right|<0.15$. Noisy wires can be seen in PWC \#2. Wires that define the edges of each plane are shown.

The profiles of the tracks on the wire chambers are shown in figures $6.13,6.14$, 6.15. The wire configurations for runs 1-9 are different from those for runs after 9 . Since only runs 6 - 9 correspond to data when the momentum scale was understood well, these are not shown. The outermost wire of each plane instrumented is shown. The figures correspond to (runs 10-115) all events with good tracks, high momentum $\left(0.997<\frac{p}{p_{\text {beam }}}<1.002\right)$ events without evidence of a $e^{+}$, and triple coincidence events, respectively. 

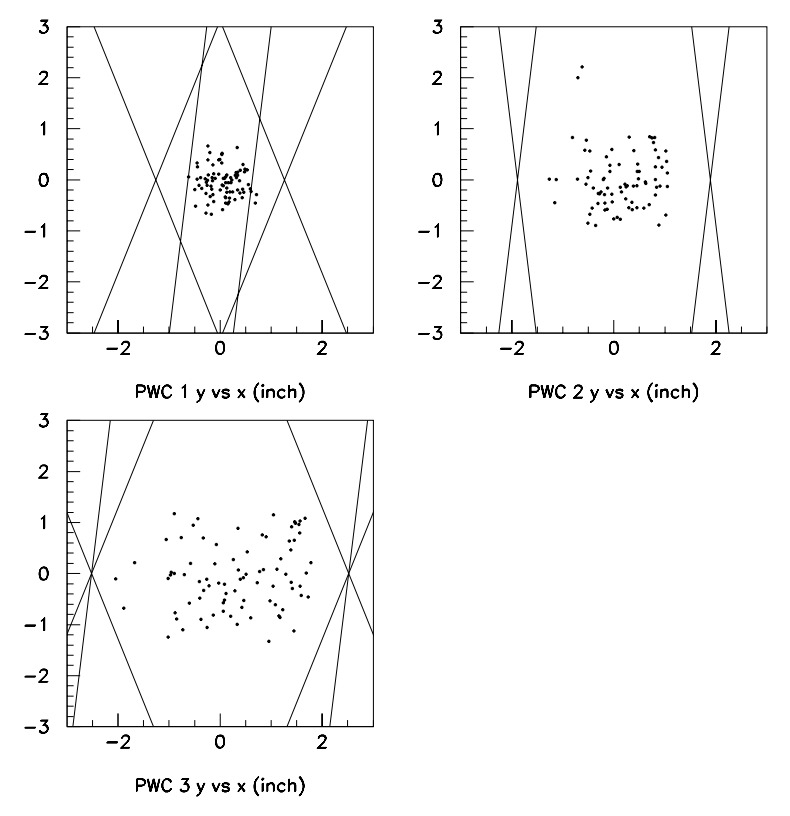

Figure 6.14: PWC profiles of (non 3-way) high momentum tracks with $\left|Y_{\text {res }}\right|<0.15$. Noisy wires can be seen in PWC \#2. Wires that define the edges of each plane are shown.
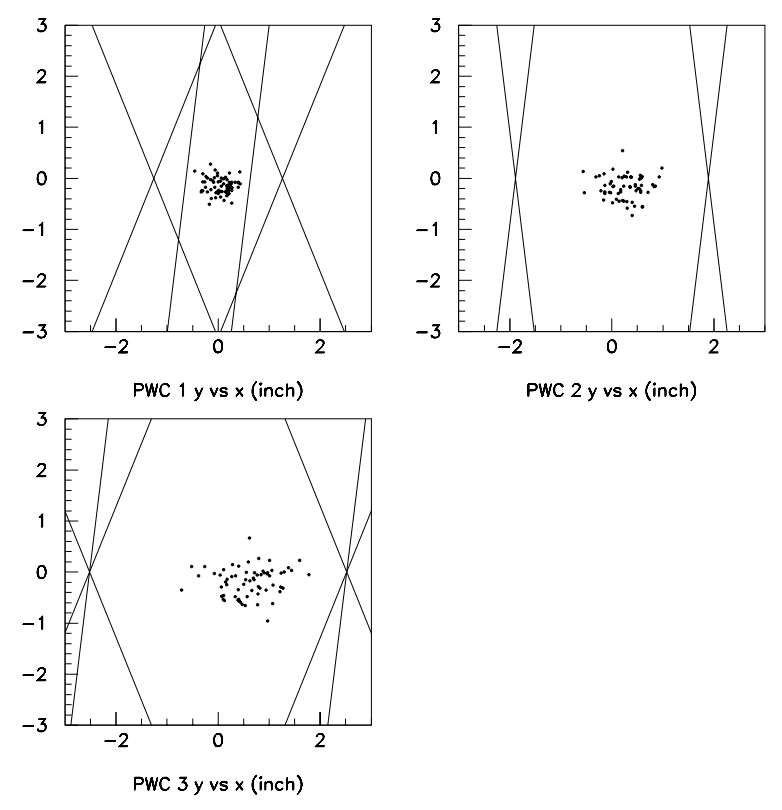

Figure 6.15: PWC Profiles of tracks from three-way coincidence events with $\left|Y_{\text {res }}\right|<$ 0.15 . 


\subsection{Antiproton Background Tracks}

A quick calculation of the momentum acceptance of the spectrometer may be obtained by recognizing that the magnets are very close to chamber 2 so the instrumented region in PWC \#3 defines the momentum acceptance. Antiprotons below $96.15 \%$ of beam momentum (and above 101.9\%) are not registered as tracks. There are events in the data as far out as $95.35 \%$ and $102.75 \%$ (94.25\% and $106.4 \%$ for poorly fit tracks). The fractional momentum distribution shows a peak at $100 \%$ and two larger peaks. Events with beam momentum above transition $\left(p_{\bar{p}}>5.2 \mathrm{GeV} / \mathrm{c}\right)$ have a peak at about $97.5 \%$ of beam momentum while those below transition have a peak at about $98.4 \%$ of beam momentum, (see figures 6.17, and 6.18). Figure 6.22 shows the fractional momentum distribution for antihydrogen candidates which are included in the previous two figures. All the antihydrogen candidates have fractional momentum $>99.6 \%$, except those which did not have a well-defined track and the first candidate event $\left(P / P_{\text {beam }}=0.990\right)$.

Understanding the background tracks is more important for the below transition data since significantly more high momentum $\left(P / P_{\text {beam }}>0.995\right)$ tracks were recorded. A linear dependence exists, (see figure 6.20), for the low momentum, background peak if fractional momentum is plotted versus beam momentum. Relaxing the $\left|Y_{\text {res }}\right|$ requirement allowed enough data to prove the acceptance of the antiproton momentum measurement apparatus was a function only of fractional momentum and not of the beam momentum. This can be seen in figure 6.20 where data fills the region between fractional momenta of $94.25 \%$ and $106.4 \%$.

The secondary peaks in the fractional momentum distribution suggested the antiprotons are scattering off something. An antiproton could scatter by about $-5^{\circ}$ (towards the outside of the ring) just before A5B3. It would then be bent $5^{\circ}$ back to 


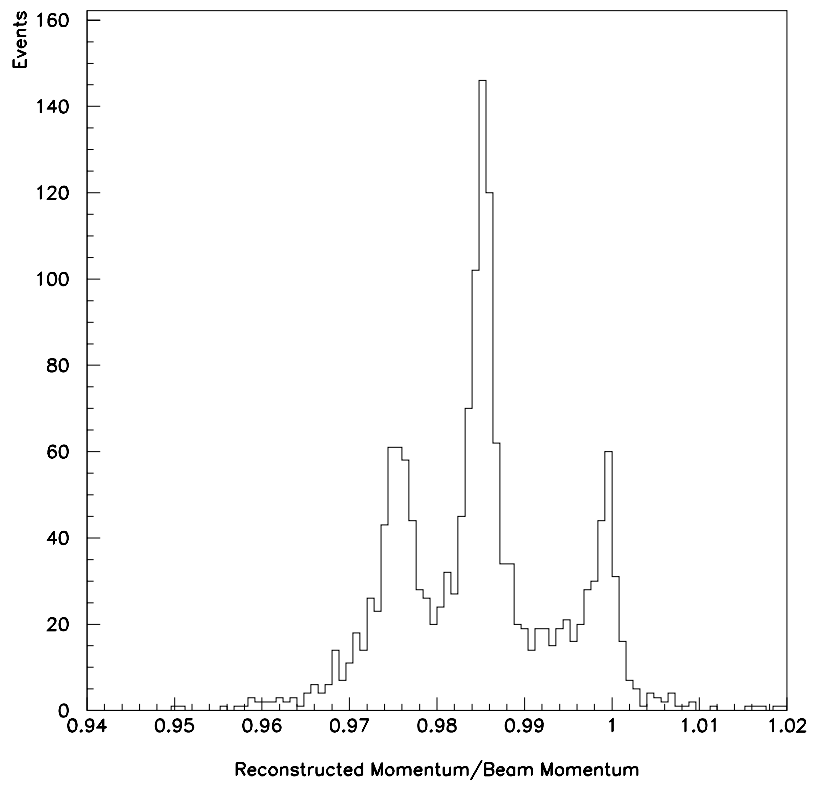

Figure 6.16: Track/beam momentum for foil-in events in runs 1-115.

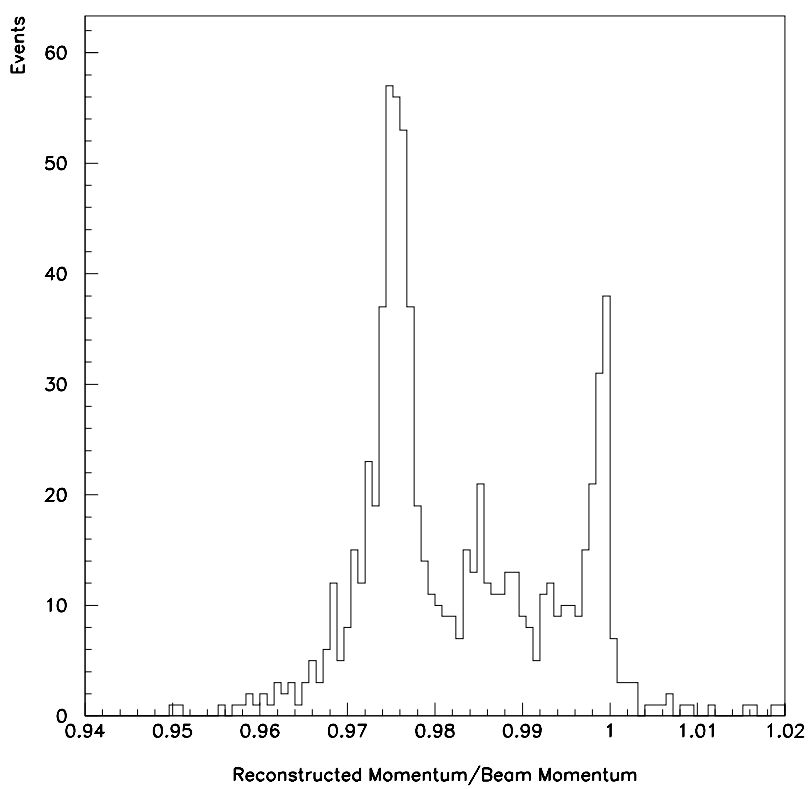

Figure 6.17: Track/beam momentum for foil-in events in runs 1-115 above transition energy. 


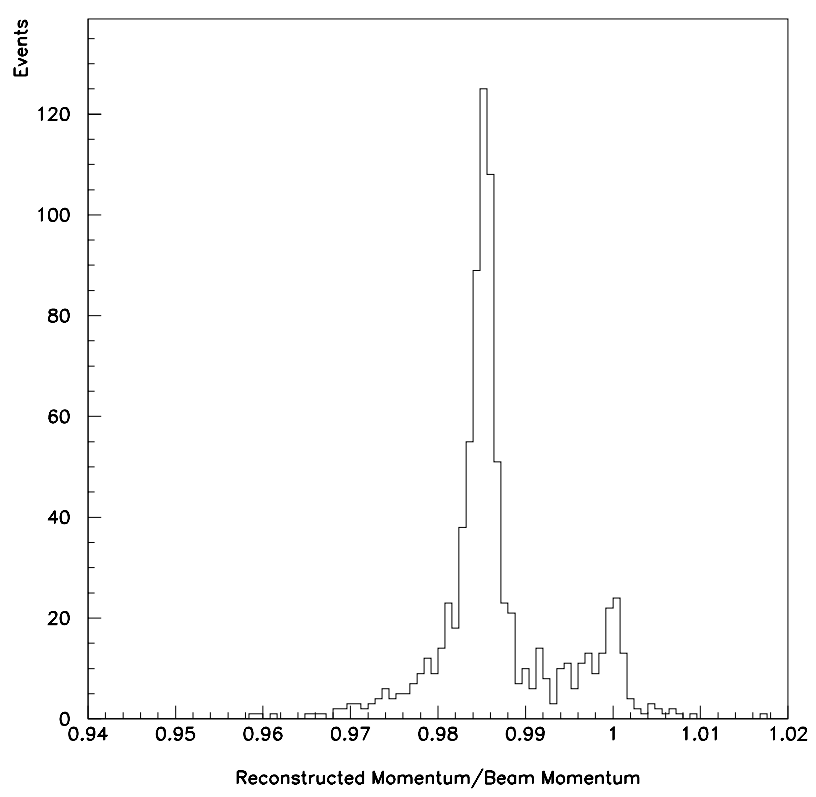

Figure 6.18: Track/beam momentum for foil-in events in runs 1-115 below transition energy.

a centered trajectory pointing down the E862 beamline while traversing the magnet. In doing so, some fractional (longitudinal) momentum is lost. The only explanation which matches is elastic scattering off single protons (hydrogen gas). It is reasonable for a small amount of hydrogen gas to travel far from the jet. Figure 6.19 shows a diagram of the elastic scattering. Figure 6.21 shows that the result for scattering off of helium atoms is far from the observed background peaks. It is clear that for larger target atomic numbers, the momentum loss is smaller by $Z^{2}$. Also shown is the effect of a $10 \%$ error in the $\int B d L$ of the magnet. The A5B3 magnet has a bend angle of 0.0862 radian [67]. Extrapolating these tracks back to the area near A5B3 shows they intersect the beampipe no more than 18" upstream of the beginning of A5B3 if the $-5^{\circ}$ scatter is not included. This indicates the elastic scatter took place within $18^{\prime \prime}$ of the upper edge of the magnet steel and not closer to the jet.

The amount of background gas in the vicinity of the A5B3 magnet can be surmised from the number of tracks and the cross section for the scattering process. 


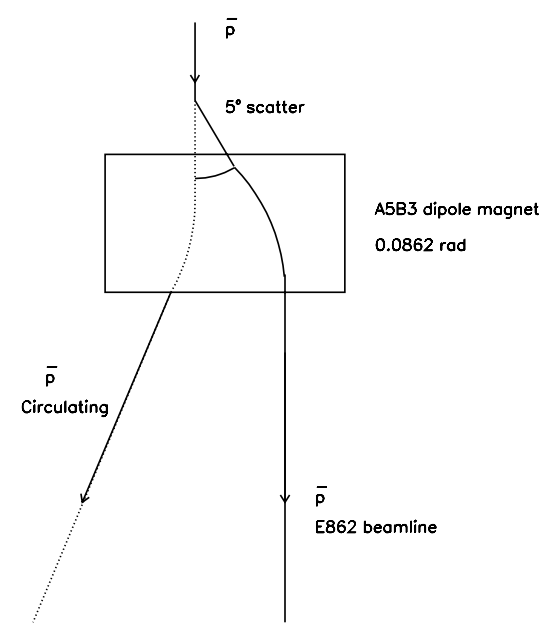

Figure 6.19: Diagram of elastic scattering

The Rutherford scattering cross section is $(Z=1)$ for hydrogen[54]:

$$
d \sigma / d \Omega=\frac{Z^{2} \alpha^{2}\left(F\left(q^{2}\right)\right)^{2}}{4 p^{2} \sin ^{4}(\theta / 2)}
$$

Let's assume the proton form factor is equal to one. This can be integrated around the $5^{\circ}$ scatter needed to cancel the bend from the magnet. The angular range is determined by the antiproton spectrometer's smallest aperture in angle drawn to A5B3. The half aperture of PWC \#3 is at most 2.5". Thus $\delta \theta=1.8 \mathrm{mrad}$. The cross section is $\sigma=0.0072 / p^{2}$ or $76 \mathrm{nb}$ for $6 \mathrm{GeV} / \mathrm{c}$. The effective integrated luminosity for 1559 background tracks is now $20.5 \mathrm{nb}^{-1}$. If this is compared to the instantaneous luminosity and the total luminosity integrated at the jet, the instantaneous luminosity for the background scatter process is found to be $\mathcal{L}=4.7 \times 10^{27} \mathrm{~cm}^{-2} \mathrm{sec}^{-1}$. Using an average figure for the number of antiprotons and revolution frequency, the background gas density is:

$$
\rho_{b k g}=1.8 \times 10^{10} \text { atoms } / \mathrm{cm}^{2}
$$

In order to get a density 2000 times smaller than the jet density, it is assumed that the $5 \%$ of gas escaping from the region of the jet diffuses down the beampipe with an 


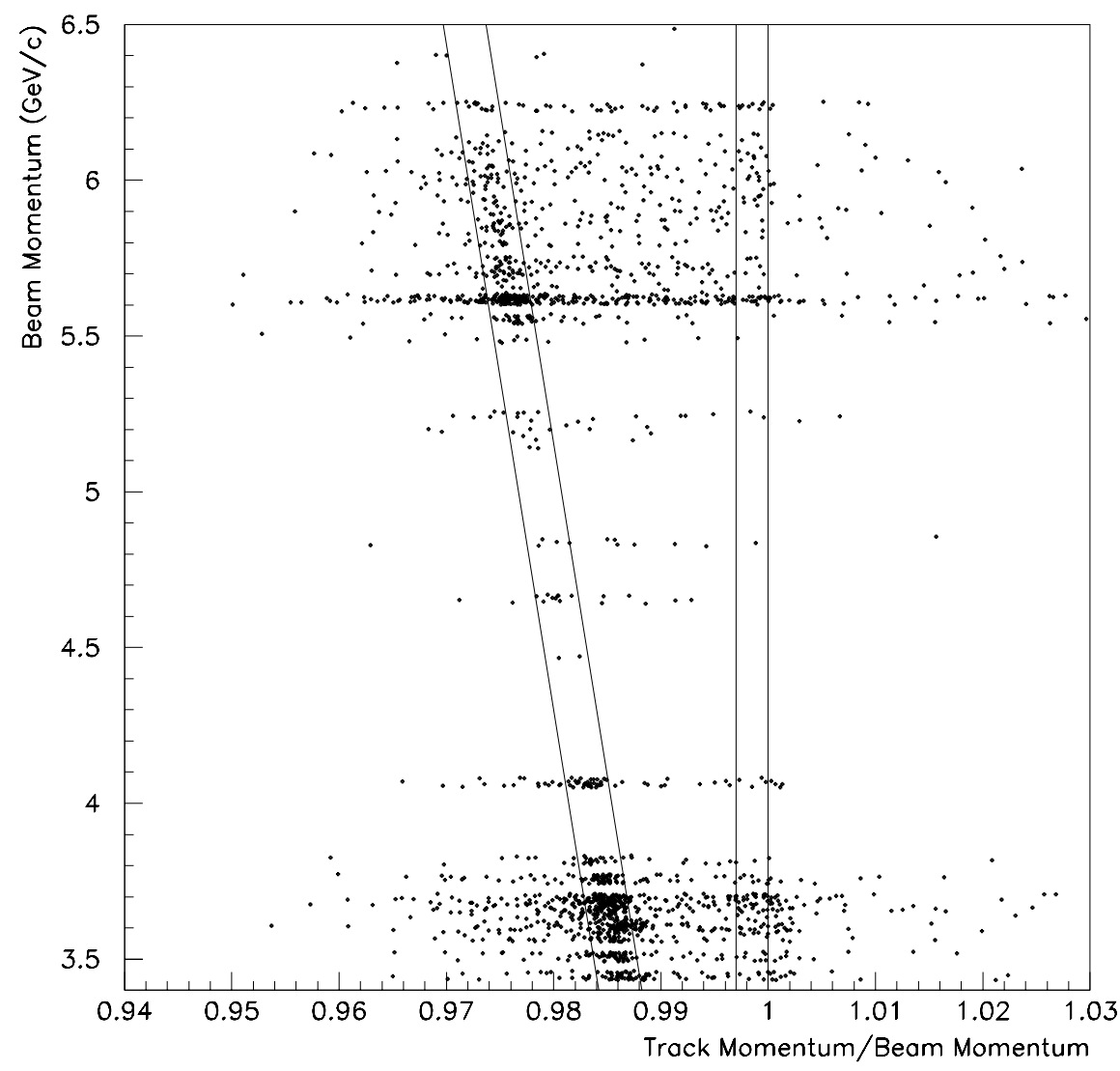

Figure 6.20: Events with one track and $\left|Y_{\text {res }}\right|<0.1$ inch. Background tracks with higher beam momentum have a lower fractional momentum. Lines correspond to expected ranges of large numbers of events.

approximately exponential profile. The above background gas density indicates the $1 /$ e length is about 10 feet.

The curiously large number of $P / P_{\text {beam }} \sim 1.000$ tracks below transition can most likely be attributed to elastic scattering off the aluminum beampipe. This is plausible considering the larger emittance of the beam, below transition. The momentum loss for scattering off of aluminum is no more than $\frac{\delta p}{p}=2 \times 10^{-4}$ at $6 \mathrm{GeV} / \mathrm{c}$ which is indistinguishable from a loss of zero. 


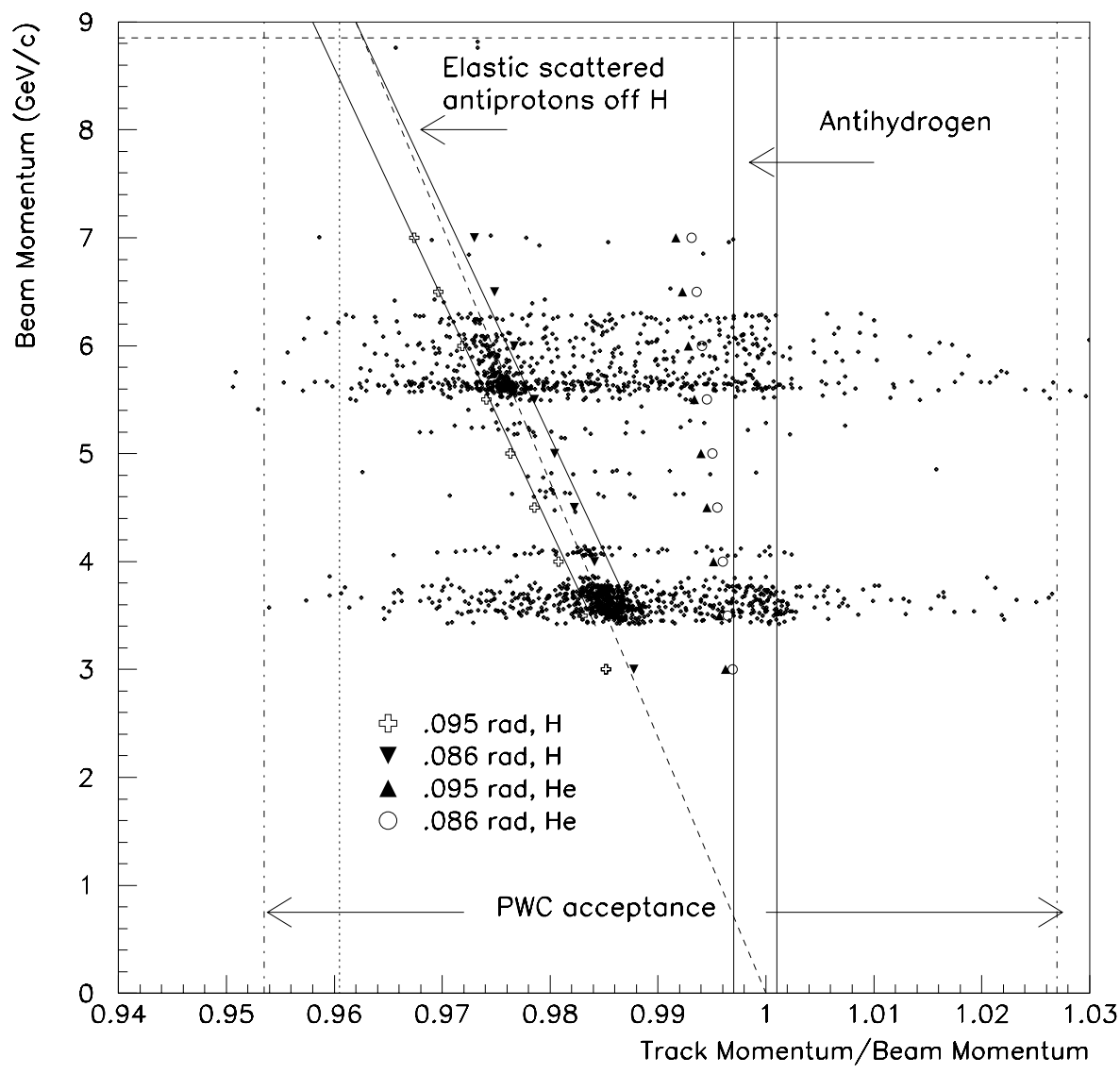

Figure 6.21: Calculated elastic scattering off hydrogen and helium superposed to show how this accounts for the background, low fractional-momentum tracks. Many below transition tracks near beam momentum are thought to be scattering off aluminum beampipe. All events in this plot have only one track. 


\subsection{Secondary Backgrounds}

Various background processes from the beam-jet interaction region have been considered. Since the detector is so far downstream, the small solid angle suppresses the overwhelming majority of possible background channels. The additional demand that one particle be at the beam momentum $(3-9 \mathrm{GeV} / \mathrm{c})$ and the other be three orders of magnitude lower in momentum $(\mathrm{MeV} / \mathrm{c})$ restricts the possible phase space. This suppresses background channels which do not produce a near beam momentum antiproton and a positron.

For example, the charge exchange reaction, $\bar{p} p \rightarrow \bar{n} n$, will yield an antineutron which can either beta decay or charge exchange back to $\bar{p} p$ by hitting an obstruction. The chance it does this near the foil is small. Reference [89] includes $d \sigma / d t$ as a function of $\mathrm{t}$ for various reactions including $\bar{p} p \rightarrow \bar{n} n$ at $7.76 \mathrm{GeV} / \mathrm{c}$ antiproton momentum which is within our beam momentum range. The cross section at small angles is about $2 \mathrm{mb} /(\mathrm{GeV} / \mathrm{c})^{2}$. Let's assume the antineutron passes through the A5B3 bend magnet and interacts within the first wire chamber where the beam spot is only about $20 \mathrm{~cm}^{2}$, (see figure 6.13). At a distance of 28 meters from the jet, the solid angle is, at most, $d \Omega=2.6 \times 10^{-6}$. The chance the $\bar{n}$ interacts in the chamber material is only $10^{-2}$. The number of antineutrons expected, found by integrating $d \sigma / d t$ over $-d t \simeq 4 p^{2} \sin ^{2} \frac{\theta}{2},=\frac{p^{2}}{2 \pi} d \Omega=3.4 \times 10^{-5}$, is $8.8 \times 10^{6} \bar{n}$ using $130 p b^{-1}$ of integrated luminosity. The mean four momentum transfer in the reaction is about $9 \times 10^{-3}$ (well outside the accepted solid angle) since the transverse momentum is on the order of the pion mass $(135 \mathrm{MeV} / \mathrm{c})$. The chance the $\bar{n}$ creates a $\bar{p}$ when it interacts is $4.9 \times 10^{-3}$. This is the ratio of charge exchange total cross section to total inelastic cross section $(58 \mathrm{mb})$. Finally, the antineutron must have the correct angle to make it to the last wire chamber. The chance it does this is $1.1 \times 10^{-3}$ Altogether, the 
number of double charge exchange events which could mock antihydrogen is expected to be only $5 \times 10^{-1}$.

Other backgrounds were considered such as arising from long-lived neutrals produced in the gas jet $[90]$.

- $\bar{p} p \rightarrow K_{L}+X, K_{L} \rightarrow \pi^{-} e^{+} \nu$, or $K_{L} \rightarrow \mu^{-} \bar{\nu}_{\mu} e^{+} \nu_{e}$ :

The kaon has a $6.7 \times 10^{-4}$ chance to decay within $5 \mathrm{~cm}$ of the foil target.

- $\bar{p} p \rightarrow \bar{\Lambda}^{o}+X, \bar{\Lambda}^{o} \rightarrow \bar{p} \pi^{+}:$

although lambdas are attenuated by $2 \times 10^{23}$ over $10 \mathrm{~m}$.

- $\bar{p} p \rightarrow \pi^{o}+X, \pi^{o} \rightarrow \gamma \gamma \rightarrow e^{+} e^{-} e^{+} e^{-}:$

with an unrelated $\bar{p}$.

- $\bar{p} p \rightarrow \bar{n} \rightarrow \bar{p} e^{+} \nu:$

The probability the $\bar{n}$ decays within $5 \mathrm{~cm}$ of the foil target is $4 \times 10^{-4}$, so similar to the double charge exchange process, the number of $\bar{n}$ is less than one.

The hypothesis that an antiproton interacts with the beampipe wall upstream of the A5B3 bend magnet has been explored. If an antiproton traverses $20 \mathrm{~cm}$ of pipe and only has multiple small angle Coulomb scatters, the antiproton only receives a few mrad of bend. If on the other hand, it gets a large elastic scatter such that it can bend by $5^{\circ}$ and travel straight down the neutral beamline, the average loss of longitudinal momentum is a few percent. See section 6.4 for more detail. 


\subsection{Foil Target-In Data}

\subsubsection{Description of Foil-In Data}

Certain running conditions prompted consideration of only the runs where the beam and detector were working properly. The parts of runs where stacking took place were removed from the data set and stored as diagnostic data. Parts of runs 33, 68,85 , and 93 contained stacking periods. For runs 51,76 , and 77, the PWC interface crate was off so that the wire chambers' output was the result of unterminated cables and lots of noise. Run 78 had the PWC discrimator crates off. Run 75 had the $e^{+}$ counter's base inadvertently removed. Runs 1-5 had the shunt of one of the antiproton dipoles set incorrectly. The very first antihydrogen event was used to correct the shunt current as described in section 6.4. In addition, runs 1-3 had incorrect delay cable lengths for the $e^{+}$detector elements. Runs before run 14 had a PWC high voltage setting lower than the nominal $-1400 \mathrm{~V}$ used afterwards. The PWC hit efficiency is a strong function of applied voltage. Runs before run 21 had a different PWC physical configuration. In runs after run 20 , no antihydrogen candidates were seen in the region of PWC \#1 or PWC \#3 which previously had not been instrumented. Finally, for certain runs, the acceptance $\mathrm{x}$ efficiency correction was quite small so these runs are treated with special attention (beam steering acceptance, TF2 inefficiency).

In the foil-in data, 78 candidates were observed. Of these, two had the signature of the foil-out candidate events (high momentum track with spectator hit in PWC \#1) and are discussed in section 6.7. The remaining events had all or part of the triple coincidence signature. Of the 76, eight were not triple coincidences at the trigger level. Seven of these suffered from the problem that one of the two phototubes needed for the coincidence in TF2 ( $T F 21 \otimes T F 22)$, was found to be inefficient towards the later runs. The events were recorded since the $T F 1 \otimes e^{+}$trigger requirement was 
met. Upon examination, all these events showed the other TF2 phototube to be on as well as the existance of a beam momentum track. The TF2 trigger inefficiency is discussed in section 7.1. The eighth was the first antihydrogen candidate whose antiproton track missed the TF2 counter. No events were seen which had a true trigger efficiency problem in the sense that some electronics mistiming prevented one of the three triggers formed from the coincidence of two counters from firing when the other two did. I will call all of these triple coincidences since evidence was seen of a signal either in the trigger or in the ADCs or TDCs from each of the counters TF1, TF2, E+. The one exception to the rule is an event which did not have the $\mathrm{E}+$ counter on but was considered because one of the NaI halves showed a pulse height within the expected range. This event will be discussed as it may be a background.

Of the 76, 65 formed one track. Four did not form a track. Two of these were in run 78 when the PWC discriminator crate was turned off. Another was eliminated because 3 of 4 of the hits in PWC \#1 were too early. There is a $40 \mathrm{nsec}$ time window around the mean time for the chambers' signals. The last event was eliminated because two chambers had only one plane hit. Neither could produce a space point and a track could not be made. Of these 76 events, four have a beam momentum below transition in all of $7.6 \mathrm{pb}^{-1}$. The other 72 are above transition in a total of 77 $\mathrm{pb}^{-1}$

\subsubsection{Foil-In Momentum Distributions}

Dealing with the above transition data first, the distribution of the ratio, reconstructed track momentum divided by beam momentum, for those events having one track is shown in figure 6.22. It is a thin distribution of momenta around the beam momentum. Figures 6.23, and 6.24 show these distributions, for above transition 


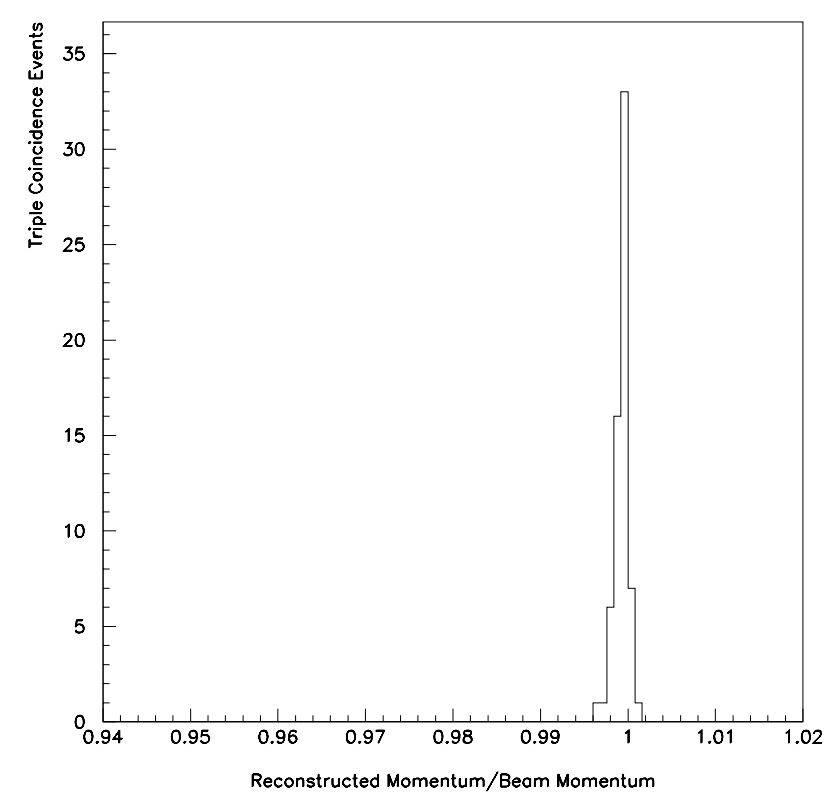

Figure 6.22: Track momentum/beam momentum for triple coincidence candidates. The first event, $\left(\frac{P}{P_{\text {beam }}}=0.990\right)$, is not included.

and below transition respectively, superimposed upon all the data corresponding to foil-in running for the same momentum range. The antihydrogen triple coincidence candidates above transition nearly fill the entire $\frac{P}{P_{\text {beam }}}=1.000$ peak. The ratio of candidates to high momentum track events without an $e^{+}$is about $2: 1$. Below transition, the situation is much worse, at least 30:1. This is thought to be antiprotons scattering off a metal aperture by $5^{\circ}$ and being bent back by A5B3 by $5^{\circ}$ in the opposite direction. The mechanism is explained in section 6.4 for the case of a scatter off hydrogen. For aluminum, the fractional momentum changes by no more than 0.005 so there is no way of presently telling whether these tracks are not antihydrogen with the present setup since the positron may be lost. This is not very plausible given the knowledge of the $e^{+}$spectrometer. They are assumed to be scattered antiprotons. 


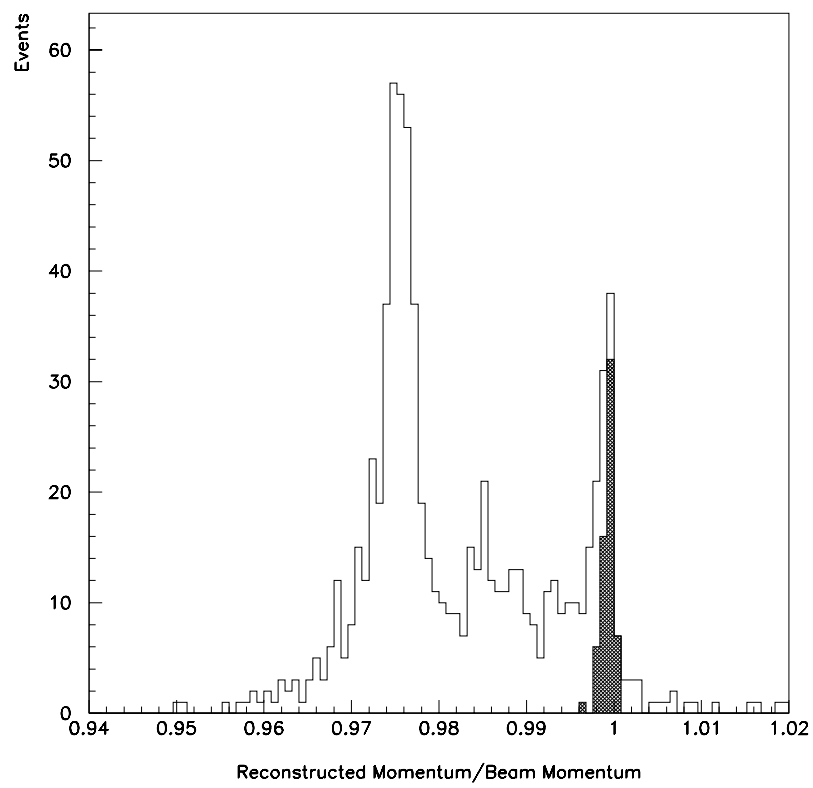

Figure 6.23: Track momentum/beam momentum for triple coincidence events superimposed on a histogram of the same variable for all events. Both are only above transition.

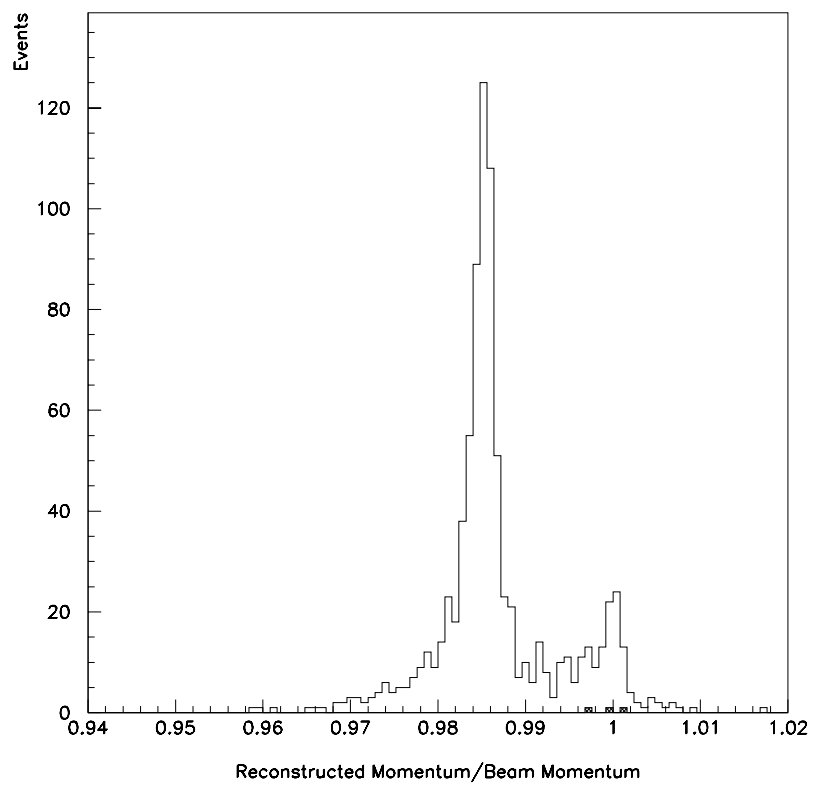

Figure 6.24: Track momentum/beam momentum for triple coincidence events superimposed on a histogram of the same variable for all events. Both are only below transition. 


\subsubsection{Profiles at foil}

The set of tracks including antihydrogens can be extrapolated to the position of the foil as well. Figures $6.25,6.26$, and 6.27 show the track positions at the foil, for all tracks, for triple coincidence events (with tracks), and high momentum events without evidence of a $e^{+}$, respectively. The first plot has the distribution of all tracks peaked to one edge of the foil in the horizontal direction. The position on the foil one would expect from using the positions of the antiproton beam centroid can be calculated as well. The beam moves around quite a bit as can be seen in the plots of beam position versus time, figures 6.5, and 6.6. If the measured candidate event track positions are subtracted from the calculated positions, the resulting distribution yields a measure of the size of the antihydrogen beam at the foil location. Figure 6.28 shows the measured foil positions of candidates after subtracting the centroid positions of the beam obtained from ACNET information. The beam size expected from emittance and flying wire measurements is $\sigma_{x} \sim 0.3^{\prime \prime}$ at the foil. 


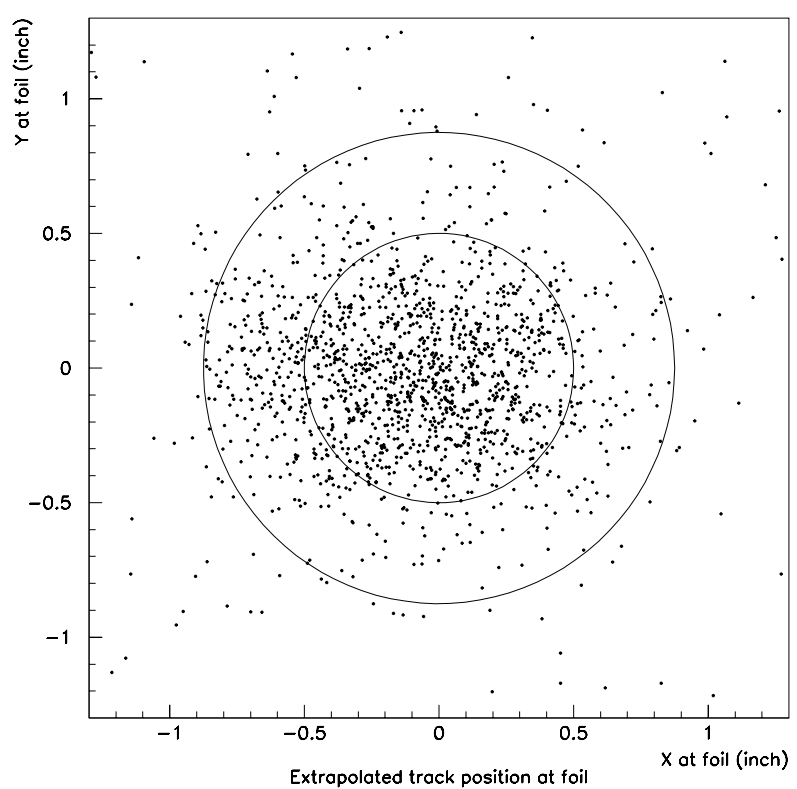

Figure 6.25: Track position of all events with one track at the foil extrapolated from PWC \#1, PWC \#2, PWC \#3.

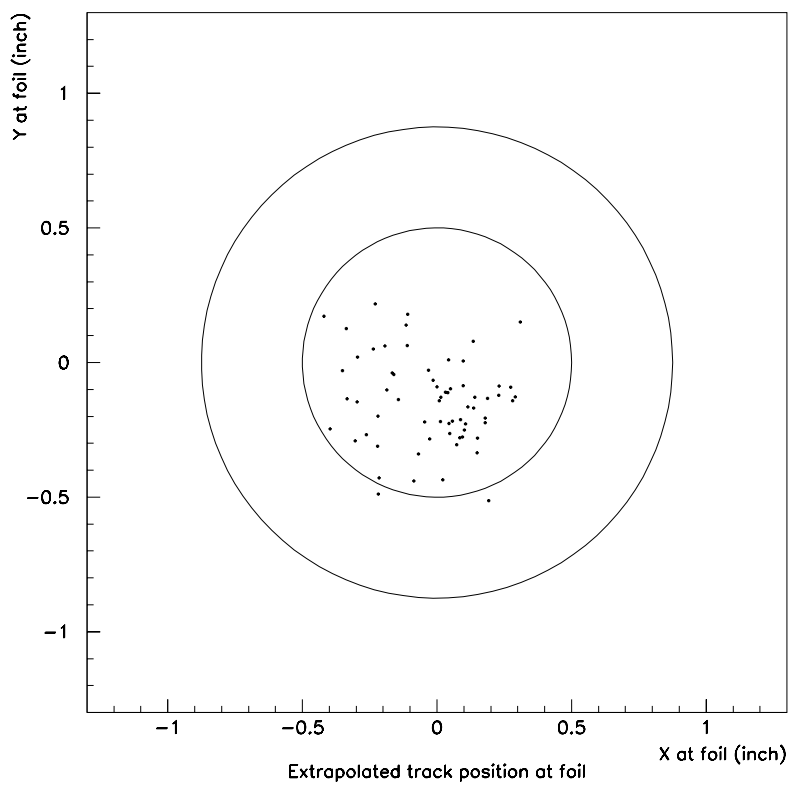

Figure 6.26: Track position of three way coincidence events at the foil extrapolated from PWC \#1, PWC \#2, PWC \#3. 


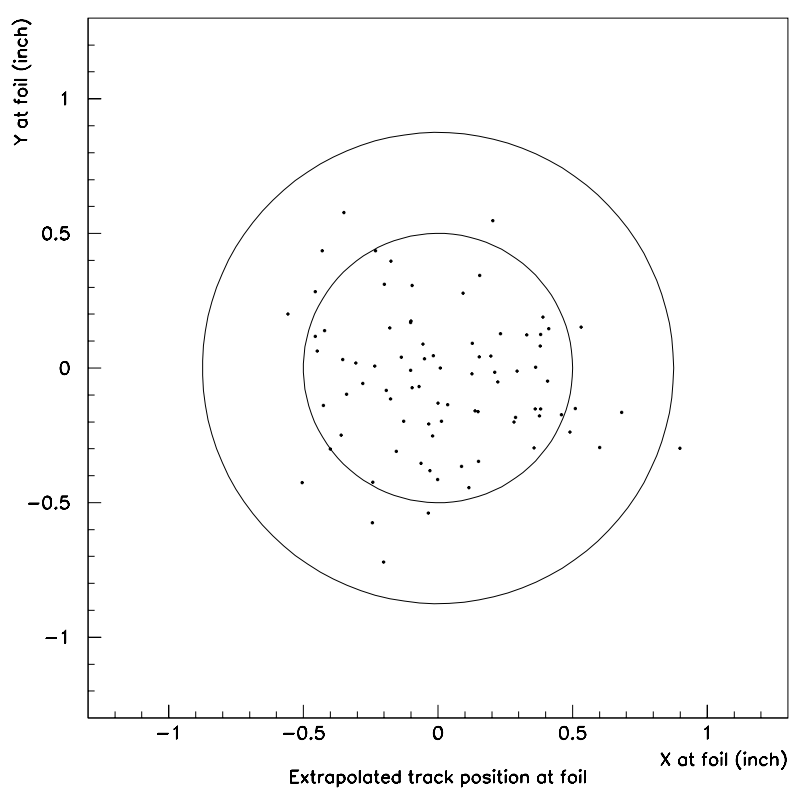

Figure 6.27: Track position of high momentum events without an associated $e^{+}$hit at the foil extrapolated from PWC \#1, PWC \#2, PWC \#3.

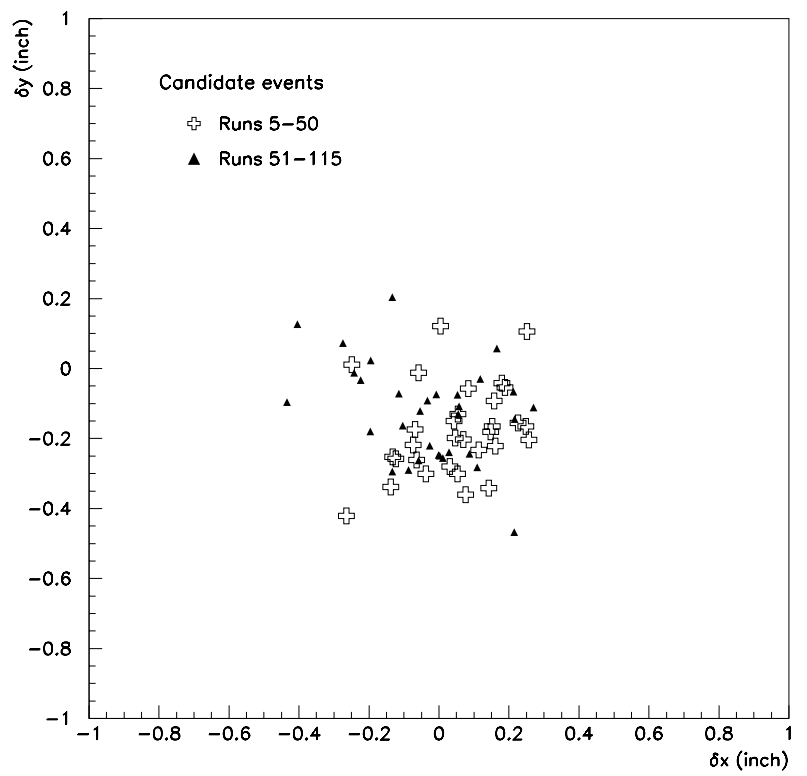

Figure 6.28: Effective size of the antihydrogen beam. Position spread of candidate events at the foil location corrected for beam position. 


\subsubsection{Emittance Measurements}

The emittance of the beam can be ascertained by looking at the tracks recorded by E862. Figures $6.29,6.30,6.31$, and 6.32 show the transverse phase space plots $\left(x, \theta_{x}\right),\left(y, \theta_{y}\right)$ for triple coincidence events and all tracks. The emittance is the area of the occupied phase space, $\epsilon_{x}=\pi \cdot \sigma_{x} \cdot \sigma_{\theta_{x}}$. An ellipse has been drawn to give an idea of how much phase space area the points fill up and what emittance this corresponds to. However, the ellipse shown is not a fit and does not correspond exactly to an ellipse which encloses $95 \%$ of the particles. Furthermore, the ellipse is centered at the origin. The events represented in the first plot, figure 6.29, form an occupied area which is clearly not centered at the origin. In addition, the position and angle offsets of the beam have not been removed. 


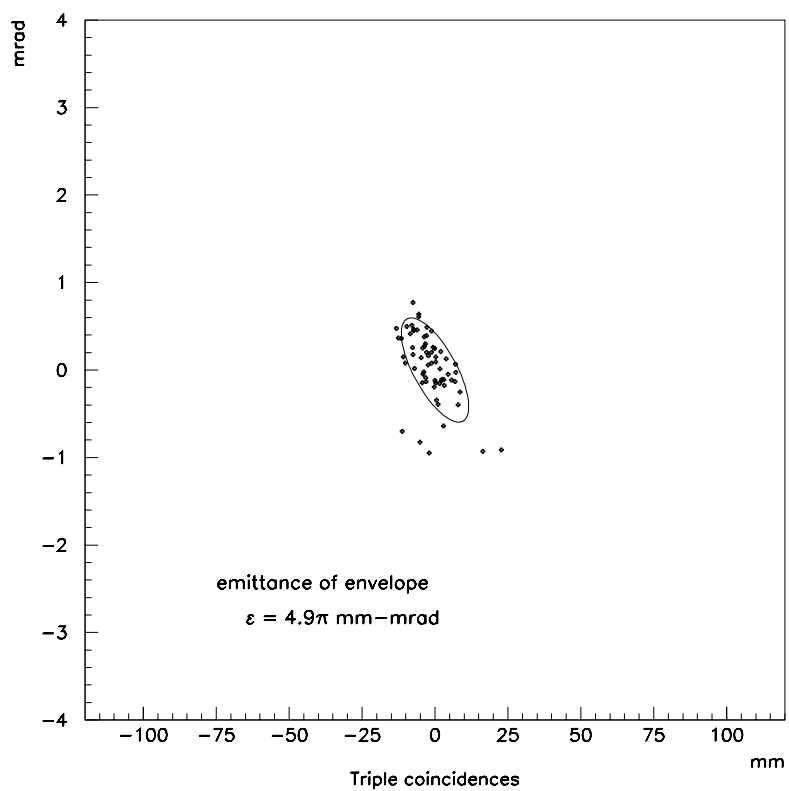

Figure 6.29: X-Phase space plot of tracks for candidates.

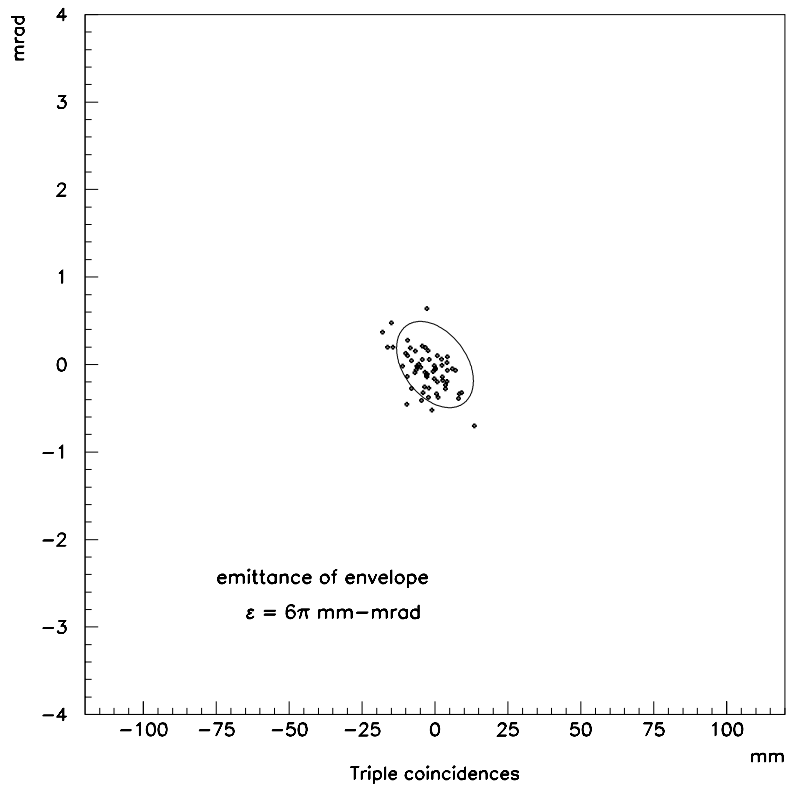

Figure 6.30: Y-Phase space plot of tracks for candidates. 


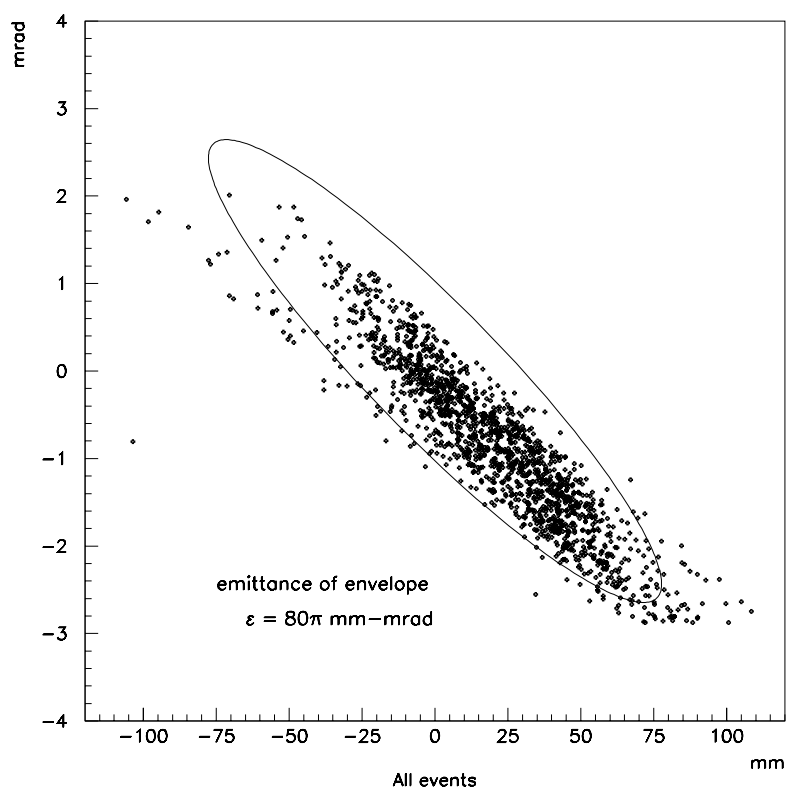

Figure 6.31: X-Phase space plot of tracks for all tracks.

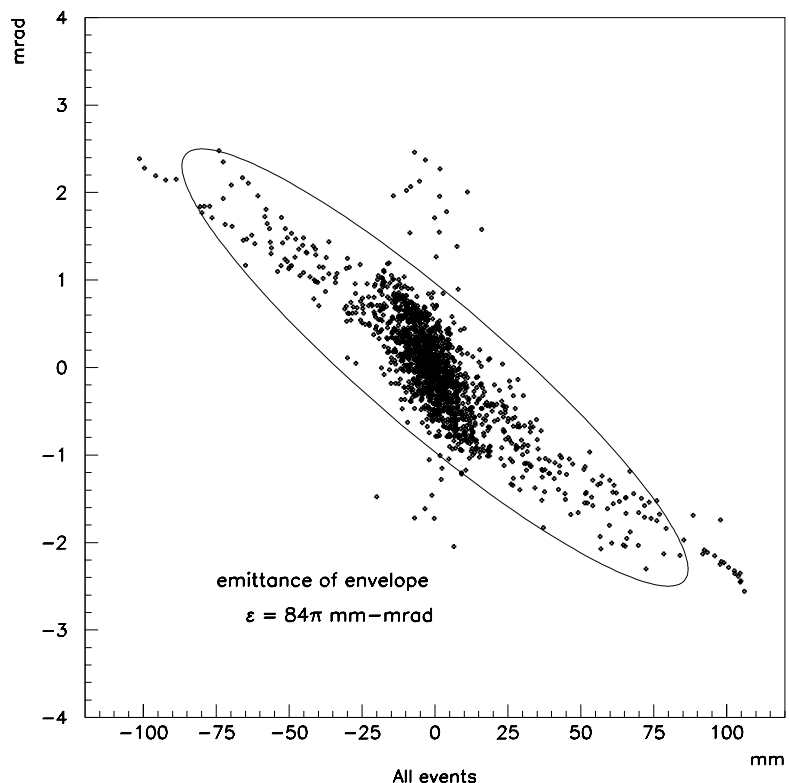

Figure 6.32: Y-Phase space plot of tracks for all tracks. 


\subsubsection{Profiles at jet}

The track is extrapolated back to the gas jet by projecting where the PWC \#2 space point would be if there were no bend and then drawing a straight line through the space point in PWC \#1, (see figures 6.35, and 6.36). The track positions at the location of the jet in figure 6.36 form a rectangular profile, which may correspond to the rectangular shapes of the dipole magnets' vacuum pipes. This could be due to either the A5B3 dipole whose vertical aperture is only $1.45 \%$ (ID) or one of the antiproton bend magnets P1, P2 which have apertures of 2.0" (ID). Figures 6.37, and 6.38 show the distributions for events separated into above and below transition sets. Alongside the triple coincidence positions are positions for high momentum events without evidence of a positron. Those below transition seem to fill a large region consistent with a rectangular beampipe profile. The $\mathrm{e}^{+}$dipole magnet adds a small but noticeable offset of 0.4 ". The candidate track positions at the location of the jet are offset from the origin (see figure 6.35).

One may ask if there is a difference in angular distributions of tracks from triple coincidence events and tracks from high momentum events with no $e^{+}$by looking at their angular distributions. If the latter are indeed mostly scatters off a vacuum pipe, then the angular distribution should at least fill the angular acceptance of the antiproton spectrometer. Figures 6.33, and 6.34 compare these distributions. Although the triple coincidence events' angular distribution is thinner and clearly more gaussian in shape, the high momentum (no $e^{+}$) angular distributions are not flat. The $\mathrm{X}$ angles have been corrected by -0.38 inch due to the $e^{+}$dipole bend. 

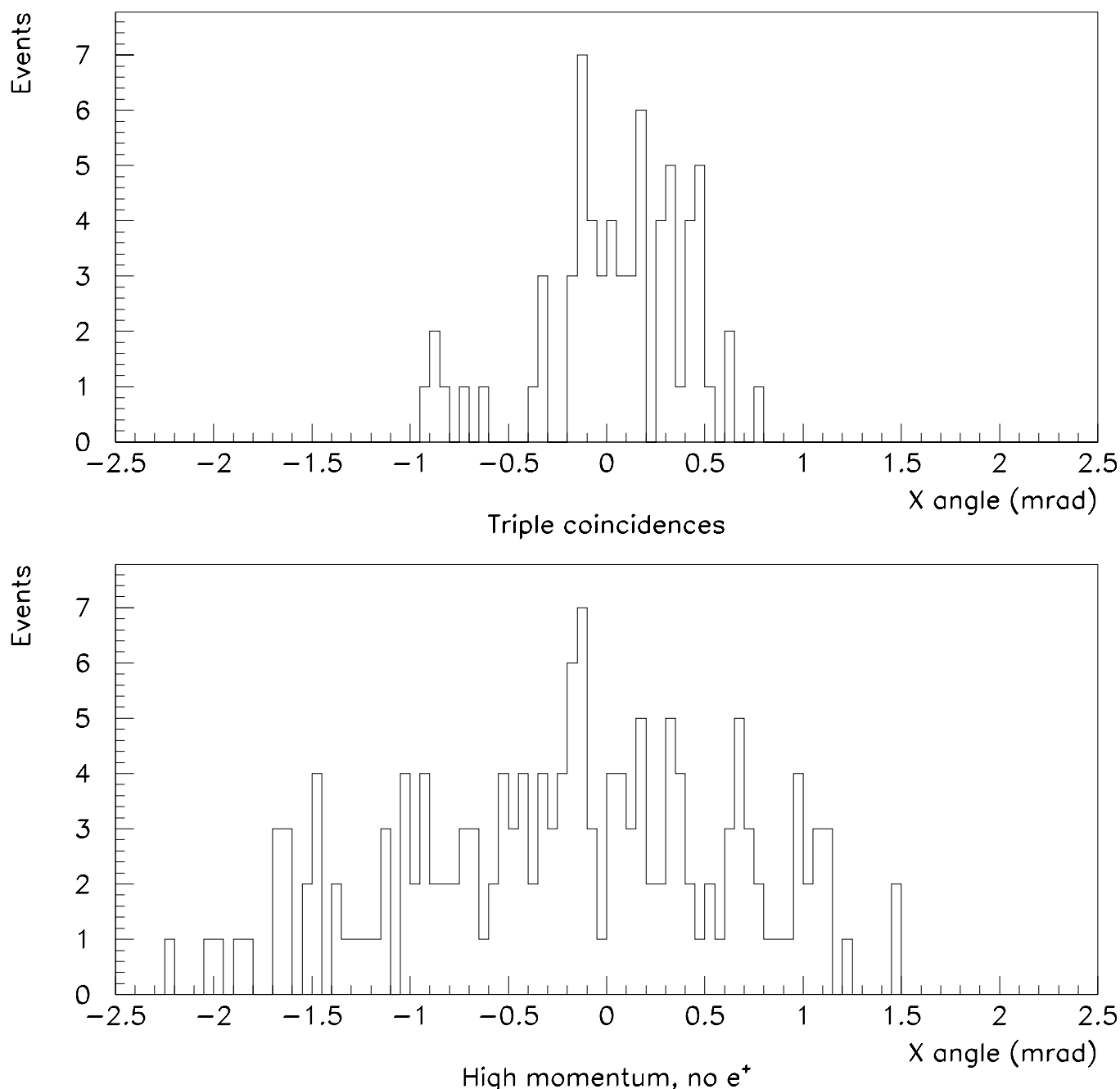

Figure 6.33: $\mathrm{X}$ angular distributions for triple coincidences and high momentum tracks without a $e^{+}$. An offset of 0.4 inch from the small dipole has been removed. 

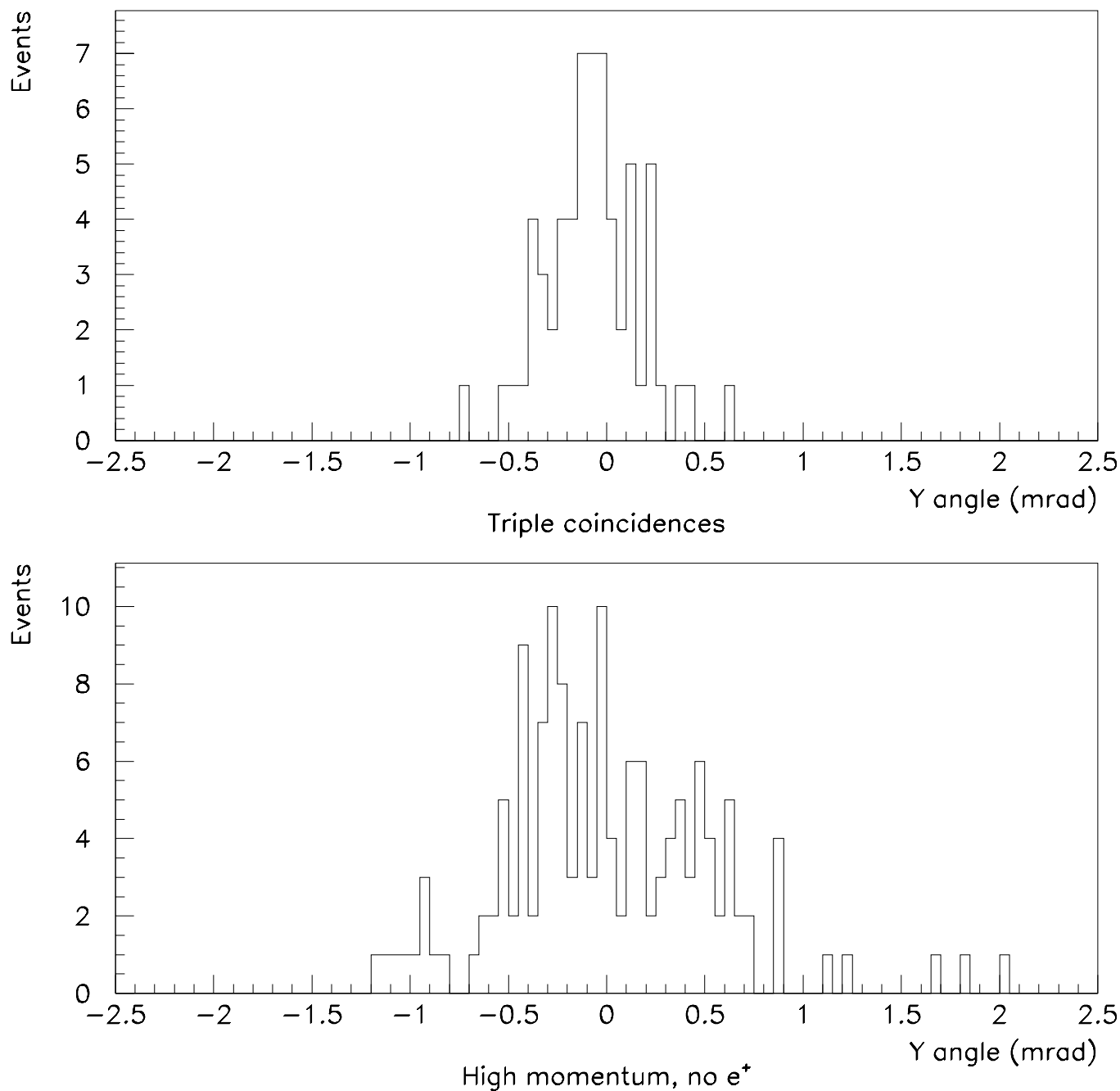

Figure 6.34: $\mathrm{Y}$ angular distributions for triple coincidences and high momentum tracks without a $e^{+}$. 


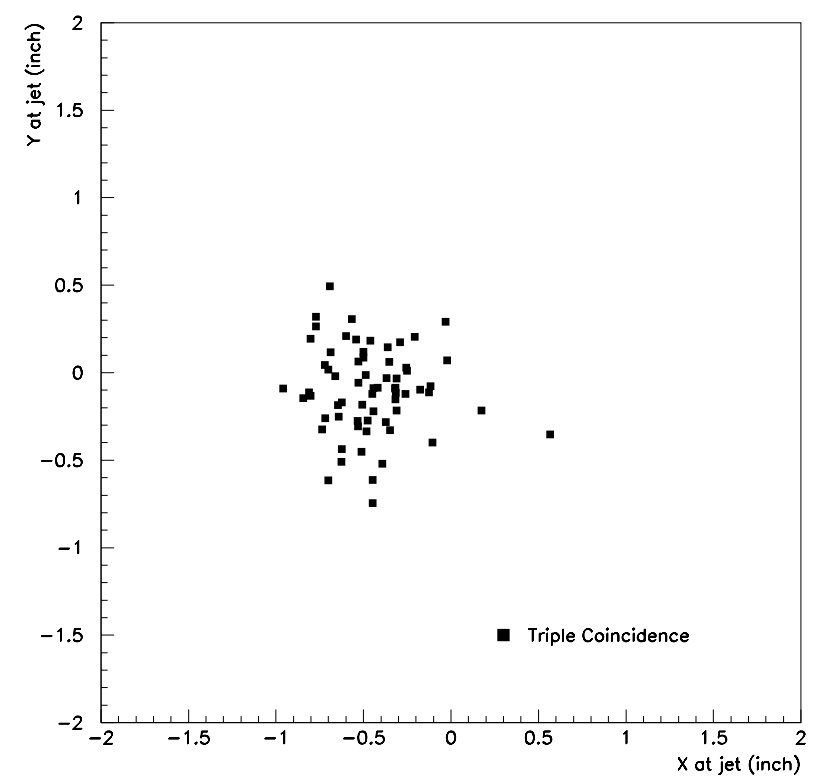

Figure 6.35: Extrapolated position of track at gas jet for triple coincidence events. The track positions are offset horizontally from zero because of the $e^{+}$dipole (by $\left.0.4^{\prime \prime}\right)$.

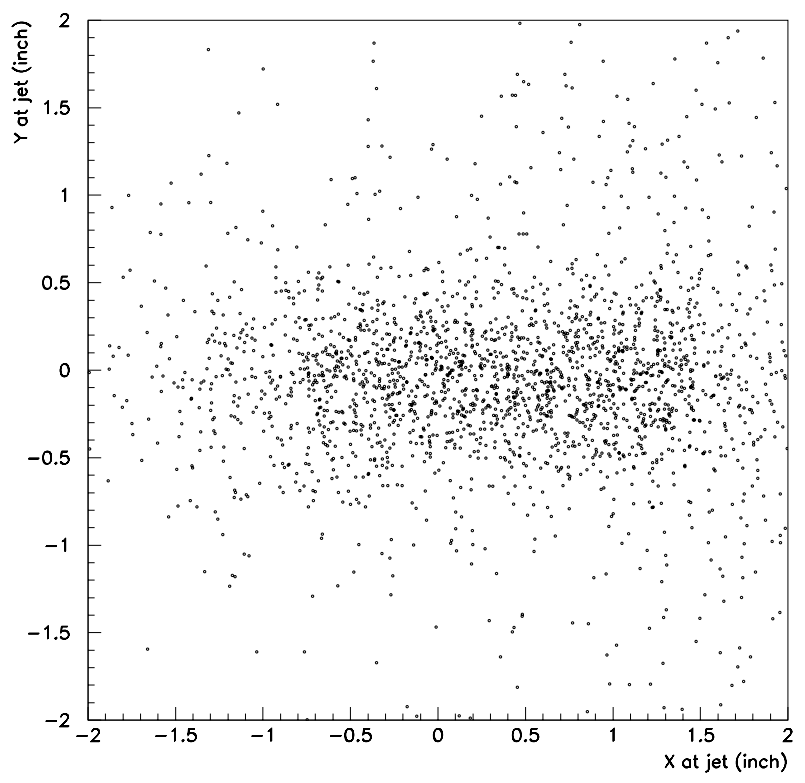

Figure 6.36: Extrapolated position of track at gas jet for all good tracks. The track positions are offset horizontally from zero because of the $e^{+}$dipole (by $0.4 "$ ). 


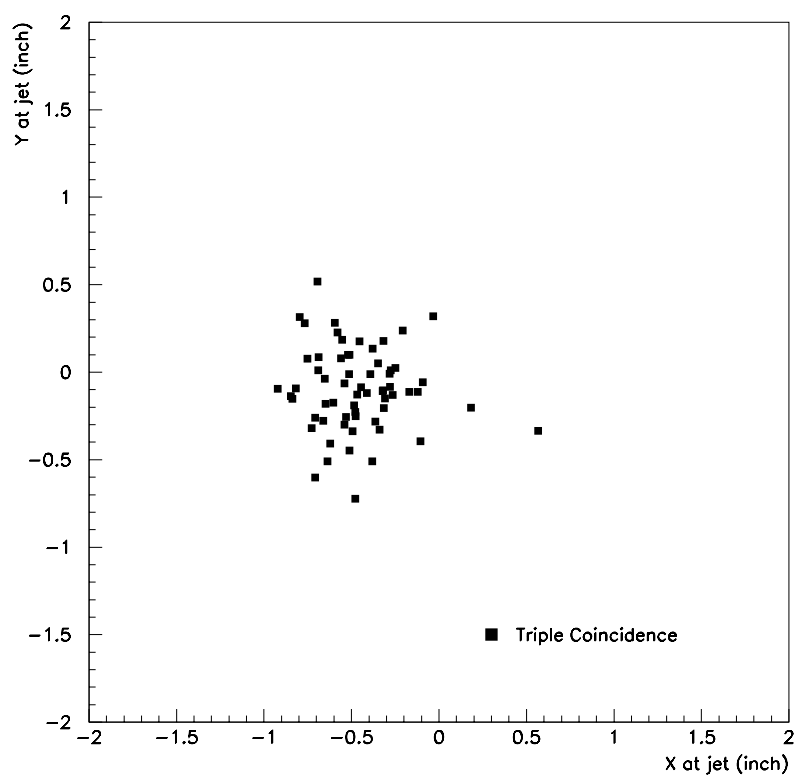

Figure 6.37: Extrapolated position of track at gas jet for triple coincidence events, above transition. The track positions are offset horizontally from zero because of the $e^{+}$dipole (by $0.4^{\prime \prime}$ ).

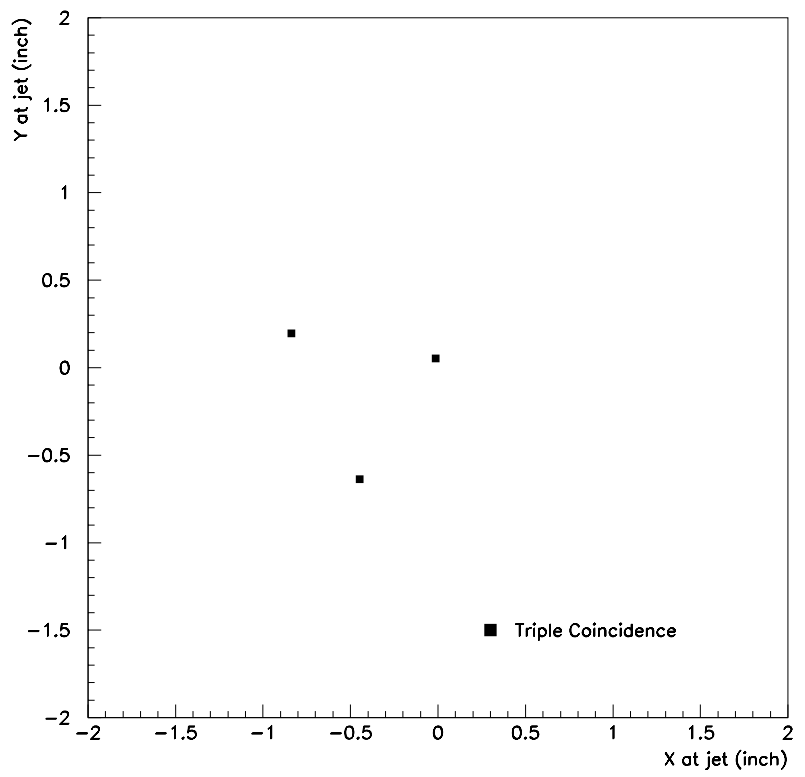

Figure 6.38: Extrapolated position of track at gas jet for triple coincidence events, below transition. The track positions are offset horizontally from zero because of the $e^{+}$dipole (by $0.4 "$ ). 


\subsubsection{Positron Energies and Annihilation Spectra}

Of the 76 triple coincidence events, none had the veto counters on. This indicates that the particle hitting the $e^{+}$counter came from the direction of the $e^{+}$ spectrometer. The $e^{+}$counter energy deposits for all but one of the 76 clustered around the energy value expected from the calibration data. None had energy deposits much larger than expected. A signal corresponding to a MIP is expected to leave a much larger energy deposit in the counter.

There is a difference between data before and after January 1, 1997, since the TF1 counter's threshold was lowered, (see section 6.6.7). This caused the triggers' start signal as well as the ADC gates to be earlier and the ADC values larger. Previously, the ADC signals were cut off on the front end by a small amount and had lower integrated values as a result. There was expected to be a change of the gain when the 1 " diameter scintillator (BC-418) was replaced with a 2 " diameter scintillator (BC-420) but none was observed (April 1997). In addition, the responses for electrons and positrons were compared. No difference resulted.

The dependence of ADC values on dipole current was fit for the two cases and used to convert to $\mathrm{e}^{+}$energy (see figure 3.30). The distribution of $e^{+}$pulse heights, (in ADC counts out of a full scale of 1024), is shown in figures 6.39, and 6.40, with the 1996 and 1997 fits to the calibration data, respectively. The pulse heights for the triple coincidence events agree with the extrapolations to the calibration data. Figures 6.41 , and 6.42 show the deviation of the triple coincidence candidate events from the extrapolation for 1996 and 1997 data sets, respectively. During April 1997, the $1 "$ counter was replaced by the $2 "$ counter but no change in gain was observed after repeated testing even though the scintillator material is different as well. Thus only two sets of gain constants are used. 


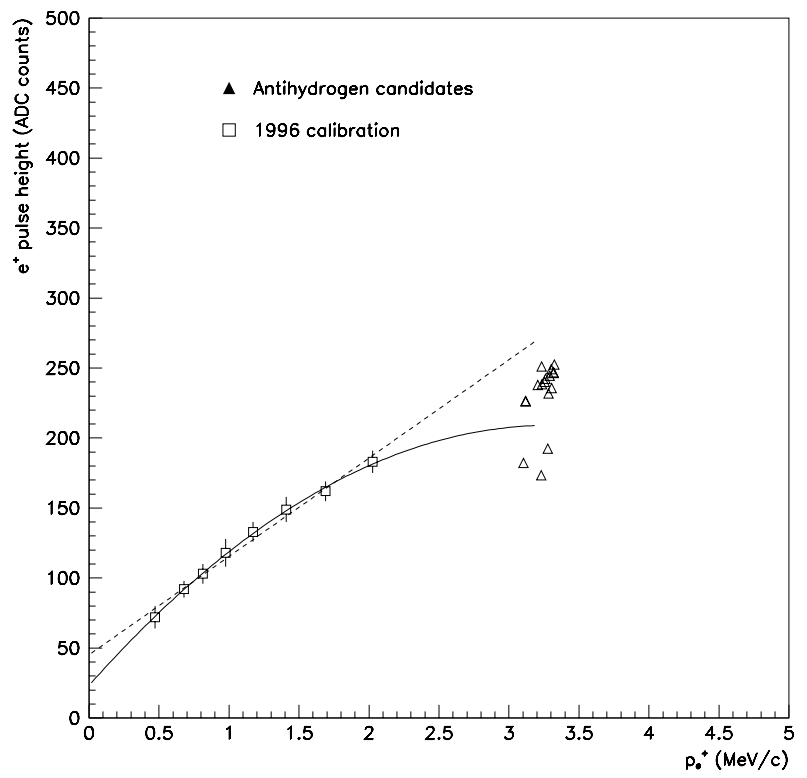

Figure 6.39: Pulse height of $\mathrm{e}^{+}$scintillator vs. $e^{+}$momentum for triple coincidences during 1996. A linear and a quadratic fit to the calibration data are shown.

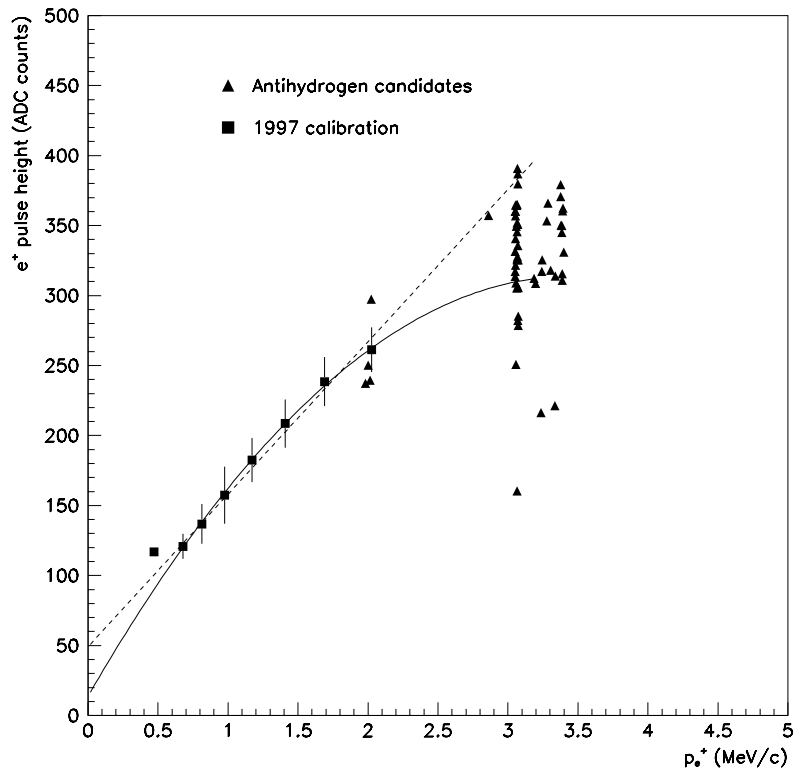

Figure 6.40: ADC of $\mathrm{e}^{+}$scintillator vs. $e^{+}$momentum for triple coincidences during 1997. A linear and a quadratic fit to the calibration data are shown. 


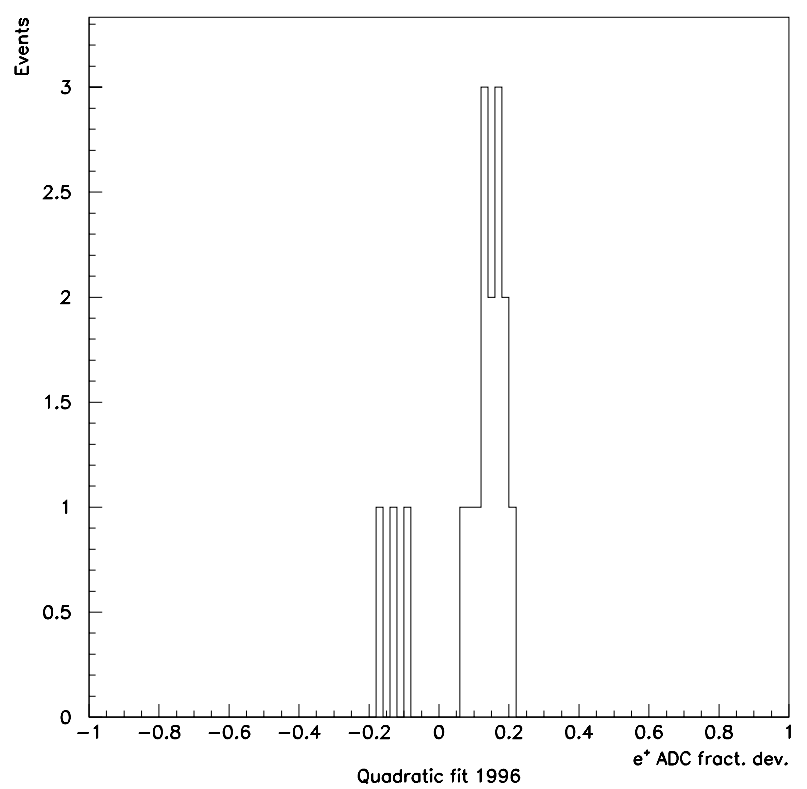

Figure 6.41: Deviation of e+ pulse heights from quadratic fit for 1996 triple coincidences.

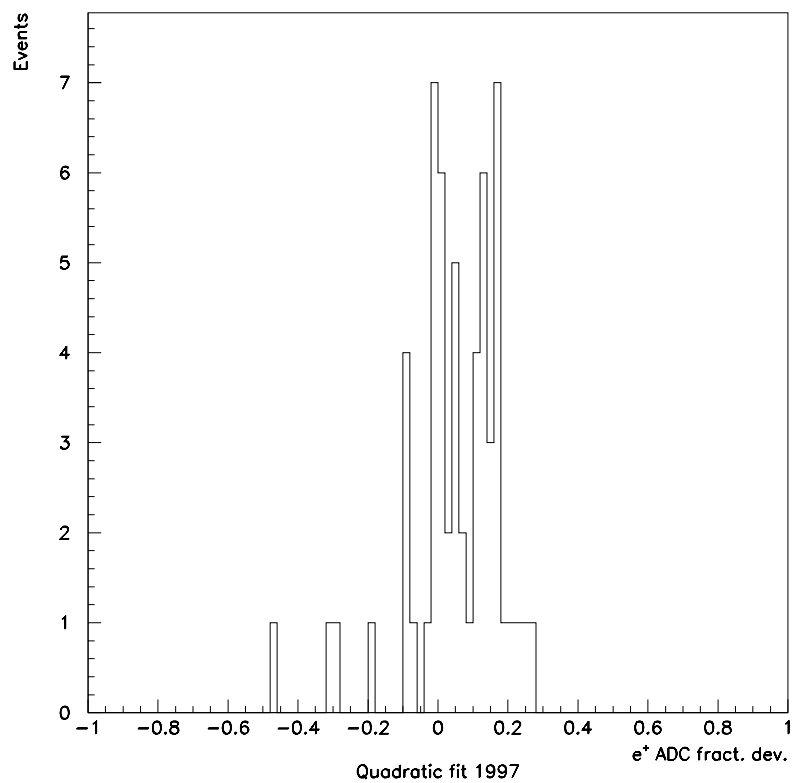

Figure 6.42: Deviation of e+ pulse heights from quadratic fit for 1997 triple coincidences. 
Figure 6.43 shows the e + pulse height distributions for triple coincidence events superimposed on top of the same distribution for all events, except the number of events per bin has been scaled down by a factor of $1 / 1000$. The ADC values of the events from 1996 are scaled by a factor representing their different gain constant. The constant used here is the $e^{+}$peak of the pulse height distribution taken at the peak of the $\beta^{+}$spectrum during calibration. Here the separation of the pulse heights from the majority of the smooth background distribution is emphasized. If the triple coincidence pulse height distribution were much more spread out, one might argue that the hits were from other particles in the environment. They are all contained in one small area. In addition, the dependence on beam momentum has not been removed. Although the pulse height has been observed not to be exactly proportional to kinetic energy deposited, there is still a well-behaved dependence on the $e^{+}$momentum. The momentum dependence has not been removed in the previous figure but it has been removed in figure 6.44. Figure 6.45 shows the distribution of $e^{+}$pulse heights for events with one track only. Those events with one track that have a pulse height are above pedestal, (about 60 counts), are almost all antihydrogens. Events with a lot of spray from the jet region almost always have more than one track, so these are excluded. 


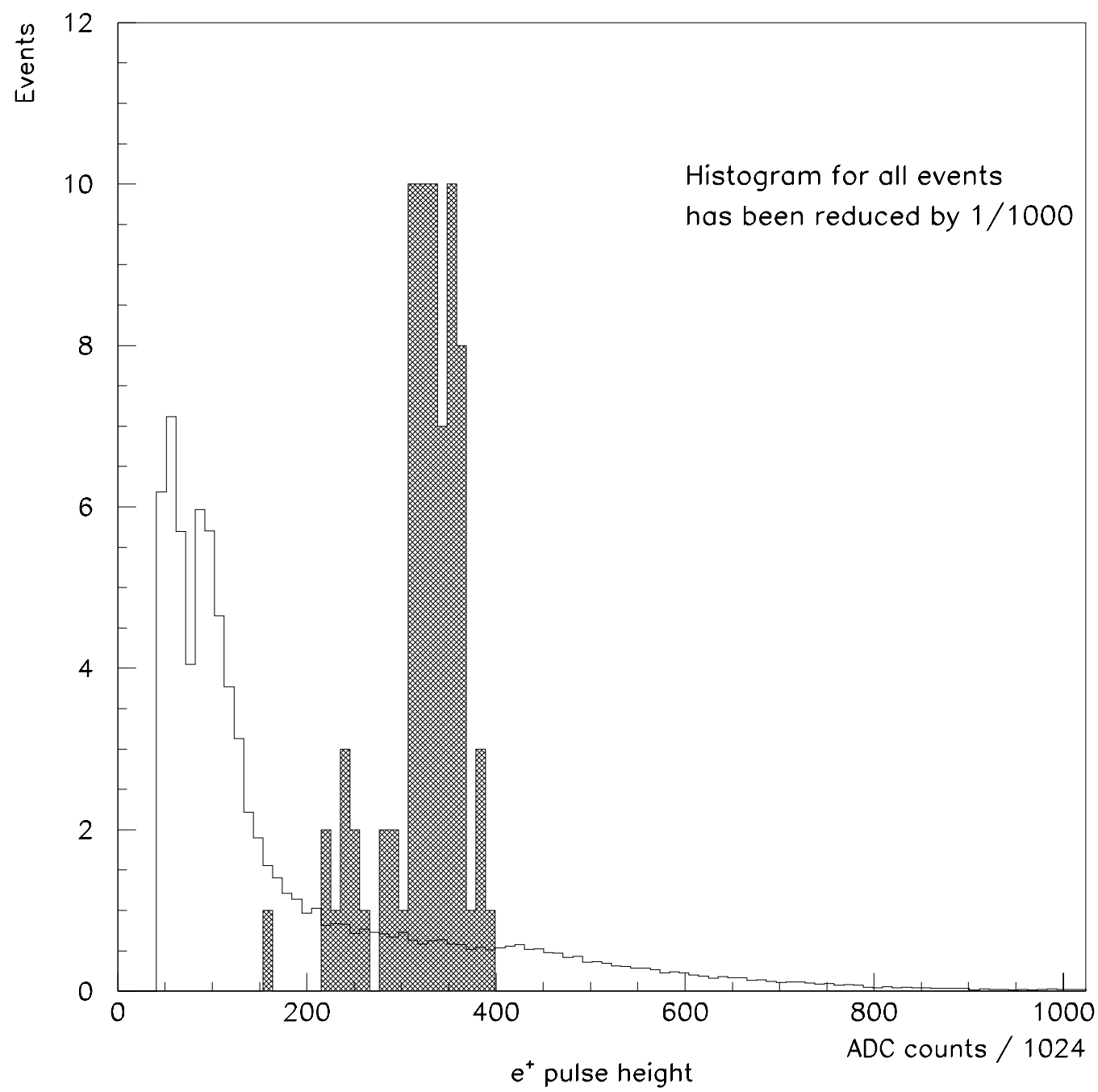

Figure 6.43: $e^{+}$pulse heights for triple coincidence events superimposed on the distribution of pulse heights for all events. Events before run 20 have been corrected for a different gain constant. The histogram representing all events has been scaled down by a factor of $1 / 1000$. 


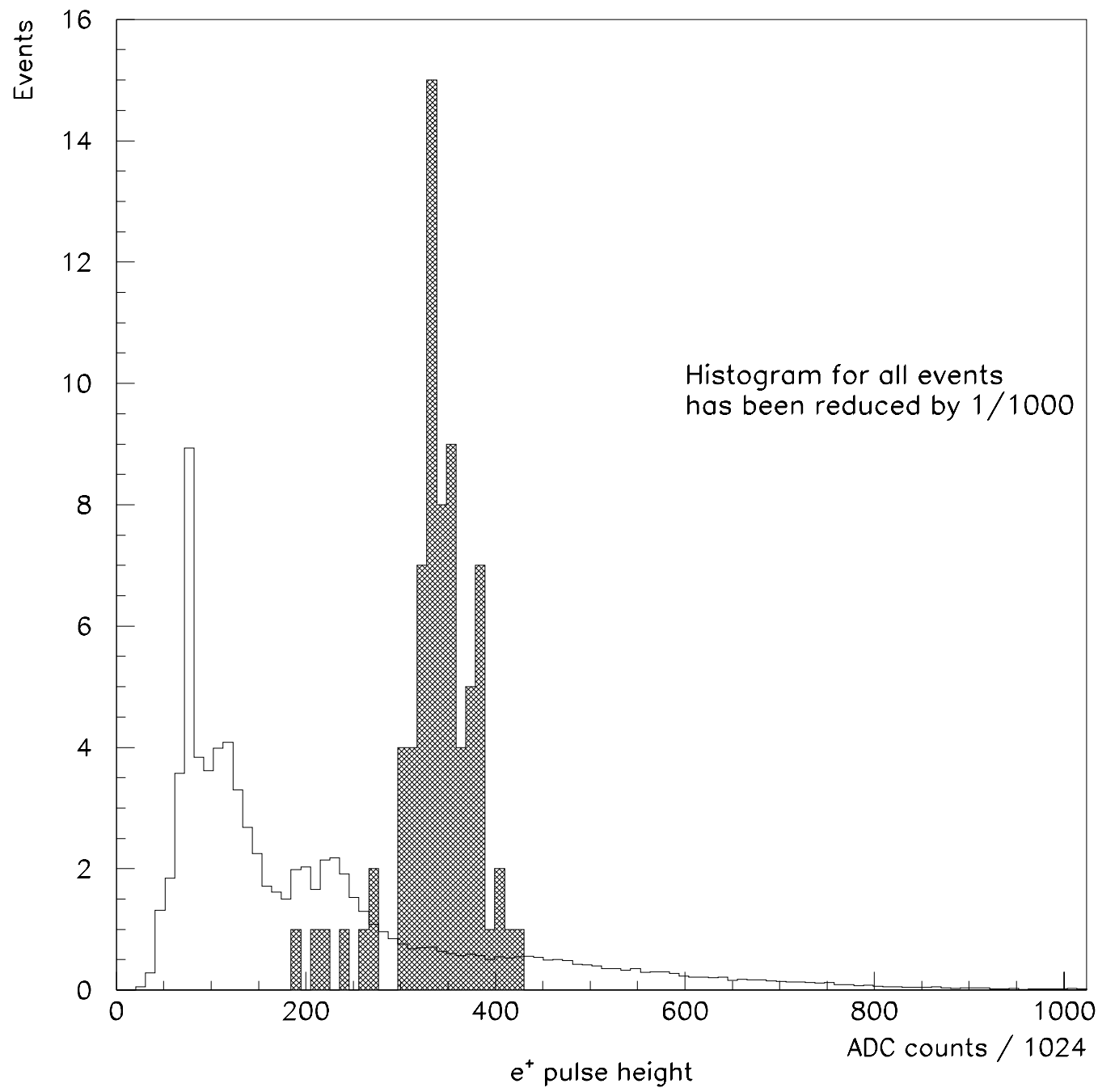

Figure 6.44: $e^{+}$pulse heights for triple coincidence events superimposed on the distribution of pulse heights for all events. Events before run 20 have been corrected for a different gain constant. The histogram representing all events has been scaled down by a factor of $1 / 1000$. Beam momentum dependence has been removed. 

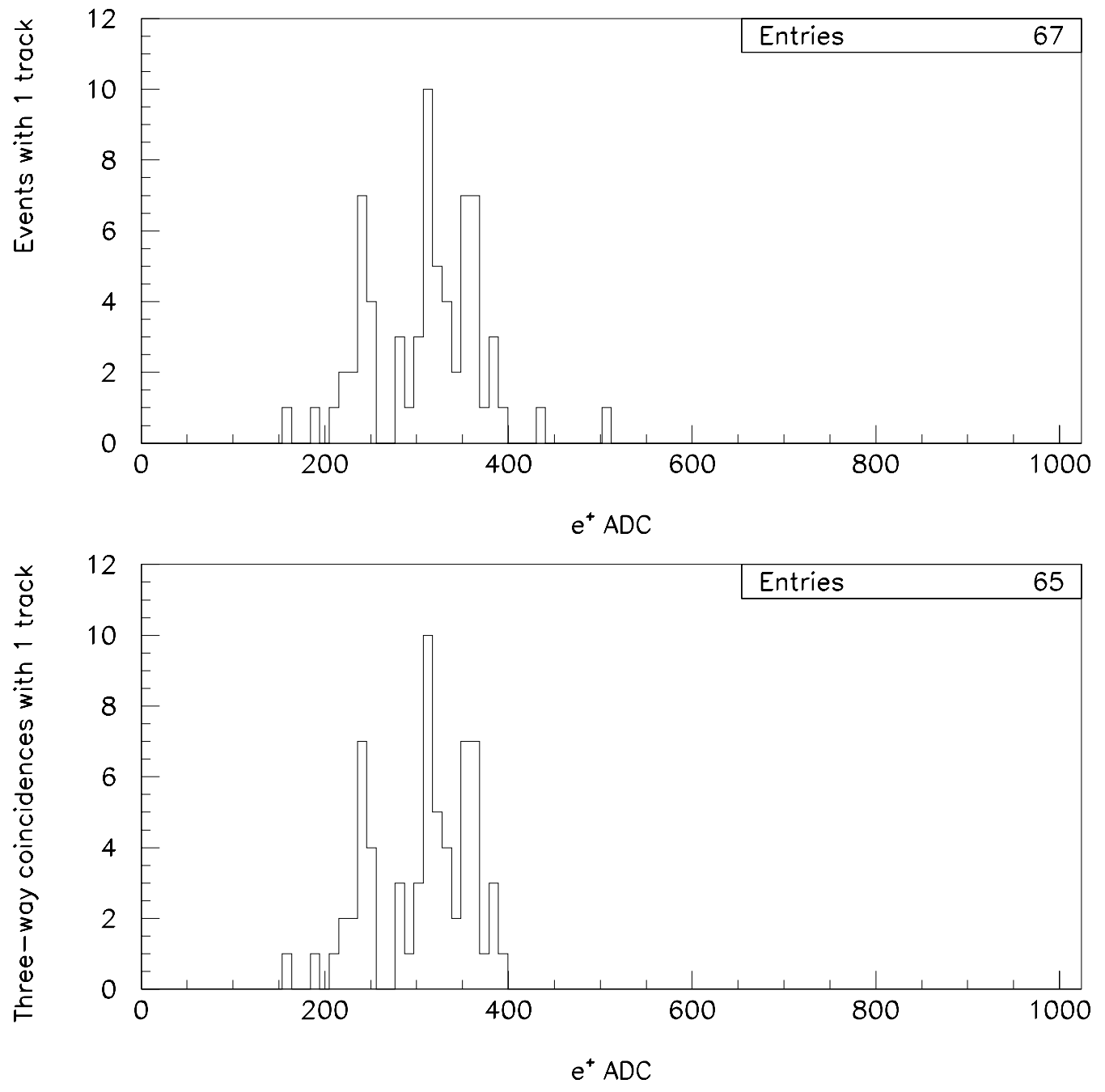

Figure 6.45: $e^{+}$pulse heights for all events with one track and three-way coincidences with one track. 
The NaI energy deposits are trickier since the detector is much less efficient than the $e^{+}$counter. The distribution of $\mathrm{NaI}$ energy for candidates is the same as what is seen in the calibration data though (see figure 6.46) taken with the germanium $\beta^{+}$ source. Figures 6.48 , and 6.47 show the correlation of energies in the $\mathrm{NaI}$ halves for triple coincidence candidate events and all events respectively. There are no huge energy deposits in the candidate data which would indicate high energy photons (at least a few $\mathrm{MeV}$ ). The sodium iodide energy deposits are not used as a strict cut since the efficiency of seeing two $511 \mathrm{keV}$ photons is only $30 \%$, (see table 3.5 ). Clearly, many events have both energies corresponding to $511 \mathrm{keV}$ annihilation gamma rays. This still represents only about $30 \%$ of the total. Approximately, another $30 \%$ have energies in the bands where one energy is about $511 \mathrm{keV}$ and another has lost some energy due to a Compton scatter. Furthermore, some events exist along the band where the sum of energies adds up to $2^{*} 511 \mathrm{keV}$. These indicate the scattered photon was not lost but instead, entered the other half to contribute its energy there. Finally, a very small number of events exist with larger energies in both halves. The events where one of the energies is still in the $511 \mathrm{keV}$ band are probably due to the other energy value resulting from a photon which has a conversion very close to one of the three phototubes. The increased solid angle may yield a larger pulse height than average. The others are, most likely, annihilations in flight. The calibration data shows this effect too and the proportion of events in the calibration data and the candidates is approximately the same. Those events for which

$$
E_{\gamma, 1}+E_{\gamma, 2} \leq 2 * 511 k e V
$$

is satisfied were not cut. The reasons for an asymmetric energy deposit in the NaI are discussed in section 3.6.3.

Very few background positrons were seen, probably because any created in or near the jet would be bent by magnetic fields far upstream. Neutral pion conversions 


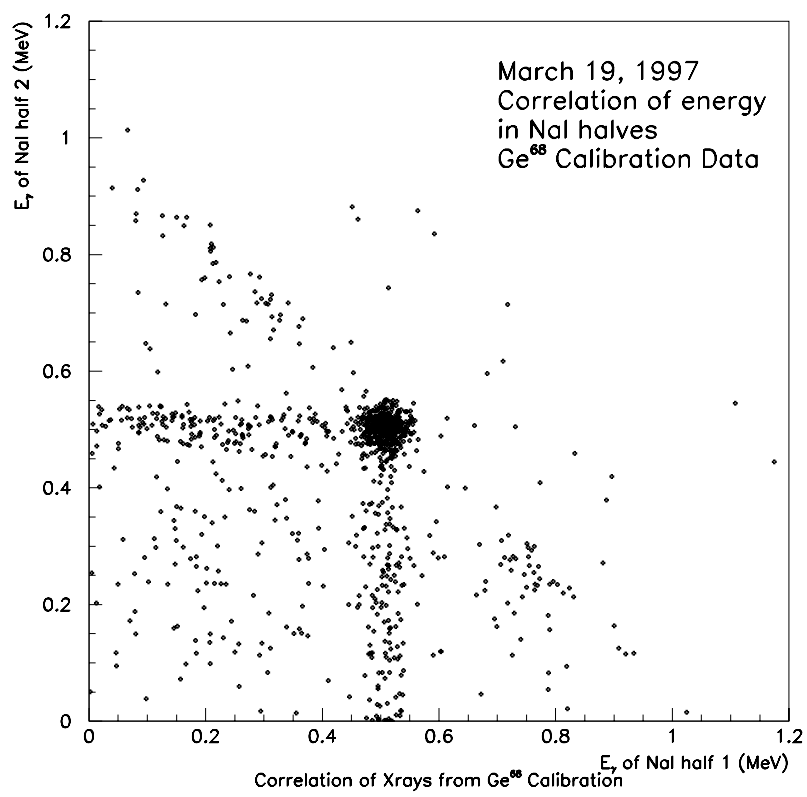

Figure 6.46: Correlation of $\mathrm{e}^{+}$annihilation ray energies in sodium iodide for calibration data

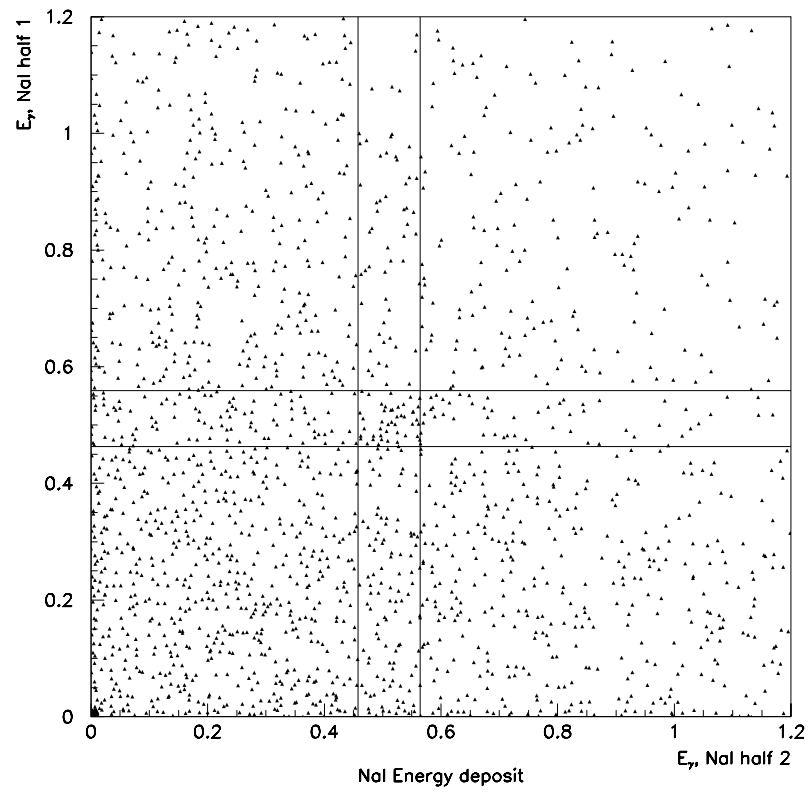

Figure 6.47: Correlation of annihilation $\mathrm{X}$ ray energies in $\mathrm{NaI}$ detector for all events in data. 


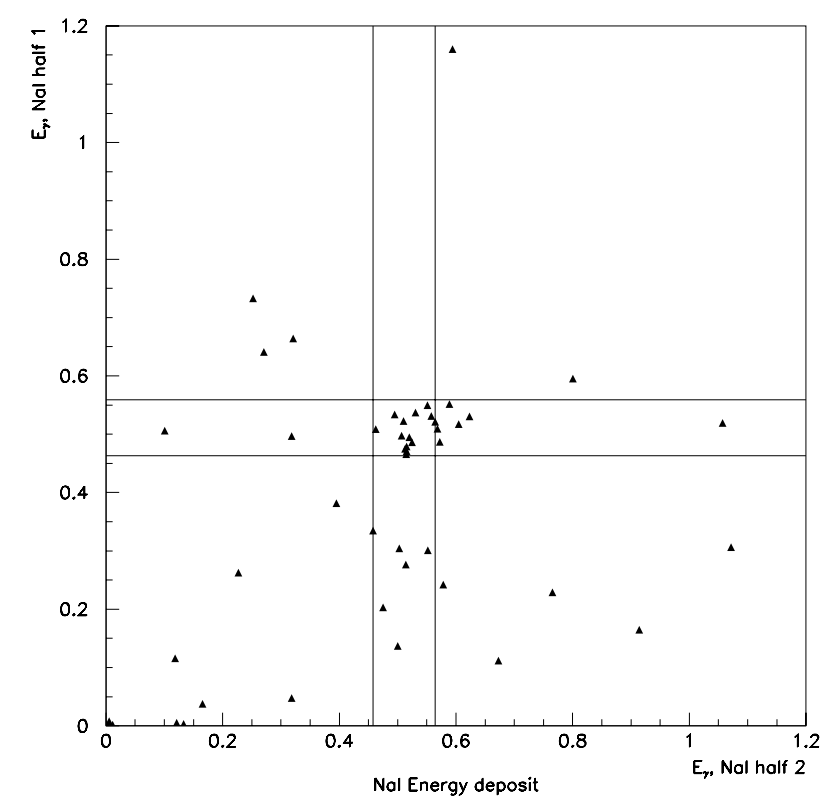

Figure 6.48: Correlation of annihilation $\mathrm{X}$ ray energies in $\mathrm{NaI}$ detector for candidate events in data.

with a Dalitz decay are frequent but they need to have the correct trajectory. For example, for two pion modes, $d \sigma_{\pi^{\circ} \pi^{\circ}} / d \cos \theta^{*}$ is probably $\sim 1 \mu b$ near $\theta=0$ at $6 \mathrm{GeV} / \mathrm{c}$. The $\pi^{o}$ decays almost always to $\gamma \gamma$. One of the photons needs to travel with a trajectory close enough to an antihydrogen that hits the foil. The probability that the photon produces an $e^{+} e^{-}$pair near the foil determines the number of events the experiment should have seen in $120 \mathrm{nb}^{-1}$ to be about $2 \times 10^{-2}$. Further reductions come from the positron needing to have the correct trajectory and energy to traverse the $e^{+}$spectrometer. 

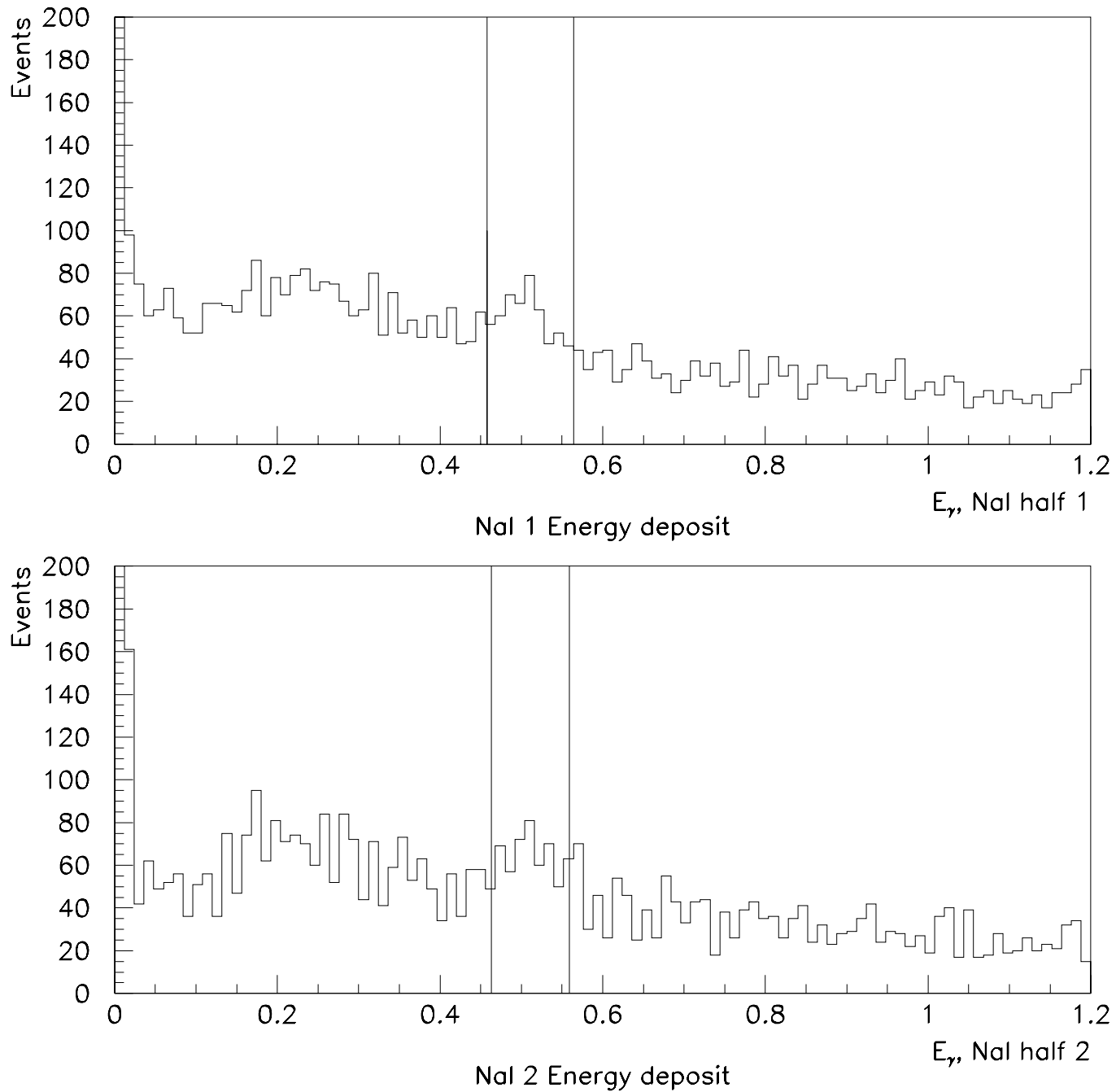

Figure 6.49: Annihilation $\mathrm{X}$ ray energy distributions from the NaI detector for all events in foil-in data. 

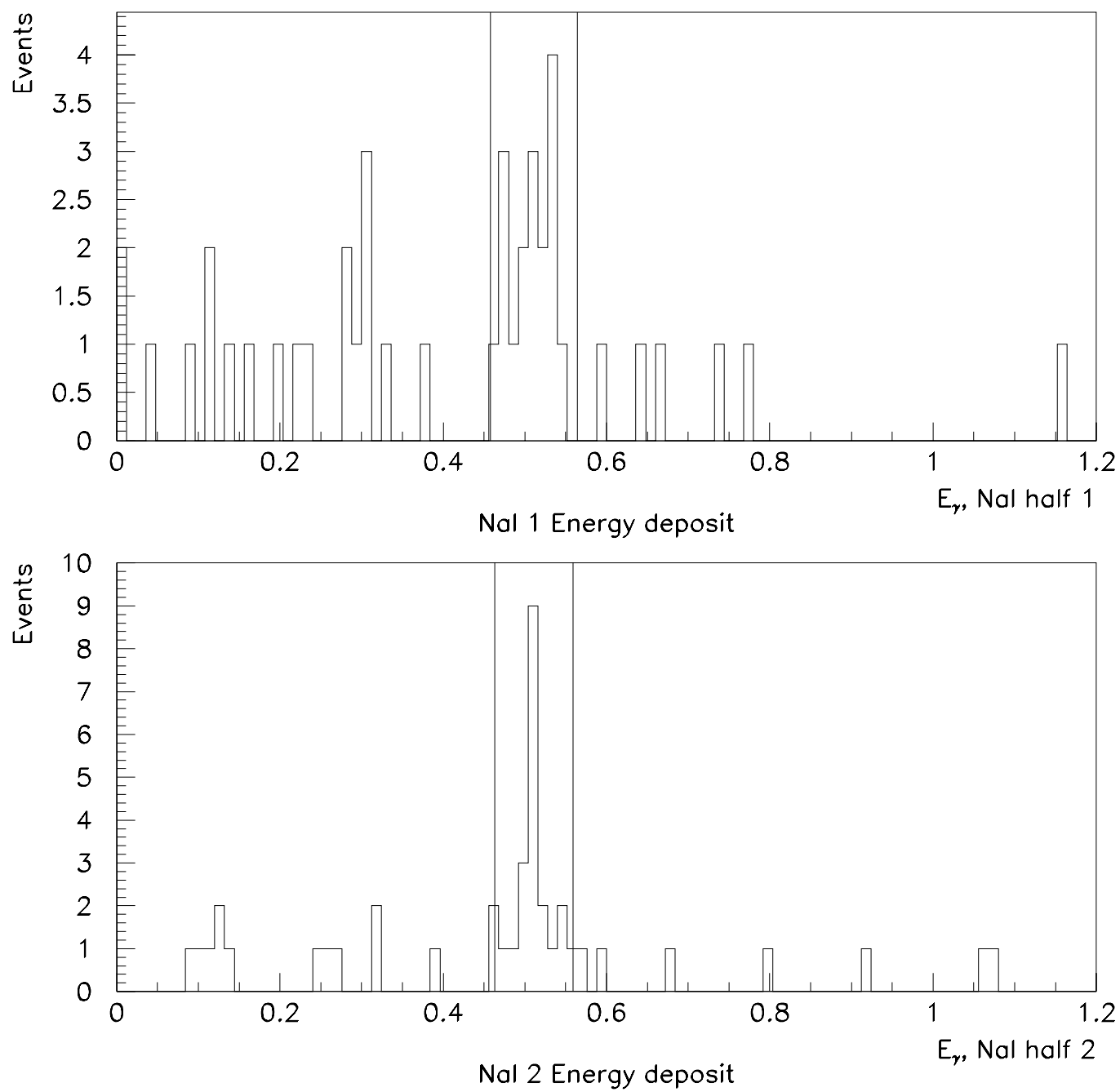

Figure 6.50: Annihilation $\mathrm{X}$ ray energy distributions from the NaI detector for triple coincidence events in foil-in data. 


\subsubsection{Timing Analysis}

The antiproton time of flight distributions are found by the difference in arrival times between the TF1 and TF2 counters after corrections are applied. The correlations of the time of arrival with the pulse height in the ADC and track positions are taken into account by using a correction determined from accumulating and fitting all good tracks which triggered both counters. The timing distributions of the TF1 and TF2 counters were correlated to the particle's position when passing through the scintillator counter, (see figure 6.51). Since each counter was close to the corresponding wire chamber in the longitudinal direction, the wire chamber hit position was used as the particle's position in the counter.

The corrections to the arrival times for the time of flight counters are calculated by two methods. The first averages the signals from the two PMTs on each counter. The advantage to this is that no fitting error results. It can be used to remove the position dependence of a hit but not an arrival larger pulse height in both PMTs. If both PMTs have the same gain, they will see equal pulse heights and arrival times for a particle passing equidistant between them. A hit that is nearer to one of the phototubes will generate more light near that phototube, less in the other and will be averaged out. The problem is that two particles that leave substantially different $d E / d x$ have different arrival times because of slewing. If the two time of flight counters had the same thickness (which they don't), this would cancel in principle.

The second method corrects the timing distributions of each counter for pulse height and position dependence independently. As described in section 6.4 , the background tracks are predominantly antiprotons very close to beam momentum. Their pulse height spectrum and corresponding arrival times are expected to be distributions with small widths. Once the particle's position dependence has been removed, 
the arrival times are not correlated with the pulse height spectra. It will suffice then to use the averaging method for calculating the arrival time and the time of flight.

For the following analysis, a sample of events was defined by demanding that the number of tracks be equal to one (except for candidate events), the absolute $Y$ residual of the primary track be less than 0.15 inch $\left(\left|Y_{\text {res }}\right|<0.15\right)$ and that the relevant TDC channels all have in-time hits. They are all the above transition momentum of the antiproton source. In addition, for the time of flight distributions, the ratio of reconstructed momentum to beam momentum must be within the range, $(0.9975<$ $\left.\frac{p}{p_{\text {beam }}}<1.0015\right)$. The fractional momentum and $\mathrm{Y}$ residual are discussed in more detail in section 6.3. A linear correction was applied to TDCTF11 vs XSP1, the horizontal space point in the first wire chamber. This also removed the correlation of TDCTF11 with ADCTF11 and ADCTF12, (see figures 6.52, and 6.53). A correlation with TDCTF12 had to be further removed. The TDCTF21 and TDCTF22 distributions have a dependence on the YSP3 (the vertical space point in the last chamber), (see figure 6.54), since these PMTs are aligned on top and bottom of the 4 " wide counter. The dependence of one PMT's arrival times on YSP3 is the reverse of the other PMT's arrival times.

$$
\begin{aligned}
& T D C T F 21 \sim+.25 * Y S P 3 \\
& T D C T F 22 \sim-.25 * Y S P 3
\end{aligned}
$$

Any further dependence of TDCTF21 on ADCTF21 or ADCTF22 is not evident as seen in figure 6.55. The TF12 signal was the timing start (or strobe) for the two higher rate triggers. The difference TDCTF11 - TDCTF12 is used to establish the timing resolution of the TF1 counter as shown in figure 6.56 with a resulting $\sigma=0.4$ ns spread after the correction for position is made. The resolution for TF2 is shown in figure 6.57 with a resulting $\sigma=1.3 \mathrm{~ns}$ spread. Data with different beam momenta (and thus different $\beta$ ) have been used so this has been factored out and the time of 


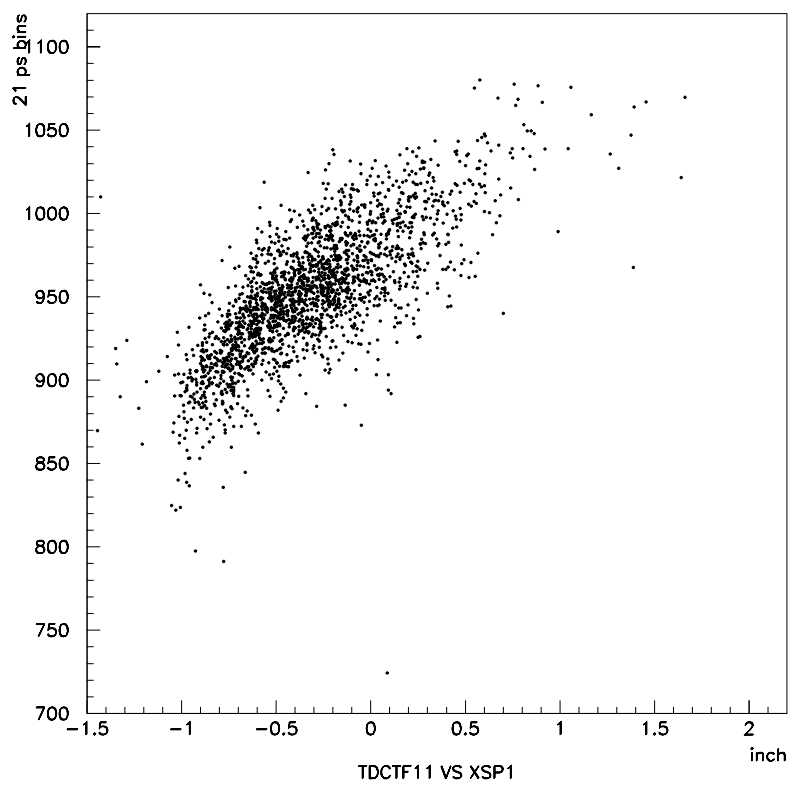

Figure 6.51: Correlation of TF11 arrival times with horizontal position.

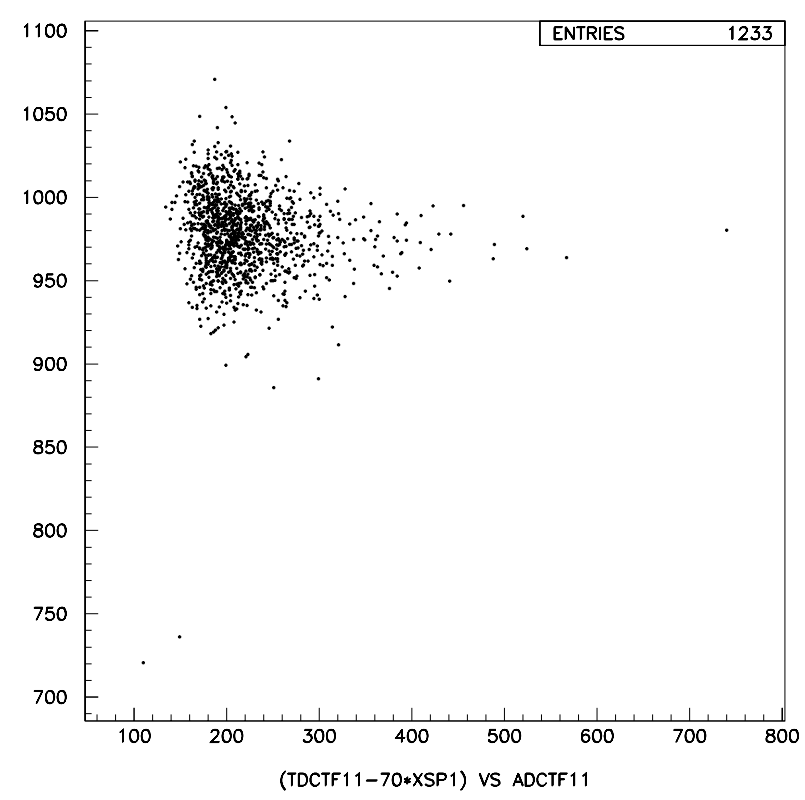

Figure 6.52: Correlation of TF11 arrival times with ADC TF11 after removing position dependence. 


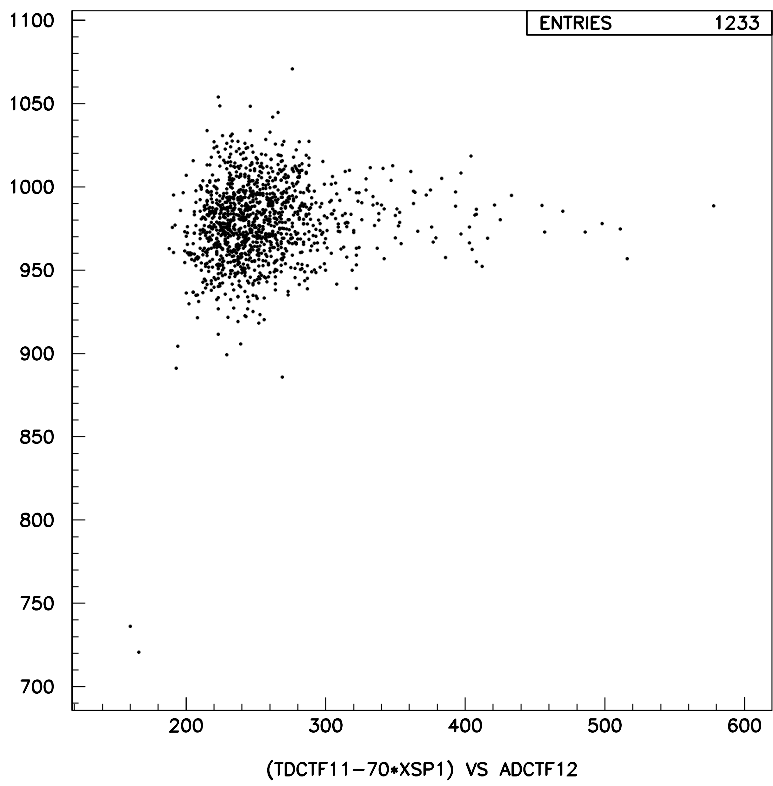

Figure 6.53: Correlation of TF11 arrival times with ADC TF12 (trigger start PMT) after removing position dependence.

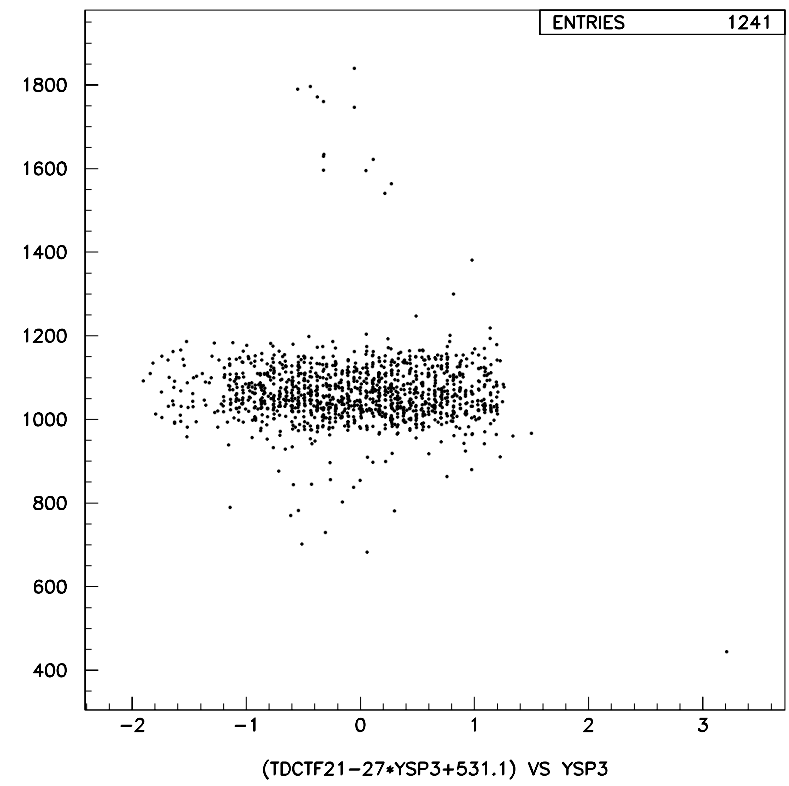

Figure 6.54: Correlation of TF21 arrival times with vertical position. 


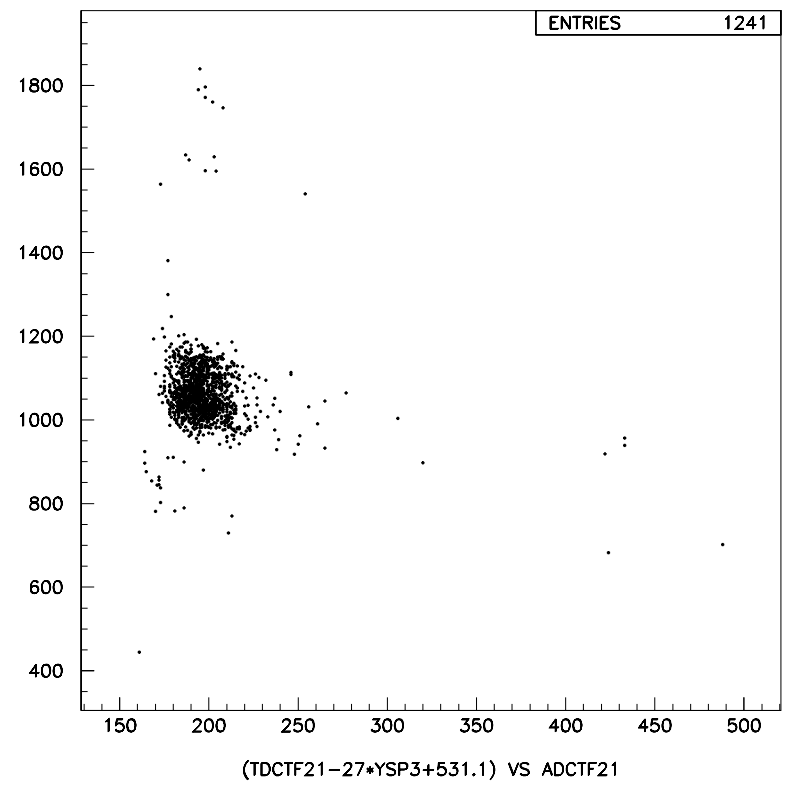

Figure 6.55: Correlation of TF21 arrival times with ADC TF21.
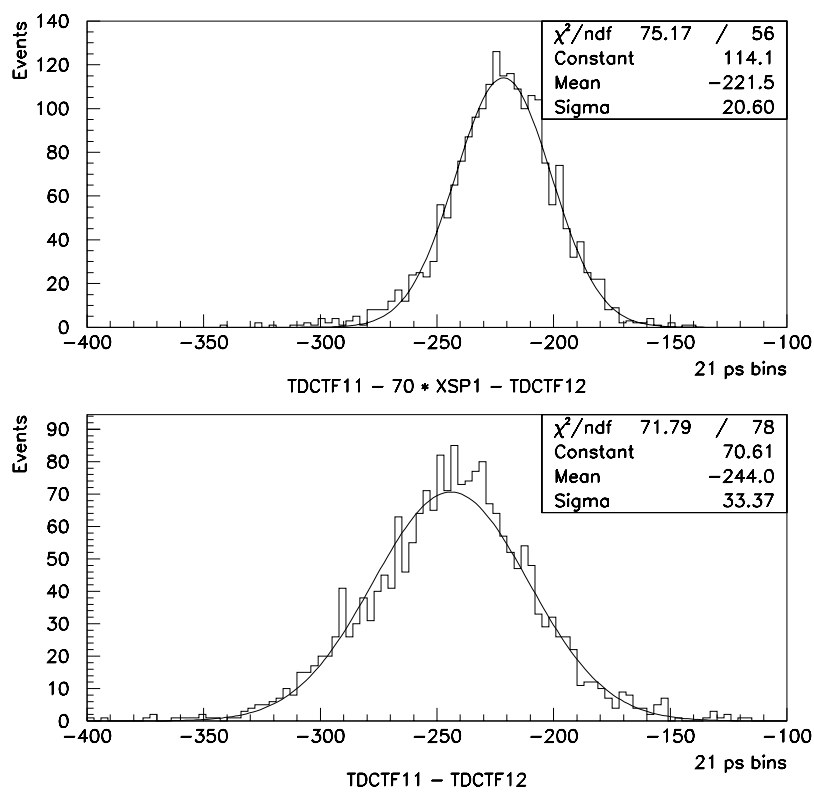

Figure 6.56: Timing resolution of $\mathrm{TF} 1$ with and without position dependence removed. The resolutions are $\sigma_{1}=0.44 \mathrm{~ns}$ and $\sigma_{1}=0.71 \mathrm{~ns}$ respectively. 


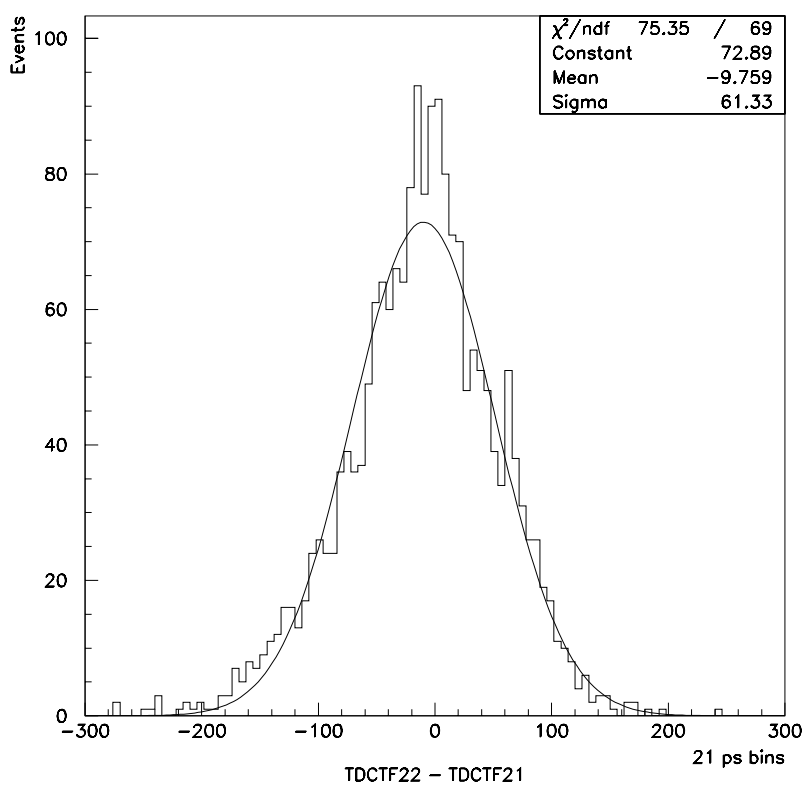

Figure 6.57: Timing resolution of $\mathrm{TF} 2\left(\sigma_{2}=1.3 \mathrm{~ns}\right)$.

flight normalized to the time of flight for $\beta=1$. Part of the spread in time of flight comes about because even at some fixed central beam momentum, the antiproton beam has a velocity spread related to its momentum spread, $\left(\delta p / p \leq 2 \times 10^{-4}\right)$.

The time of flight is calculated using the two methods as follows:

Average:

$$
\begin{array}{r}
\text { TOF }=((T D C T F 11+T D C T F 12) / 2- \\
(T D C T F 21+T D C T F 22) / 2) \cdot 0.0212 \mathrm{~ns} / \mathrm{LC} \cdot \beta+88.96 \mathrm{~ns}
\end{array}
$$

Corrected:

$$
\begin{array}{r}
\text { TOF }=(\text { TDCTF } 11-70 * X S P 1- \\
T D C T F 21+0.25 * Y S P 3) \cdot 0.0212 \mathrm{~ns} / \mathrm{LC} \cdot \beta+88.96 \mathrm{~ns}
\end{array}
$$



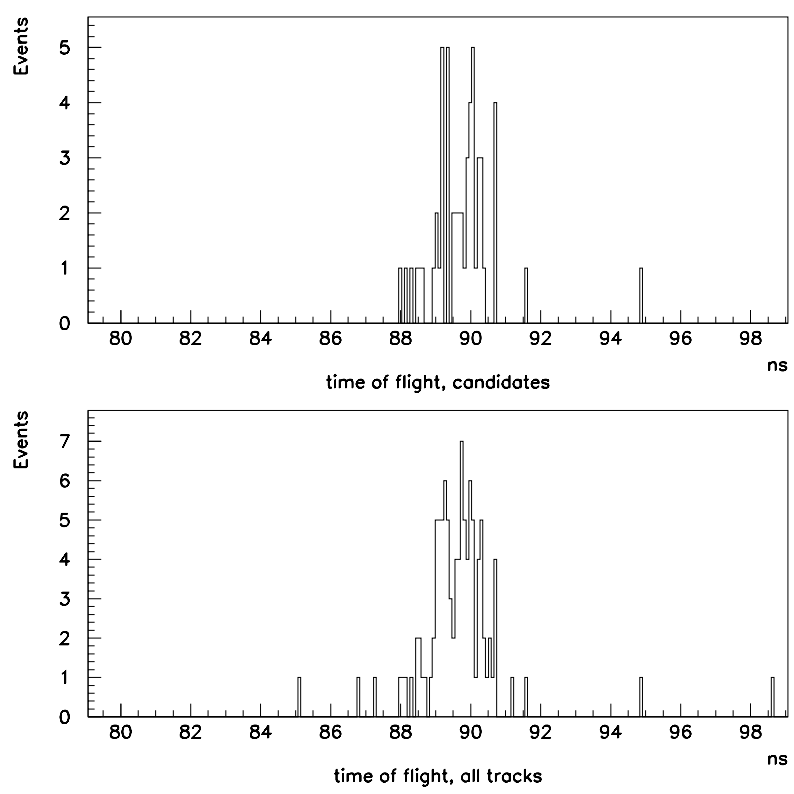

Figure 6.58: Time of flight distribution using method 1 , normalized to $\beta=1$. All events have good tracks with high momentum.

The distribution of the time of flight for the averaging method and the removal of correlations method are shown in figures 6.58, and 6.59. Approximate gaussian shapes are noticeable, but not much larger than that expected from the measured, individual counter resolutions $\sigma=\sqrt{\sigma_{1}^{2}+\sigma_{2}^{2}}=1.9 \mathrm{~ns}$. The top plot in each is the normalized time of flight for candidate events, the bottom plot is that for all tracks passing the selection criteria above. Clearly, the time of flight distributions show only one peak, meaning only one type of particle can be distinguished. This is consistent with the hypothesis that the dominant background in the antiproton spectrometer is antiprotons being lost from the beam. Accidental coincidences in the counters are distributed completely randomly in time. Most of these can be removed from the event sample.

Because of the low rate environment, most of the events passing the $T F 1 \otimes e^{+}$ trigger are expected to have hits in the two counters which are correlated in time (to within a couple of $\mathrm{ns}$ ). The time of flight difference between the two counters is about 

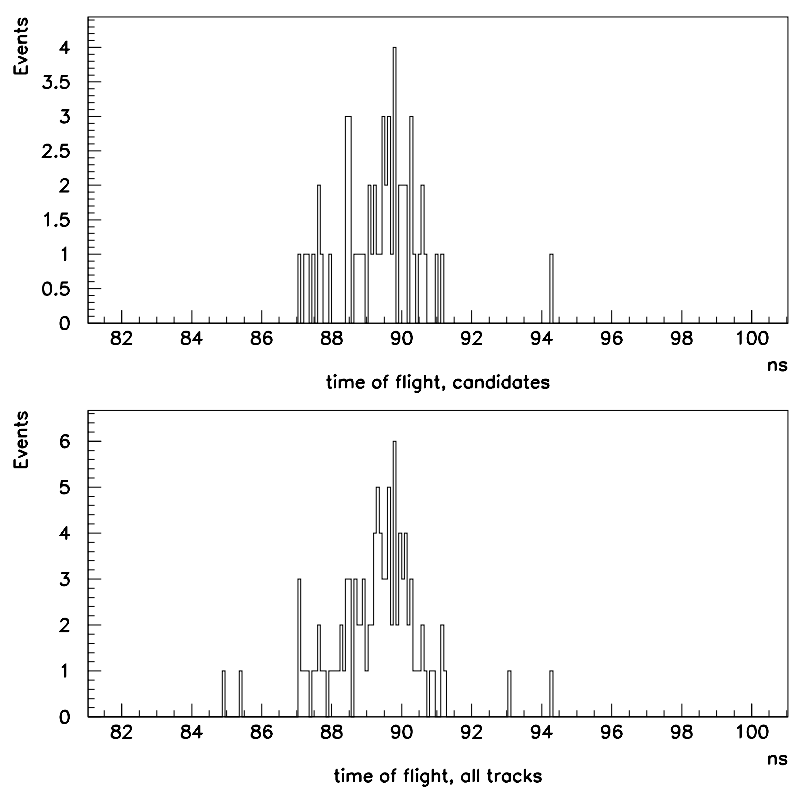

Figure 6.59: Time of flight distribution using method 2, normalized to $\beta=1$. All events have good tracks with high momentum.

6.5 ns. In figure 6.60 , one can see all the $T F 1 \otimes e^{+}$triggers showing most peak around $6.5 \mathrm{~ns}$ difference. A few are random hits perhaps of two unrelated antiprotons lost from the beam. The antihydrogen triple-coincidence candidates clearly are clustered around a $6.5 \mathrm{~ns}$ time difference. 

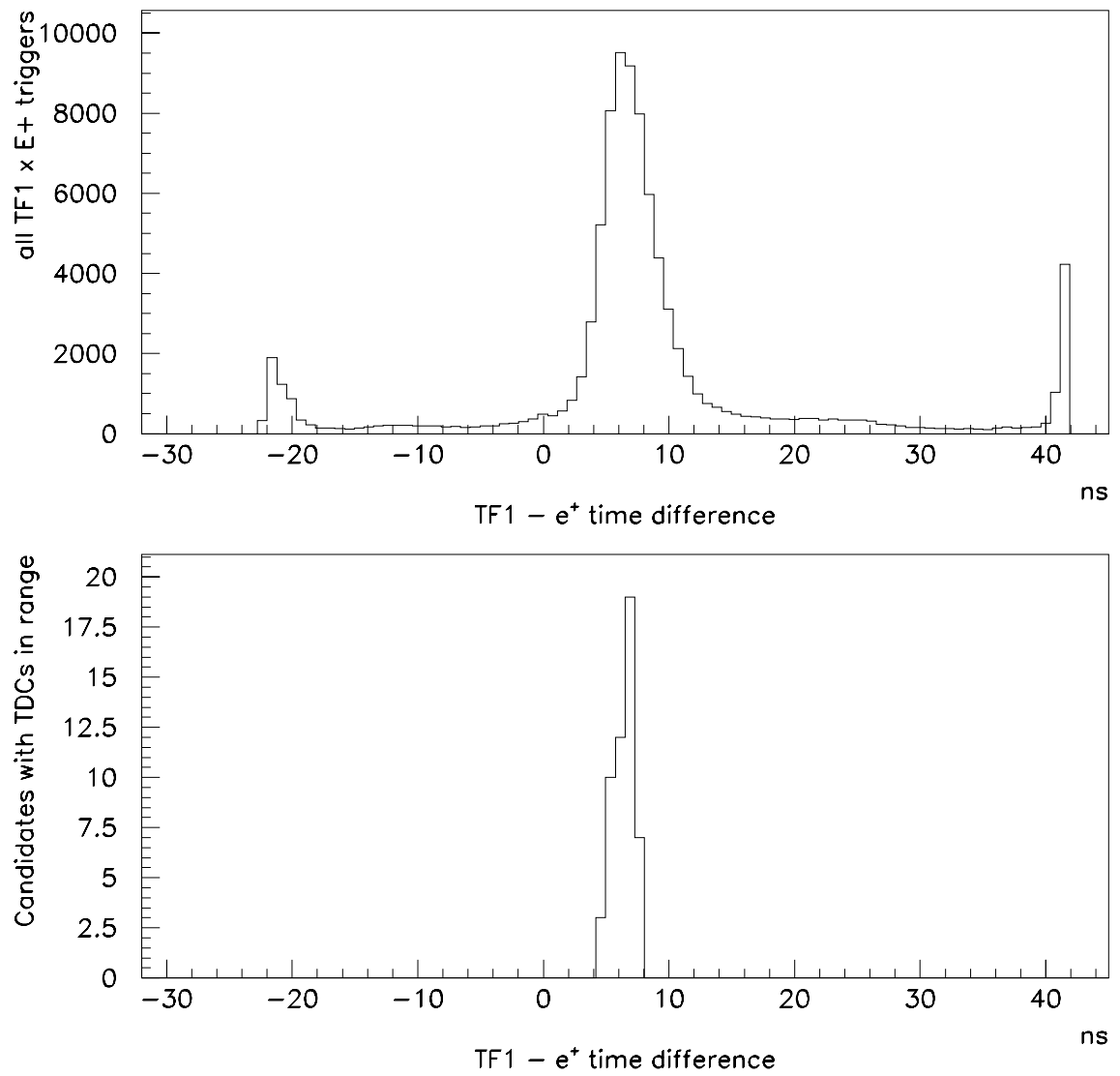

Figure 6.60: Time difference between TF1 and $e^{+}$for all $T F 1 \otimes e^{+}$triggers and candidate events. Only events from runs 21-115 were chosen. 


\subsection{Foil Target-Out data}

\subsubsection{Description of Foil-Out Data}

In order to really test the hypothesis that the triple coincidence events recorded were indeed antihydrogen, some data was taken with the foil target rotated out of the way, leaving a hole, $1 "$ in diameter. After run 50 , this hole was increased to $1.75 "$ in diameter. The wheel is $1 / 32$ " of aluminum. No triple coincidences were expected and none were observed. An antihydrogen that flies through the hole will also pass through the $e^{+}$dipole with no effect. It will ionize in the 0.002 " titanium vacuum window 3.25 inches upstream of PWC \#1. Because of the material, the positron will multiple scatter in $0.14 \%$ of $\mathrm{X}_{o}$ by $7^{\circ}$ at $p_{\bar{p}}=6 \mathrm{GeV} / \mathrm{c}$ (or $13 \mathrm{~mm}=13$ wires on average). Thus the positron and antiproton register separate hits in the wire chambers. The $e^{+}$still is usually within the acceptance of PWC \#1.

The first indication came in the first $5 \mathrm{pb}^{-1}$ of target-out data. Four events with beam momentum $\left(p_{\bar{p}}>0.995\right)$ tracks were seen. Three of them had at least one second hit in PWC \#1 in one of the planes. Some had a hit in each of all three planes of PWC \#1 that did not corresponding to the antiproton track. None had extra hits in the other two wire chambers. The data are therefore consistent with the antihydrogen hypothesis. If one expects that the events are not antihydrogen and the stripping foil has no effect, then the probability of getting no triple coincidences when one expects roughly five (based on the triple coincidence rate with foil in place) is $0.7 \%$. Also, if an antihydrogen cross section of $0.8 \mathrm{pb}$ is assumed, the probability of seeing three candidates out of $5 \mathrm{pb}^{-1}$ is $19.5 \%$. 


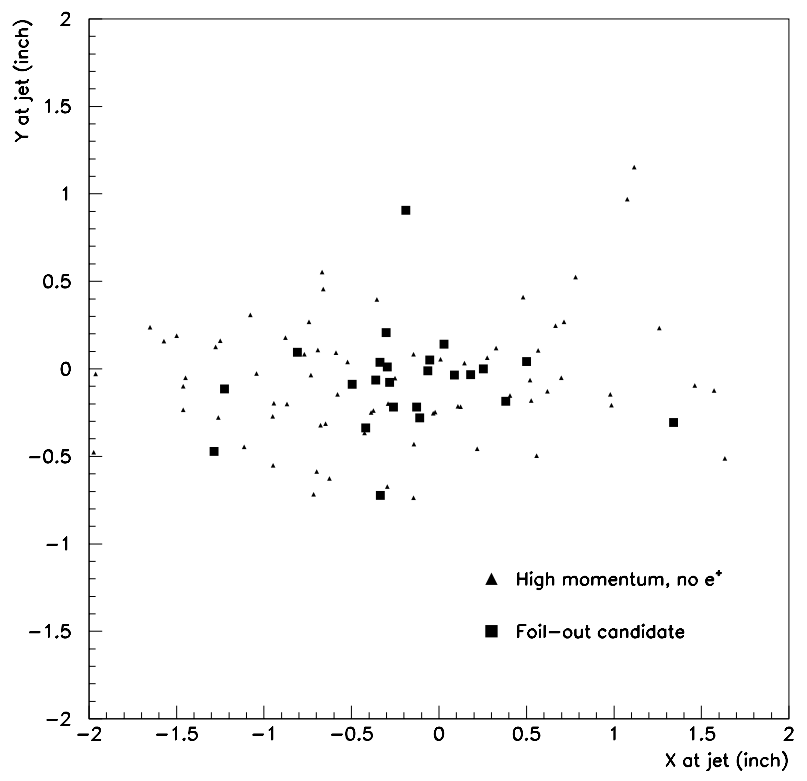

Figure 6.61: Transverse position of track at location of jet for foil-out antihydrogen candidates as well as beam momentum tracks without evidence of a $e^{+}$.

In all, 25 foil-out candidates were observed. Twenty-one had a beam momentum above transition in all of $33 \mathrm{pb}^{-1}$. The below transition, foil-out data corresponded to an integrated luminosity of $7 \mathrm{pb}^{-1}$.

\subsubsection{Jet Location}

Figure 6.61 shows the distribution of transverse positions of the antiproton tracks at the location of the jet for foil-out antihydrogen candidates as well as beam momentum tracks without an extra hit in PWC \#1. Figures 6.62, and 6.63 show the same distributions split up into above and below transition data respectively. 


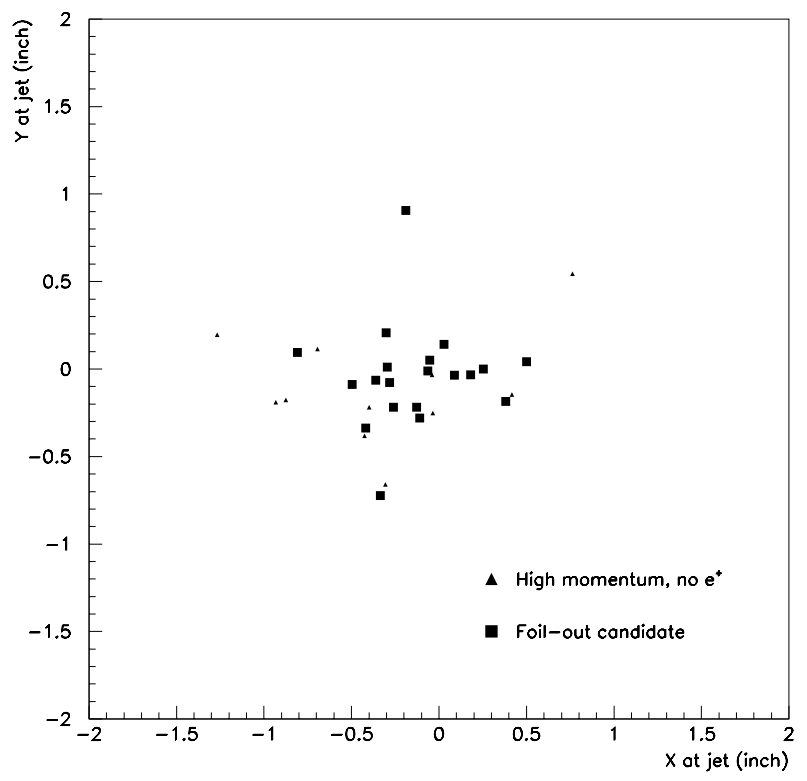

Figure 6.62: Transverse position of track at location of jet for foil-out antihydrogen candidates as well as beam momentum tracks without evidence of a $e^{+}$. Only above transition events are displayed.

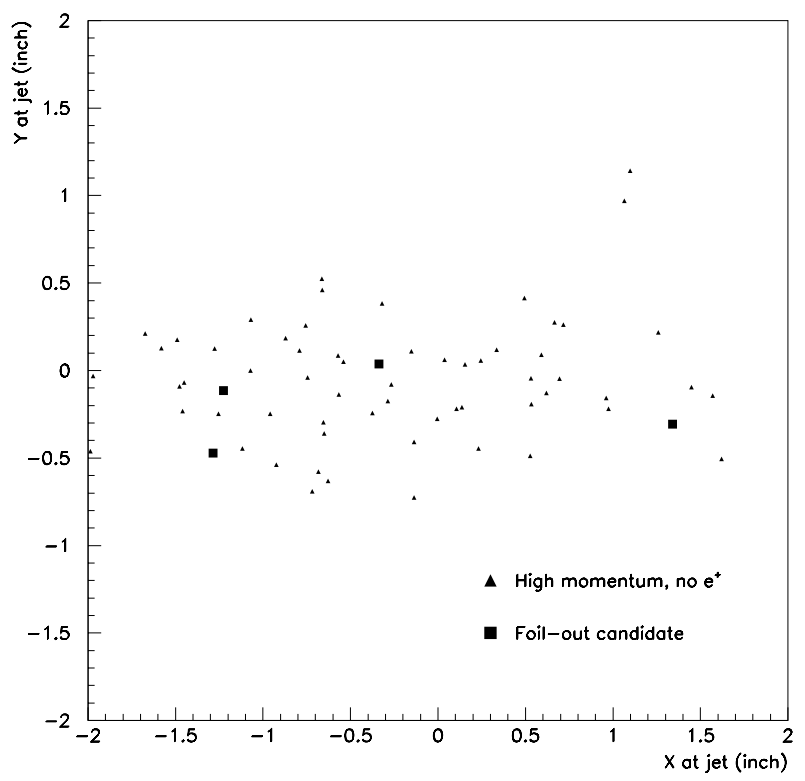

Figure 6.63: Transverse position of track at location of jet for foil-out antihydrogen candidates as well as beam momentum tracks without evidence of a $e^{+}$. Only below transition events are displayed. 


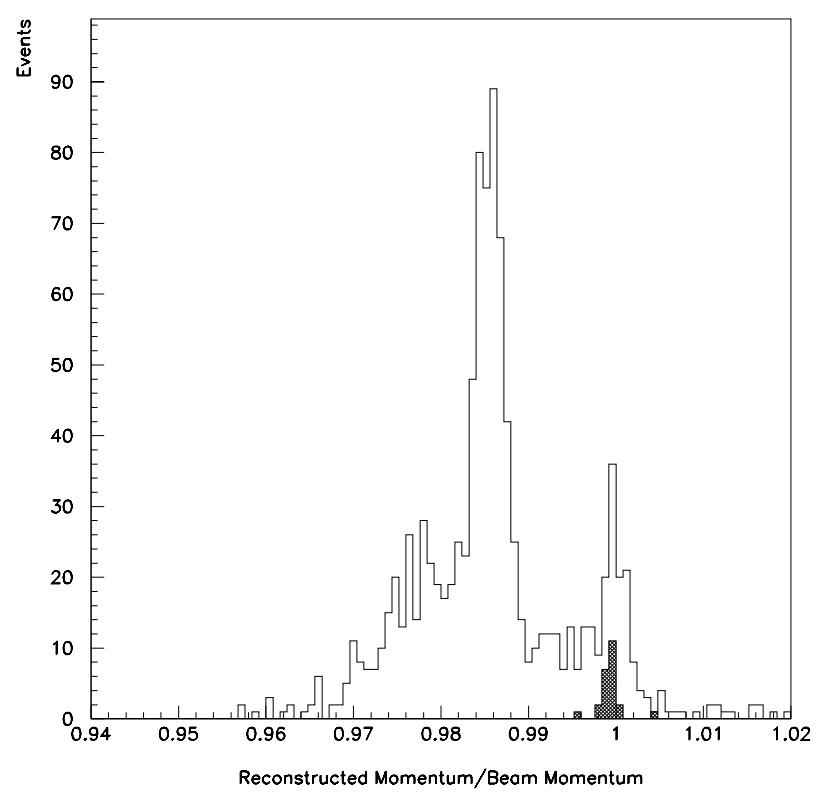

Figure 6.64: Fractional momentum distribution for foil-out candidates superimposed upon that for all events during foil-out running.

\subsubsection{Momentum Distributions}

Figure 6.64 shows the distribution of the ratio, reconstructed track momentum divided by beam momentum, for foil-out candidate events superimposed on the distribution for all events from the foil-out data both sets corresponding to above transition only. The candidate events are clustered around a fractional momentum of $\frac{p}{p_{\text {beam }}}=1.000$.

Figures 6.65 , and 6.66 show the distribution of the ratio, reconstructed track momentum divided by beam momentum for foil-out candidate events on top of the distribution for all events from the foil-out data, for above transition and below transition, respectively. Only four foil-out candidate events are shown in the below transition data. Only two are truly near a fractional momentum of $\frac{p}{p_{\text {beam }}}=1.000$. The other two are most likely, from the tail of the elastic scattering peak as discussed below. 


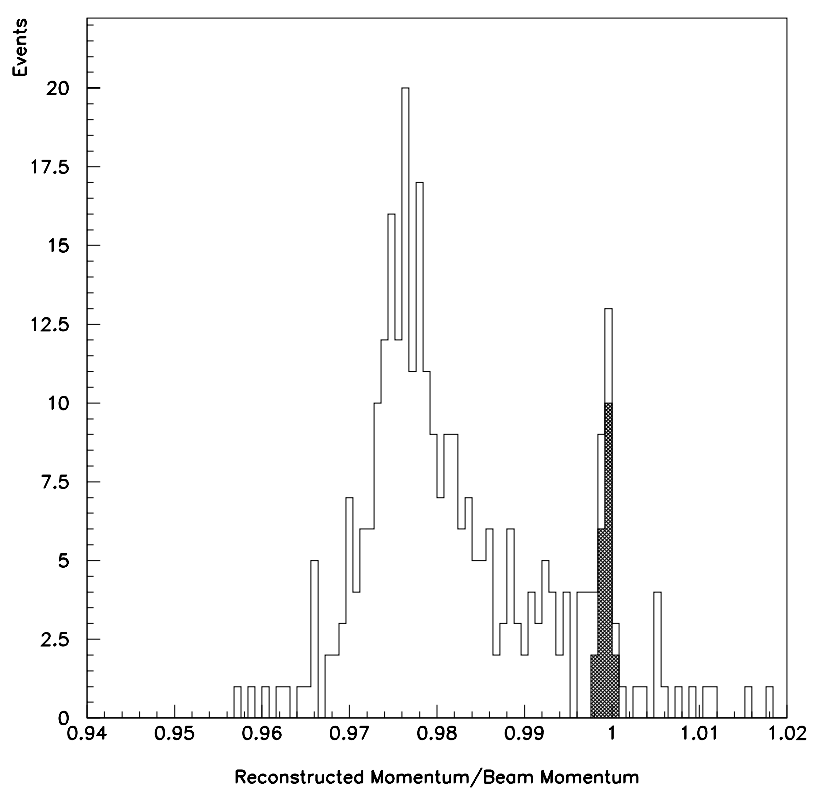

Figure 6.65: Fractional momentum distribution for foil-out candidates superimposed upon that for all events during foil-out running, both for above transition running.

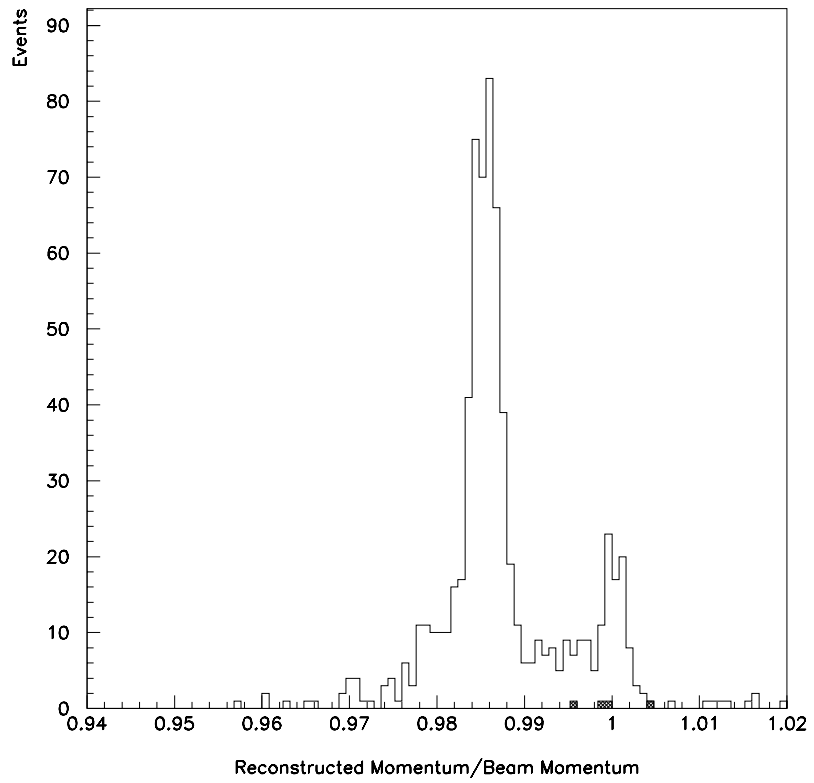

Figure 6.66: Fractional momentum distribution for foil-out candidates superimposed upon that for all events during foil-out running, both for below transition running. 
The reconstruction and unambiguous determination of the events involved separating out the correct track from the multiple tracks formed. All hits were paired to form possible space points in the wire chambers. If the spectator had hits in multiple planes, it could form a valid space point. If not, it might still pair with one of the antiproton hits to make an incorrect space point. Similarly, when the space points in PWC \#2 and PWC \#3 are considered to make a track, they might be associated with either the $e^{+}$space point or the space point formed from the incorrect pairing of $e^{+}$and $\bar{p}$ hits to give an incorrect track. These tracks are not extremely different from the true antiproton track in fractional momentum $(\sim \pm 0.003)$ but have much larger y residuals.

Below transition, only four foil-out candidates were observed. Only two are truly antihydrogen. The other two are most likely background from the tail of the elastic scattering distribution. Figure 6.66 shows the fractional momentum distribution for these events against a histogram of all events in the below transition, foil-out running. One notices the two antihydrogen events have fractional momentum close to 1.000 . The fractional momentum of the other two are different from 1.000. There is a possible systematic error from lack of knowledge of the proper shunt current for the antiproton dipole at each momentum. On the other hand, the location of the elastic scattering peak is almost a completely linear function of beam momentum. The elastic scattering peak location can be used to correct the fractional momentum of candidates. Because it is a small effect, it was not performed on all candidates. It was looked at especially for these events, though, in hope that the two background events had the momentum scale incorrect. However, after correction, the two events still remain about where they were in fractional momentum. In support of the background hypothesis, it should be noticed that both events had larger than usual $\mathrm{Y}$ residuals $\left(\left|Y_{\text {res }}\right|>0.2\right.$ inch). 
As in the foil-in data, there are beam momentum events without any evidence of a positron. Above transition, the ratio of these events to candidate events in the foil-out running is approximately 1:2 (actually 12:21). Below transition, the ratio is much larger (62:2) resulting from a larger beam hitting beampipe or steel. This should be compared to the foil-in ratios: above transition (31:61) and (63:3) for above and below transition respectively. The existence of a good track is assumed.

\subsubsection{Background to Foil-out Candidates}

There are three classes of events indicating a possible background for the antihydrogen candidates.

Two candidates having the foil-out signature show up in the foil-in data. These are interesting as a possible background to the foil-out antihydrogen candidate events. Only one event was above transition. The first event clearly shows two particles passing through the chamber separated by a few wires in each of 3 planes. The second lists adjacent wires in the first plane but plane 2 and 3 have separated wires on. Both of these suggest either an unstripped antihydrogen or a beam momentum antiproton kicking out an electron.

Two of the foil-out, below transition events are close to the candidates in fractional momentum and the number and placement of extra hits in PWC \#1. The fractional momentum and/or $\mathrm{Y}$ residual are different enough to exclude them as candidates but not by much.

Three of the triple coincidence events have extra hits in PWC \#1 (which are not adjacent hits). Of these, one has no $e^{+}$counter signal but one of the NaI halves registers a $511 \mathrm{keV}$ gamma ray. This one has hits which are all adjacent. It can therefore be excluded since there is no justification that the hits are from a separate 
particle. The second event has a strange timing scenario in which the hits associated with the antiproton are early and an extra hit in the first plane is a bit late. This and the third event are probably antihydrogen where the antiproton kicked off an atomic electron.

One possibility was that an antihydrogen found a hole in the carbon foil or that it traveled through the foil without ionization. The stripping is $>99 \%$. If it is equal to $99 \%$, the probability of observing one unstripped event out of 73 events (observed as candidates) is 0.35 and would be closer to one except for the uncertainty resulting from the statistics of small numbers.

Another possibility is that an antiproton can knock an orbiting electron out of a titanium atom in the vacuum window $3.25^{\prime \prime}$ upstream of PWC \#1. The electron will multiple scatter and lose some energy but if it gets out of the titanium, it may be seen as an extra hit in PWC \#1 (if it is within the acceptance). This can happen, of course, whether or not the antiproton results from an antihydrogen. The number of electrons per antiproton resulting from an antiproton of momentum $6 \mathrm{GeV} / \mathrm{c}$ with energy greater than $E$ is given roughly given by the ratio of $W / E$ where ( $x=$ thickness of window in $\mu \mathrm{g} / \mathrm{cm}^{2}$ ) [91],

$$
W(M e V)=\frac{0.15}{\beta^{2}} \frac{Z}{A} x=1.63 \mathrm{keV}
$$

The $e^{-}$must have enough energy to get out. A minimum ionizing electron would have lost only $37 \mathrm{keV}$ and the number of $e^{-}$with $E>37 \mathrm{keV}$ is equal to 0.044 . Since we require a beam momentum track, the number of beam momentum tracks, above transition, which could have knocked out an $e^{-}$is only about 100 . The number of expected background would thus be 4 . This is an extreme upper bound though since electrons with energies somewhat less than the rest mass lose substantially more energy. Electrons with kinetic energies around a couple of hundred keV lose start to lose a significant fraction of that energy so I have assumed $200 \mathrm{keV}$ to be 
Table 6.1: Interesting events with extra hits

\begin{tabular}{|c|c|c|}
\hline Run & Event & Frac. Mom. \\
\hline \multicolumn{3}{|c|}{ Below transition, foil-out } \\
\hline 61 & 919 & 1.000 \\
62 & 675 & 0.996 \\
64 & 1430 & 1.005 \\
115 & 226 & 0.998 \\
\hline \multicolumn{3}{|c|}{ Extra hit candidates in foil-out running } \\
\hline 70 & 1161 & 1.000 \\
72 & 2203 & 0.999 \\
\hline \multicolumn{3}{|c|}{ Triple coincidences with extra hits } \\
\hline 38 & 278 & 0.999 \\
40 & 1476 & 1.000 \\
81 & 2377 & 0.999 \\
\hline
\end{tabular}

the threshold for getting out of the titanium. Lower energy electrons are scattered more transversely and have less chance of being within the acceptance. For 100 beam momentum tracks, only 0.6 events of this type are expected. 


\subsection{Event Selection Summary}

Here are summarized, the cuts chosen to maximize the chance we were looking at antihydrogen and not background. The antihydrogen signature for the foil-in condition was background free. The zeroth order requirement of a triple coincidence in the trigger was enough to isolate the antihydrogens during normal running conditions. It is true that some data was inadvertently taken during high beam loss periods. The rate of triple coincidences went up significantly during these periods. The events were clearly not antihydrogen though. This was evident by the multitude of hits and tracks formed as well as by the random distribution of energy deposits in the positron and gamma ray detectors. These running periods were removed from the data used to determine candidates but served as a monitor of detector performance. The triple coincidence trigger events during normal running all either had one, reasonably good, near beam momentum track or did not have enough hits to form a track. Nearly all had a hit in the $e^{+}$scintillator with energy deposit close to that expected for antihydrogen.

The antihydrogen signature during foil-out running was more demanding. If the positron hit in PWC \#1 formed a space point with an unassociated hit in another plane (due to the antiproton), more than one track resulted. If one track had the momentum closest to beam momentum and the smallest $Y$ residual, the track was chosen as that of the antiproton. If not, the hits were examined by hand to determine which space points were being formed correctly. The extra hit in PWC \#1 had to be significantly separated from the antiproton hit. If they were less than 2 wires apart, the offline code would think they were an associated hit due to a particle passing in between two wires. As discussed in chapter 7, the inefficiency from this condition could be modelled. 
Table 6.2: Event selection criteria for antihydrogen candidates.

\begin{tabular}{|l|}
\hline Triple Coincidences \\
\hline $0.996<p / p_{\text {beam }}<1.002$ \\
$\left|Y_{\text {res }}\right|<0.15$ inch \\
Number of tracks $=0$ or 1 \\
$100<e^{+}$ADC $<450$ counts \\
Coincidence of all three triggers, not \\
$\quad$ just all three counters. \\
TF counter signal times consistent with beam particle \\
Energy deposit in either NaI half $<250$ counts \\
Transverse position at jet location consistent \\
with production in jet \\
Transverse position at foil location \\
inside foil radius \\
\hline
\end{tabular}

Table 6.3: Event selection criteria for antihydrogen candidates.

Foil-Out Candidates with Spectators

$0.996<p / p_{\text {beam }}<1.002$

$\left|Y_{\text {res }}\right|<0.15$ inch

Number of tracks $>0$

One clear, extra hit in at least one plane of PWC \#1

No energy deposits in $\mathrm{NaI}$

No energy deposit in $e^{+}$scintillator

$T F 1 \otimes T F 2$ trigger on but no others

TF counter signal times consistent with beam particle

Position at jet location consistent with

production in jet

Transverse position at foil location inside foil radius 


\section{Chapter 7}

\section{Geometrical Acceptance and Efficiencies}

\subsection{Simulation of foil-out candidates}

The geometrical acceptance $\times$ reconstruction efficiency for foil-out candidates was simulated with an EGS4(NRCC version) [92] Monte Carlo program. During the foil-out running, antihydrogen would ionize in the titanium vacuum window just before passing through the first wire chamber. The positron receives multiple Coulomb scatters while in the high $\mathrm{Z}$ titanium but is still expected, on average, to register a hit in PWC \#1. The antiproton leaves a high momentum track signature.

The simulation assumed the particle travels through a set of box-like regions in the longitudinal direction, The regions had infinite extent in the transverse direction. The thicknesses were 0.002 " of titanium, 3.25" of air, 0.001" kapton, 0.0005 " aluminum foil. A cut was made on the antiproton coordinates to make sure it did not hit a vacuum pipe or wire chamber aperture. In addition, the antihydrogen coordinates had to be consistent with passing through the foil. The position coordinates were then encoded into a format identical with real data. The offline reconstructed events from the simulated data. Those events that the offline determined would be 
high momentum events with spectator hits in PWC \#1 would define a reconstruction efficiency. Some positrons could be lost out of the edges of the PWC \#1 geometrical coverage defined by the outermost two instrumented wires in each plane. Only one hit is demanded, not a full space point. Most losses were actually from coalesced positron and antiproton hits, since only the last hit in time for any one wire was ever read out. For each region corresponding to a specific material, 50 steps were used to track the particle. For each run number-momentum combination during the fixed-target run, 500 events were simulated. The energy cutoffs used by the EGS4 program were $189 \mathrm{keV}$ for electrons and $10 \mathrm{keV}$ for photons. The tails of the angular distribution resulting from multiple scattering (Moliére distribution) were accurately reproduced. The simulation also simulated an occasional $(>1 \%)$ annihilation in the titanium resulting in two gamma rays as well as an occasional electron knocked out by the $e^{+}$.

Table 7.1 shows the resulting acceptances for different beam energies, position spreads and angular divergences. Clearly, the efficiency is over $90 \%$ except at very low energies (below the range of this experiment).

Figure 7.1 is a set of profiles of the positron and antiproton beam spots in the three wire chambers showing the outermost wire from each plane. The latest wire chamber configuration was used for all Monte Carlo generated acceptances.

Figure 7.3 shows the beam steering acceptance (the fraction of events which hit the foil) although it contains all aperture cuts on the antihydrogens as they travel towards the foil. This takes into account the acceptance loss of antiprotons downstream of the foil which primarily hit the beampipe near the large bend magnets. This is a small fraction of the overall acceptance though. The large dip in acceptance for the early runs was the result of the antiproton beam being misteered through the jet target. Sometimes it was an incorrect angle even though the beam intersected 

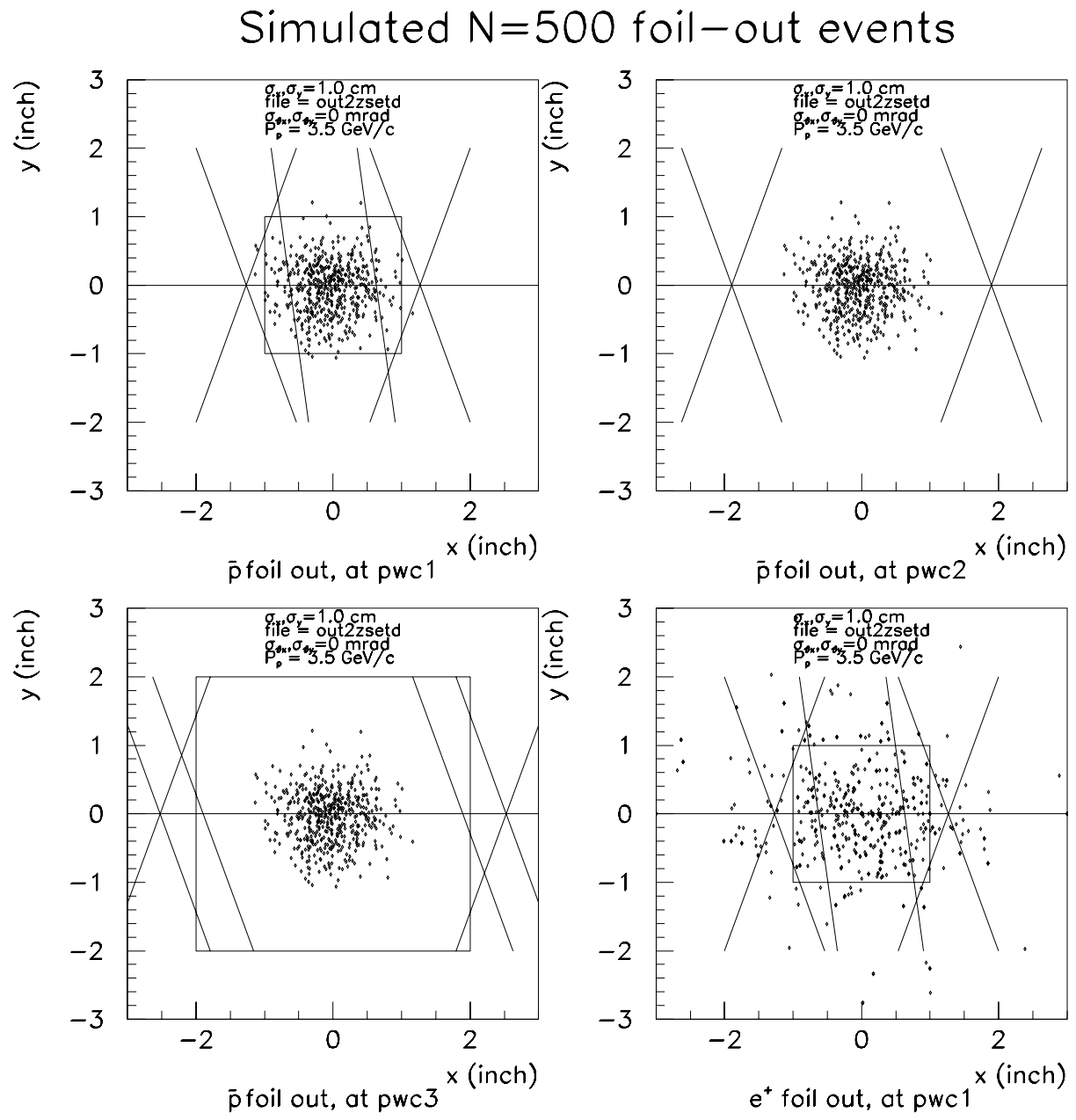

Figure 7.1: PWC profiles for $e^{+}$and $\bar{p}-$ set $\mathrm{d}$. 
Table 7.1: Efficiencies of foil-out events with spectators by Monte Carlo. $\varepsilon_{\text {high }}=$ efficiency of getting a high momentum track in offline for these events. $\varepsilon_{\text {extra }}=$ efficiency for getting a high momentum track and a spectator hit as seen by the offline.

\begin{tabular}{|c|c|c|c|c|c|c|c|c|c|}
\hline \#evts & $\begin{array}{c}\text { Momentum } \\
(\mathrm{GeV} / \mathrm{c})\end{array}$ & $\begin{array}{c}\sigma_{x} \\
(\mathrm{~cm})\end{array}$ & $\begin{array}{c}\sigma_{y} \\
(\mathrm{~cm})\end{array}$ & $\begin{array}{c}\delta_{x, y} \\
(\mathrm{~cm})\end{array}$ & $\begin{array}{c}\sigma_{\theta_{x, y}} \\
(\mathrm{mrad})\end{array}$ & $\begin{array}{c}\delta_{x, y} \\
(\mathrm{mrad})\end{array}$ & $\varepsilon_{\text {extra }}$ & $\varepsilon_{\text {geom }, \bar{p}}$ & $\varepsilon_{\text {high }}$ \\
\hline 500 & 6 & 0.5 & 0.5 & 0 & 0 & 0 & 0.970 & 0.998 & 1 \\
500 & 6 & 0 & 0 & 0 & 0 & 0 & 0.978 & 0.998 & 1 \\
500 & 6 & 1. & 1. & 0 & 0 & 0 & 0.962 & 0.998 & 1 \\
500 & 3.5 & 1. & 1. & 0 & 0 & 0 & 0.910 & 0.994 & 1 \\
500 & 3.5 & 0 & 0 & 0 & 0 & 0 & 0.934 & 1.0 & 1 \\
500 & 3.5 & 0.5 & 0.5 & 0 & 0 & 0 & 0.932 & 1.0 & 1 \\
500 & 5.5 & 0.5 & 0.5 & 0 & 0 & 0 & 0.964 & 1.0 & 1 \\
500 & 6.5 & 0.5 & 0.5 & 0 & 0 & 0 & 0.980 & 1.0 & 1 \\
10000 & 6 & 0.5 & 0.5 & 0 & 0 & 0 & 0.965 & 0.958 & 1 \\
500 & 6 & 0.5 & 0.5 & 0 & 0.25 & 0 & 0.970 & 0.998 & 1 \\
500 & 6 & 0.5 & 0.5 & 0 & 1.25 & 0 & 0.962 & 0.848 & 0.991 \\
500 & 3.5 & 0.5 & 0.5 & 0 & 0.25 & 0 & 0.932 & 1.0 & 1 \\
500 & 3.5 & 0.5 & 0.5 & 0 & 1.25 & 0 & 0.925 & 0.848 & 0.988 \\
500 & 1.5 & 0.5 & 0.5 & 0 & 0.25 & 0 & 0.496 & 0.976 & 1 \\
\hline
\end{tabular}

the jet. E862 was much more sensitive to the angle than E835. Figure 7.2 shows the steering acceptance as a function of the distance of the beam centroid from the center of the foil (or hole). Both foil types used in the experiment are considered. Gaussian distributed beam profiles are assumed with three beam sizes, (at $1 \sigma)$, of $(0.25 \mathrm{~cm}$, $0.50 \mathrm{~cm}, 0.75 \mathrm{~cm})$.

Figure 7.4 shows the reconstruction efficiency for foil-out events. The abscissa in these plots is not run number but a combination of run number and beam momentum since certain runs had multiple momenta. Some systematic error will arise from emittance and beam position monitor measurement errors, incorrect setting of the target wheel angle, and bowing of the titanium window. These are summarized in section 8.4 . 


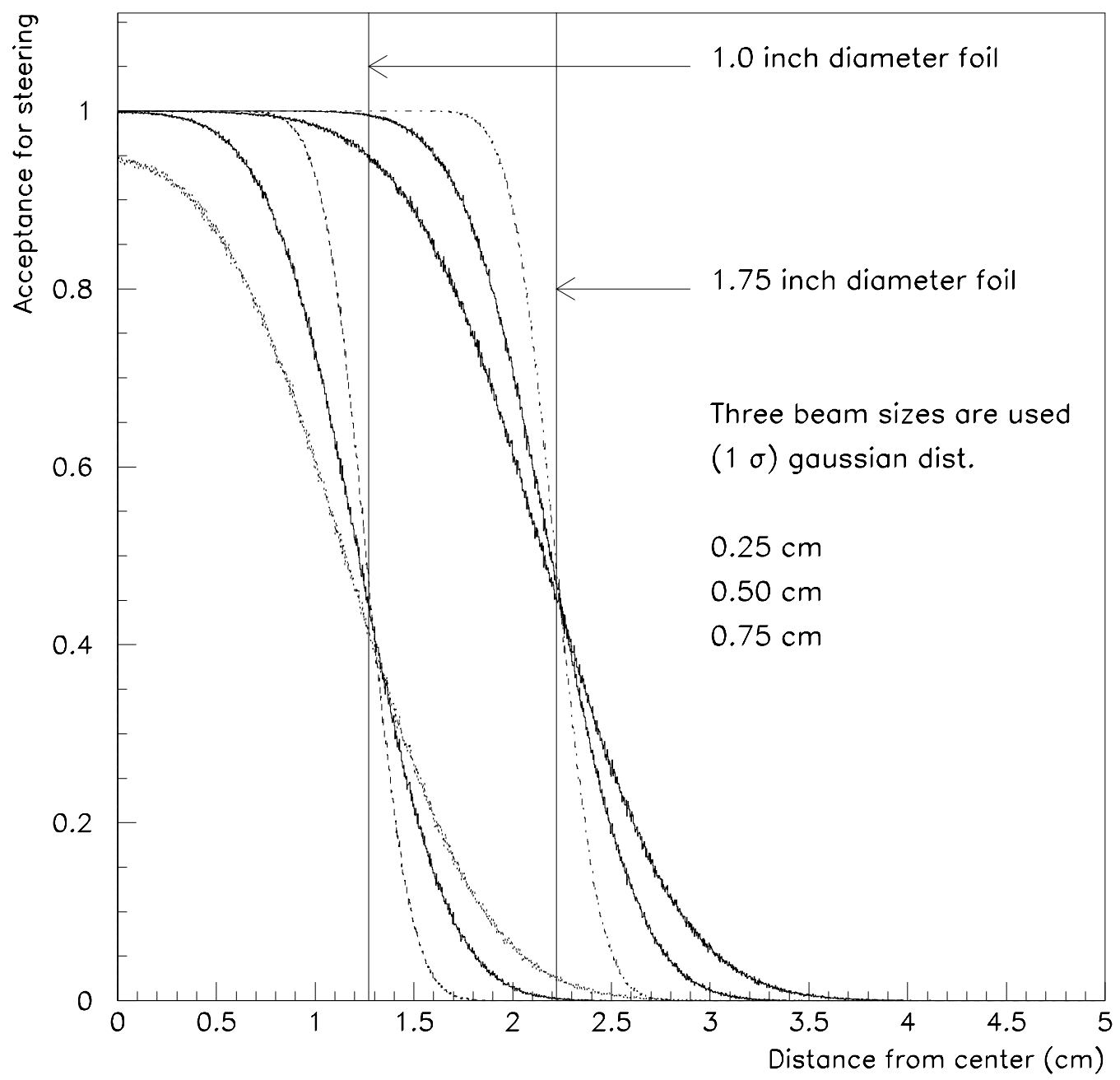

Figure 7.2: Steering acceptance as a function of distance from the beam centroid to the center of the foil aperture. 


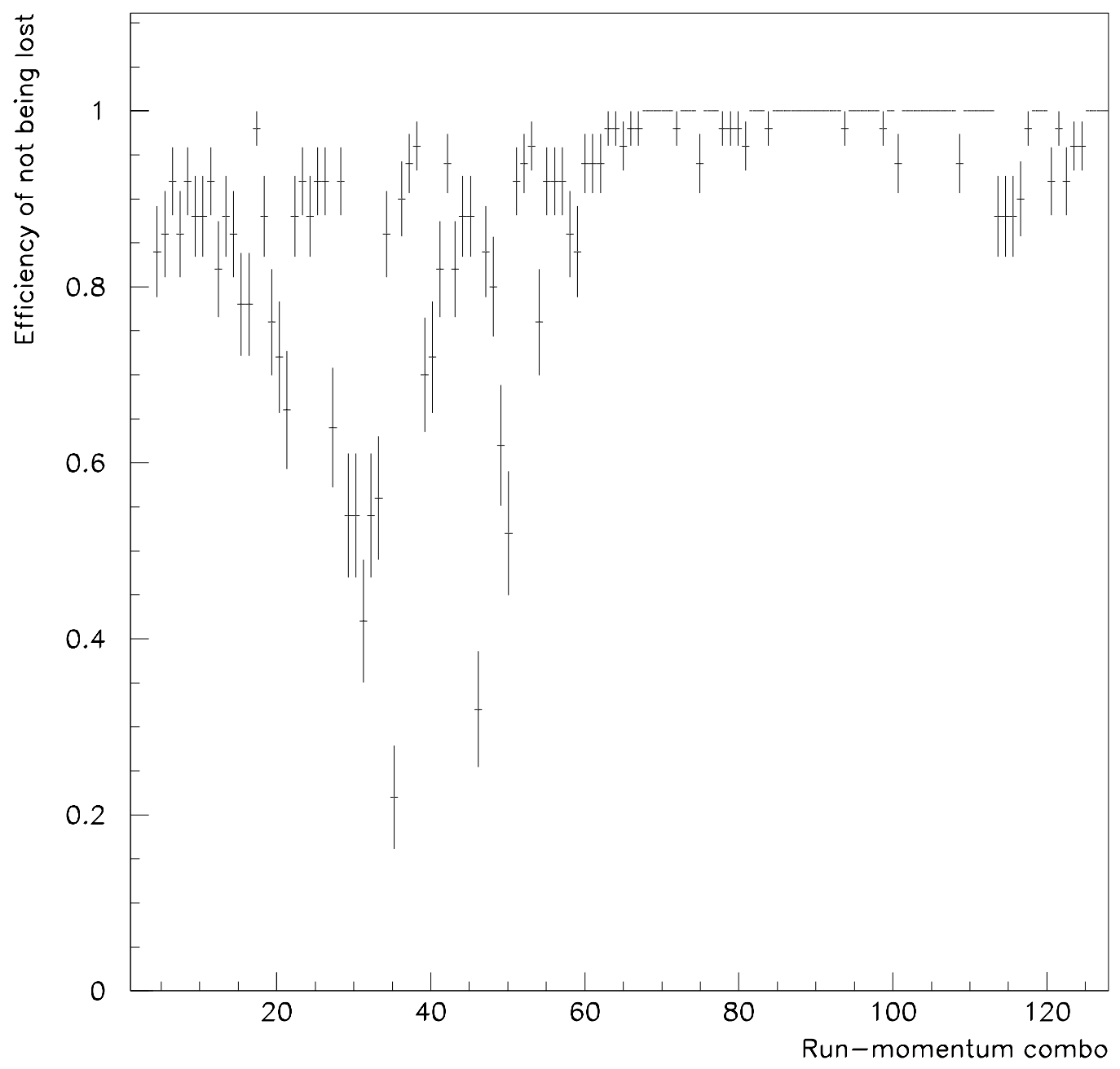

Figure 7.3: Acceptance losses for antihydrogen on apertures. The abscissa is not E862 run number but a combination of run number and momentum since some runs had more than one momentum. 


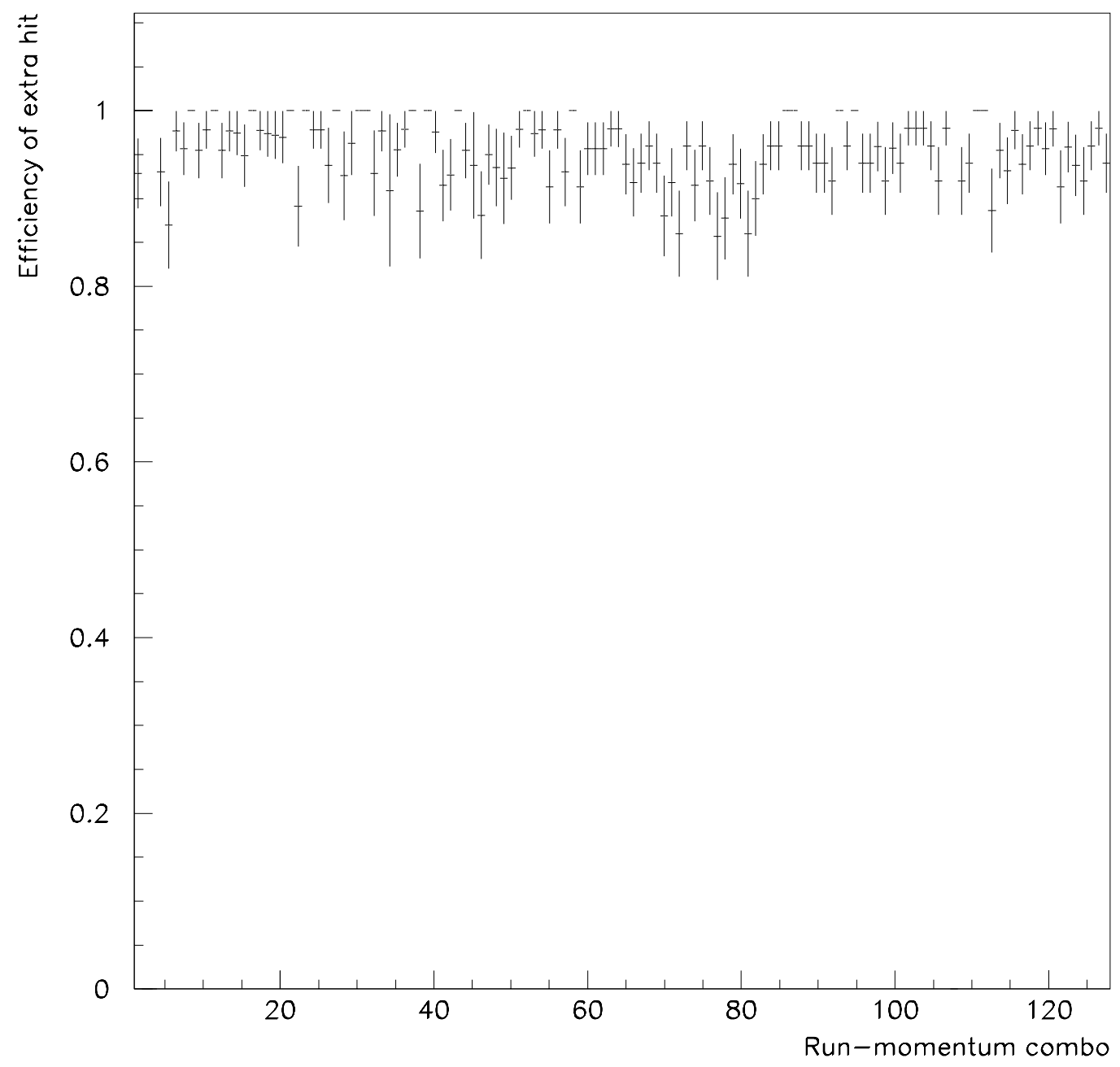

Figure 7.4: Acceptance $\times$ reconstruction efficiency for missing the positron. The abscissa is not E862 run number but a combination of run number and momentum since some runs had more than one momentum. 


\subsection{Simulation of positron beamline}

In order to understand the effects of the magnetic fields on the trajectories of the positrons through the magnetic spectrometer, several Monte Carlo simulations were built. I describe here one based on the transverse phase space (coordinates and angles) of the $e^{+}$. This is valid since only magnets are considered and energy is conserved. A transport matrix method was used to initially model the trajectories and the beam spot at the location of the scintillator. Eventually, a simulation involving magnetic fieldmaps was developed. The force on the particles was integrated using a Runge-Kutta integration routine.

Geometrical acceptances are presented for antihydrogen candidates which arrived on the $T F 1 \otimes e^{+}$trigger during target foil-in running. The geometrical acceptance is calculated by swimming positrons through a simulation of the positron spectrometer magnetic fields.

The E862 spectrometer performs two essential functions. First, it bends the positron out of the way of the antiproton's nearly straight trajectory by $40^{\circ}$. Second, a set of solenoids focuses the angular distribution of positrons resulting from multiple scattering in the carbon target foil. The solenoid magnets are run by one power supply in series. This limits the flexibility one has in adjusting the currents. On the other hand, the system is supposed to provide a one-to-one focus. In order to keep the image spot from rotating in the image plane (transverse to the beam direction at the scintillator location), the two solenoids should have field directions opposite from each other.

There are two solenoid applied current values which cause fundamentally different trajectories. The lower field "tune" has a larger focal length and the trajectories 
have their widest transverse extent within the dipole. This tune was used for the magnet current - momentum calibration using the ${ }^{207} \mathrm{Bi}$ monoenergetic electron source, since the momentum acceptance in the dipole is smaller. The higher field tune is strong enough to make the trajectories cross in the center of the dipole creating a second focal point halfway in between. More particles are accepted because they are closer to the central trajectory than with the high dispersion tune. This low dispersion tune was used exclusively for antihydrogen running because the acceptance was better.

When the magnets were installed in the ideal locations, it was found the beam spots were not centered. The locations of the magnets had to be changed in order to center the beam for both tunes. The final configuration found the solenoids with fields in the same direction and the magnet centers displaced in the vertical direction by $+3 / 32$ inch for the first solenoid and $-3 / 32$ " for the second. The dipole was displaced by $-1.19 \mathrm{~cm}$ in $\mathrm{x}$ (towards the accumulator) and $-3.18 \mathrm{~cm}$ in $\mathrm{z}$ (upstream). The object of this Monte Carlo was to accurately model the acceptance of the system under real conditions.

\subsubsection{Transport Matrices}

A rough estimate of the performance of the spectrometer can be found by using transport matrices acting on the transverse phase space $\left(x, y, x^{\prime}, y^{\prime}\right)$, along the axis of the central trajectory of the system. Hereafter, $\mathrm{y}$ is the vertical direction, and $\mathrm{z}$ is, in most cases, the direction of the beampipe (usually the central trajectory as well), while $\mathrm{x}$ is also in the horizontal plane normal to $\mathrm{z}$. The solenoids can be modelled with a focal length equal in both $\mathrm{x}$ and $\mathrm{y}$ because of their cylindrical symmetry. The dipole can be modelled by a horizontal focal length for a thin lens situated in the 
center of the dipole as well as a vertical focal length for thin lenses at the fringe field locations with a drift length in between. Apertures can be checked during drift distances.

The acceptance is determined by counting the number of positrons which are left within the radius of the scintillator in the image plane.

The transport matrix method can be used for zeroth order calculations of focusing. Here the impulse approximation is used to model a magnet (solenoid or multipole) as an operator on the phase space of a charged particle. The individual elements of the beamline are as follows:

\section{- Drift distance L}

$$
\left(\begin{array}{ll}
1 & L \\
0 & 1
\end{array}\right)
$$

\section{- Solenoid}

The solenoid can be modelled by a focus in both dimensions:

$$
\left(\begin{array}{cc}
1 & 0 \\
-1 / f_{s} & 1
\end{array}\right)
$$

where

$$
f_{s}=-\left.r \frac{d z}{d r}\right|_{z=L}=L_{o} / \tan \left(L / L_{o}\right)
$$

and

$$
L_{o}=2 p c / q B_{o}=\frac{2 p[M e V / c]}{3 \times 10^{-4} B_{o}[G]}
$$

\section{- Sector Dipole}

This sector dipole has flanged edges which use the fringe field to match the 
net horizontal and vertical focal lengths for the positrons. In horizontal phase space, the lens with focal length $f_{h}$ can be located at the center of the magnet. In the vertical phase space, the lenses are located at the entrance and exit of the magnet.

$$
\begin{aligned}
& \left(\begin{array}{cc}
1 & L / 2 \\
0 & 1
\end{array}\right) \cdot\left(\begin{array}{cc}
1 & 0 \\
-1 / f_{h} & 1
\end{array}\right) \cdot\left(\begin{array}{cc}
1 & L / 2 \\
0 & 1
\end{array}\right) \\
& \left(\begin{array}{cc}
1 & 0 \\
-1 / f_{v} & 1
\end{array}\right) \cdot\left(\begin{array}{cc}
1 & L \\
0 & 1
\end{array}\right) \cdot\left(\begin{array}{cc}
1 & 0 \\
+1 / f_{v} & 1
\end{array}\right)
\end{aligned}
$$

Figures 7.5, and 7.6 show some initial results using the matrix method. The reproduction of the object shape in the image plane at the location of the scintillator can be seen in the second figure. These were done with the ideal setup of the system without shifts in the magnet positions.

\subsubsection{Magnet Fields}

A more accurate simulation involves swimming the positrons through magnetic fieldmaps. A random, gaussian distribution of positron coordinates (angles, and momenta) is generated based on input parameters. As the particle travels through the spectrometer, a fourth order Runge-Kutta routine integrates the force on the particle to get the new coordinates and momenta. Whenever a value of magnetic field is needed at a point in space, a routine is called which contains (overlapping) fields for the different magnets. The positions of the magnets in all three dimensions can be changed slightly to simulate the effect on the $e^{+}$beam. 

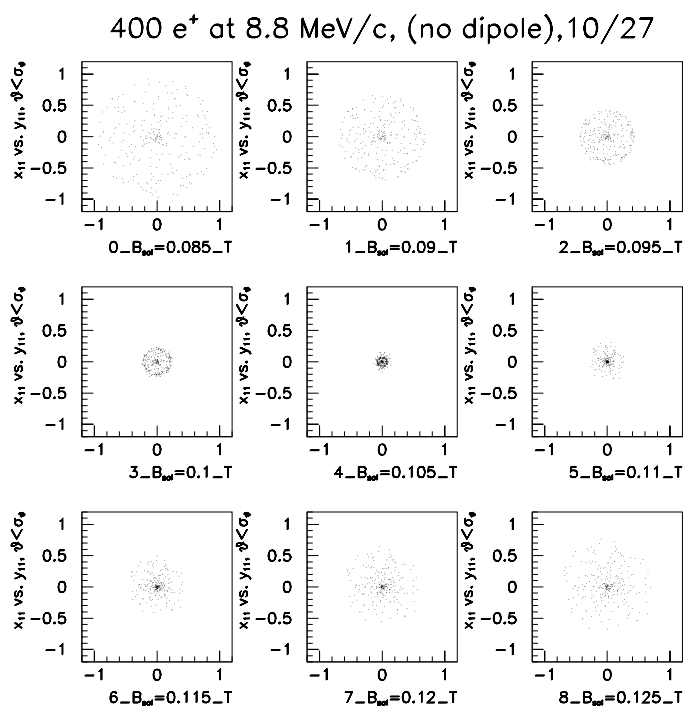

Figure 7.5: Simulated positron beam profiles at the scintillator for different magnetic fields. A field of $1.05 \mathrm{kGauss}$ shows the best focusing.

$5 \mathrm{~K} \mathrm{e} \mathrm{e}^{+}$at $5.5 \mathrm{MeV} / \mathrm{c}, 0.12,0.04 \mathrm{~T}, 10 / 26, \mathrm{~A}$
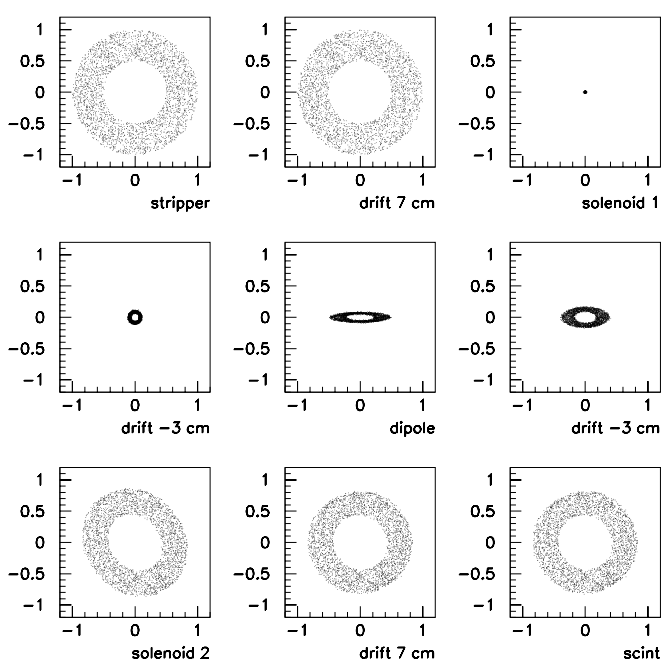

Figure 7.6: Simulated optics of a ring image through the positron magnets. 
The coordinate system used for the simulation is similar to the coordinate system used in the matrix method described above in section 7.2.1. The latter coordinates are the difference in transverse position and angle from the central trajectory. This works well for the solenoid. The dipole makes the central trajectory bend, though, and would thus introduce a non-cartesian coordinate system. So as not to introduce artifacts that might not be easily understood, the straight line defining the middle of the ideal beampipe is used as the $\mathrm{z}$ axis up until the particle crosses the symmetry axis of the dipole. After crossing this line, a similar straight line at an angle of $40^{\circ}$ but still in the $\mathrm{x}-\mathrm{z}$ (horizontal) plane serves as a new $\mathrm{z}$ axis. The plots showing the particle's $\mathrm{x}$ position (as a function of $\mathrm{z}$ ) show a cusp in the center where the dipole is located. This is just the sagitta. When the particle crosses the symmetry axis of the dipole, ideally (with enough steps in the simulation), the $\mathrm{x}$ position remains unchanged. The $\mathrm{z}$ location changes. With respect to the $\mathrm{z}$ of the symmetry axis in either straight line coordinate system, the z-location of the particle changes sign when it crosses this line. This explains the small gap in $z$ values seen in the plots.

The solenoid field is read from a design fieldmap, (see figure 7.7), containing 336 measurements at steps of $0.219 \mathrm{~cm}$. This map agrees well with one measured with the Hall probe, (see figure 3.14). It includes the longitudinal field $\left(B_{z}\right)$ along the $z$ axis through the fringe field regions. The transverse fields are generated from the longitudinal field using $\vec{\nabla} \cdot \vec{B}=0$ throughout and $\vec{\nabla} \times \vec{B}=0$ only near the central axis.

The dipole field is found from symmetry. The coordinate system is rotated into the symmetry coordinate system of the dipole. It is determined if the particle is within the area under the steel or is in the fringe field region. If in the fringe field region, a further transformation is made to align the coordinate system with the 


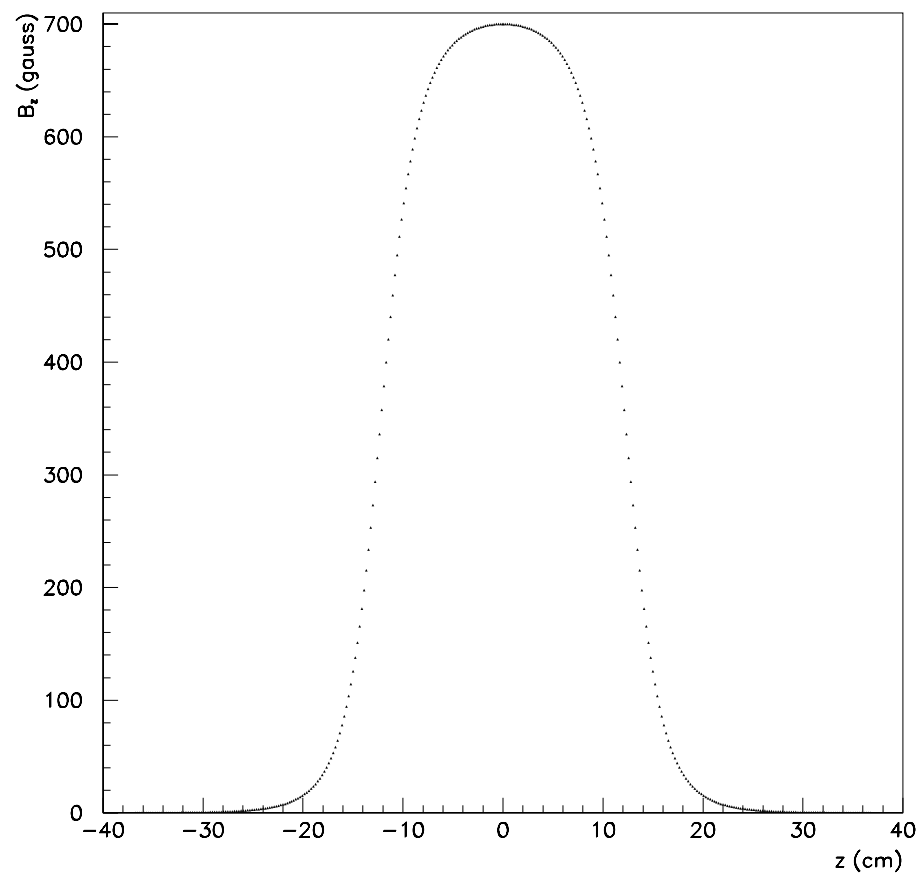

Figure 7.7: Simulated solenoid longitudinal magnetic field, $B_{z}(z)$ along longitudinal direction. 


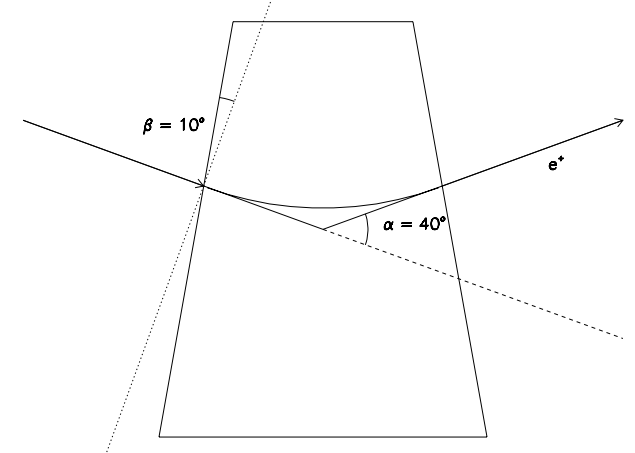

Figure 7.8: Diagram of the sector dipole magnet showing the flanged edges for focusing.

flanged edges of the steel. One quarter wave of a cosine function is attached smoothly to the boundaries. The field components then undergo the reverse coordinate system transformations to bring them back to the coordinate system used as input to the routine.

1. Region I - incoming fringe field (tilted $-10^{\circ}$ w.r.t. the dipole symmetry system)

$$
\begin{array}{r}
B_{y}=-\frac{B_{y o}}{2} \cosh \left(\frac{\pi y}{z_{2}-z_{1}}\right) \cdot \cos \left(\frac{\pi\left(z-z_{1}\right)}{z_{2}-z_{1}}\right)+\frac{B_{y o}}{2} \\
B_{z}=-\frac{B_{y o}}{2} \sinh \left(\frac{\pi y}{z_{2}-z_{1}}\right) \cdot \sin \left(\frac{\pi\left(z-z_{1}\right)}{z_{2}-z_{1}}\right)
\end{array}
$$

2. Region II - Steel (in the dipole symmetry coordinate system)

$$
B_{y}=B_{y o}, B_{x}=B_{y}=0
$$

3. Region III - outgoing fringe field (tilted $10^{\circ}$ wrt the dipole symmetry system)

$$
\begin{array}{r}
B_{y}=-\frac{B_{y o}}{2} \cosh \left(\frac{\pi y}{z_{2}-z_{1}}\right) \cdot \cos \left(\frac{\pi\left(z_{2}-z_{1}+z_{3}-z\right)}{z_{2}-z_{1}}\right)+\frac{B_{y o}}{2} \\
B_{z}=-\frac{B_{y o}}{2} \sinh \left(\frac{\pi y}{z_{2}-z_{1}}\right) \cdot \sin \left(\frac{\pi\left(z_{2}-z_{1}+z_{3}-z\right)}{z_{2}-z_{1}}\right)
\end{array}
$$


Table 7.2: Input values to spectrometer simulation.

\begin{tabular}{|c|c|}
\hline Total length of beamline & $181.281 \mathrm{~cm}$ \\
Stripper foil defines & $0 \mathrm{~cm}$ \\
Position of solenoid 1 & $45.00 \mathrm{~cm}$ \\
Position of dipole & $90.64 \mathrm{~cm}$ \\
Position of solenoid 2 & $135.64 \mathrm{~cm}$ \\
Length of solenoid fieldmap & $73.56 \mathrm{~cm}$ \\
Length of solenoid & $21 \mathrm{~cm}$ \\
Length of dipole steel at central trajectory & $21.5138 \mathrm{~cm}$ \\
Length of each dipole fringe field region & $\sim 1.75 \mathrm{~cm}$ \\
\hline
\end{tabular}

The important parameters used in the ideal simulation (without magnet offsets, etc) are listed in table 7.2.2:

The ideal fieldmap for the dipole on axis is displayed in figure 7.9 for 2000 divisions along the axis. The fringe field length is chosen to so that the total $\int B d L$ gives perfect bending.

The spread in the initial angular distributions are caused by the initial antiproton beam spread (the antihydrogen has almost exactly the same beam parameters as the antiproton beam at the gas jet target location) and the multiple scattering from passing through the carbon foil. For the multiple scattering mean angle, the Moliére formula is used[13]:

$$
\theta_{M S}=\frac{13.6 M e V}{p \beta c} \sqrt{\frac{x}{X_{0}}}\left(1+0.038 \cdot \ln \left(\frac{x}{X_{0}}\right)\right)
$$

For case studies of the spectrometer's focusing abilities, the input beam parameters such as antiproton momentum, position, and angle are used as centroids. Beam size, divergence, and momentum spread are input and used to throw gaussian distributions in the kinematic variables of a trajectory. Presently, two fixes had to be entered to adjust the field strengths from what they were originally. These were set to provide the best capabilities at the maximum momentum setting. The dipole 


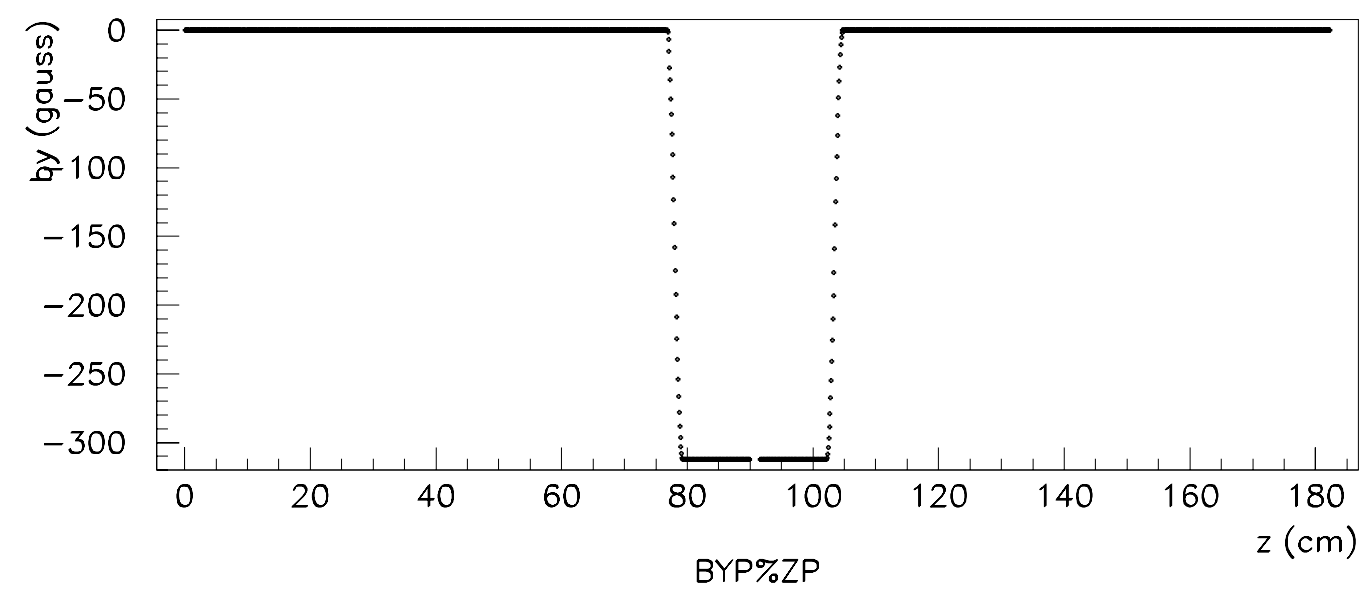

Figure 7.9: Ideal on z-axis magnetic field strength, $B_{y}$.

strength was multiplied by 0.9875 to bend the positrons perfectly. The solenoids fields were found by linearly scaling one of the maximum field settings at about $6 \mathrm{GeV} / \mathrm{c}$ antiproton momentum. The field was multiplied by a normalized fieldmap described above. The solenoid field was multiplied by a factor of 1.02 to get perfect focusing.

The ideal configuration of the magnets can be studied with a point beam. Figures 7.10, 7.11, and 7.12 show the tracking of $e^{+} \mathrm{s}$ (or $e^{-} \mathrm{s}$ ) for the low dispersion, plus-minus tune with the ideal magnet positions for three momenta corresponding to antiproton momenta of $3,6,8.85 \mathrm{GeV} / \mathrm{c}$ respectively. The notation "pm" or plusminus denotes the sign of the dot product of magnetic field of each solenoid with the particle's momentum vector (times electric charge) $(\operatorname{sign}(q \vec{B} \cdot \vec{p}))$. Figure 7.13 shows the tracking for the high dispersion, pm tune at $6 \mathrm{GeV} / \mathrm{c}$. Since most of the measured antihydrogen data is at $6 \mathrm{GeV} / \mathrm{c}$ antiproton momentum and all of that data is using the low-dispersion tune, the following case studies will be shown only for this case. The solenoids, dipole fringe fields, and entire dipole could be turned off to observe the effect on the particle trajectories. 
Table 7.3: Beam offsets and spreads (units $=\mathrm{cm}$ ).

\begin{tabular}{|c|c|c|c||c|c|}
\hline $\mathrm{X}$ offset & $\mathrm{Y}$ offset & $\mathrm{X}$ spread & Y spread & Figure & Acceptance \\
\hline 1 & 0 & 0 & 0 & 7.14 & 0.98 \\
0 & 0 & 1 & 0 & 7.15 & 0.88 \\
1 & 1 & 1 & 1 & 7.16 & 0.44 \\
\hline
\end{tabular}

Figures $7.14,7.15$, and 7.16 are simulations where the magnets are in the ideal configurations but the positron beam is either offset or spread out by $1 \mathrm{~cm}$ in either or both directions.

Simulations were done with different magnet offsets. Once case, shown in figure 7.17, has a dipole moved from its ideal position by $1.19 \mathrm{~cm}$ in $\mathrm{x}$ and $-3.175 \mathrm{~cm}$ in $\mathrm{z}$. The solenoids are moved from their ideal positions by $+3 / 16$ " and $-3 / 16$ " respectively. These were done with an pointlike, ideally placed beam and the $(\mathrm{mm})$, low dispersion solenoid tune. Table 7.2.2 shows the acceptances for these cases.

Table 7.4: Magnet offsets (units $=\mathrm{cm}$ ). Acceptance is for $2^{\prime \prime}$ plug.

\begin{tabular}{|c|c|c|c|c||c|c|}
\hline Solenoid 1 Y & Solenoid 2 Y & Dipole X & Dipole Z & Tune & Acceptance & Figure \\
\hline+0.476 & -0.476 & -1.1906 & -3.175 & MM & 0.16 & 7.17 \\
\hline
\end{tabular}



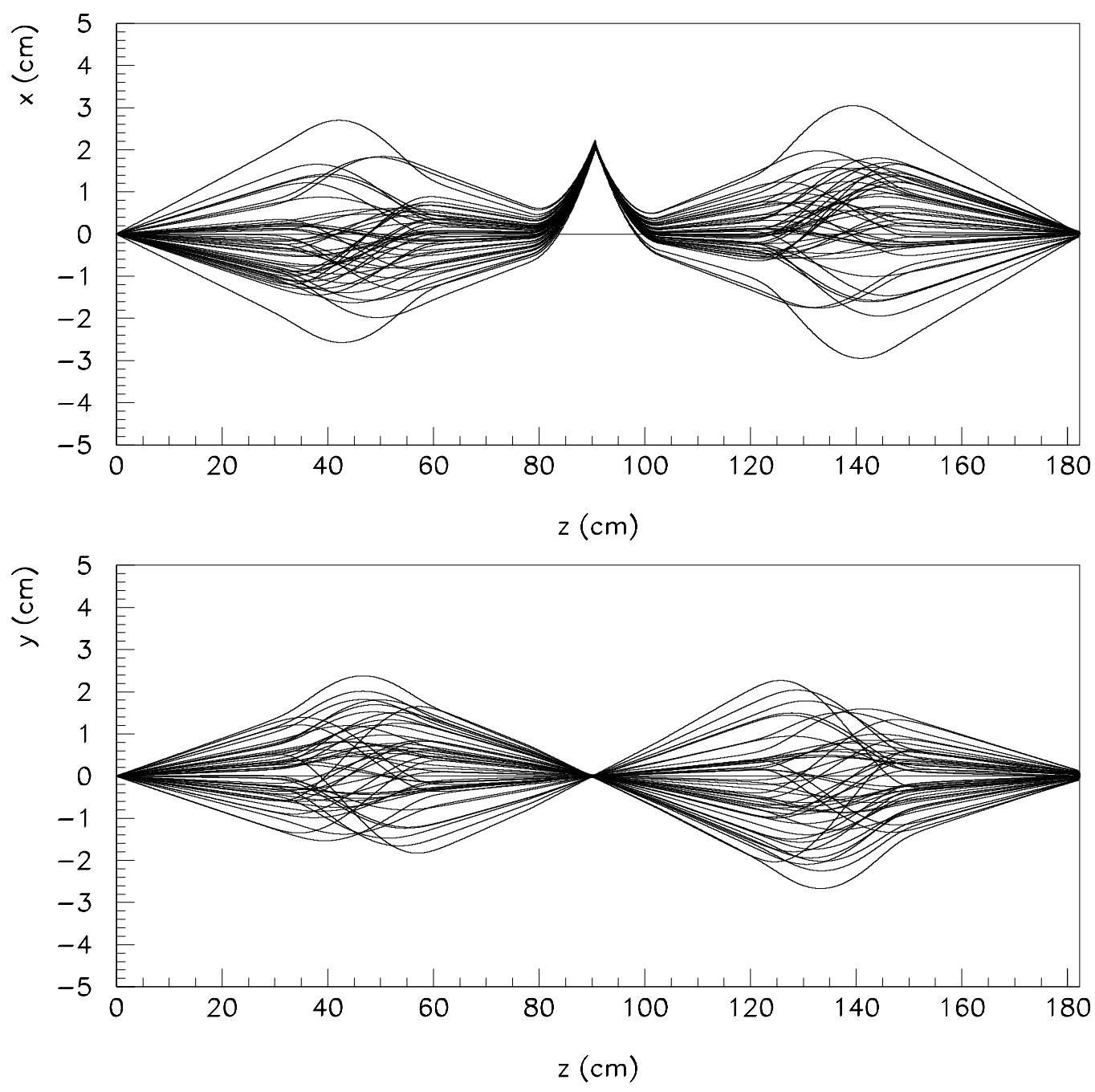

Figure 7.10: Ideal tracking using low dispersion, plus-minus (pm) solenoid tune for $e^{+}$corresponding to a $3 \mathrm{GeV} / \mathrm{c}$ momentum $\bar{p}$ beam. 

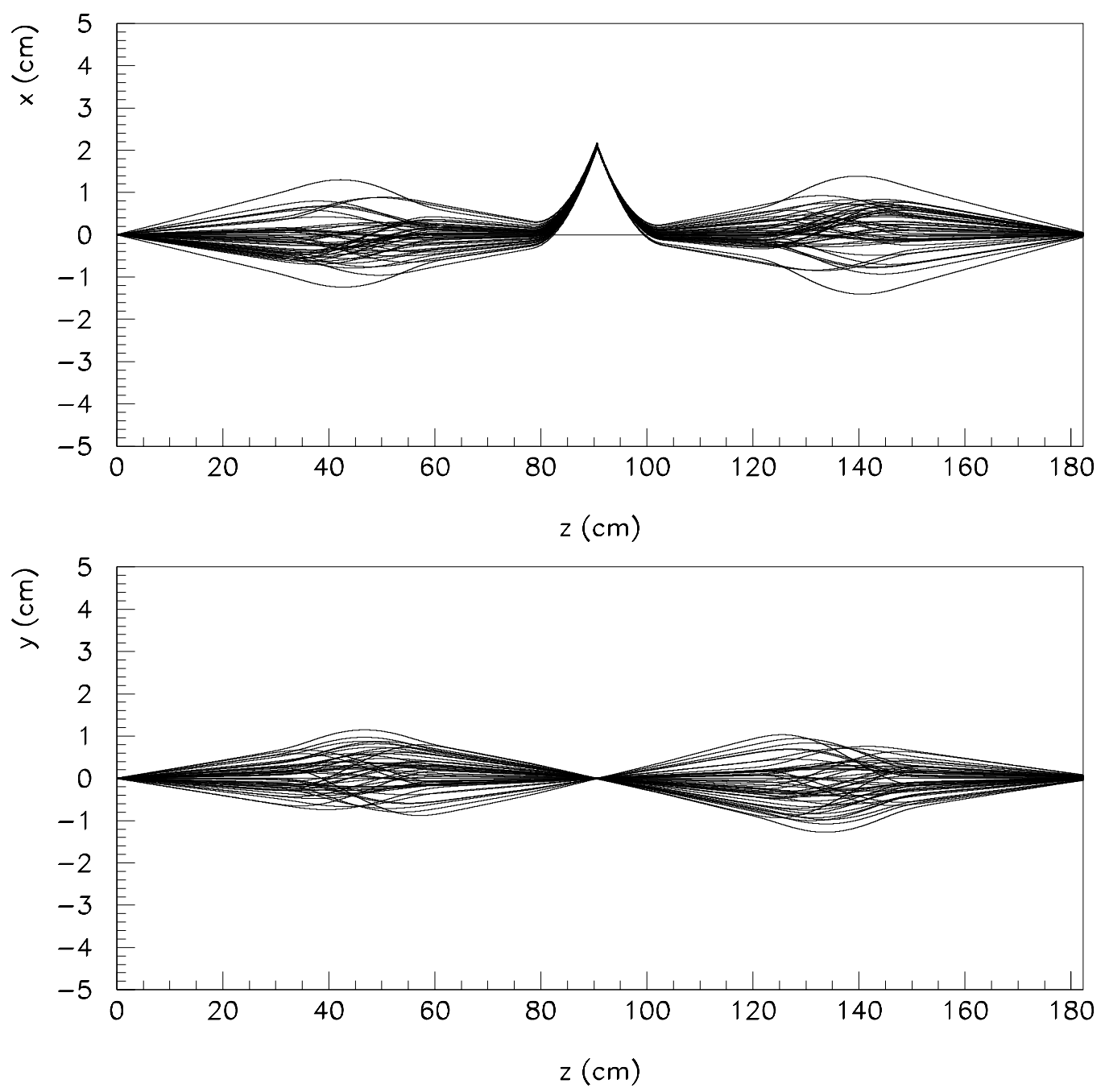

Figure 7.11: Ideal tracking using low dispersion, pm solenoid tune for $e^{+}$corresponding to a $6 \mathrm{GeV} / \mathrm{c}$ momentum $\bar{p}$ beam. 

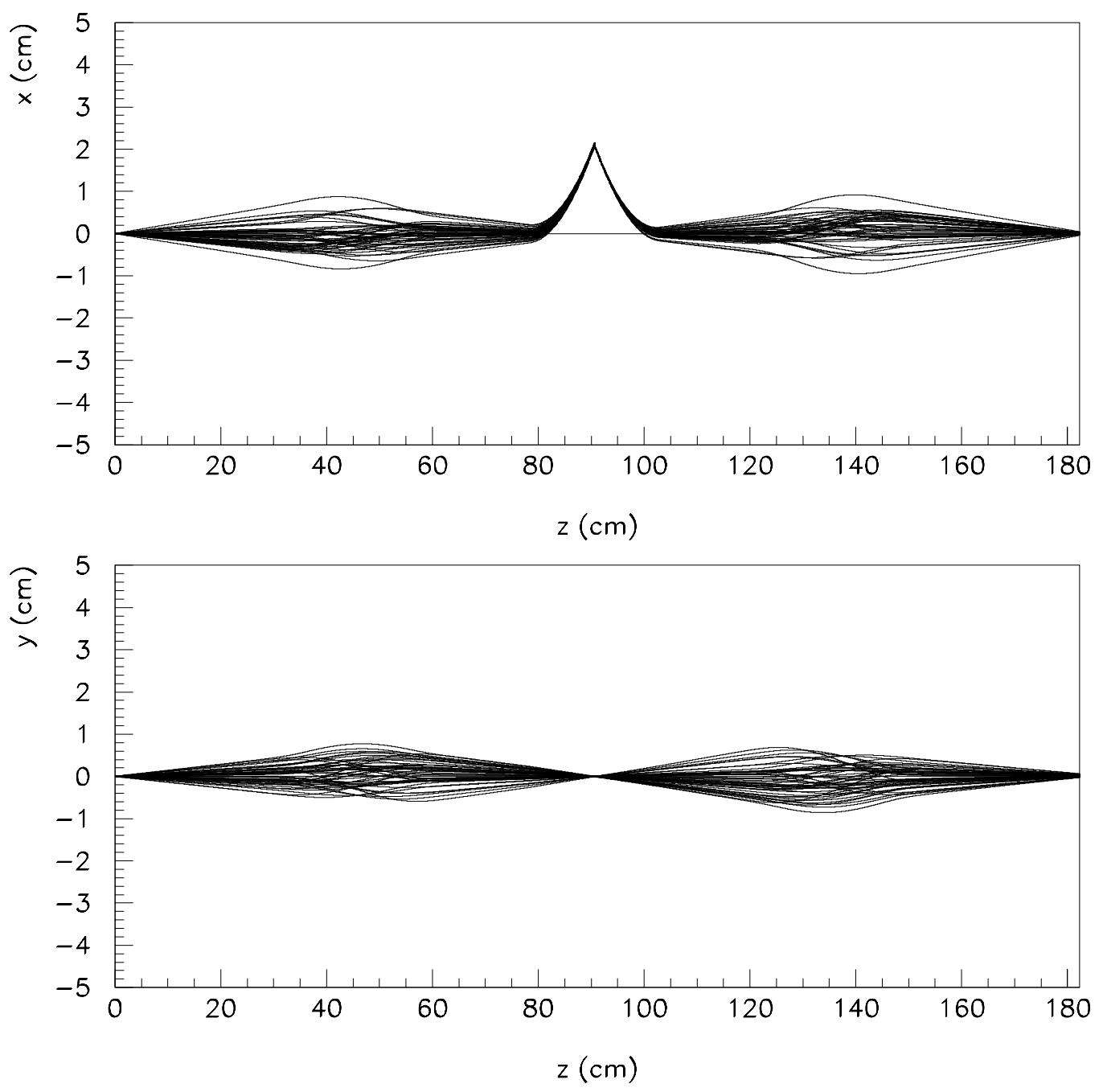

Figure 7.12: Ideal tracking using low dispersion, pm solenoid tune for $e^{+}$corresponding to a $8.85 \mathrm{GeV} / \mathrm{c}$ momentum $\bar{p}$ beam. 

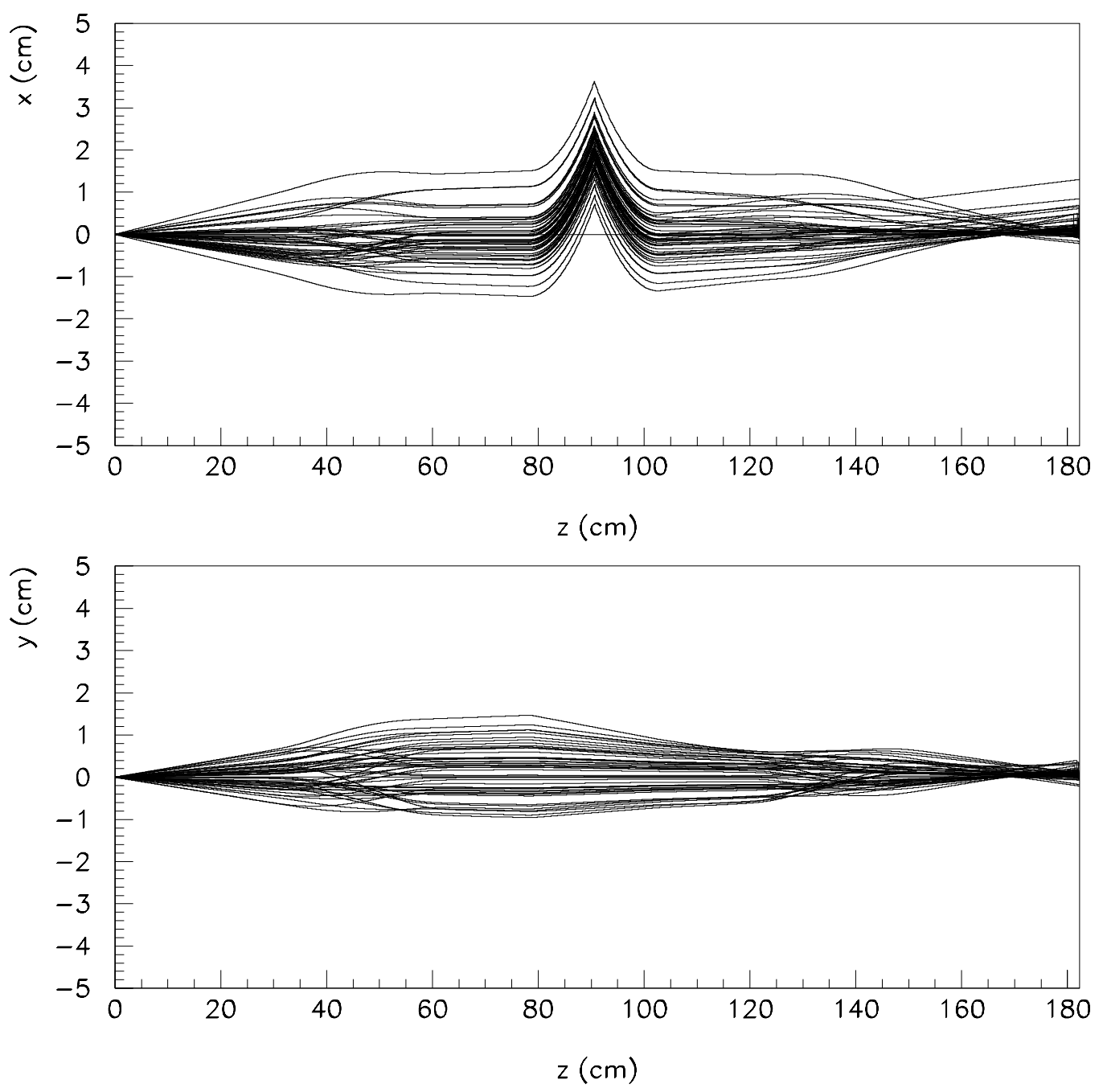

Figure 7.13: Ideal tracking using high dispersion, pm solenoid tune for $e^{+}$corresponding to a $6 \mathrm{GeV} / \mathrm{c}$ momentum $\bar{p}$ beam. 

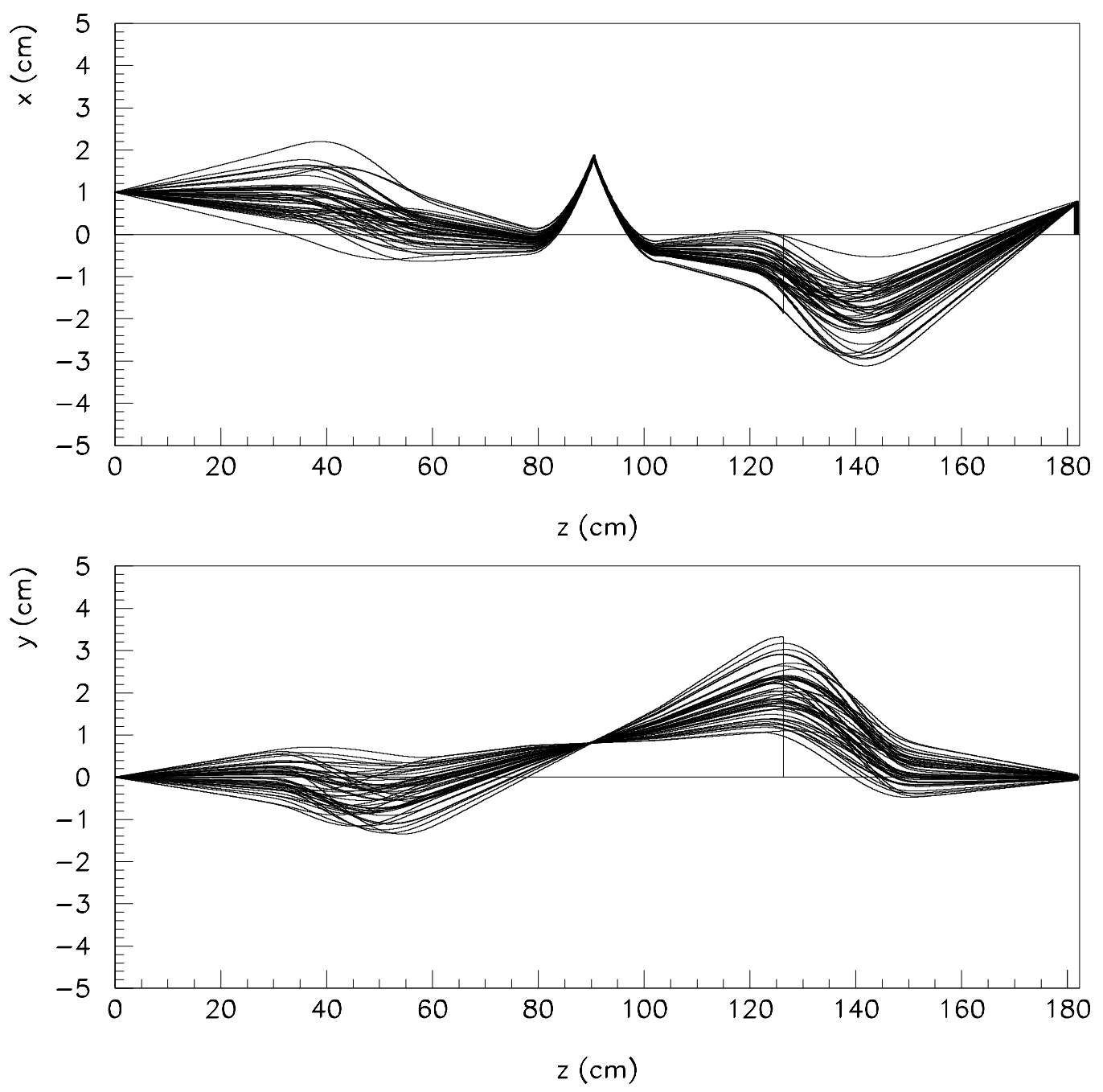

Figure 7.14: Ideal tracking with source offset of $1 \mathrm{~cm}$ in $\mathrm{x}$ for $e^{+}$corresponding to a $6 \mathrm{GeV} / \mathrm{c}$ momentum $\bar{p}$ beam. 

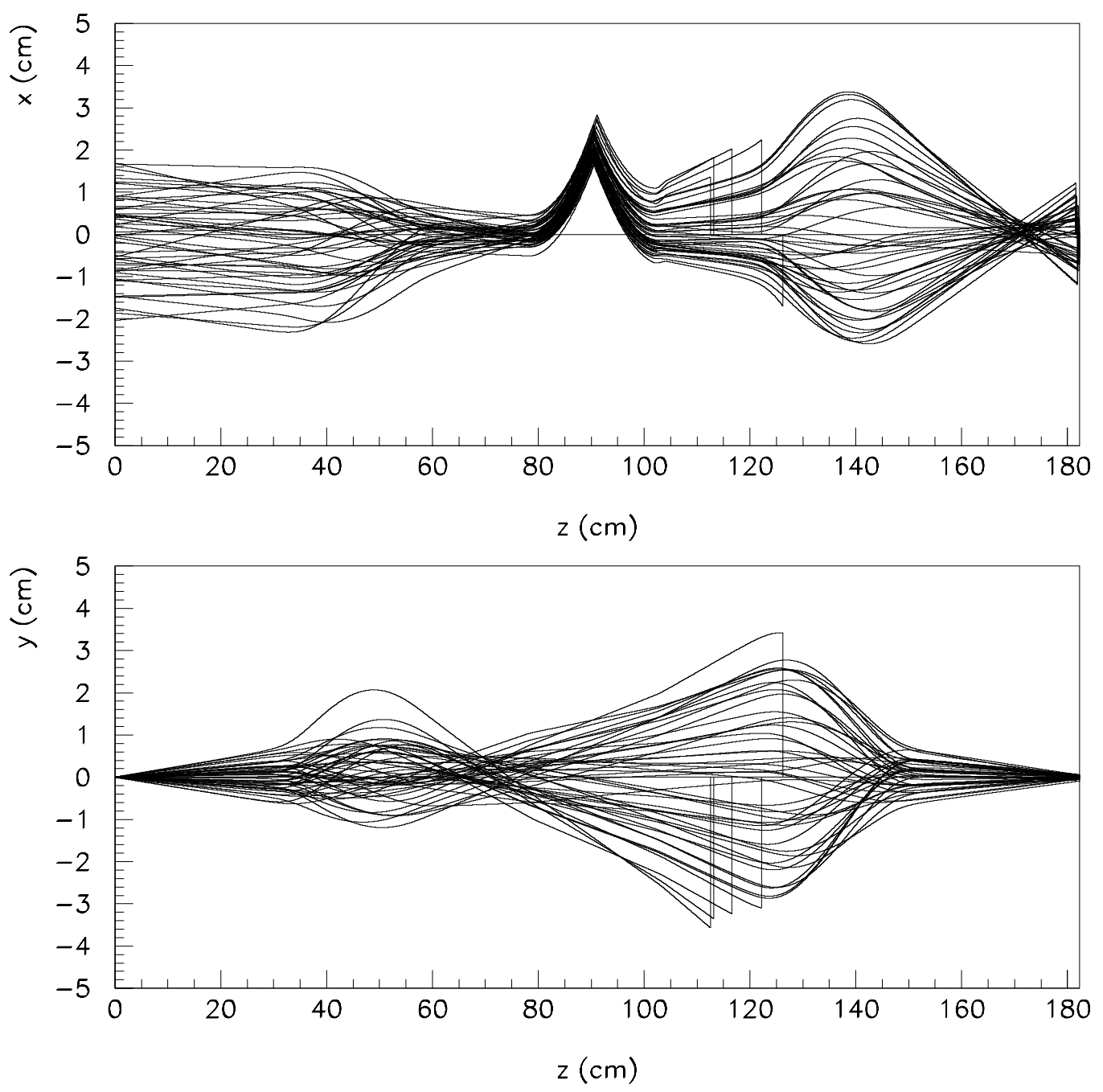

Figure 7.15: Ideal tracking with source spread of $1 \mathrm{~cm}$ in $\mathrm{x}$ for $e^{+}$corresponding to a $6 \mathrm{GeV} / \mathrm{c}$ momentum $\bar{p}$ beam. 

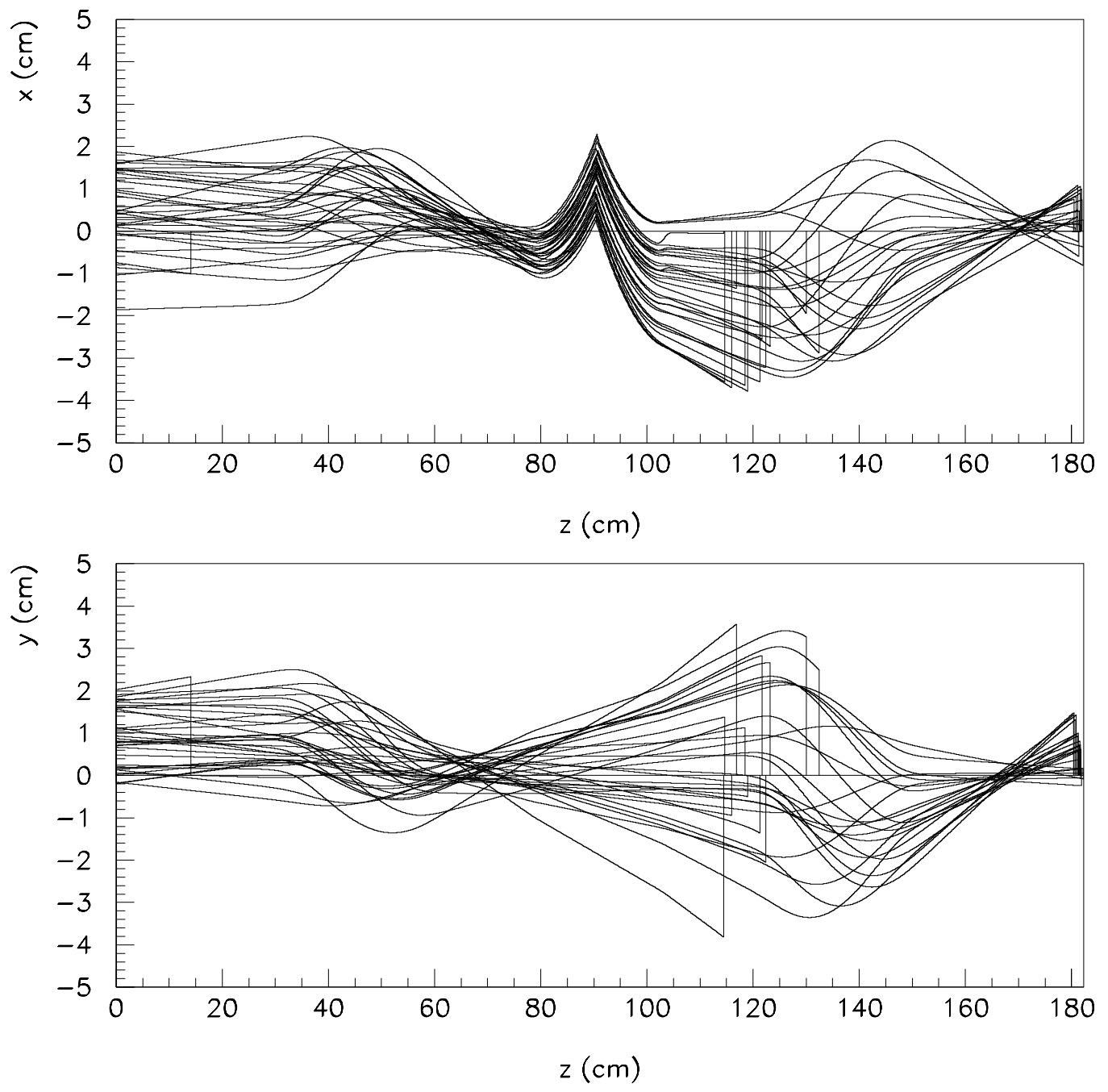

Figure 7.16: Ideal tracking with source offset of $1 \mathrm{~cm}$ in both $\mathrm{x}$ and $\mathrm{y}$ and a source spread of $1 \mathrm{~cm}$ in both $\mathrm{x}$ and $\mathrm{y}$ for $e^{+}$corresponding to a $6 \mathrm{GeV} / \mathrm{c}$ momentum $\bar{p}$ beam. 

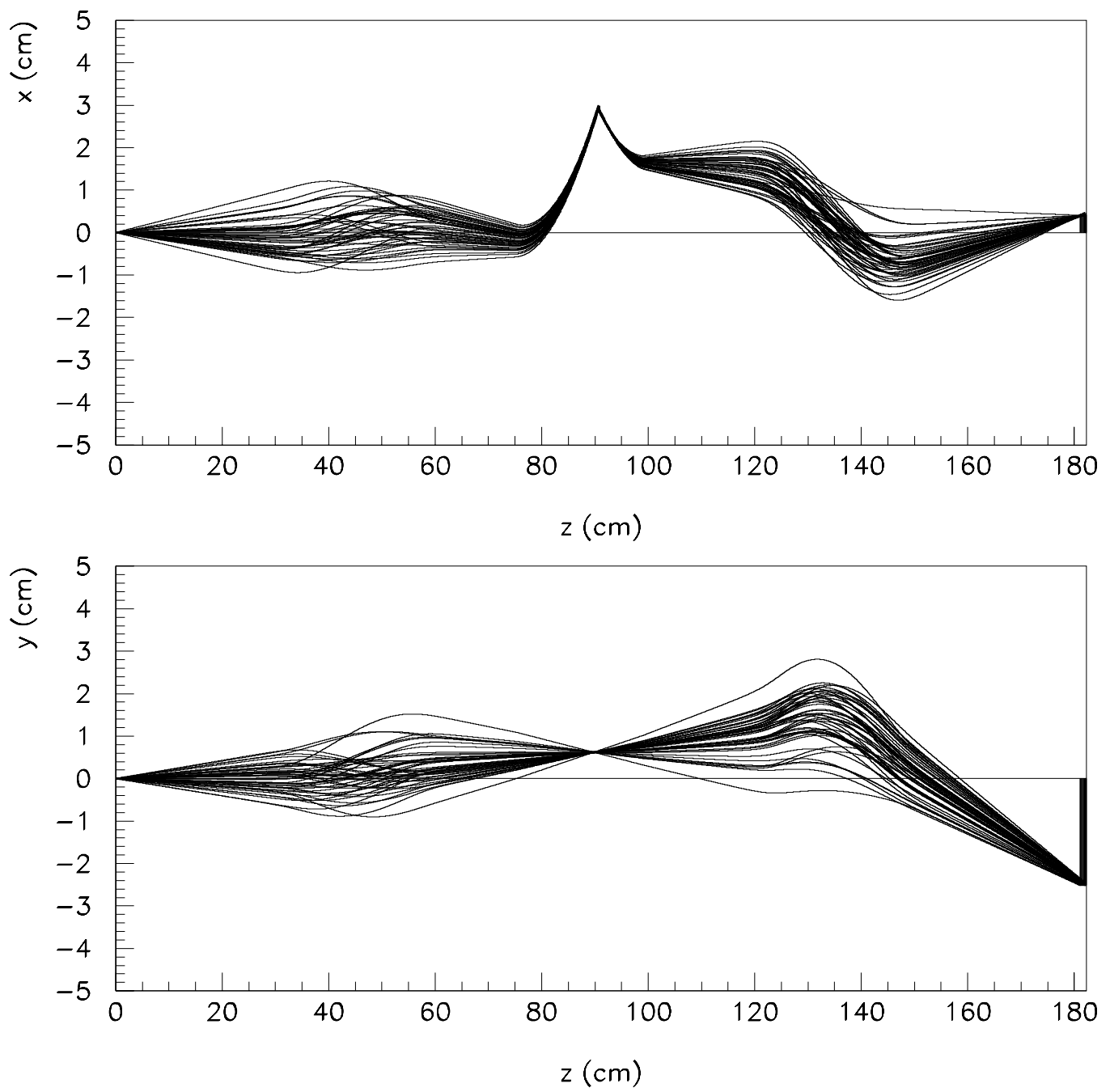

Figure 7.17: Tracking with solenoid \#1 moved $+0.476 \mathrm{~cm}$ in y and solenoid \#2 moved $-0.476 \mathrm{~cm}$ in $\mathrm{y}$ and the dipole moved $-1.1906 \mathrm{~cm}$ in $\mathrm{x}$ and $-3.175 \mathrm{~cm}$ in $\mathrm{z}$ (using the coordinate system before the $40^{\circ}$ bend. This is for $e^{+}$corresponding to a $6 \mathrm{GeV} / \mathrm{c}$ momentum $\bar{p}$ beam. (mm tune) 
For real run-by-run efficiencies, the data supplied by ACNET (momentum, beam centroid position, emittance) are used to define the starting distribution of positron kinematic variables for each unique (run number-momentum) combination. After distributions are constructed, cuts are made on the foil size (1" diameter until run 51 when it was increased to 1.75 " diameter) as well as the apertures of the beam pipe and the plastic scintillator size (1" diameter until run 51,2" diameter afterwards). Figures 7.18 , and 7.19 show, respectively, versus the run-momentum combination, the geometrical acceptances for an antihydrogen striking the foil target, and the positron to make it through the $e^{+}$spectrometer. Obviously, the beam steering affects the $e^{+}$spectrometer acceptance. The spectrometer is assumed to be in its ideal configuration with the plus-minus solenoid tune (should yield zero net image rotation), and no magnet position offsets. The net acceptance of the spectrometer is just the ratio of the total to the beam steering acceptance. Steering the $\bar{H}^{o}$ onto the foil is independent of getting the subsequent $e^{+}$through the spectrometer. Each separate acceptance function has a similar dependence on beam parameters (i.e. the deviation of beam parameters from an ideal beam). The spectrometer acceptance being almost equal to $100 \%$ early in the run is simply saying that anything that can get through a 1 inch hole is going to be far from the apertures in the spectrometer. Later on, switching to the 1.75 " diameter foil allows $e^{+}$s to start with transverse positions further from the center of the spectrometer beamline. In addition to being closer to the apertures, they can also be affected by slightly nonlinear sections of the magnetic fields. The latter is expected to be a small effect, though. 


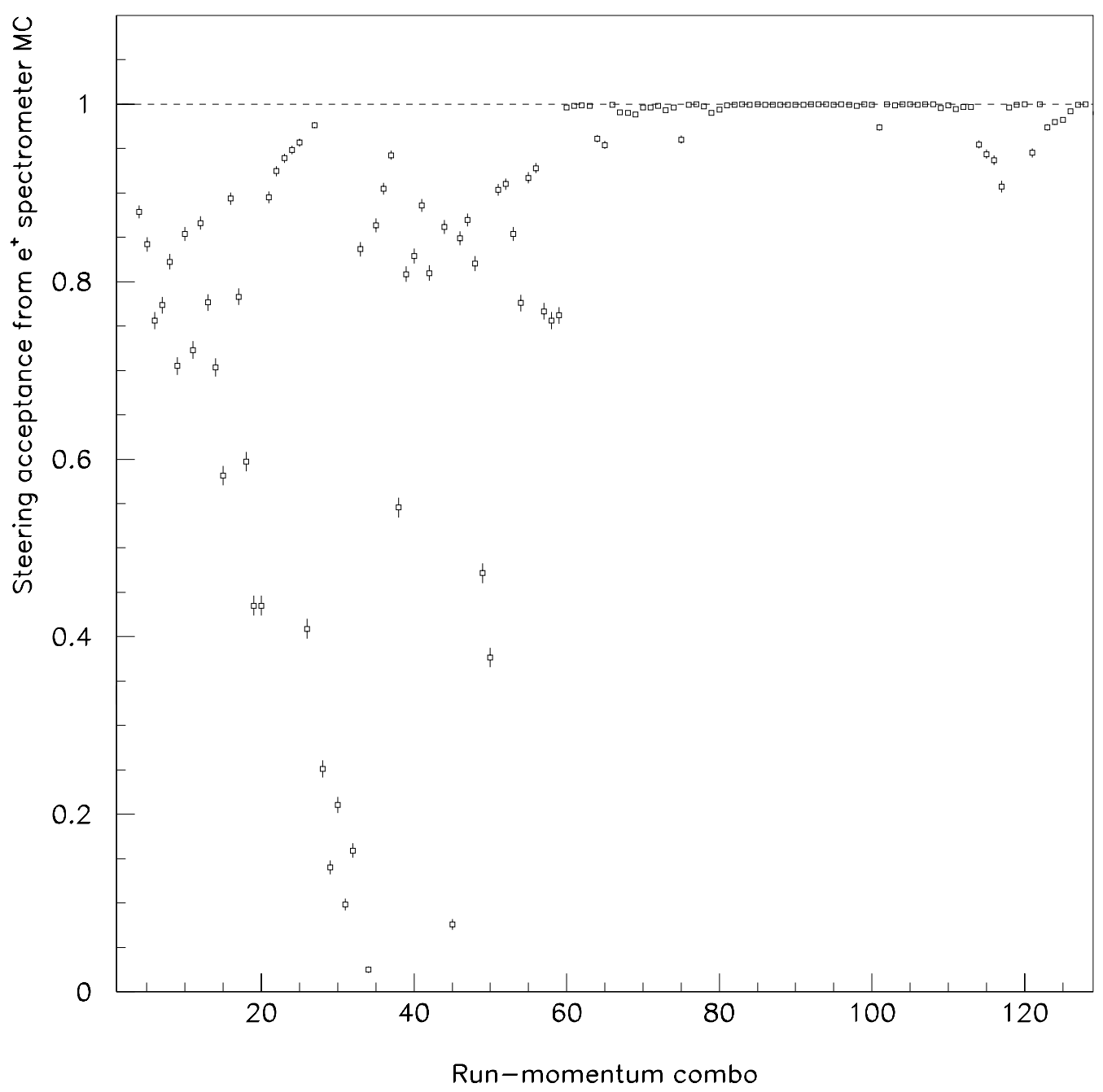

Figure 7.18: Geometrical acceptance of antihydrogens for hitting the foil target plotted versus run-momentum combination. 


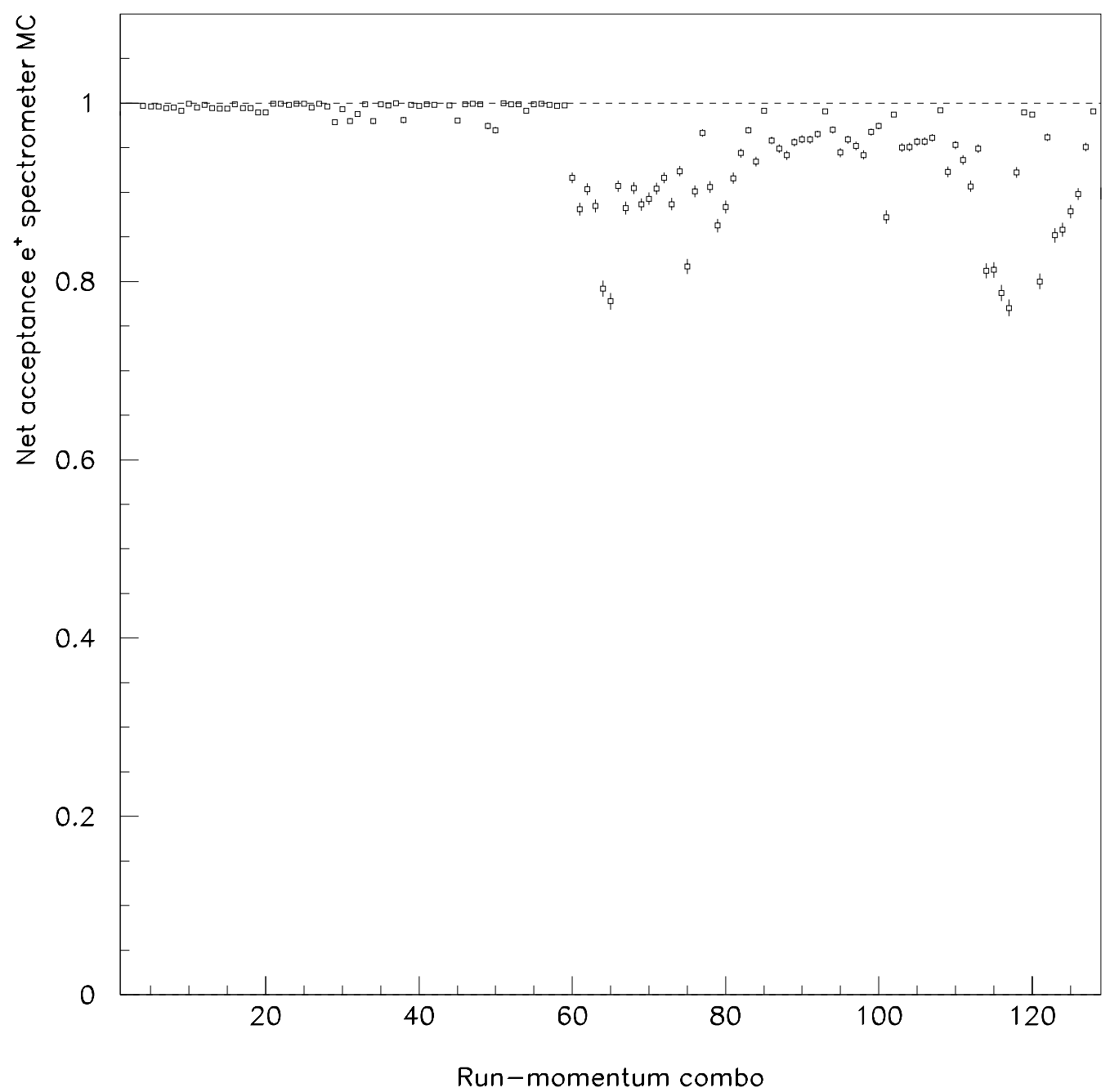

Figure 7.19: Geometrical acceptance for the positron to make it through the $e^{+}$ spectrometer to intercept the scintillator. This is plotted versus run-momentum combination. 


\subsection{Simulation of antiproton momentum reconstruc- tion}

Figure 7.20 shows the fractional momentum distribution resulting from a foilout Monte Carlo - generated set of events which are reconstructed by the offline code. Figure 7.21 shows the fractional momentum distribution extracted from the data for all candidate events having tracks, high momentum track events with no positron, and elastic scattering data peaked near $\mathrm{P} / \mathrm{Pbeam}=0.975$ which was used to bound the momentum spread. 


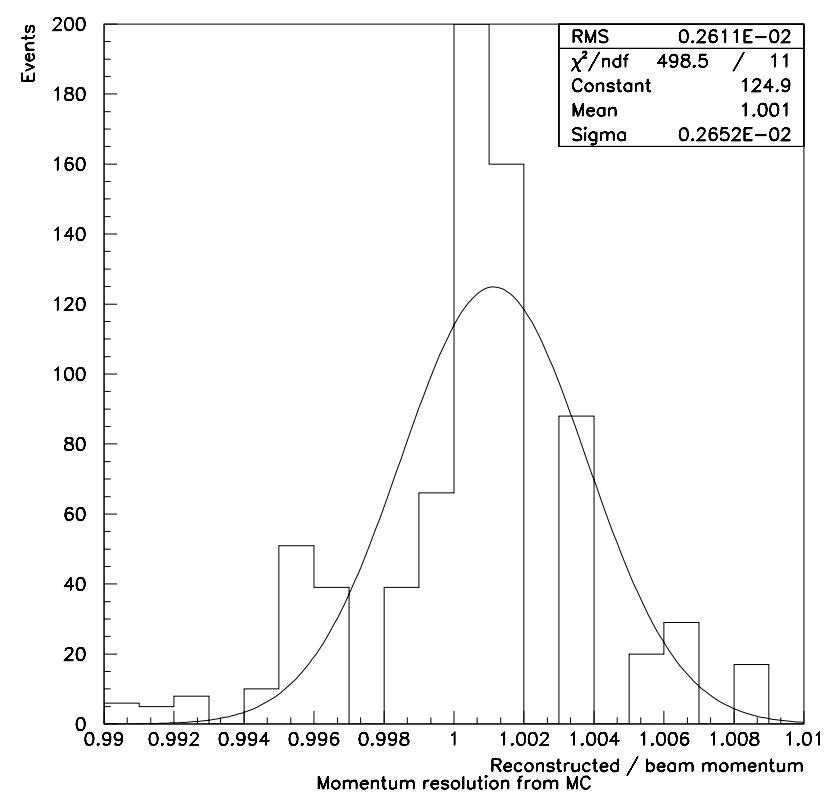

Figure 7.20: Momentum resolution from Monte Carlo.
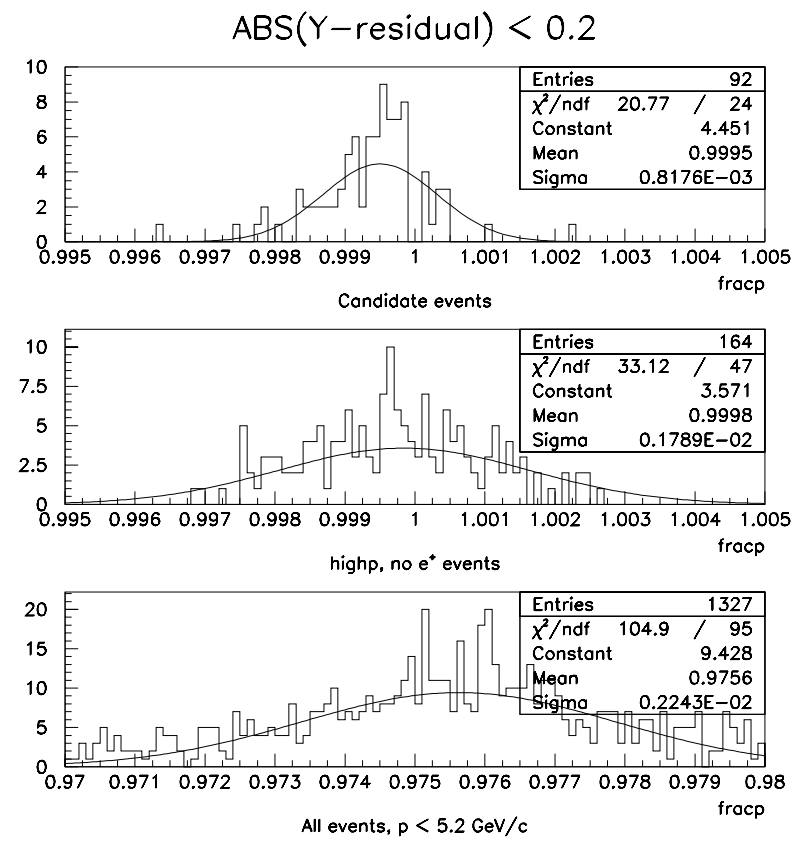

Figure 7.21: Momentum resolution from data sample containing only candidate events. 


\subsection{Efficiencies}

There were also a small number of events under the $T F 1 \otimes e^{+}$trigger which had a reconstructed track but missed TF2. These are events whose PWC \#3 space points and angles point to TF2, but which don't actually trigger the counter. Figure 7.22 shows the distributions of horizontal position in PWC \#3 for all events with one track and $\left|Y_{\text {res }}\right|<0.15$ inch, candidate events, and high momentum, non-candidate events. The TF2 counter is close to PWC \#3 so the edge of TF2 can be seen as a drop in the number of events in the top plot. A beam momentum, centered track with a small angle, such as expected for antihydrogen events should be well away from the edge. The candidate events are contained within a gaussian distribution; $95 \%$ of its area is on the viewable side of TF2's edge. The distribution of the non-candidate event positions is wider and so a significant loss in geometric acceptance is possible.

\subsubsection{Trigger Efficiencies}

The trigger efficiencies for $T F 1 \otimes T F 2$ and $T F 1 \otimes e^{+}$were hard to gauge because there was no high statistics baseline process to use. To some extent, the antihydrogen events were used as a sample since any two of the three triggers used are independent. They were also guaranteed, as seen in chapter 6 , to have the straightest, best centered tracks so geometric cuts can be ignored. Unfortunately, the low statistics prevent a run-by-run evaluation. They can only serve as an overall confirmation of another efficiency estimate. When TF2 became inefficient towards later runs, triple coincidences were unaffected because the $e^{+}$arm is independent and the tracking shows a good event even without that counter. The number of triple coincidences lacking a TF2 part of the trigger verified the loss of efficiency seen in the raw scaler rates. Another way of getting the first trigger's efficiency is to use the TF1 only 

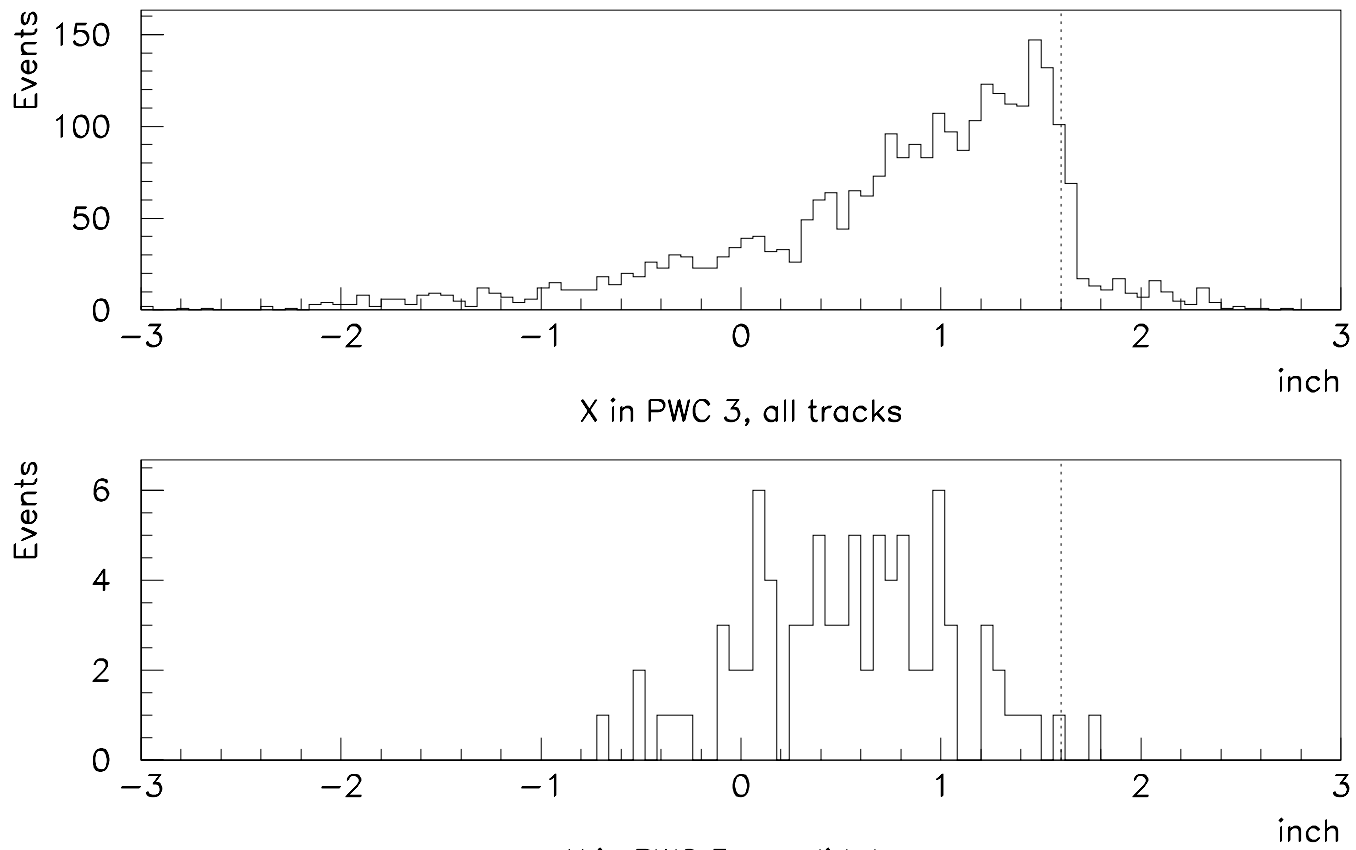

$X$ in PWC 3, candidates

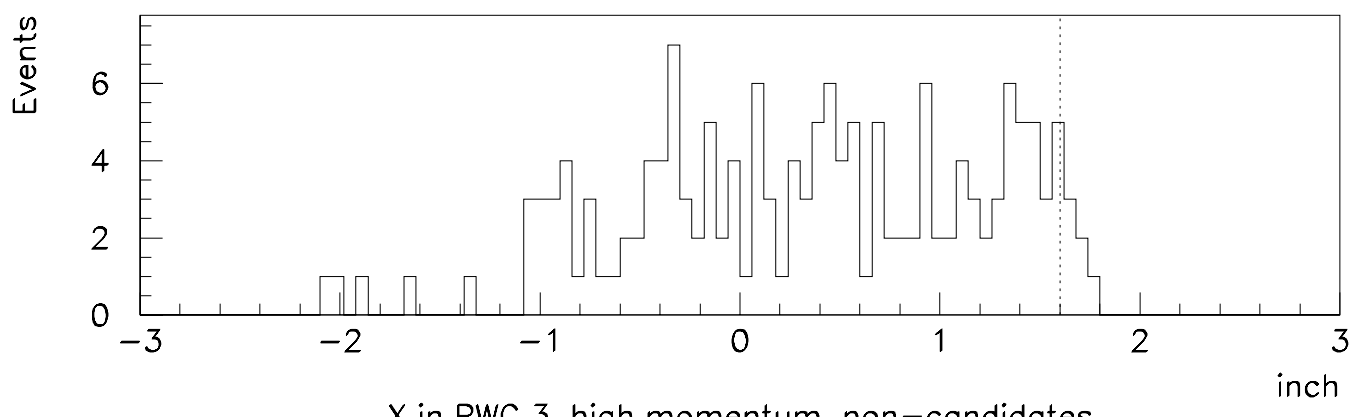

Figure 7.22: Horizontal position distribution in PWC \#3 for all tracks with $\left|Y_{\text {res }}\right|<$ 0.15 in, candidate events, and high momentum, non-candidate events. In the top plot, the right edge of the TF2, 4" $\times 4$ " area, counter is evident as a drop in number of events. 
(prescaled) data to count good tracks (which were mostly at $97.5 \%$ of beam momentum) which missed TF2. This was the most important trigger since it guaranteed a track. A way of looking for inefficiencies in the $e^{+}$counter's response is to compare the $e^{+}$pulse height spectrum of TF1 prescaled data with that of events containing $T F 1 \otimes e^{+}$in the trigger. Figure 7.23 shows that the TF1 prescaled data had no pulse heights larger than pedestal. It is possible that a positron can pass completely through the $e^{+}$counter but the probability is very small. One triple coincidence event was seen which suggests this as there is minimal energy in the $\mathrm{NaI}$ halves as well as no energy in the $e^{+}$counter. The ratio of scaler values for the separate TF1 phototubes to the scaler values for the coincidence is shown in figure 7.24. The ratios are constant after run 5 except between runs 70 and 80 . This is a strong indication that neither phototube was failing over time. The TF1 threshold was set so low, $(<<1 / 10 \mathrm{MIP})$, after run 20, that losing events because of the threshold is inconceivable. In addition, only a handful of events were seen in the data satisfying only the $T F 2 \otimes e^{+}$trigger and no other. None of these had any $e^{+}$counter pulse height or TDC hit so they were judged as electronic noise. None of these had any hint of a track. If an antiproton from antihydrogen passed through TF1 without depositing energy, it would leave a hit in the TF2 and $e^{+}$counters.

In addition, the triggers were formed from loose coincidence within a $75 \mathrm{~ns}$ overlap. There was no chance that part of the antihydrogen signals were out of time. The loose coincidence provided a way for random coincidences to be recorded by the $T F 1 \otimes e^{+}$trigger, especially. These were easily filtered out.

The TF2 trigger efficiency is shown in figure 7.25. It shows a dramatic decrease towards the later runs due to one of the phototube pulse heights becoming much smaller. This efficiency is calculated by assuming one of the two phototubes signals used in the coincidence was not performing towards the end of the running. The 

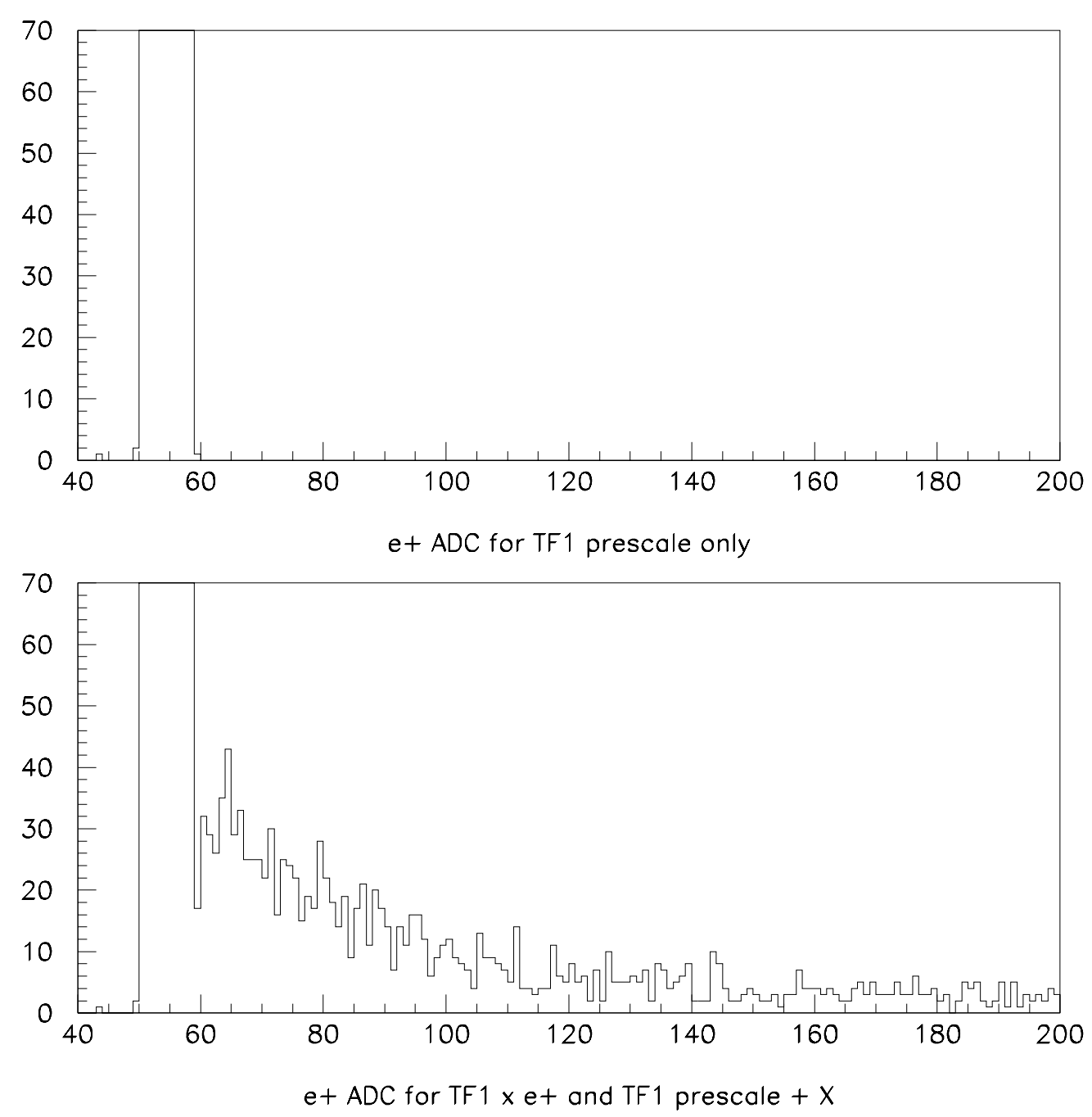

Figure 7.23: Comparison of $e^{+}$pulse height for events only satsifying the TF1 only (prescaled) trigger and those which satisfy this trigger and the $T F 1 \otimes e^{+}$as well. 
Rates from scalers averaged over each run
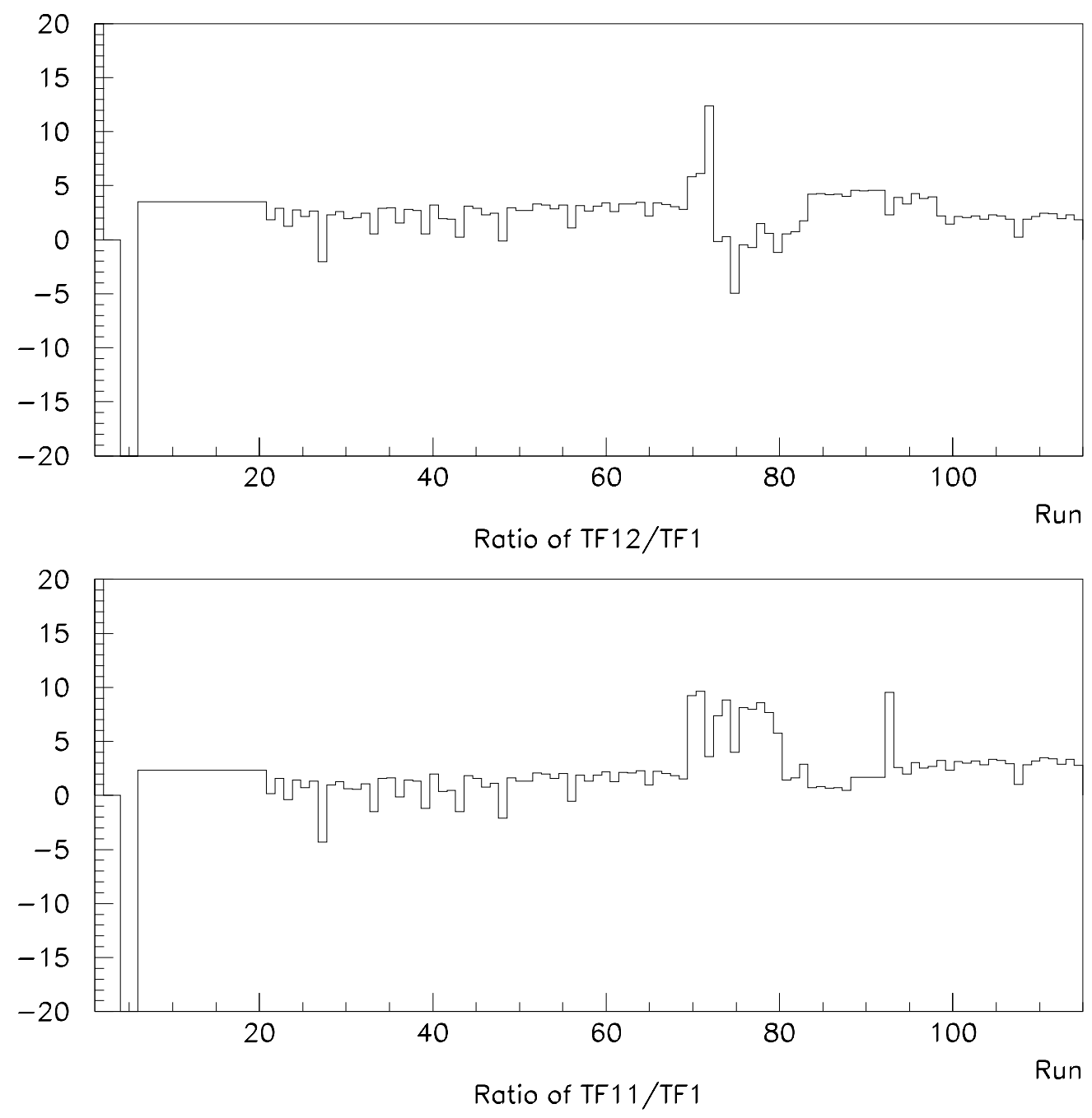

Figure 7.24: Ratios of TF1 scalers. 
efficiency shown is the ratio of scaler rates of the tube assumed to be working correctly divided by the coincidence rate. The ratio is then normalized to be less than or equal to one. The threshold was relatively high for these counters so that the rates are expected to correspond reasonably well with the rate for a true sample from minimum ionizing particles. High momentum tracks will always leave about $2.6 \mathrm{MeV}$ unless they are close to the edge of the counter.

\subsubsection{Tracking Efficiency}

Acceptance $\times$ Efficiency values for the chambers were calculated by counting the number of tracks with all but one plane on divided by the number of tracks formed with all possible planes on (having $\geq 1$ hit). Events with only one track were used. They are presented separately for each run in figure 7.26. Some planes were not instrumented for a set of runs. In addition, some planes show an obvious constant efficiency less than 1.0 since their geometrical coverage is less than the other planes for the same chamber. So space points are made from 2 of the 3 active planes $75 \%$ of the time instead of three planes (for PWC \#1, for example) in the later data. Keep in mind that this averages over the transverse position distribution. Since antihydrogens are almost all in the center of the PWCs, they are only affected by the actual efficiency of the chambers not the geometrical acceptance.

The three-way coincidences are the best signature of antihydrogen, which also means they have straight and centered trajectories. The wire chamber efficiencies can also be calculated by counting the number of three-ways which miss a certain plane. The results using a sample of 61 events with tracks from run 14 on are shown in table 7.4.2. The requirements for forming the tracking efficiency are the same. At least 


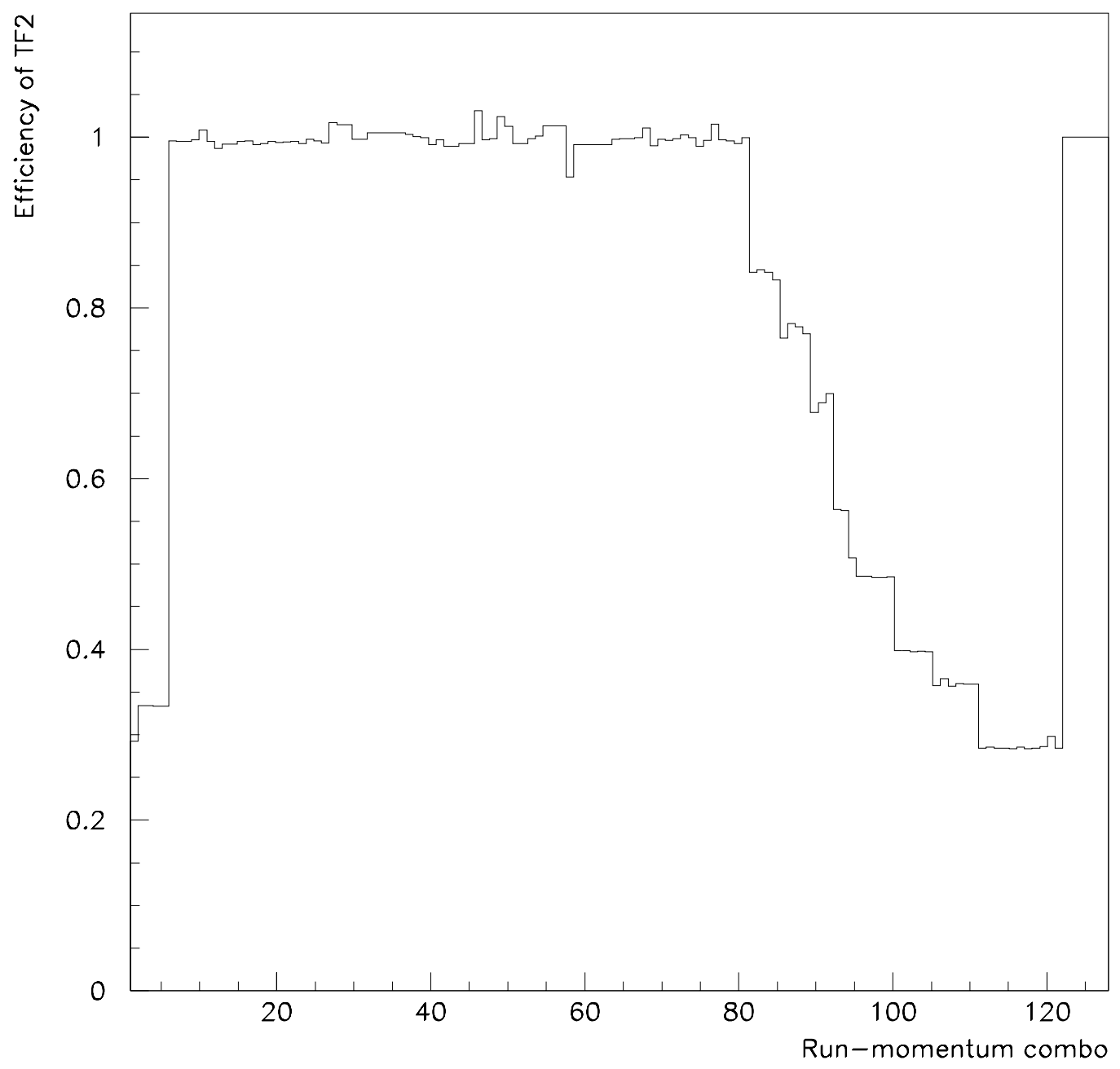

Figure 7.25: Efficiencies for TF2 part of trigger. The abscissa is not E862 run number but a combination of run number and momentum since some runs had more than one momentum. 
Table 7.5: Wire chamber and tracking efficiencies from three-way coincidences.

\begin{tabular}{|c|c|}
\hline Plane & Efficiency \\
\hline \multicolumn{2}{|c|}{ PWC \#1 } \\
\hline 1 & 0.85 \\
2 & 0.93 \\
3 & 0.97 \\
\hline \multicolumn{2}{|c|}{ PWC \#2 } \\
\hline 1 & 0.93 \\
2 & 0.97 \\
\hline \multicolumn{2}{|c|}{ PWC \#3 } \\
\hline 1 & 0.92 \\
2 & 0.87 \\
3 & 0.92 \\
\hline Tracking Efficiency \\
\multicolumn{2}{|c|}{0.992} \\
\hline
\end{tabular}

two hits are needed to form a space point. A track is formed if at least two of three chambers have a space point. No assumptions are made on the quality of the track.

\subsubsection{Efficiencies Summary}

The data acquisition system was not fully efficient since it had to handle file transfers and CAMAC polling. For each run, the visual scalers for total triggers and triggers taken by the DAQ were compared and the ratio recorded as the live time fraction. The average of the live time fraction measurements was 0.9699. Scaler counts during beam loss periods were not considered.

The E835 integrated luminosities were multiplied by the E835 data acquisition system's live time fraction (usually $>0.95$ ) before being written to disk. These live time fractions were averaged over the E835 run numbers corresponding to each E862 run number-momentum combination weighted by the integrated luminosity. These numbers are then divided out of the total acceptance $\times$ efficiency before making the correction to the data. 

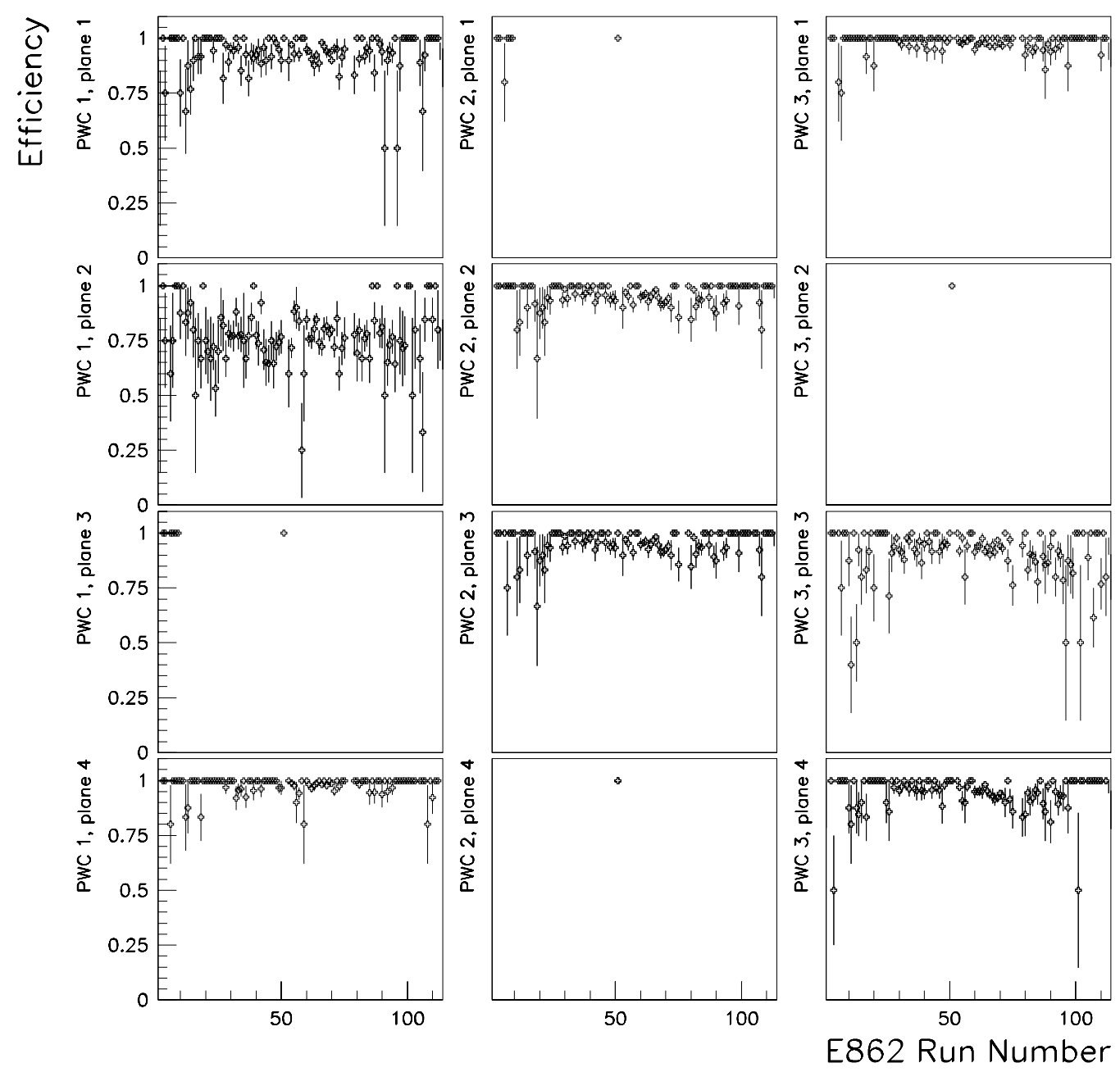

Figure 7.26: Number of tracks missing a hit in only one plane divided by the number of tracks total. Certain planes do not have data since they were not instrumented for those runs. 
Table 7.6: Final corrections to data.

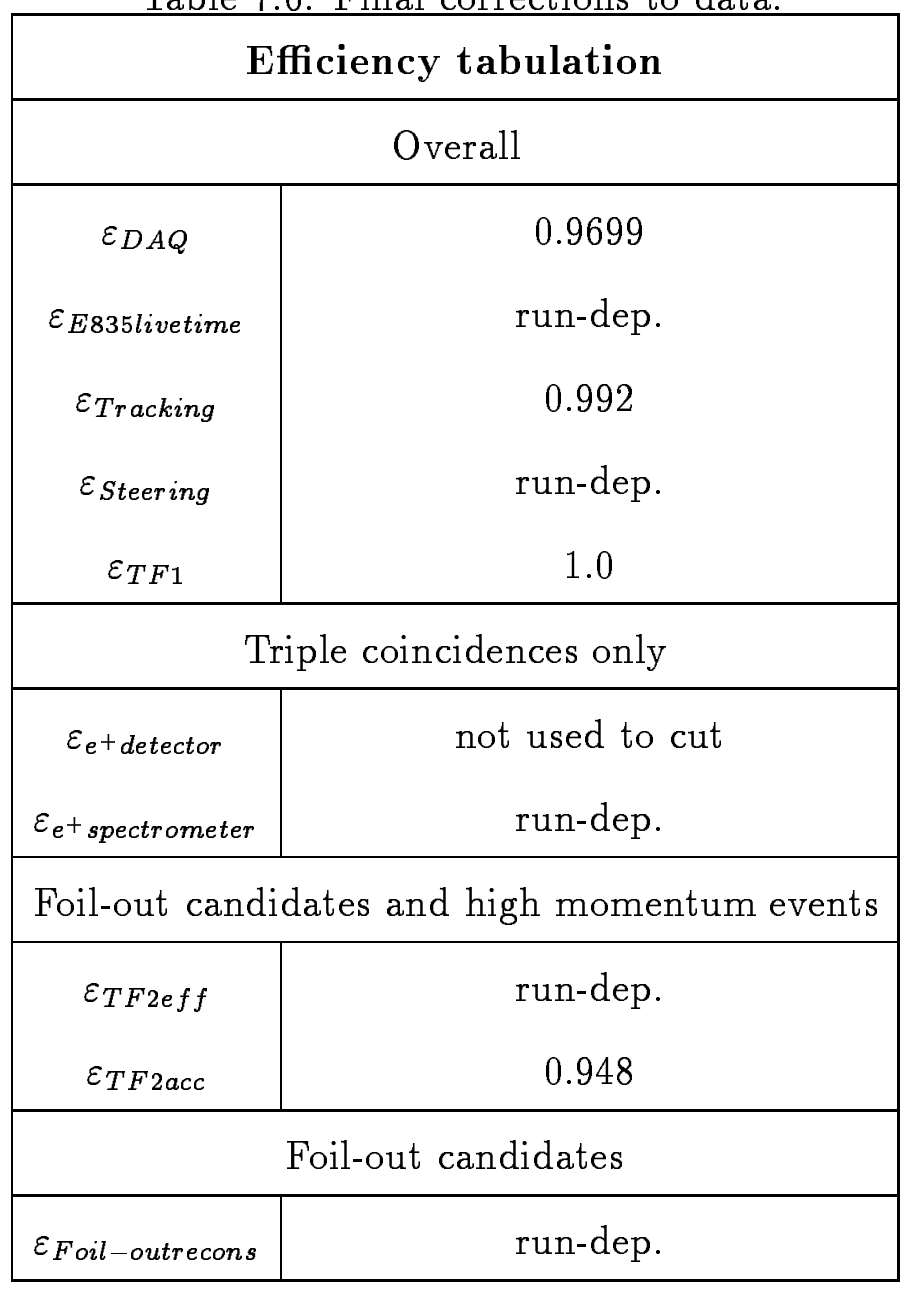


Figure 7.27 shows the total efficiency for foil-out events. The antiproton tracking efficiency and DAQ live time, are not included in figure 7.27.

Figure 7.28 shows the total correction vs run-momentum combination applied to the triple coincidence candidates.

$$
\varepsilon_{\text {Three-way }}=\left(\varepsilon_{\text {Steering }} * \varepsilon_{e^{+} \text {spectrometer }}\right) *\left(\varepsilon_{\text {Tracking }} * \varepsilon_{D A Q}\right)
$$

$$
\varepsilon_{\text {Foil-out }}=\left(\varepsilon_{\text {Steering }} * \varepsilon_{T F 2 a c c}\right) *\left(\varepsilon_{T F 2 e f f} * \varepsilon_{\text {Foil-outrecons }} * \varepsilon_{\text {Tracking }} * \varepsilon_{D A Q}\right)
$$




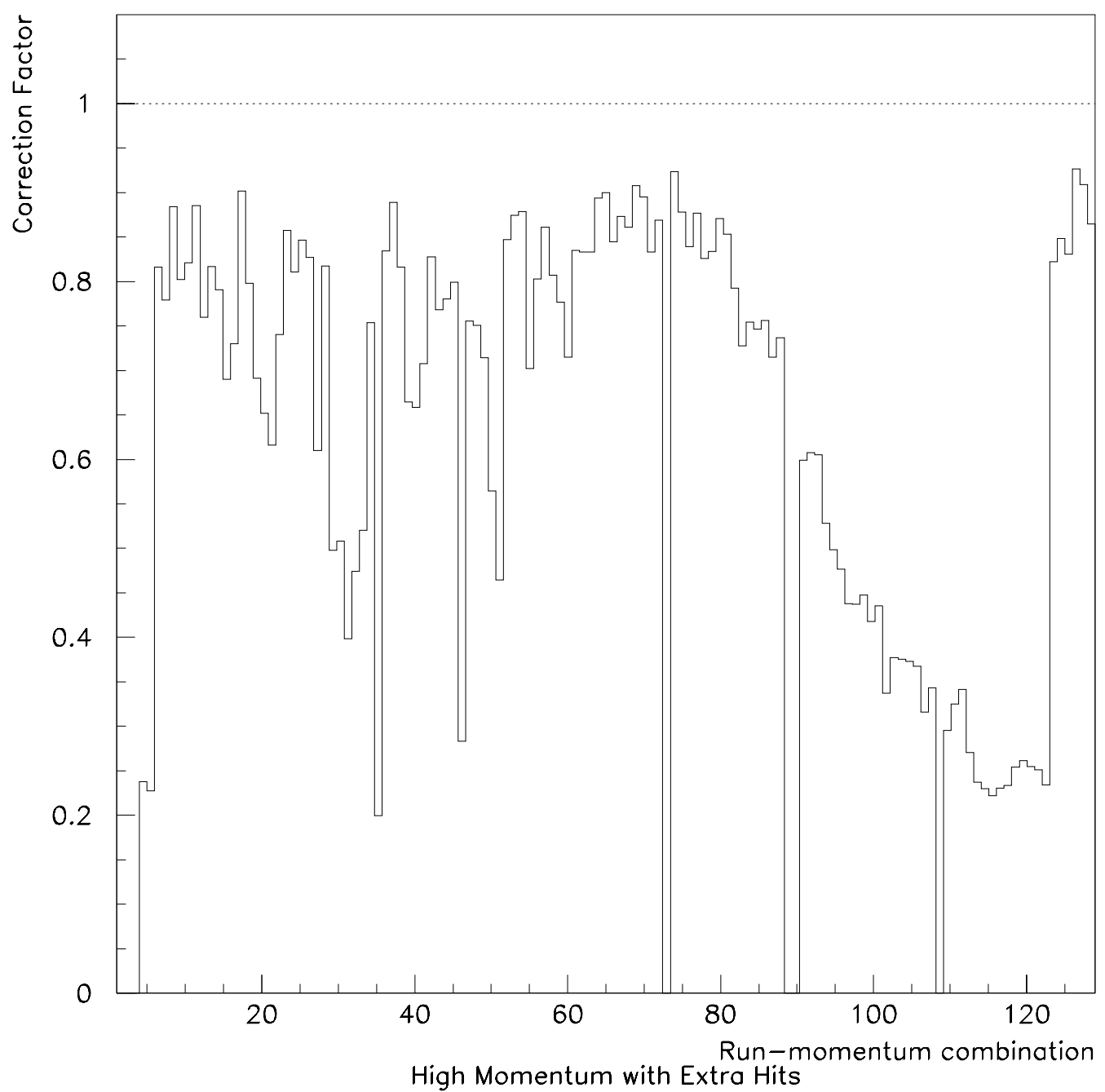

Figure 7.27: Total efficiencies for detecting a foil-out type antihydrogen candidate event. 


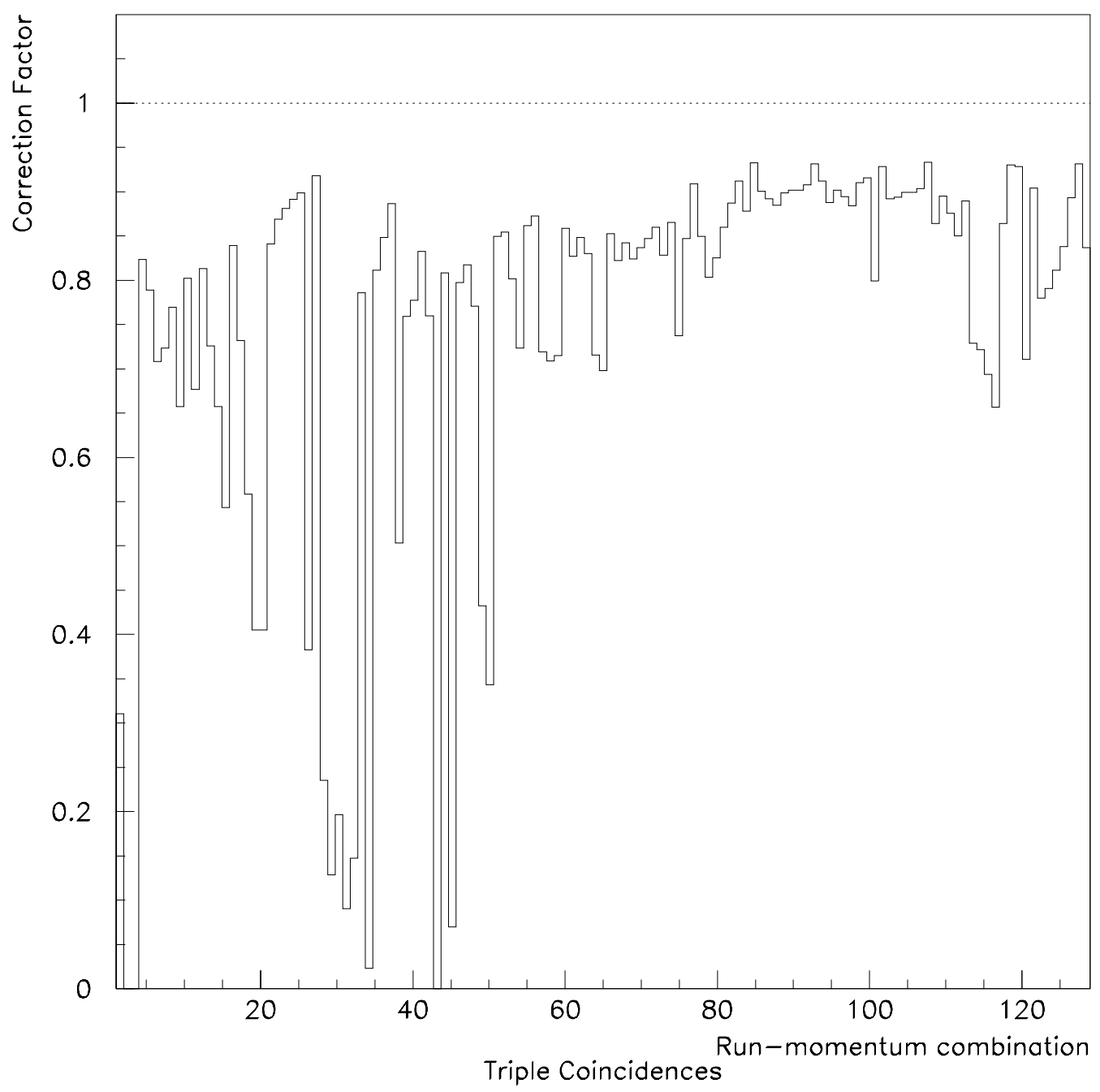

Figure 7.28: Total efficiencies for detecting a triple coincidence type antihydrogen candidate event. 


\section{Chapter 8}

\section{Results and Conclusions}

First, a case is made that E862 definitely observed antihydrogen atoms. The events are tabulated for the entire running period. The cross section calculations are presented afterwards. The results are a good measurement of the cross section at about $6 \mathrm{GeV} / \mathrm{c}$ antiproton momentum and a not-so-good measurement of the energy dependence of the cross section using the entire range of data. A systematic error discussion and conclusions follow.

\subsection{Observation of Antihydrogen Events}

Experiment E862 originally intended only to detect antihydrogen atoms using the triple coincidence signature which, at least, includes the coincidence of the three fast scintillation counters described in chapter 5 . The foil target was removed periodically to prove that the triple coincidence signal was purely the result of a beam hitting the thin foil. No triple coincidences were seen when the foil was removed. This alone lends credence to the triple coincidence events being antihydrogen. Possible background reactions which could send a neutral particle down the beamline were discussed in section 6.5. The expected number of these which would be in the beamline, have an interaction at the foil location, and have by-products which would mock a triple coincidence signal are very small. Furthermore, to have the $e^{+}$pulse height 
agree with the expected value as well as having signals in the $\mathrm{NaI}$ indicating an annihilation, is even less likely unless the original particle is an antihydrogen atom.

An additional confirmation came when events were seen in the foil target-out data which had a high momentum track and had one extra hit in PWC \#1. It is true that an antiproton can knock an electron out of the vacuum window which could register in PWC \#1. We have calculated the number of electrons of this type coming from any momenta track observed and found it to account for no more than one event in all the data. If one makes a further cut demanding the track to be high momentum, the number of expected events becomes $<0.03$. The fact that we have observed these types of events and, furthermore, that the event rate is approximately the same is a great indication that they come from the same source, antihydrogen.

For example, if one puts forth the hypothesis that the events are coming from the beta decay reaction, $\bar{n} \rightarrow \bar{p} \beta^{+} \nu_{e}$, then when the foil is in place, one would have to assume the antineutron must decay near the foil a good fraction of the time. If that is true, then when the foil is removed, there should still be the same event rate of triple coincidences, but there is not. A similar argument can be made about the decay taking place near the vacuum window. Needless to say, this is proof that the incoming particle needs to have a material interaction in order to generate the antihydrogen signature.

Reactions coming from the jet interaction region which could mock the signature for antihydrogen including having the $\bar{p}$ momentum and $e^{+}$pulse height within the correct ranges would also produce just as many events with these variables significantly outside these ranges. It is hard to mock the antihydrogen phase space without filling up a much larger area of phase space with events. No triple coincidences are seen which have $e^{+}$pulse height larger than the expectation from the calibration 
Table 8.1: Number of candidate events summary.

\begin{tabular}{|c||c|c|c|c|}
\hline & $\begin{array}{c}\text { Triple } \\
\text { Coincidences }\end{array}$ & $\begin{array}{c}\text { High Momentum } \\
\text { with Spectators }\end{array}$ & $\begin{array}{c}\text { High Momentum } \\
\mathrm{w} / \mathrm{o}^{+}\end{array}$ & $\begin{array}{c}\int \mathcal{L} d t \\
\left(\mathrm{nb}^{-1}\right)\end{array}$ \\
\hline \hline \multicolumn{5}{|c|}{ Foil Target-In } \\
\hline Above Transition & 72 & 1 & 31 & 77.0 \\
Below Transition & 4 & 1 & 63 & 7.6 \\
\hline \multicolumn{5}{|c|}{ Foil Target-Out } \\
\hline Above Transition & 0 & 20 & 12 & 33.1 \\
Below Transition & 0 & 3 & 62 & 7.0 \\
\hline
\end{tabular}

data. No triple coincidences are seen which have a reconstructed antiproton momentum more than $0.3 \%$ different from the beam momentum whereas $\sim 80 \%$ of the tracks seen at all in the detector are $2-3 \%$ lower than beam momentum.

The conclusion drawn is that antihydrogen has definitely been observed arising from the antiproton-proton interaction studied in this dissertation. Table 8.1 presents the summary number of events under the different experimental conditions.

The candidate events are listed in chronological order in tables 8.1, 8.1, 8.1, 8.1 , and 8.1 along with the integrated luminosity accumulated at the corresponding point. Table 8.1 includes data for 1996. Table 8.1, and 8.1 include data for 1997 up until the one month shutdown when the apertures were expanded, (see section 3.8). Tables 8.1, and 8.1 include the rest of the 1997 data split into two tables for convenience. The foil target column entry in the tables has the meaning, $0 \equiv 1^{\prime \prime}$ empty target (foil-out), $1 \equiv 1 "$ diameter, $430 \mu \mathrm{g} / \mathrm{cm}^{2}$ thick foil, $2 \equiv 1.75$ " diameter, $777 \mu \mathrm{g} / \mathrm{cm}^{2}$ thick foil. The candidates are listed separately as triple coincidences and high momentum track events with spectators. Also listed are the high momentum track events without evidence of a positron. These are not considered as candidates. 
Table 8.2: E862 candidate events and integrated luminosity. A: triple coincidences, $\mathrm{B}$ : high momentum events $\mathrm{w} / \mathrm{o} e^{+}, \mathrm{C}$ : high momentum events with spectator hit.

\begin{tabular}{|c||c|c|c|c|c|c|c|}
\hline Run & $\begin{array}{c}\text { Foil } \\
\text { Target }\end{array}$ & $\begin{array}{c}\text { Momentum } \\
(\mathrm{GeV} / \mathrm{c})\end{array}$ & $\begin{array}{c}\int \mathcal{L} d t \\
\left(\mathrm{nb^{-1 } )}\right.\end{array}$ & Correction & $\mathrm{A}$ & $\mathrm{B}$ & $\mathrm{C}$ \\
\hline 1 & 1 & 5.7261 & 732.36 & 0.317 & 0 & 0 & 0 \\
2 & 1 & 5.7261 & 652.08 & 0 & 0 & 0 & 0 \\
3 & 1 & 5.7261 & 573.46 & 0 & 0 & 0 & 0 \\
4 & 1 & 5.7285 & 713.04 & 0.842 & 0 & 0 & 0 \\
5 & 1 & 5.7285 & 543.57 & 0.807 & 1 & 0 & 0 \\
6 & 1 & 5.7240 & 312.67 & 0.724 & 0 & 2 & 0 \\
7 & 1 & 5.7240 & 674.91 & 0.772 & 2 & 1 & 0 \\
8 & 1 & 5.7209 & 358.70 & 0.833 & 0 & 0 & 0 \\
9 & 1 & 5.6807 & 880.26 & 0.706 & 0 & 0 & 0 \\
10 & 1 & 6.0654 & 1723.31 & 0.85 & 5 & 0 & 0 \\
11 & 1 & 5.9922 & 1637.33 & 0.734 & 1 & 0 & 0 \\
12 & 1 & 6.2335 & 1747.88 & 0.896 & 0 & 0 & 0 \\
13 & 1 & 6.0312 & 1095.89 & 0.78 & 3 & 2 & 0 \\
14 & 1 & 5.9520 & 1538.01 & 0.701 & 3 & 2 & 0 \\
15 & 1 & 5.9100 & 1084.97 & 0.571 & 1 & 0 & 0 \\
16 & 1 & 6.2323 & 571.94 & 0.885 & 0 & 0 & 0 \\
17 & 0 & 6.0093 & 835.32 & 0.951 & 0 & 0 & 0 \\
18 & 1 & 6.0093 & 1104.22 & 0.587 & 0 & 1 & 0 \\
19 & 0 & 5.9747 & 481.25 & 0.729 & 0 & 1 & 1 \\
20 & 1 & 5.9747 & 936.89 & 0.426 & 1 & 0 & 0 \\
\hline
\end{tabular}


Table 8.3: E862 candidate events and integrated luminosity. A: triple coincidences, $\mathrm{B}$ : high momentum events $\mathrm{w} / \mathrm{o} e^{+}, \mathrm{C}$ : high momentum events with spectator hit.

\begin{tabular}{|c||c|c|c|c|c|c|c|}
\hline Run & $\begin{array}{c}\text { Foil } \\
\text { Target }\end{array}$ & $\begin{array}{c}\text { Momentum } \\
(\mathrm{GeV} / \mathrm{c})\end{array}$ & $\begin{array}{c}\int \mathcal{L} d t \\
\left(n b^{-1}\right)\end{array}$ & Correction & $\mathrm{A}$ & $\mathrm{B}$ & $\mathrm{C}$ \\
\hline 21 & 0 & 6.2317 & 603.28 & 0.649 & 1 & 0 & 0 \\
22 & 0 & 5.9366 & 534.98 & 0.78 & 0 & 0 & 0 \\
23 & 1 & 5.9366 & 871.09 & 0.928 & 2 & 2 & 0 \\
24 & 0 & 5.8936 & 766.59 & 0.854 & 0 & 0 & 1 \\
25 & 1 & 5.8936 & 812.49 & 0.952 & 0 & 0 & 0 \\
26 & 0 & 5.7239 & 558.12 & 0.872 & 0 & 1 & 1 \\
27 & 1 & 5.7239 & 768.74 & 0.964 & 0 & 0 & 0 \\
28 & 1 & 6.1090 & 1646.01 & 0.249 & 0 & 0 & 0 \\
28 & 1 & 6.0618 & 1233.31 & 0.136 & 0 & 0 & 0 \\
29 & 1 & 5.8550 & 1474.84 & 0.207 & 0 & 0 & 0 \\
29 & 1 & 5.8201 & 631.53 & 0.096 & 0 & 0 & 0 \\
30 & 1 & 5.8744 & 1433.22 & 0.155 & 0 & 1 & 0 \\
30 & 1 & 5.8163 & 430.55 & 0.833 & 0 & 0 & 0 \\
30 & 1 & 3.5783 & 400.10 & 0.024 & 0 & 3 & 0 \\
31 & 1 & 6.1296 & 1463.78 & 0.859 & 2 & 0 & 0 \\
31 & 1 & 5.8362 & 1505.67 & 0.896 & 2 & 0 & 0 \\
32 & 1 & 6.0951 & 1442.84 & 0.937 & 2 & 1 & 0 \\
33 & 1 & 3.8270 & 489.33 & 0.541 & 0 & 3 & 0 \\
34 & 0 & 6.0498 & 1101.80 & 0.701 & 0 & 0 & 1 \\
35 & 1 & 6.0498 & 363.86 & 0.824 & 0 & 0 & 0 \\
36 & 1 & 5.9235 & 1289.30 & 0.883 & 1 & 1 & 0 \\
37 & 0 & 5.9235 & 518.96 & 0.872 & 0 & 0 & 0 \\
37 & 0 & 5.6472 & 695.51 & 0.81 & 0 & 0 & 0 \\
\hline
\end{tabular}


Table 8.4: E862 candidate events and integrated luminosity. A: triple coincidences, $\mathrm{B}$ : high momentum events $\mathrm{w} / \mathrm{o} e^{+}, \mathrm{C}$ : high momentum events with spectator hit.

\begin{tabular}{|c||c|c|c|c|c|c|c|}
\hline Run & $\begin{array}{c}\text { Foil } \\
\text { Target }\end{array}$ & $\begin{array}{c}\text { Momentum } \\
(\mathrm{GeV} / \mathrm{c})\end{array}$ & $\begin{array}{c}\int \mathcal{L} d t \\
\left(\mathrm{nb^{-1 } )}\right.\end{array}$ & Correction & $\mathrm{A}$ & $\mathrm{B}$ & $\mathrm{C}$ \\
\hline 38 & 1 & 5.6460 & 221.41 & 0.848 & 0 & 1 & 0 \\
38 & 1 & 3.6339 & 285.76 & 0.076 & 1 & 0 & 0 \\
39 & 1 & 3.7006 & 285.76 & 0.872 & 0 & 1 & 0 \\
40 & 1 & 6.2323 & 957.91 & 0.87 & 1 & 0 & 0 \\
40 & 1 & 5.8019 & 1605.78 & 0.815 & 1 & 0 & 0 \\
41 & 1 & 3.6770 & 249.94 & 0.463 & 0 & 0 & 0 \\
42 & 1 & 3.6762 & 399.10 & 0.37 & 1 & 2 & 0 \\
43 & 0 & 5.5765 & 1006.14 & 0.489 & 0 & 0 & 1 \\
44 & 1 & 5.5765 & 970.23 & 0.907 & 2 & 0 & 0 \\
45 & 0 & 5.5168 & 1125.03 & 0.922 & 0 & 0 & 1 \\
46 & 1 & 4.0662 & 491.92 & 0.764 & 0 & 0 & 0 \\
47 & 1 & 5.7315 & 1024.91 & 0.911 & 0 & 0 & 0 \\
48 & 1 & 5.7315 & 900.76 & 0.923 & 0 & 0 & 0 \\
48 & 1 & 5.6098 & 1676.50 & 0.762 & 3 & 1 & 0 \\
48 & 1 & 5.5530 & 453.10 & 0.739 & 0 & 0 & 0 \\
49 & 1 & 5.6104 & 3149.58 & 0.759 & 2 & 2 & 0 \\
50 & 1 & 5.6100 & 578.00 & 0.905 & 1 & 1 & 0 \\
50 & 1 & 5.5551 & 319.68 & 0.869 & 0 & 0 & 0 \\
50 & 1 & 5.5513 & 314.50 & 0.889 & 0 & 0 & 0 \\
50 & 1 & 5.5460 & 303.97 & 0.87 & 0 & 0 & 0 \\
\hline
\end{tabular}


Table 8.5: E862 candidate events and integrated luminosity. A: triple coincidences, $\mathrm{B}$ : high momentum events $\mathrm{w} / \mathrm{o} e^{+}, \mathrm{C}$ : high momentum events with spectator hit.

\begin{tabular}{|c||c|c|c|c|c|c|c|}
\hline Run & $\begin{array}{c}\text { Foil } \\
\text { Target }\end{array}$ & $\begin{array}{c}\text { Momentum } \\
(\mathrm{GeV} / \mathrm{c})\end{array}$ & $\begin{array}{c}\int \mathcal{L} d t \\
\left(n b^{-1}\right)\end{array}$ & $\begin{array}{c}\text { Correction } \\
\mathrm{A}\end{array}$ & $\mathrm{B}$ & $\mathrm{C}$ \\
\hline 51 & 2 & 6.2300 & 112.02 & 0.754 & 0 & 0 & 0 \\
52 & 2 & 6.2300 & 3.41 & 0.735 & 0 & 0 & 0 \\
53 & 2 & 3.5790 & 672.60 & 0.898 & 0 & 0 & 0 \\
54 & 2 & 3.5790 & 586.49 & 0.895 & 0 & 6 & 0 \\
55 & 2 & 6.2300 & 1155.45 & 0.882 & 6 & 1 & 0 \\
56 & 2 & 6.2300 & 303.38 & 0.864 & 2 & 0 & 0 \\
57 & 2 & 3.6320 & 302.58 & 0.902 & 0 & 3 & 0 \\
58 & 2 & 3.6320 & 27.47 & 0.901 & 0 & 0 & 0 \\
59 & 2 & 3.6320 & 31.16 & 0.915 & 0 & 0 & 0 \\
60 & 0 & 3.6526 & 0.00 & 0 & 0 & 6 & 0 \\
61 & 0 & 3.6526 & 788.67 & 0.974 & 0 & 11 & 1 \\
62 & 0 & 4.0650 & 328.29 & 0.926 & 0 & 9 & 0 \\
63 & 0 & 3.4554 & 190.21 & 0.885 & 0 & 4 & 0 \\
64 & 0 & 3.7562 & 1111.93 & 0.924 & 0 & 10 & 1 \\
65 & 0 & 3.5987 & 379.37 & 0.87 & 0 & 6 & 0 \\
66 & 0 & 3.5987 & 600.10 & 0.879 & 0 & 10 & 0 \\
67 & 0 & 3.5987 & 374.17 & 0.918 & 0 & 4 & 0 \\
68 & 2 & 3.4530 & 514.68 & 0.923 & 0 & 9 & 0 \\
69 & 2 & 3.5130 & 855.31 & 0.955 & 1 & 9 & 0 \\
70 & 2 & 3.6926 & 1389.60 & 0.983 & 0 & 18 & 1 \\
71 & 2 & 3.6855 & 639.00 & 0.936 & 1 & 9 & 0 \\
72 & 2 & 5.7240 & 789.79 & 0.986 & 0 & 0 & 1 \\
73 & 2 & 5.7240 & 1132.23 & 0.954 & 1 & 1 & 0 \\
74 & 2 & 5.6200 & 1388.78 & 0.935 & 1 & 1 & 0 \\
75 & 0 & 5.6100 & 968.74 & 0.777 & 0 & 1 & 0 \\
76 & 0 & 5.6100 & 0.00 & 0 & 0 & 0 & 0 \\
77 & 0 & 5.6100 & 0.00 & 0 & 0 & 0 & 0 \\
78 & 2 & 5.6100 & 3094.13 & 0.951 & 2 & 0 & 0 \\
79 & 0 & 5.6130 & 1396.10 & 0.64 & 0 & 0 & 2 \\
80 & 2 & 5.6110 & 649.03 & 0.982 & 1 & 0 & 0 \\
\hline
\end{tabular}


Table 8.6: E862 candidate events and integrated luminosity. A: triple coincidences, $\mathrm{B}$ : high momentum events $\mathrm{w} / \mathrm{o} e^{+}, \mathrm{C}$ : high momentum events with spectator hit.

\begin{tabular}{|c||c|c|c|c|c|c|c|}
\hline Run & $\begin{array}{c}\text { Foil } \\
\text { Target }\end{array}$ & $\begin{array}{c}\text { Momentum } \\
(\mathrm{GeV} / \mathrm{c})\end{array}$ & $\begin{array}{c}\text { J Ldt } \\
\left(\mathrm{nb} \mathrm{b}^{-1}\right)\end{array}$ & $\begin{array}{c}\text { Correction } \\
\mathrm{A}\end{array}$ & $\mathrm{B}$ & $\mathrm{C}$ \\
\hline 81 & 2 & 5.6050 & 3255.56 & 0.959 & 1 & 3 & 0 \\
82 & 2 & 5.6080 & 2291.40 & 0.937 & 0 & 0 & 0 \\
83 & 2 & 5.6083 & 3308.60 & 0.95 & 4 & 0 & 0 \\
84 & 2 & 5.6042 & 2902.98 & 0.961 & 5 & 1 & 0 \\
85 & 2 & 5.6072 & 2356.17 & 0.95 & 1 & 0 & 0 \\
86 & 2 & 5.6072 & 70.63 & 0.982 & 1 & 0 & 0 \\
87 & 2 & 5.6072 & 1590.01 & 0.981 & 0 & 1 & 0 \\
88 & 2 & 5.2035 & 112.21 & 0.84 & 0 & 0 & 0 \\
89 & 2 & 5.6019 & 3651.98 & 0.997 & 6 & 3 & 0 \\
90 & 2 & 5.6053 & 1076.58 & 0.966 & 1 & 0 & 0 \\
91 & 2 & 5.6053 & 241.74 & 0.965 & 0 & 0 & 0 \\
92 & 0 & 5.6053 & 1802.45 & 0.393 & 0 & 2 & 0 \\
93 & 0 & 5.6068 & 3917.12 & 0.387 & 0 & 1 & 0 \\
94 & 0 & 5.6095 & 3229.01 & 0.333 & 0 & 2 & 2 \\
95 & 0 & 5.5552 & 2239.63 & 0.362 & 0 & 1 & 0 \\
96 & 0 & 4.4500 & 0.00 & 0 & 0 & 0 & 0 \\
97 & 0 & 6.2300 & 1825.21 & 0.311 & 0 & 0 & 1 \\
98 & 0 & 6.3914 & 979.68 & 0.342 & 0 & 0 & 0 \\
98 & 0 & 5.2035 & 692.03 & 0.359 & 0 & 0 & 0 \\
99 & 0 & 6.0245 & 2572.89 & 0.285 & 0 & 0 & 2 \\
100 & 0 & 5.1933 & 221.40 & 0.25 & 0 & 0 & 0 \\
101 & 0 & 5.1933 & 584.95 & 0.242 & 0 & 0 & 0 \\
102 & 0 & 5.1625 & 71.08 & 0.234 & 0 & 0 & 0 \\
103 & 0 & 5.1650 & 884.08 & 0.243 & 0 & 0 & 0 \\
104 & 0 & 4.0795 & 137.86 & 0.246 & 0 & 0 & 0 \\
105 & 0 & 5.5516 & 772.33 & 0.267 & 0 & 0 & 0 \\
106 & 0 & 5.5516 & 1080.81 & 0.275 & 0 & 0 & 0 \\
107 & 0 & 4.8455 & 931.45 & 0.268 & 0 & 1 & 0 \\
108 & 2 & 6.0226 & 1863.90 & 0.947 & 2 & 2 & 0 \\
109 & 2 & 5.2628 & 349.12 & 0.805 & 1 & 0 & 0 \\
110 & 0 & 5.2480 & 387.12 & 0.867 & 0 & 1 & 1 \\
111 & 0 & 8.8150 & 754.54 & 0.894 & 0 & 0 & 3 \\
112 & 0 & 6.9343 & 870.29 & 0.876 & 0 & 0 & 1 \\
113 & 0 & 5.7231 & 1475.59 & 0.977 & 0 & 1 & 2 \\
114 & 0 & 5.4349 & 500.45 & 0.958 & 0 & 1 & 0 \\
115 & 0 & 4.6686 & 409.00 & 0.912 & 0 & 1 & 1 \\
\hline
\end{tabular}




\subsection{Cross Section for $6 \mathrm{GeV} / \mathrm{c}$}

The different theoretical estimates of the cross section outlined in section 1.3, (shown in figures 1.5, and 1.6) differ in the Accumulator energy region by up to $30 \%$ even though the errors on the individual calculations are small. The wavefunctions used to calculate the cross section are known hydrogen wavefunctions. Some error may arise because the equivalent photon method is designed to be used at somewhat higher energies than these so the electric field of the nucleus is not quite transverse to the antiproton trajectory.

From now on in the cross section discussion, it is assumed that runs $1-13,51$, 68 , and 75-79 are not considered in the calculated and fitted numbers. The reasons are outlined in section 6.6 .

An attempt was made to see whether the acceptance and efficiency corrections were reasonable. If all the proper corrections have been applied, the candidate distribution, when grouped into bins of equal sensitivity should reproduce a Poisson distribution since only the counting statistics are involved. Sensitivity is defined as the integrated luminosity multiplied by all the corrections for acceptance and effciency. The peak of the fitted Poisson distribution will not neccessarily correspond to the average cross section since the number of events is small. Figures 8.1 , and 8.2 show the candidates grouped into bins of equal sensitivity. Only triple coincidence events above transition were considered. The second of the two plots considers a subset of these which have good tracks. The first set has 57 events. The second set includes 52 events. Table 8.7 compares the numbers for cross section near $6 \mathrm{GeV} / \mathrm{c}$ found from the peak of the Poisson distribution and from simply averaging the events. 


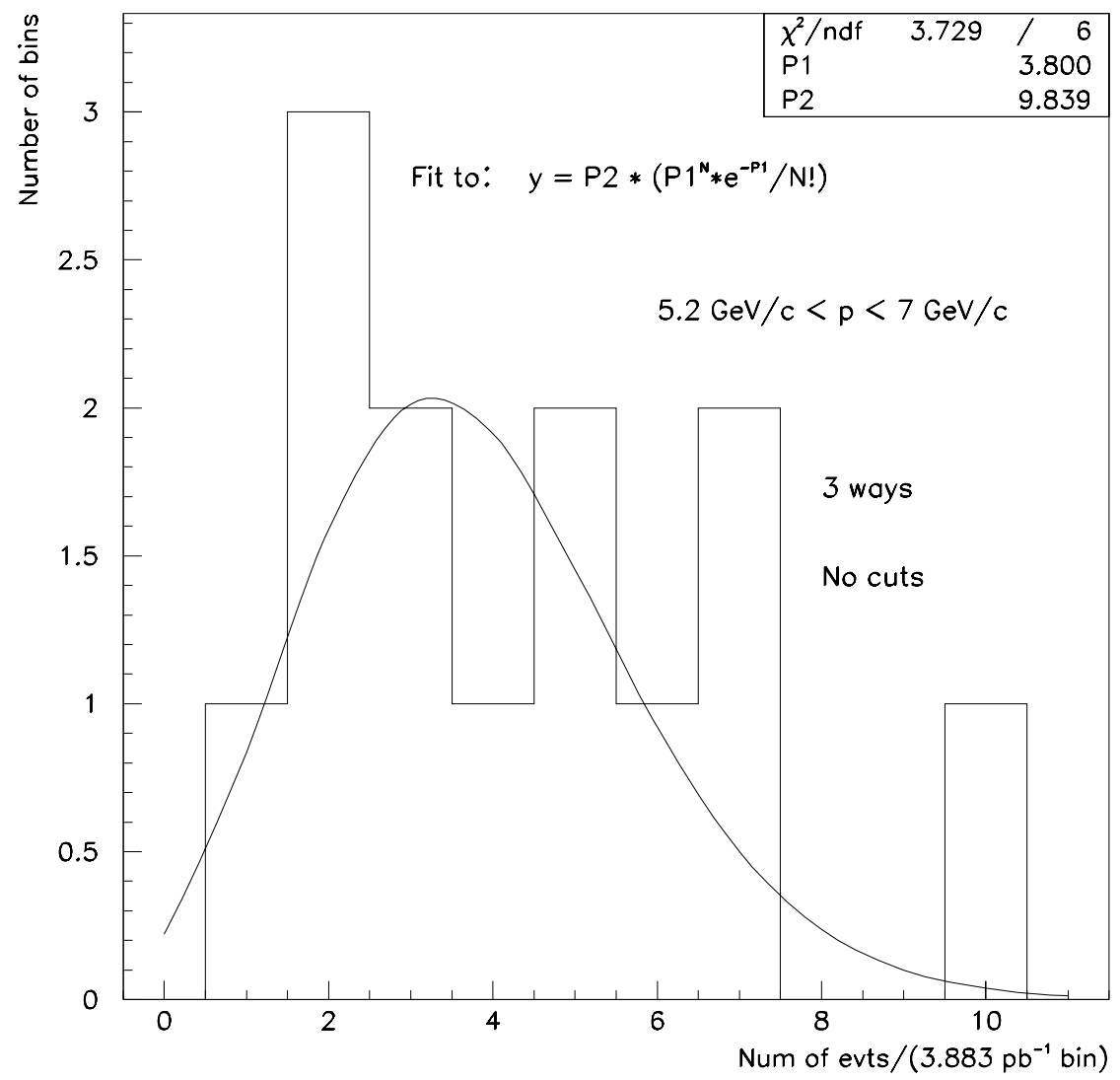

Figure 8.1: Number of events in equal sensitivity bins. All triple coincidences are considered. The curve shown is a Poisson distribution fitted to the data.

Table 8.7: Comparison of cross section $\left(p b^{-1}\right)$ estimates.

\begin{tabular}{|c||c|c|}
\hline & No cuts & Good Track only \\
\hline Binned & 0.979 & 1.09 \\
Average & 1.129 & 1.03 \\
\hline
\end{tabular}




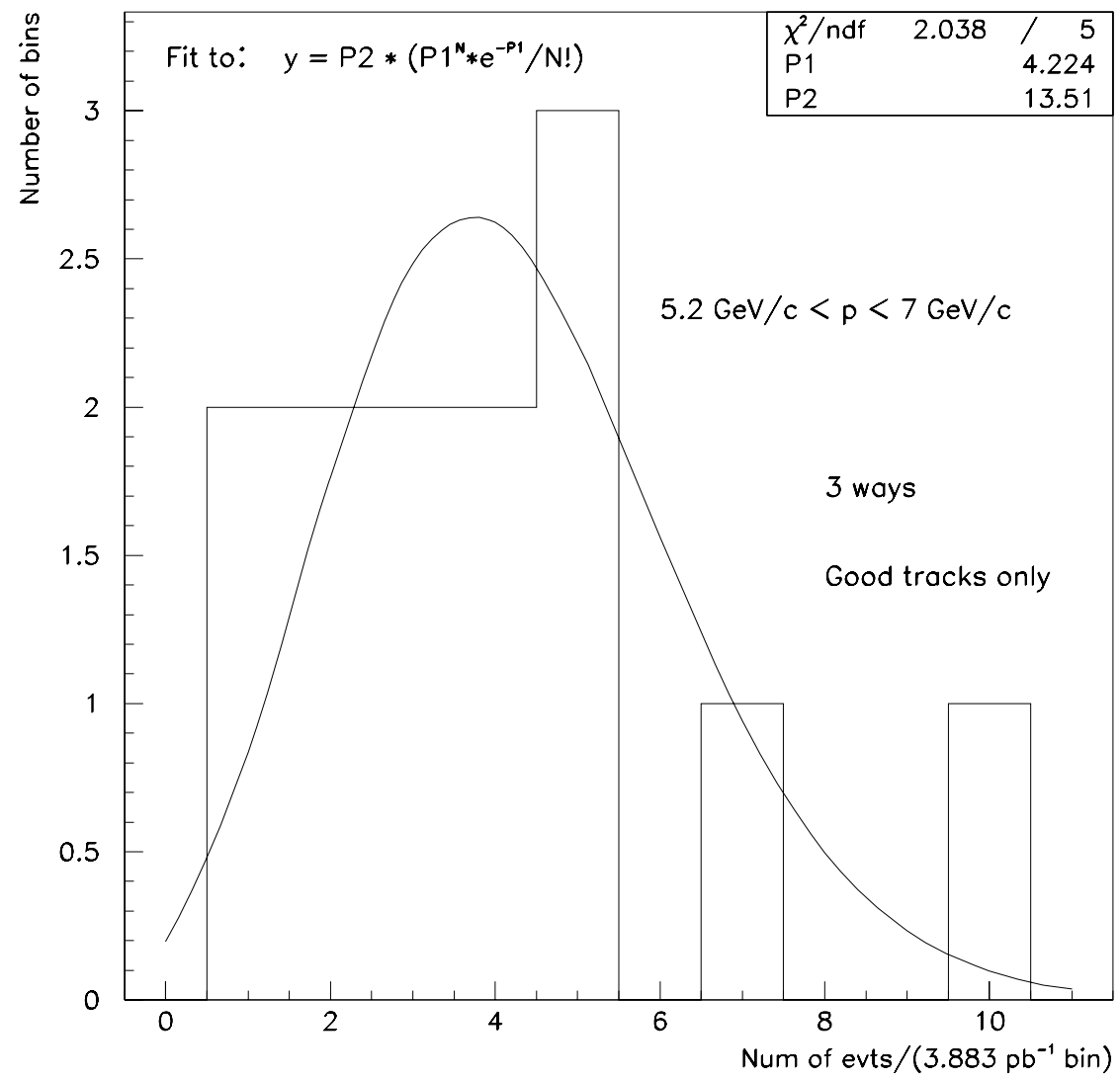

Figure 8.2: Number of events in equal sensitivity bins. Only triple coincidences with a good track are considered. The curve shown is a Poisson disttribution fitted to the data. 
A maximum likelihood fit was made to the data by assuming the number of candidate events followed a Poisson distribution. The expected mean of the Poisson distribution was set equal to the product of the integrated luminosity $\times$ corrections $\times$ expected cross section:

$$
\mu_{i}=\left(\int \mathcal{L} d t\right)_{i} \times \epsilon_{i} \times \sigma_{e x p}\left(P_{\bar{p}, i}\right)
$$

Each run-momentum combination was used separately as a datum point. Triple coincidence data and foil-out data were fitted separately. For each, fits were made with both above and below transition data, and with above transition data only.

For the expected cross section as a function of momentum, the form derived by Munger, Brodsky, and Schmidt[47] was used. The constant multiplier which they calculated to be $f=1.42$ was allowed to be a freely adjustable parameter in the fit. The function of momenta comes purely from the equivalent photon method.

$$
\sigma_{e x p}\left(P_{\bar{p}}, i\right)=f \times\left(\ln \left(\gamma^{2}+1\right)-\frac{\gamma^{2}}{\left(\gamma^{2}+1\right)}\right), \gamma=\sqrt{P_{\bar{p}, i}^{2}+m_{\bar{p}}^{2}}
$$

The maximum likelihood function is a product of $\mathrm{N}$ Poisson functions,

$$
\Lambda=\prod_{i=1}^{N} \frac{\mu_{i}^{n_{i}} e^{-\mu_{i}}}{n_{i} !}
$$

where $n_{i}$ is the number of events observed for the $i^{\text {th }}$ datum point. The log-likelihood function, $-\ln (\Lambda)$, is maximized resulting in fitted values for the factor, $f$. Table 8.8 shows the resulting values for $f$ and the corresponding cross section at $6 \mathrm{GeV} / \mathrm{c}$. 
Table 8.8: Results of maximum likelihood fits. Cross section is in pb. $\delta$ Mean $=$ $\sigma_{8-1} / \sqrt{8}$

\begin{tabular}{|l||c|c|}
\hline & $f$ & $\sigma(P=6 \mathrm{GeV} / \mathrm{c})$ \\
\hline Foil-in & $0.4215_{-0.0712}^{+0.0803}$ & $1.1727_{-0.198}^{+0.223}$ \\
Foil-in, good track only & $0.4080_{-0.0700}^{+0.0790}$ & $1.1351_{-0.195}^{+0.220}$ \\
Foil-in, above transition & $0.4241_{-0.0739}^{+0.0838}$ & $1.1798_{-0.206}^{+0.233}$ \\
Foil-in, above transition, & $0.4169_{-0.0733}^{+0.0831}$ & $1.1597_{-0.204}^{+0.231}$ \\
$\quad$ good track only & & \\
Foil-in, above transition, & $0.3852_{-0.0734}^{+0.0841}$ & $1.0717_{-0.204}^{+0.234}$ \\
$\quad$ runs 52-74 removed & & \\
Foil-in, above transition, & $0.3773_{-0.0726}^{+0.0832}$ & $1.0496_{-0.202}^{+0.231}$ \\
$\quad$ runs 52-74 removed & & \\
$\quad$ good track only & & $1.1374_{-0.303}^{+0.369}$ \\
Foil-out & $0.4089_{-0.1090}^{+0.1327}$ & $1.1840_{-0.336}^{+0.415}$ \\
\hline Foil-out, above transition & $0.4256_{-0.1207}^{+0.1492}$ & $1.1363 \pm 0.018$ \\
\hline Mean $\pm \delta$ Mean & & \\
\hline
\end{tabular}




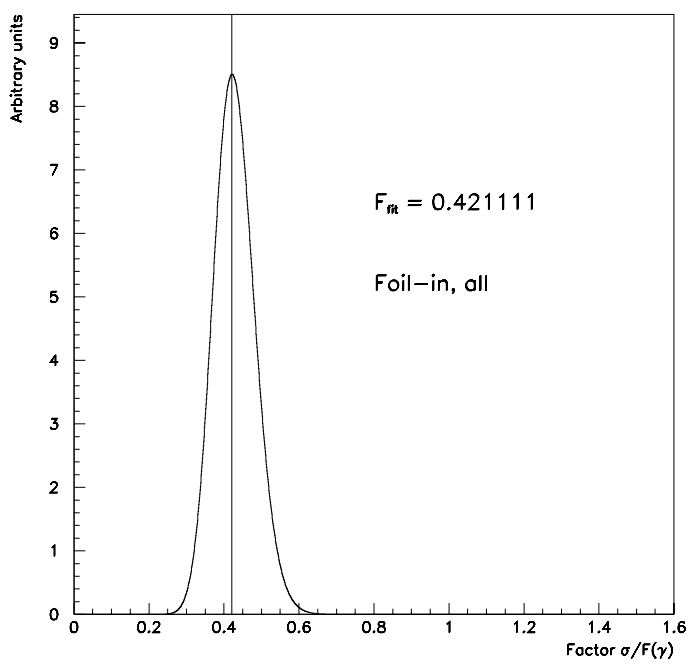

Figure 8.3: Maximum likelihood function (unnormalized), $-\ln (\Lambda)$, as a function of the unknown factor, $f$. Events are foil-out only.

Figures $8.3,8.4,8.5,8.6$, and 8.7 show the maximum likelihood function resulting. The values listed are the location, $f$, of the peak.

\subsection{Energy Dependence of the Cross Section}

Figure 8.8 shows the events per luminosity displayed as a function of beam momentum. No corrections have been made to the data. Figures 8.9 , and 8.10 show the cross section which is the events per luminosity with corrections taken into account.

Figure 8.11 shows the expected functions for the theoretically predicted antihydrogen production cross section from theory and measured data binned where measurements have been binned together in bins of momentum to increase statistics of the bins. The cross section measured by PS210 at $1.94 \mathrm{GeV} / \mathrm{c}$ is calculated from 


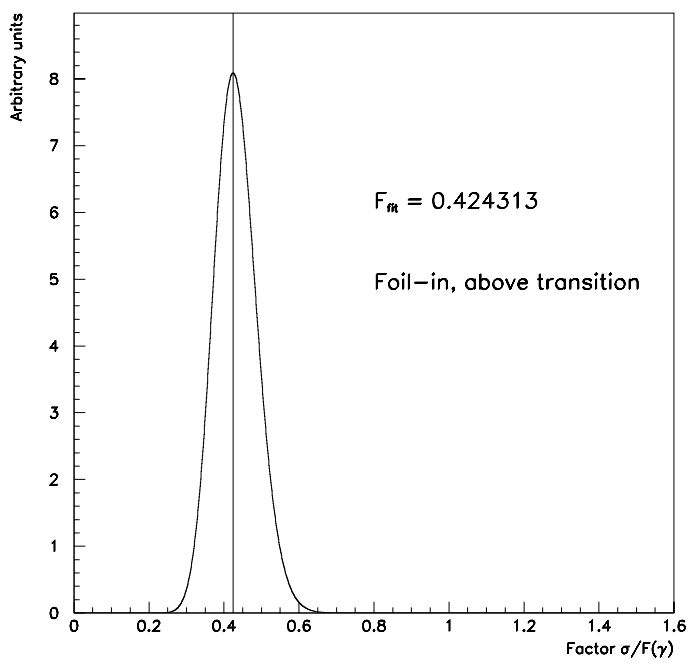

Figure 8.4: Maximum likelihood function (unnormalized), $-\ln (\Lambda)$, as a function of the unknown factor, $f$. Events are foil-in. above transition only.

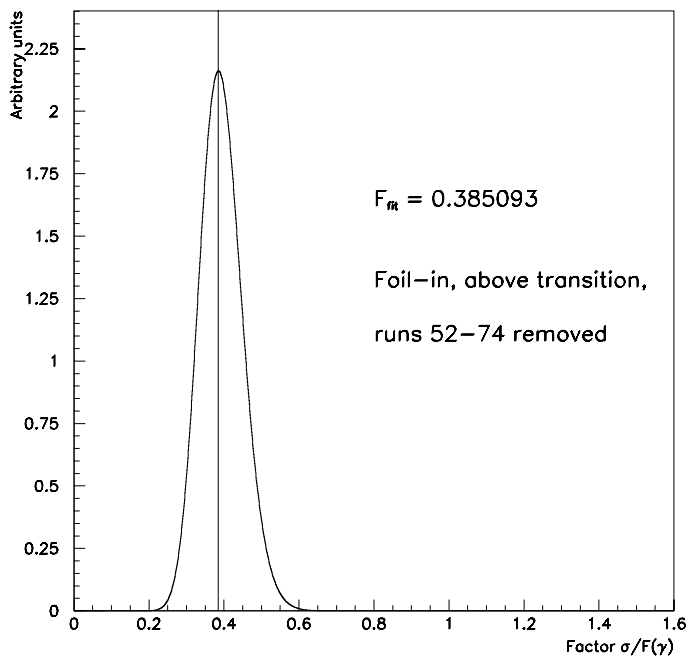

Figure 8.5: Maximum likelihood function (unnormalized), $-\ln (\Lambda)$, as a function of the unknown factor, $f$. Events are foil-in, above transition only, with certain runs not considered. 


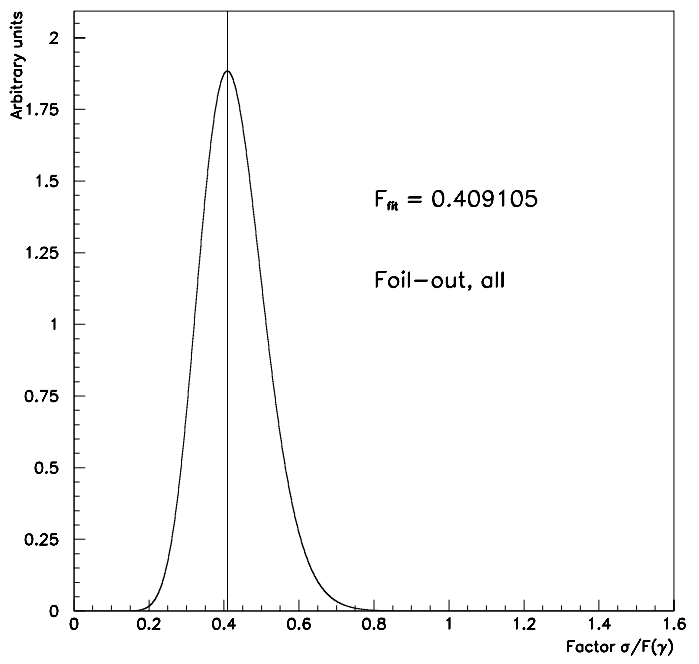

Figure 8.6: Maximum likelihood function (unnormalized), $-\ln (\Lambda)$, as a function of the unknown factor, $f$. Events are foil-out only.

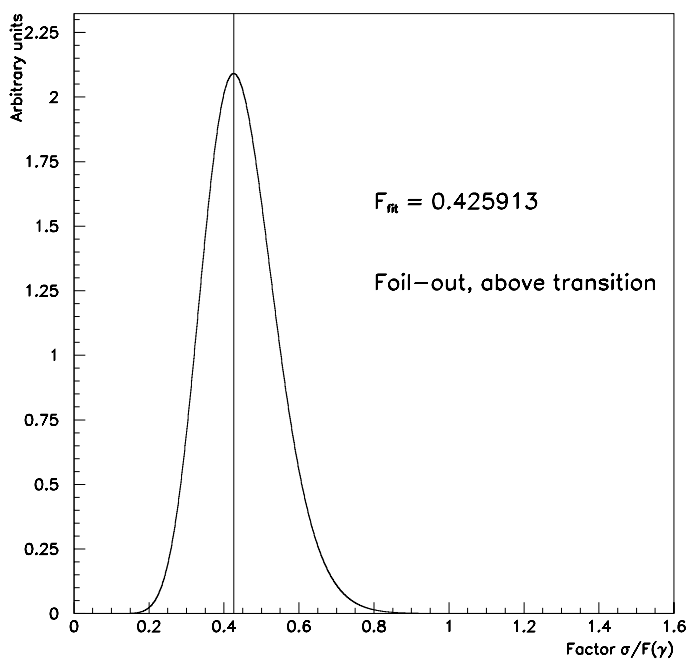

Figure 8.7: Maximum likelihood function (unnormalized), $-\ln (\Lambda)$, as a function of the unknown factor, $f$. Events are foil-out, above transition only. 


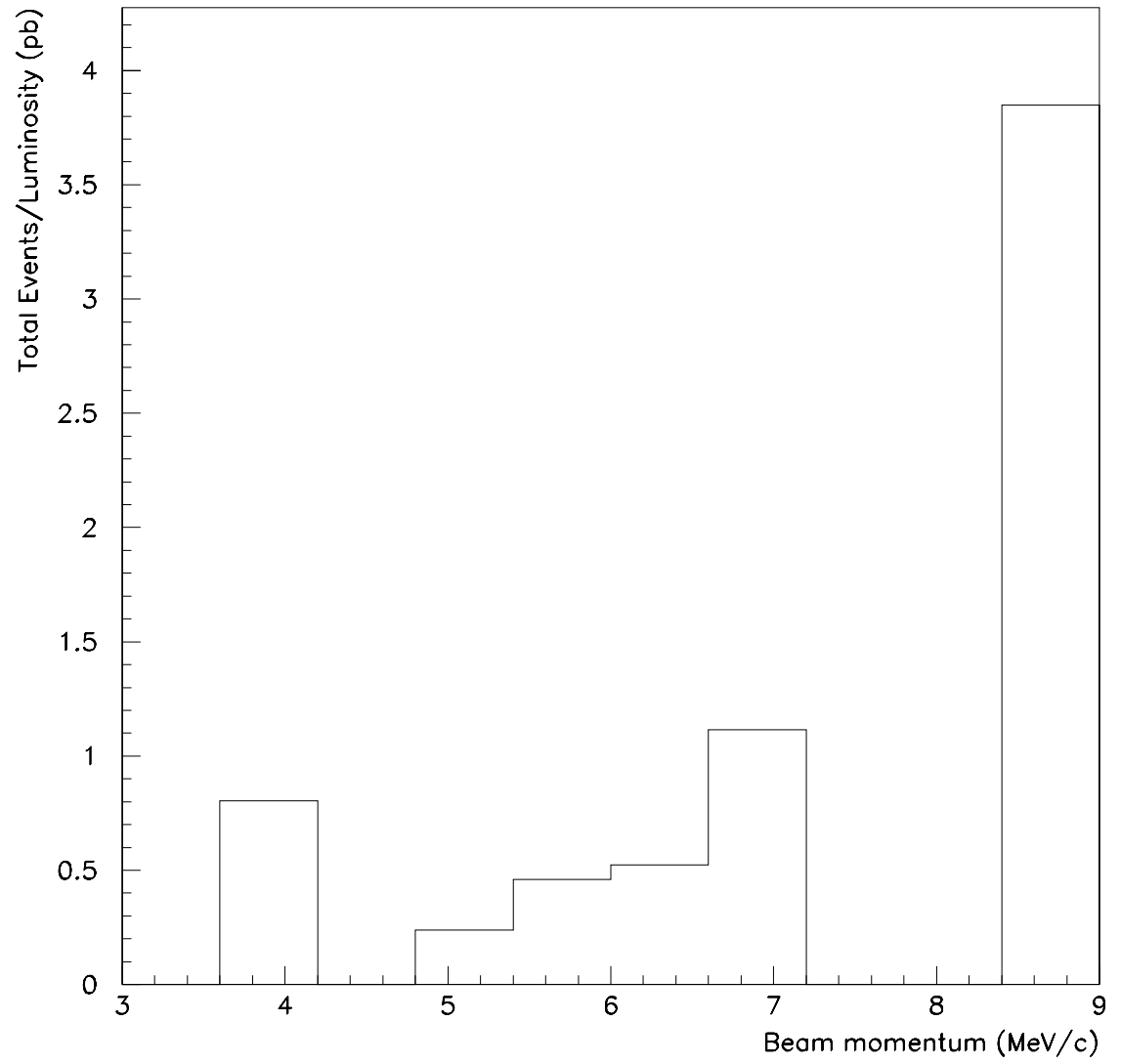

Figure 8.8: Events per luminosity using 10 bins in momentum. 


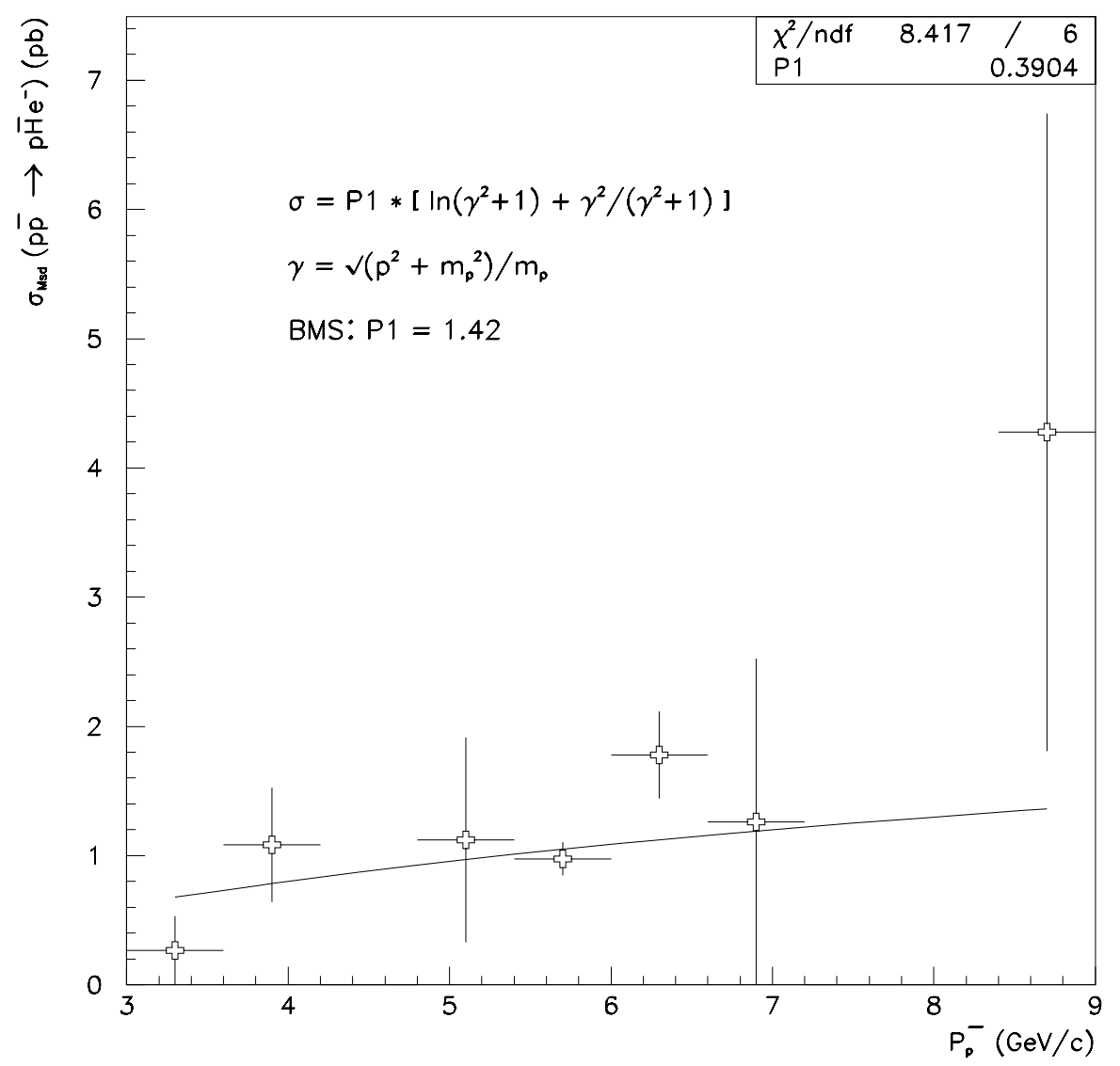

Figure 8.9: Cross section using 10 bins in momentum. The line represents a $\chi^{2}$ fit to $\mathrm{F}(\gamma)$ from $[47]$. 


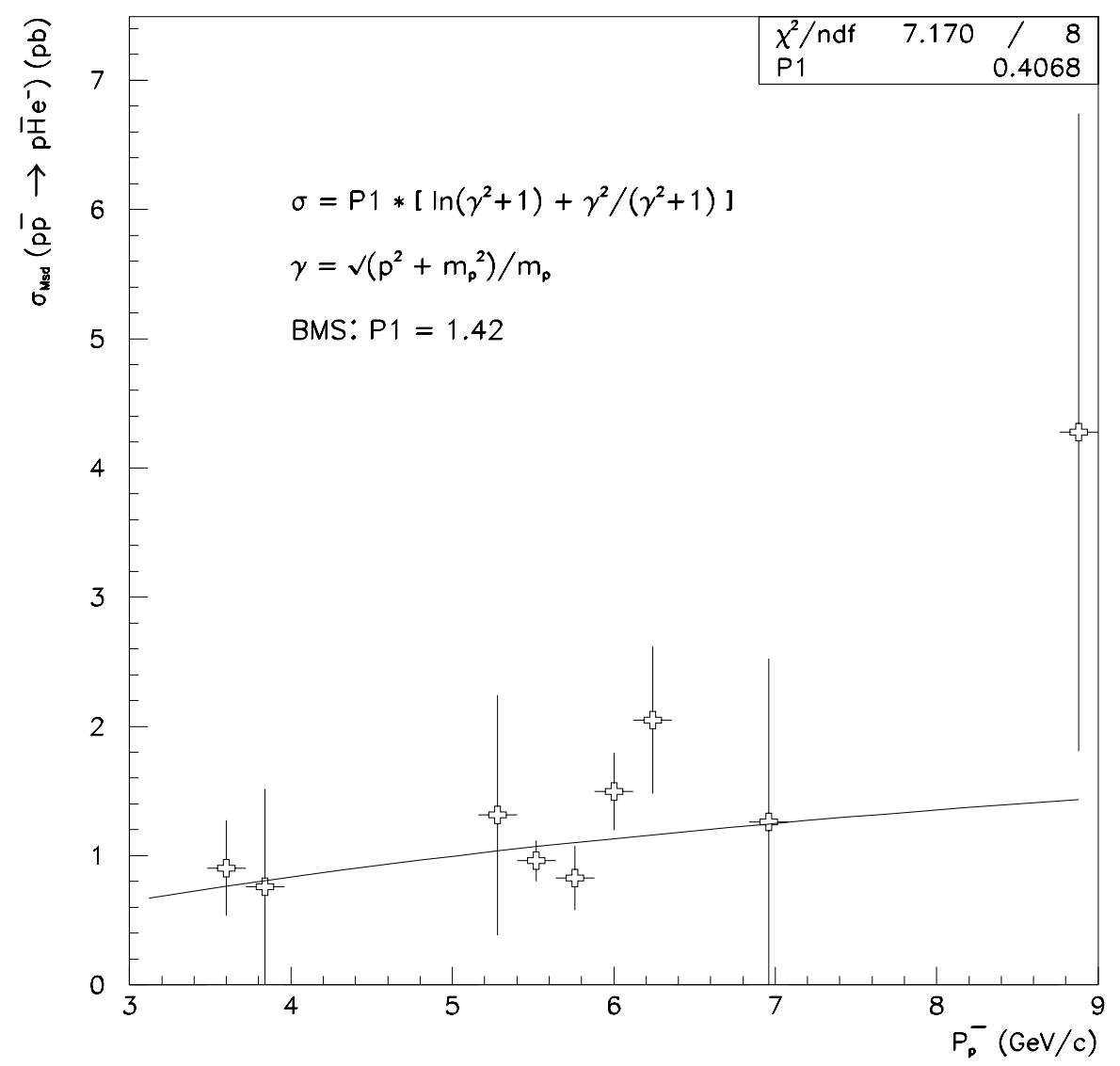

Figure 8.10: Cross section using 25 bins in momentum. The line represents a $\chi^{2}$ fit to $\mathrm{F}(\gamma)$ from [47]. 
the values given in [5]. Namely, 11 candidate events were observed. Two were determined to be background. The rest were assumed to be antihydrogens. With the statistical error and error on integrated luminosity, their cross section measurement normalized from xenon $(\mathrm{Z}=54)$ to hydrogen is:

$$
\sigma\left(P S 210, P_{\bar{p}}=1.94 G e V / c\right)=2.058 \pm 1.029 \pm 0.686 p b
$$

The PS210 result agrees with the theory quite well considering the large error bars. There is still a question to be answered as to why E862's measured cross section is consistently lower than expectations. The luminosity integrated by E862 is known very well so either the antihydrogen cross section has a different dependence than predicted or some unknown acceptance problem was encountered. 


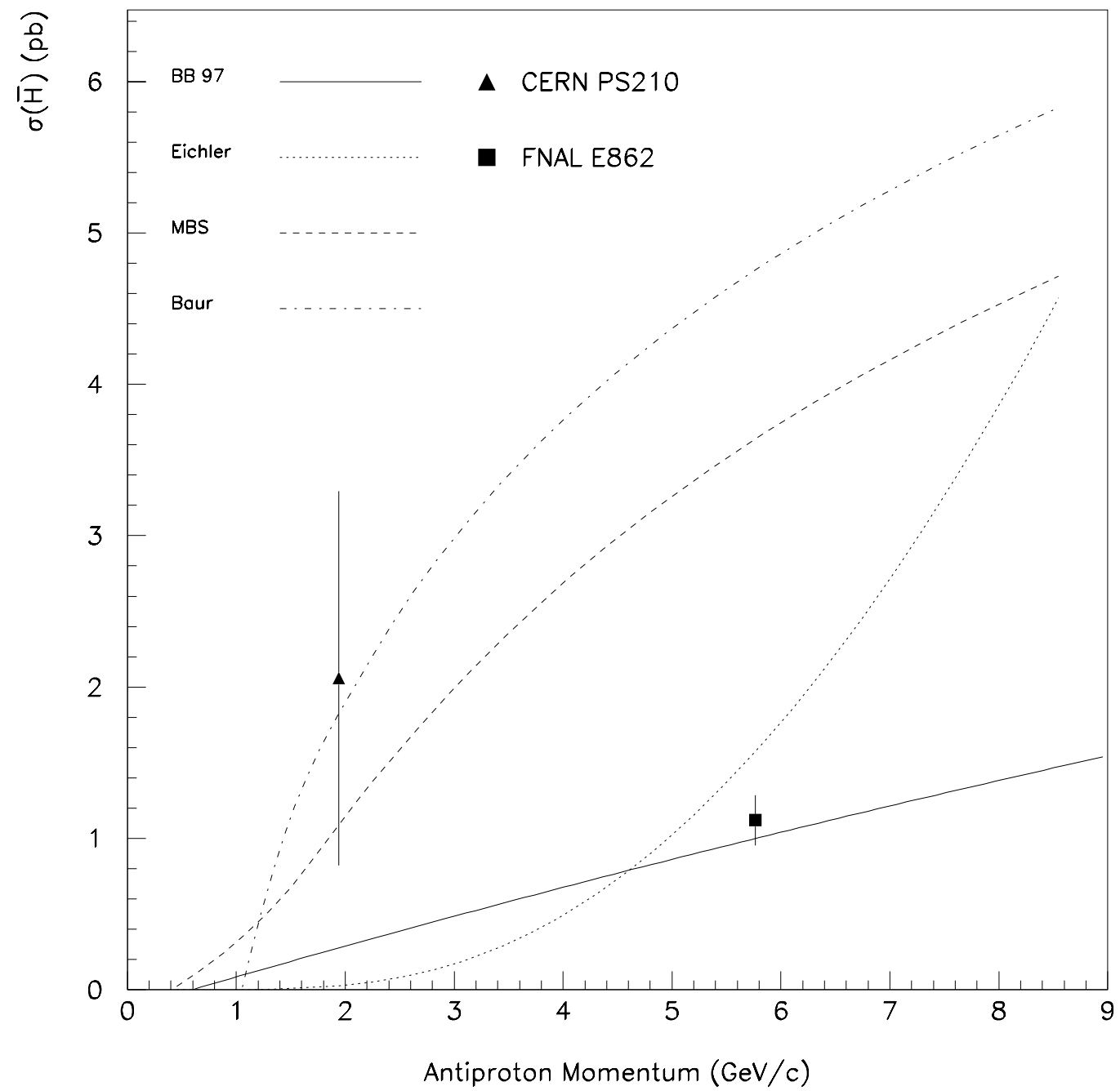

Figure 8.11: Antihydrogen production cross section. Curves are theoretical functions from (MBS)[47], (Baur)[48], (BB 97)[57], and (Eichler)[63]. Data points are from [5] and the present results $[93]$. 


\subsection{Systematic Errors}

The systematic errors in the cross section measurement arise because of imprecise knowledge of the integrated luminosity ( $4 \%$ mostly from the error on the active area of the detector) and and from assumptions on the acceptance calculation. The systematic error in the acceptance estimate is dominated by how well the emittance, and hence the beam size, is known. Another smaller effect is the position of the target wheel. We allowed a possible $2^{\circ}$ rotation since the video camera, which monitored the optical readout of the wheel's angle setting, provided a resolution no better than $2^{\circ}$. Such effects as a possible, small momentum scale error or error in the track's reconstructed position at the jet, etc. had no effect on the cross section since no candidates as chosen by the fundamental signatures, (triple coincidence or track with spectator hit in PWC \#1), had a momentum or position at the jet far significantly outside the acceptable range. The error arising from Monte Carlo statistics was small $(<2 \%)$. The systematic change to the acceptance $\times$ efficiency was calculated by running the simulations described in chapter 7 with the assumed offsets. The assumed offsets were a $2^{\circ}$ change in target wheel angle and a $50 \%$ increase in emittance envelopes in both $\mathrm{x}$ and $\mathrm{y}$ dimensions. Possible beam position measurement error is comparably small. This amounted to a roughly $10 \%$ change in the cross section. One effect which was difficult to model was the correct positions of the $e^{+}$spectrometer magnets. The magnets had been moved significantly from their original, ideal positions. On the other hand, they were moved to correct for imperfections in the construction of the magnets themselves. Thus the empirical success in pointing the test beam of $e^{-}$ on the scintillator, as described in chapter 3, shows that the acceptance of the $e^{+}$ spectrometer cannot be much different than the ideal scenario. 
Table 8.9: Cross section results for different foil thicknesses. The data sets chosen were slightly different than that used in the previous analysis.

\begin{tabular}{|c||c|c|c|c|c|}
\hline $\begin{array}{c}\text { Data } \\
\text { Set }\end{array}$ & $\begin{array}{c}\text { Number } \\
\text { of } \\
\text { Events }\end{array}$ & $\begin{array}{c}\text { Integ. } \\
\text { Lumin. } \\
\left(\mathrm{pb}^{-1}\right)\end{array}$ & $\begin{array}{c}\text { Cross } \\
\text { Section } \\
(\mathrm{pb})\end{array}$ & $\begin{array}{c}\text { Stat. } \\
\text { Error } \\
(\mathrm{pb})\end{array}$ & $\begin{array}{c}\text { Syst. } \\
\text { Error } \\
(\mathrm{pb})\end{array}$ \\
\hline $437 \mu \mathrm{g} / \mathrm{cm}^{2}$ foil & 24 & 34.8 & 1.03 & 0.21 & 0.08 \\
$777 \mu \mathrm{g} / \mathrm{cm}^{2}$ foil & 33 & 29.6 & 1.22 & 0.21 & 0.09 \\
No foil & 10 & 11.3 & 1.08 & 0.35 & 0.12 \\
All data & 67 & 75.7 & 1.12 & 0.14 & 0.09 \\
\hline
\end{tabular}

The effect of the combined error from the integrated luminosity and acceptance are included in table where the cross section is calculated for a somewhat different breakup of the data as outlined in [93]. 


\subsection{Conclusions}

In conclusion, antihydrogen atoms have unquestionably been observed. Two different signatures have signalled the antihydrogen, one with the foil target in place, (triple coincidence events), and the other with the foil out of the way (high momentum with spectator hit events). Both types of events prove completely consistent with the antihydrogen hypothesis when each of the kinematic distributions are viewed. A cross section measurement has been made for those events around $6 \mathrm{GeV} / \mathrm{c}$ where there is a large number of events. The cross section is for antihydrogen production at $P_{\bar{p}}=5.767 \mathrm{GeV} / \mathrm{c}, \sigma=1.14 \mathrm{pb}$. The cross section does not agree with the theoretical cross section for this momentum $(\sigma=\sim 3.8 \mathrm{pb})$ [47]. A measurement of the energy dependence of the cross section has been made although, because of much lower statistics at the extreme beam momenta of the range available (3 - 9 $\mathrm{GeV} / \mathrm{c}$ ), agreement with the form of the theoretical cross section is consistent but not definitive. A monotonic function increasing with momentum is clear though.

The results presented here are in partial agreement with the results of CERN PS210[5], (see section 1.4). The observation of antihydrogen has been made at similar, although larger, momenta. The signature of the events is similar. Certain advantages of E862 are the better antiproton momentum resolution, further separation of the $e^{+}$ spectrometer from the antiproton beamline, much further removal of the apparatus from backgrounds, and measurement ability over a range of beam momenta.

These achievements would not have been possible without recognizing the continually improving ability to collect and cool antiprotons over the past 20 years. The progress of the antiproton storage rings at Fermilab and CERN has allowed stacks of greater than $10^{11} \bar{p}$ to be collected for experimental study. Even so, a rate of only one or two antihydrogens per day could be measured. A higher production rate will 
be required to study the system with any precision. The technology of thin gas jets is also needed in order to insure the beam can recirculate but still produce enough antihydrogen to be measured.

It is true that other production approaches should come to fruition soon, their advantages being larger formation rates and ability to perform much more precise spectroscopy on the atomic system. On the other hand, as outlined in Appendix 2, we can perform atomic spectroscopy with higher energy antihydrogen as well. The Lamb shift splitting can be directly measured as a way to probe for CPT-violating interactions. If they exist, they could ultimately alter our understanding of the Standard Model of particle physics. 


\section{Bibliography}

[1] Dirac, P.A.M., 1928, Proc. Roy. Soc., A117, 610.

[2] Dirac, P.A.M., 1931, Proc. Roy. Soc., A133, 60.

[3] Anderson, C.D., Phys. Rev., 43, 491, 1932; Science, 76, 238, 1932.

[4] Chamberlain, O., Segre, E., Wiegand, C., Ysilantis, T., Phys. Rev., 100, 947, 1955.

[5] G. Baur et al., Production of Anti-Hydrogen, Phys. Lett., B 368, 251, 1996.

[6] G. Luders, Det. Kon. Dan. Vid. Sel. Mat.fys Medd., 28, 5, 1954.; G. Luders, Ann. Phys., 2, 1957.

[7] W. Pauli, in: Niels Bohr and the development of physics, ed. W. Pauli (Pergamon, New York), 30, 1955.

[8] J.S. Bell, Proc. Roy. Soc., A 231, 479, 1955.

[9] R. Jost, The general theory of quantized fields, (American Mathematical Society, Providence, Rhode Island), 1965.

[10] D. Colladay and V.A. Kostelecky, CPT violation and the standard model, Phys. Rev., D 55, 11, 1997.

[11] J. Ellis, N.E. Mavromatos, and D.V. Nanopoulos, CPT violation in stringmodified quantum mechanics and the neutral kaon system, Int. J. Mod. Phys., A 11, 1489, 1996. 
[12] S. Hawking, The unpredictability of quantum gravity, Commun. Math. Phys., $87,395,1982$.

[13] Review of Particle Properties, Particle Data Group, Phys. Rev., D50, 3, 1994.

[14] G.D. Gollin and W.P. Hogan, New Measurements of CP violation parameters as tests of CPT in K meson decay, Proceedings of the 27th International Conference on High Energy Physics, Glasgow, 1994.

[15] G.B. Thomson et al., An experiment studying $K_{L}-K_{S}$ interference to test $C P T$ conservation at the Planck scale, Rutgers preprint RU-96-82.

[16] I.N. Meshkov, Experimental Studies of antihydrogen and positronium physics: Problems and possibilities, Phys. Part. Nucl., 28, 2, 1997.

[17] T.Kinoshita, The fine structure constant, Rep. Prog. Phys., 59, 1459, 1996.

[18] G.W. Erickson and D.R. Yennie, Part I, Ann. Phys., 35, 271, 1965.

[19] G.W. Erickson, Improved Lamb-Shift Calculation for All Values of Z, Phys. Rev. Lett., 27, 12, 1971.

[20] P. Mohr, Lamb Shift in a Strong Coulomb Potential, Phys. Rev. Lett., 34, 16, 1975.

[21] S.G. Karshenboim, The lamb shift in the hydrogen atom, JETP, 79, 2, 1994.

[22] R.T. Robiscoe and T.W. Shyn, Phys. Rev. Lett., 24, 559, 1970.

[23] S.R. Lundeen and F.M. Pipkin, Phys. Rev. Lett., 46, 232, 1981.

[24] S.R. Lundeen, Bull. Amer. Phys. Soc., 20, 11, 1975.

[25] V.G. Pal'chickov, Y.L.Sokolov, and V.P.Yakovlev, Pis'ma Zh. Eksp. Teor. Fiz., 38, 347, 1983 [JETP Lett., 38, 418, 1983]. 
[26] Y.L. Sokolov and V.P.Yakovlev, Measurement of the Lamb shift in the hydrogen atom (n=2), Sov. Phys. JETP., 56, 1, 1982.

[27] D.A. Andrews and G. Newton, Radio-Frequency Atomic Beam Measurement of the $\left(2^{2} S_{1 / 2}, F=0\right)-\left(2^{2} P_{1 / 2}, F=1\right)$ Lamb-Shift Interval in Hydrogen, Phys. Rev. Lett., 37, 19, 1976.

[28] E.W. Hagley and F.M. Pipkin, Separated Oscillatory Field Measurement of Hydrogen $2 S_{1 / 2}-2 P_{3 / 2}$ Fine Structure Interval, Phys. Rev. Lett., 72, 8, 1994.

[29] The Production and Study of Cold Antihydrogen, by the Antihydrogen TRAP Collaboration, G.Gabrielse et al., CERN-SPSLC 96-23/I211, 1996.

[30] Progress report on the design of the ATHENA apparatus, the ATHENA Collaboration, CERN-SPSC 97-9, SPSC/M597, 1997.

[31] D.J. Berkeland et al., Precise optical measurement of Lamb shifts in atomic hydrogen, Phys. Rev. Lett., 75, 13, 1995.

[32] S. Bourzeix et al., High resolution spectroscopy of the hydrogen atom: Determination of the 1S Lamb shift, Phys. Rev. Lett., 76, 3, 1996.

[33] S. Klarsfeld and A. Maquet, Bethe sums for Lamb shift calculations in higher excited states, Phys. Lett. B, 43, 3, 1973.

[34] S. Brodsky and R. Parsons, Precise Theory of the Zeeman Spectrum for Atomic Hydrogen and Deuterium and the Lamb Shift, Phys. Rev., 163, 1, 1967.

[35] M. Charlton et al, Antihydrogen Physics, Phys. Rep., 241, 65, 1994.

[36] L. Brown and G.Gabrielse, Geonium Theory: Physics of a single electron or ion in a Penning trap, Rev. Mod. Phys., 58, 1986.

[37] W. Quint et al., (Anti)hydrogen recombination studies in a nested Penning trap, Hyperfine Interactions, 76, No. 1-4, 181, 1993. 
[38] F.B. Yousif et al., Phys. Rev. Lett., 67, 26, 1991.

[39] H. Bethe and E. Salpeter, Quantum Mechanics of One-and Two-Electron Atoms, Springer-Verlag, Berlin, 1957.

[40] Bertulani and Baur, Electromagnetic Processes in Heavy Ion Collisions, Phys. Rep., 193, 398, 1990.

[41] G.I. Budker and A.N. Shrinsky, Usp. Fiz. Nauk., 124, 561, 1978; [Sov. Phys. Usp., 21, 277, 1978]; M. Bell et al., Nucl. Inst.\& Meth., 190, 237, 1981; H. Poth et al., Z. Phys. A., 332, 171, 1989.

[42] U. Schramm et al., Phys. Rev. Lett., 67, 22, 1991.

[43] A. Wolf, Laser Stimulated Formation and Stabilization of Antihydrogen Atoms, Hyperfine Interactions, 76, No. 1-4, 189, 1993.

[44] B. Deutch et al., Antihydrogen synthesis by the reaction of antiprotons with excited state positronium atoms, Hyperfine Interactions, 76, No. 1-4, 153, 1993.

[45] Y. Ito et al., Possible formation of antihydrogen atoms from metastable antiprotonic helium atoms and positrons/positronium, Hyperfine Interactions, 76, No. $1-4,163,1993$.

[46] M. Iwasaki et al., Phys. Rev. Lett., 67, 1246, 1991.

[47] C.Munger, S.Brodsky, I.Schmidt, Production of relativistic antihydrogen atoms by pair production with positron capture, Phys. Rev., D 49, 7, 1994.

[48] G. Baur, Photon-photon and bremsstrahlung production of fast antihydrogen in $\bar{p}$-nucleus collisions, Phys. Lett., B 311, 343, 1993.

[49] C.Munger, S.Brodsky, I.Schmidt, Production of relativistic antihydrogen atoms by pair production with positron capture and measurement of the Lamb shift, Hyperfine Interactions, 76, 175, 1993. 
[50] S. Brodsky and A.H. Mueller, Using Nuclei to Probe Hadronization in QCD., Phys. Lett., B 206, 4, 1988.

[51] S.J. Brodsky, J.F. Gunion, and D.E. Soper, Physics of heavy-quark production in quantum chromodynamics, Phys. Rev., D 36, 9, 1987.

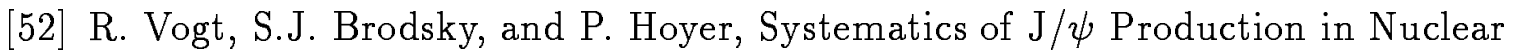
Collisions, Nucl. Phys., B 360, 67, 1991.

[53] G. Baur and Bertulani, C.A., Electromagnetic physics at relativistic heavy ion colliders, for better of for worse, Nucl. Phys., A 505, 835, 1989.

[54] D. Perkins, Introduction to High Energy Physics, third edition, Addison Wesley Publishing Company, Inc., 1987.

[55] G. Baym, Lectures on Quantum Mechanics, 173, Addison-Wesley Publishing Company, Redwood City, CA., 1969.

[56] V.M. Budnev et al., The Two-Photon Particle Production Mechanism. Equivalent Photon Approximation., Phys. Rep., C 15, 4, 1975.

[57] C.A. Bertulani and G. Baur, Antihydrogen production and accuracy of the equivalent approximation, $H E P-P H / 9711273 v 1$ preprint from $L A N L x x x$ archive, 1997; submitted to Phys. Rev. D.

[58] M.J. Rhoades-Brown, C. Bottcher, M.R. Strayer, Feynman-Monte Carlo calculations of electron capture at relativistic energies, Phys. Rev., A 40, No. 5, 2831, 1989.

[59] A.J. Baltz, M.J. Rhoades-Brown, and J. Weneser, Energy Dependence of Bound Electron-Positron Pair Production at Very High Energy Ion-Ion Transits, Phys. Rev., A 44, No. 9, 5569, 1991. 
[60] A. Aste, K. Hencken, D. Trautmann, G. Baur, Electromagnetic pair production with capture, Phys. Rev., A 50, No. 5, 3980, 1994.

[61] U. Becker, Distribution of positrons emitted from pair production with capture in relativistic heavy ion collisions, J. Phys. B, 20,6563, 1987.

[62] C.A. Bertulani and G. Baur, Electromagnetic Processes in Relativistic Heavy Ion Collisions, Nucl. Phys., A 458, 725, 1986.

[63] J. Eichler, Charge Transfer from the Negative Energy Continuum: Alternative Mechanism for Pair Production in Relativistic Atomic Collisions, Phys. Rev. Lett., 75, 20, 1995.

[64] A. Belkacem, et al., Projectile Energy and Atomic Number Dependence of Electron Capture from Pair Production in Relativistic Heavy Ion Collisions, Phys. Rev. Lett., 73, No. 18, 2432, 1994.

[65] A. Belkacem, et al., Measurement of Electron Capture from Electron-Positron Pair Production in Relativistic Heavy Ion Collisions, Phys. Rev. Lett., 71, 10, 1993.

[66] Boero et al., The internal Xe-jet target for the formation of antihydrogen atoms at CERN LEAR, CERN PPE/96-105, 1996, published in Nuovo Cimento A.

[67] Design Report: Tevatron 1 Project, Fermi National Accelerator Laboratory. Batavia, IL, (unpublished), September 1984.

[68] J.Peoples et al., The Fermilab Antiproton Source, IEEE Trans. Nucl. Sci., NS-30, $1970,1983$.

[69] E760 Collaboration, Measurement of the $J / \psi$ and $\psi^{\prime}$ resonance parameters in $\bar{p} p$ annihilation, Phys. Rev., D 47, 3, 1993.

[70] M. Macri, Gas Jet Targets, CERN Accelerator School 1983, CERN 84-15, 1984. 
[71] FNAL P835, Proposal to continue the study of charmonium spectroscopy in $\bar{p}-p$ collisions, 1993.

[72] D. Allspach et al., Refrigerated Hydrogen Gas jet for the Fermilab Antiproton Accumulator, Proceedings of the Cryogenic Engineering Conference, Columbus Ohio, July 17-21, 1995.

[73] A.Cecucci et al., Charmonium Formation in P Anti-P Annihilation by Experiment E760, Nucl. Phys., A 558, 259c, 1993.

[74] S. Trokenheim et al., A $p \bar{p}$ luminosity monitor for Fermilab experiment E760, Nucl. Inst. \& Meth., A 355, 308, 1995.

[75] M.K. Prasad, Acta Physica Polonica, B 10, 635, 1979.

[76] M.S. Gulley et al, Measurement of $H^{-}, H^{\circ}$, and $H^{+}$cross sections produced by foilstripping of $800 \mathrm{MeV} \mathrm{H}^{-}$ions, Phys. Rev., A 53, 3201, 1996.

[77] R.C. Webber and C. Hojvat, Measurement of the Electron Loss for Negative Hydrogen Ions on Carbon at $200 \mathrm{MeV}$., IEEE Trans. Nucl. Sci., NS-26, 3, 4012, 1979.

[78] E. Acerbi et al., Lett. Nuov. Cim., 10, No. 14, 598, 1974.

[79] P.B. Keating et al., Electric-field-induced electron detachment of $800-\mathrm{MeV} H^{-}$ ions, Phys. Rev., A 52, 4547, 1995.

[80] C. Hojvat, M. Joy, and, R.C. Webber, Stripping Foils for Multiturn Charge Exchange Injection into the Fermilab Booster, IEEE Trans. Nucl. Sci., NS-26, 3, 4009, 1979.

[81] D.A. Edwards and M.J. Syphers, An Introduction to the Physics of High Energy Accelerators, John Wiley and Sons, Inc., New York, NY, 1993. 
[82] D.B. Montgomery, Solenoid Magnet Design (The Magnetic and Mechanical Aspects of Resistive and Superconducting Systems), John Wiley and Sons, New York, NY, 1969.

[83] S. Humphries, Charged Particle Accelerators, John Wiley and Sons, New York, NY, 1900.

[84] P.A.M. Dirac, P.A.M., Proc. Camb. Phil. Soc., 26, 361, 1930.

[85] W. Heitler, The Quantum Theory of Radiation, Oxford University Press, London, 1954.

[86] H.W. Kendall and M. Deutsch, Annihilation of Positrons in Flight, Phys. Rev., $101,1,1996$.

[87] W.R. Leo, Techniques for Nuclear and Particle Physics Experiments, SpringerVerlag, 1987.

[88] D.C. Christian et al., High Rate Drift Chambers, Nucl. Inst. \& Meth., A 345, 62, 1994.

[89] G.L. Kane and A. Seidl, Two-body hadron reactions, Rev. Mod. Phys., 48, 2, 1, 1976.

[90] FNAL P862, Proposal for the detection of relativistic antihydrogen atoms produced by pair production with positron capture, 1992.

[91] D.M. Ritson, Techniques of High Energy Physics, Interscience Publishers, Inc., New York, 16, 1961.

[92] W. Nelson, et al., The EGS4 Code System, SLAC-Report-265, 1985.

[93] G.Blanford et al., (Fermilab E862 Collaboration), Observation of Atomic Antihydrogen, submitted to Phys. Rev. Lett. 
[94] V.B. Berestetskii, E.M. Lifshitz, L.P. Pitaevskii, Quantum Electrodynamics, Pergamon Press, 1982. (from Landau and Lifshitz Theoretical Physics series).

[95] M.Mandelkern et al.(Fermilab E862 Collaboration), Letter of intent for a continuation of E862: Measuring the Lamb shift and fine structure of antihydrogen, (unpublished).

[96] R.J. Damburg, V.V. Kolosov, A hydrogen atom in a uniform electric field III, J. Phys., B 12, 16, 1979.

[97] T. Yamabe, A. Tachibana, H. Silverstone, Theory of the ionization of the hydrogen atom by an external electrostatic field, Phys. Rev., A 16, 3, 1977.

[98] W.E. Lamb,Jr., and R.C. Retherford, Phys. Rev., 60, 817, 1941.

[99] V.V. Parkhomchuk, Measurements of the Lamb shift in a relativistic hydrogen atom, Hyperfine Interactions, 44, 315, 1988.

[100] R. Neumann, Fast antihydrogen beam spectroscopy, Hyperfine Interactions, 44, $305,1988$.

[101] G.Blanford et al., (Fermilab E862 Collaboration), Measuring the Antihydrogen Lamb Shift with a Relativistic Antihydrogen Beam, submitted to Phys. Rev. D. 


\section{Appendix A}

\section{Relativistic Cross Section}

\section{Calculation}

This appendix is an attempt to bring together the parts of the calculation needed for the antihydrogen production cross section. The material, adapted from references [94], [47], [85] is not original and is solely intended to condense the material into a readable review format.

\section{A.1 Photoelectric Matrix Element}

The antihydrogen production reaction observed by E862 is based on an antiproton passing near a proton close enough to interact with the proton's electromagnetic near field and produce an $e^{+}-e^{-}$pair. The proton's field can be fourier analyzed into a spectrum of virtual photons. The Weizsächer-Williams method of virtual photons is used to express the virtual photon spectrum as a spectrum of real photons. This is possible because the antiproton's large relativistic boost makes the near field look nearly transverse to the antiproton. Now one can consider the interaction of the antiproton with a single real photon which results in an electron and a bound antihydrogen. One of the insights found in [47] was to change this photon induced capture reaction into the photoelectric reaction by using crossing symmetry $(s \leftrightarrow u)$. So the 
first step is to calculate the photoelectric matrix element. After crossing symmetry is invoked, the Weizsächer-Williams method is used to do the sum over photons in the proton's near field.

For the photoelectric process, the choice of wavefunctions for the initial atom and final electron is important.

$$
M_{p e}(k)=\psi_{f}<\vec{j}_{f i} \cdot \vec{A}>\psi_{i}=\int d^{3} x \psi_{f}^{*}(\vec{\alpha} \cdot \vec{e}) e^{i \vec{k} \cdot \vec{r}} \psi_{i}
$$

The effect of the Coulomb force on both the incoming and outgoing electron (plane wave) is viewed as a perturbation. The initial state wave function is basically a relativistic modification to the non-relativistic hydrogen wave function.

$$
\text { Potential energy : } U=-Z \alpha / r
$$

In order to get the perturbation wave function, The Dirac equation, $(E-U-$ $m \beta+i \vec{\alpha} \cdot \vec{\nabla}) \psi=0$, can be multiplied by its complex conjugate to get a 2 nd order equation, where $\vec{\alpha}, \beta$ are the spin matrices formed from the Dirac matrices,

$$
\begin{array}{r}
(E-U+m \beta-i \vec{\alpha} \cdot \vec{\nabla})(E-U-m \beta+i \vec{\alpha} \cdot \vec{\nabla}) \psi=0, \text { or } \\
\left(\Delta+p^{2}-2 E U\right) \psi=\left(-i \vec{\alpha} \cdot \vec{\nabla} U-U^{2}\right) \psi
\end{array}
$$


The final state wave function of the electron is a sum of plane wave and a perturbation part.

$$
\begin{array}{r}
\psi_{f}=\frac{1}{\sqrt{2 E}}\left[u^{\prime} e^{i \vec{p} \cdot \vec{r}}+\psi^{(1)}\right] \\
\psi_{i}=u \psi_{n o n-r e l}+\psi^{(1)}=\left[1-\frac{i}{2 m} \gamma^{o}(\vec{\gamma} \cdot \vec{\nabla})\right] \frac{u}{\sqrt{2 m}} \psi_{n o n-r e l}, \\
\psi_{n o n-r e l}=\frac{\left(Z e^{2} m\right)^{3 / 2}}{\sqrt{\pi}} e^{-Z e^{2} m r}=R_{o} * Y_{o o}
\end{array}
$$

where $\mathrm{u}=$ spinor part.

The initial state wavefunction is a bound state but has this relativistic modification.

$\left(E^{2}-m^{2}\right)+U^{2}-2 E U+\left(\vec{\alpha}^{2}=1\right) *(\vec{\nabla} \cdot \vec{\nabla})=p^{2}+U^{2}-2 E U+\Delta$

and using,

$$
\{\beta, \vec{\alpha}\}=\beta \vec{\alpha}+\vec{\alpha} \beta=0
$$

and integrating by parts,

$$
\begin{gathered}
\left(\Delta+p^{2}+U^{2}-i \vec{\alpha} \cdot \vec{\nabla} U-2 E U\right) \psi=0 \\
\left(\frac{1}{2 m} \Delta-\left|\epsilon_{n}\right|+\frac{Z \alpha}{r}\right) \psi^{(1)}=\frac{i Z \alpha}{2 m}\left(\vec{\nabla} \frac{1}{r}\right) \cdot(\vec{\alpha} u) \psi_{n o n-r e l}+\ldots
\end{gathered}
$$




$$
\left(\Delta+p^{2}-2 E U\right)\left(\psi=u \psi_{n o n-r e l}+\psi^{(1)}\right)=\left(-i \vec{\alpha} \cdot(\vec{\nabla} U)-U^{2}\right)\left(\psi=u \psi_{n o n-r e l}+\psi^{(1)}\right)
$$

Using,

$$
\frac{p^{2}}{2 m}=-\left|\epsilon_{n}\right|-U
$$

and rejecting terms of order $(Z \alpha)^{2}$,

$$
\psi^{(1)}=-\frac{i}{2 m}(\vec{\alpha} u) \cdot \vec{\nabla} \psi_{n o n-r e l}
$$

Finally with this choice of wave functions, the matrix element becomes

$$
\begin{aligned}
M_{f i}= & \frac{1}{\sqrt{2 m}} \frac{1}{\sqrt{2 E}} \cdot \int d^{3} x \bar{u}^{\prime}(\vec{\gamma} \cdot \vec{e}) \cdot\left[1-\frac{i}{2 m} \gamma^{o}(\vec{\gamma} \cdot \vec{\nabla})\right] . \\
& \cdot u \psi_{\text {non-rel }} \cdot e^{-i(\vec{p}-\vec{k}) \cdot \vec{r}}+\bar{\psi}^{(1)}(\vec{\gamma} \cdot \vec{e}) e^{i \vec{k} \cdot \vec{r}} u \psi_{\text {non-rel }}
\end{aligned}
$$

Now integrate by parts (using the surface boundary condition, $\int_{S} \partial\left(d^{3} x\right) u \psi_{n o n-r e l} e^{-i(\vec{p}-\vec{k}) \cdot \vec{r}}=0$ where $\mathrm{S}$ is sphere with $\left.r \rightarrow \infty\right)$,

$$
\int(\cdot \vec{\nabla}) u \psi_{n o n-r e l} e^{-i(\vec{p}-\vec{k}) \cdot \vec{r}} d^{3} x
$$

$$
M_{f i}=\frac{\left(Z e^{2} m\right)^{3 / 2}}{2 \sqrt{\pi m E}} \bar{u}^{\prime}(\vec{\gamma} \cdot \vec{e})\left[1+\frac{1}{2 m} \gamma^{o} \vec{\gamma} \cdot(\vec{p}-\vec{k})\right] u F_{(\vec{p}-\vec{k})}\left(e^{-Z e^{2} m r}\right)+\bar{\psi}_{-\vec{k}}^{(1)}(\vec{\gamma} \cdot \vec{e}) u
$$

where the non-relativistic wave function has been replaced by a constant but only in the last term (not inside the derivative). 
The Fourier transforms needed are,

$$
\begin{aligned}
F_{(\vec{p}-\vec{k})}\left(e^{-Z e^{2} m r}\right) & =\frac{8 \pi Z e^{2} m}{(\vec{p}-\vec{k})^{4}} \\
F_{(\vec{p}-\vec{k})}(1 / r) & =\frac{4 \pi}{(\vec{k}-\vec{p})^{2}}
\end{aligned}
$$

To calculate $\bar{\psi}_{-\vec{k}}^{(1)}$, the Dirac equation is used, 


$$
\left(\gamma^{o} E+i \vec{\gamma} \cdot \vec{\nabla}-m\right) \psi^{(1)}=e\left(\vec{\gamma}^{\mu} A_{\mu}\right) u^{\prime} e^{i p \cdot \vec{r}}=-\frac{Z e^{2}}{r} \gamma^{o} u^{\prime} e^{i p \cdot \vec{r}}
$$

and

$$
\begin{array}{r}
\int\left(\gamma^{o} E+i \vec{\gamma} \cdot \vec{\nabla}+m\right)\left(\gamma^{o} E+i \vec{\gamma} \cdot \vec{\nabla}-m\right) \psi^{(1)} e^{-i \vec{k} \cdot \vec{r}} d^{3} x \\
=-Z e^{2} \int\left(\gamma^{o} E+i \vec{\gamma} \cdot \vec{\nabla}+m\right) \gamma^{o} u^{\prime} \frac{1}{r} e^{i p \cdot \vec{r}} e^{-i \vec{k} \cdot \vec{r}} d^{3} x \\
=\int\left(\Delta+p^{2}\right) \psi^{(1)} e^{-i \vec{k} \cdot \vec{r}} d^{3} x
\end{array}
$$

using $\gamma^{\circ}, \vec{\gamma}=0$ and a parts integration,

$$
\begin{gathered}
\vec{\gamma} \cdot \int \vec{\nabla} \frac{1}{r} e^{i(\vec{p}-\vec{k}) \cdot \vec{r}} d^{3} x=-\vec{\gamma} \cdot F_{(\vec{k}-\vec{p})}\left(\frac{1}{r}\right)(-1)(-k) e^{i(\vec{p}-\vec{k}) \cdot \vec{r}} \\
\int \Delta \psi^{(1)} e^{-i k \cdot \vec{r}} d^{3} x=-k^{2} \psi_{\vec{k}}^{(1)} \\
\left(p^{2}-k^{2}\right) \psi_{k}^{(1)}=-Z e^{2} \int\left[2 E \gamma^{o}+\vec{\gamma} \cdot(\vec{p}-\vec{k})\right] F_{(\vec{k}-\vec{p})}\left(\frac{1}{r}\right) e^{i(\vec{p}-\vec{k}) \cdot \vec{r}} \gamma^{o} u^{\prime} d^{3} x
\end{gathered}
$$

using $m=\left(\gamma^{\circ} E+\vec{p} \cdot \vec{\gamma}\right) \gamma^{\circ} u^{\prime}$,

Resulting in:

$$
\bar{\psi}_{-\vec{k}}^{(1)}=\left[\frac{4 \pi}{(\vec{k}-\vec{p})^{2}} \frac{Z e^{2}}{\left(k^{2}-p^{2}\right)} \bar{u}^{\prime}\left(2 E \gamma^{o}+\vec{\gamma} \cdot(\vec{k}-\vec{p})\right)\right] \gamma^{o}
$$




$$
\begin{array}{r}
M_{f i}=\frac{\left(Z e^{2} m\right)^{3 / 2}}{2 \sqrt{\pi m E}} \bar{u}^{\prime}\left[1+\frac{1}{2 m} \gamma^{o} \vec{\gamma} \cdot(\vec{p}-\vec{k})\right] * u \frac{8 \pi Z e^{2} m}{(\vec{p}-\vec{k})^{4}} \\
+4 \pi Z e^{2} \bar{u}^{\prime} \frac{2 E \gamma^{o}+\vec{\gamma} \cdot(\vec{k}-\vec{p})}{\left(k^{2}-p^{2}\right)(\vec{k}-\vec{p})^{2}} * \gamma^{o}(\vec{\gamma} \cdot \vec{e}) u \\
M_{f i}=\frac{4 \pi\left(Z e^{2} m\right)^{5 / 2}}{\sqrt{m E}(\vec{k}-\vec{p})^{2}} \bar{u}^{\prime} A u
\end{array}
$$

$$
\begin{array}{r}
A=a(\vec{\gamma} \cdot \vec{e})+(\vec{\gamma} \cdot \vec{e}) \gamma^{o}(\vec{\gamma} \cdot \vec{b})+(\vec{\gamma} \cdot \vec{c}) \gamma^{o}(\vec{\gamma} \cdot \vec{e}) \\
a \equiv \frac{1}{(\vec{k}-\vec{p})^{2}}+\frac{E}{m} \frac{1}{k^{2}-p^{2}} \\
\vec{b} \equiv \frac{-(\vec{k}-\vec{p})}{2 m(\vec{k}-\vec{p})^{2}} \\
\vec{c} \equiv \frac{-(\vec{k}-\vec{p})}{2 m\left(k^{2}-p^{2}\right)}
\end{array}
$$

The photoelectric matrix element can now be written more compactly using the matrix A defined in [94] and its Hermitian conjugate, $A^{\dagger}$ :

$$
\begin{gathered}
\left|M_{f i}\right|_{p e}^{2}=\frac{16 \pi\left(Z e^{2} m\right)^{5}}{m \varepsilon(\vec{k}-\vec{p})^{4}}\left(\bar{u}^{\prime} A u\right)\left(\bar{u} \bar{A} u^{\prime}\right), \bar{A} \equiv \gamma^{o} A^{\dagger} \gamma^{o} \\
d \sigma=\frac{8 e^{2}\left(Z e^{2} m\right)^{5}}{\omega(\vec{k}-\vec{p})^{4}} \frac{|\vec{p}|}{m}\left(\bar{u}^{\prime} A u \bar{u} \bar{A} u^{\prime}\right) d \Omega \\
d \sigma=\frac{1}{2} e^{2} \pi|\vec{p}|\left|M_{f i}\right|^{2} d \Omega
\end{gathered}
$$




$$
\frac{16 e^{2}\left(Z e^{2} m\right)^{5}|\vec{p}|}{m \omega(\vec{k}-\vec{p})^{4}} \operatorname{Tr}\left(\rho^{\prime} A \rho \bar{A}\right) d \Omega
$$

where $\rho$ is the initial polarization matrix, $\rho^{\prime}$ is the final state polarization matrix, the first averaged, the second summed over spin directions.

$$
\begin{gathered}
\rho=\frac{1}{2} m\left(\gamma^{o}+1\right)=\frac{1}{2}(\gamma \cdot p+m) \\
\rho^{\prime}=\frac{1}{2}\left(\gamma^{\circ} \varepsilon-\vec{\gamma} \cdot \vec{p}+m\right)=\frac{1}{2}(\gamma \cdot p+m)\left(1-\gamma^{5} \vec{\gamma} \cdot \vec{g}\right)
\end{gathered}
$$

where $\vec{g}$ is the spin in the rest frame.

$$
\begin{array}{r}
\vec{\gamma}^{\dagger}=-\vec{\gamma}, \gamma^{o \dagger}=\gamma^{o},\left\{\gamma^{o}, \vec{\gamma}\right\} \equiv \gamma^{o} \vec{\gamma}+\vec{\gamma} \gamma^{o}=0 \\
\operatorname{Tr}\left(\rho^{\prime} A \rho \bar{A}\right)=\operatorname{Tr}\left\{\frac { 1 } { 2 } ( \gamma ^ { o } \varepsilon - \vec { \gamma } \cdot \vec { p } + m ) \left[a(\vec{\gamma} \cdot \vec{e})+(\vec{\gamma} \cdot \vec{e}) \gamma^{o}(\vec{\gamma} \cdot \vec{b})+\right.\right. \\
\left.(\vec{\gamma} \cdot \vec{c}) \gamma^{o}(\vec{\gamma} \cdot \vec{e})\right] \cdot \frac{1}{2 m}\left(\gamma^{o}+1\right)\left[a \gamma^{o}(\vec{\gamma} \cdot \vec{e})^{\dagger} \gamma^{o}+\right. \\
\left.\left.\gamma^{o}\left\{(\vec{\gamma} \cdot \vec{e}) \gamma^{o}(\vec{\gamma} \cdot \vec{b})\right\}^{\dagger} \gamma^{o}+\gamma^{o}\left\{(\vec{\gamma} \cdot \vec{c}) \gamma^{o}(\vec{\gamma} \cdot \vec{e})\right\}^{\dagger} \gamma^{o}\right]\right\}
\end{array}
$$

Using,

$$
\begin{array}{r}
\frac{1}{4} \operatorname{Tr}((\vec{A} \cdot \vec{\gamma})(\vec{B} \cdot \vec{\gamma}))=-\vec{A} \cdot \vec{B} \\
\frac{1}{4} \operatorname{Tr}((\vec{A} \cdot \vec{\gamma})(\vec{B} \cdot \vec{\gamma})(\vec{C} \cdot \vec{\gamma})(\vec{D} \cdot \vec{\gamma}))= \\
=(\vec{A} \cdot \vec{B})(\vec{C} \cdot \vec{D})-(\vec{A} \cdot \vec{C})(\vec{B} \cdot \vec{D})+(\vec{A} \cdot \vec{D})(\vec{B} \cdot \vec{C})
\end{array}
$$


After three pages of algebra,

$$
\operatorname{Tr}\left(\rho^{\prime} A \rho \bar{A}\right)=\frac{m}{\varepsilon+m}[a \vec{p}-(\vec{b}-\vec{c})(\varepsilon+m)]^{2}+4 m(\vec{b} \cdot \vec{e})[(\varepsilon+m)(\vec{c} \cdot \vec{e})+a(\vec{p} \cdot \vec{e})]
$$

$\vec{e}$ is real and linear-polarized. $\left(\hat{e}=\hat{e}^{*}\right)$

$$
\begin{array}{r}
\operatorname{Tr}\left(\rho^{\prime} A \rho \bar{A}\right)=\frac{m}{\varepsilon+m}\left[a^{2}(\vec{p} \cdot \vec{p})-2 a \vec{p} \cdot(\vec{b}-\vec{c})(\varepsilon+m)+\right. \\
\left.+\left(b^{2}+c^{2}-2 \vec{b} \cdot \vec{c}(\varepsilon+m)^{2}\right)\right] \\
+4 m(\vec{b} \cdot \vec{e})(\varepsilon+m)(\vec{c} \cdot \vec{e})+4 m(\vec{b} \cdot \vec{e}) a(\vec{p} \cdot \vec{e})
\end{array}
$$

Now let's assume a right-handed, spherical coordinate system:

$$
\begin{gathered}
(\vec{k} \cdot \vec{p})^{2}=k^{2} p^{2} \cos ^{2} \theta \\
(\vec{p} \cdot \hat{e})^{2}=p^{2} \sin ^{2} \theta \cos ^{2} \phi \\
(\vec{k} \cdot \hat{e})=0 \\
\operatorname{Tr}\left(\rho^{\prime} A \rho \bar{A}\right)=\left[\frac{a^{2} p^{2} m}{\varepsilon+m}+a\left(\vec{k} \cdot \vec{p}-p^{2}\right)\left(\frac{1}{k^{2}-p^{2}}-\frac{1}{(\vec{k}-\vec{p})^{2}}\right)\right. \\
+\frac{\varepsilon+m}{4 m}(\vec{k}-\vec{p})^{2}\left(\frac{1}{k^{2}-p^{2}}-\frac{1}{(\vec{k}-\vec{p})^{2}}\right)^{2} \\
\left.-\frac{\varepsilon+m}{2 m} \frac{k^{2} p^{2}-(\vec{k} \cdot \vec{p})^{2}}{\left(k^{2}-p^{2}\right)(\vec{k}-\vec{p})^{2} k^{2}}+a \frac{k^{2} p^{2}-(\vec{k} \cdot \vec{p})^{2}}{(\vec{k}-\vec{p})^{2} k^{2}}\right] \\
|M|_{p h o t o}^{2}=\frac{(32 \pi)^{2} \alpha^{6} m^{4} M^{2}}{(\vec{k}-\vec{p})^{4}} \operatorname{Tr}\left(\rho^{\prime} A \rho \bar{A}\right)
\end{gathered}
$$




\section{A.2 Cross Section Integration}

The next step is to express the kinematic variables of the photoionization process in terms of the Mandelstam variables. We need $k^{2}, p^{2}$, and $\vec{k} \cdot \vec{p}$ as functions of $\mathrm{s}, \mathrm{t}, \mathrm{u}$. Notice that if one switches the definitions of $t$ and $u$, the variables represent instead the

capture process. Assume $\mathrm{m}=$ electron mass and $\mathrm{M}=$ proton mass. $\varepsilon=\sqrt{|\vec{p}|^{2}+m^{2}}$.

$$
\begin{array}{r}
s=M^{2}+2 M \omega \\
t=M^{2}-2 \varepsilon M+m^{2} \\
u=m^{2}-2 \omega \varepsilon+2 \vec{k} \cdot \vec{p}
\end{array}
$$

Now we would like the capture cross section, of course, so we use the crossing symmetry by switching the variables: $(s \leftrightarrow t)$. Notice in Munger, Brodsky, and Schmidt [47], they use the Mandelstam variables for the capture process instead but interchange $s \leftrightarrow u$. This turns out to yield the same cross section. An equivalent way of doing the transformation is not to use the Mandelstam variables at all but simply to switch the sign of the electron energy $\epsilon \leftrightarrow-\epsilon$. To reiterate the definitions shown 
in section 1.3 originally from [47],

$$
\begin{gathered}
\frac{\alpha}{\pi} * \bar{\sigma}=\int_{2 m_{e}}^{M_{\bar{p} \gamma}} \frac{d \omega}{\omega} \sigma_{\gamma \bar{p} \rightarrow \bar{H}(1 S) e^{-}}(\omega) \\
\sigma_{\gamma^{*} \bar{p} \rightarrow e^{-} \bar{H}(1 S)}(\omega)=\frac{p}{64 \pi^{2} \omega M^{2}} \int|M|_{\text {capture }}^{2} d \Omega_{e}
\end{gathered}
$$

The ratio of $\bar{\sigma}$ (resulting from the $s \leftrightarrow t$ ) to $\bar{\sigma}$ (from the $\epsilon \rightarrow \epsilon$ method) is shown in figure A.1 along with the individual cross section estimates found by integrating equation A.59. The horizontal axis is the upper limit of the photon energy integration in units of the electron mass. The upper limits for the integrations over transverse momentum transfer, $q_{\perp}^{2}$, and over photon energy, $\omega$, are important choices. The suggestion from [47] is to integrate up to $q_{\perp}^{2}=\omega^{2}$ and then integrate $\omega$ up to $M_{p} * \gamma=$ $5500 * 2 m_{e}$ at $6 \mathrm{GeV} / \mathrm{c}$, for example. 

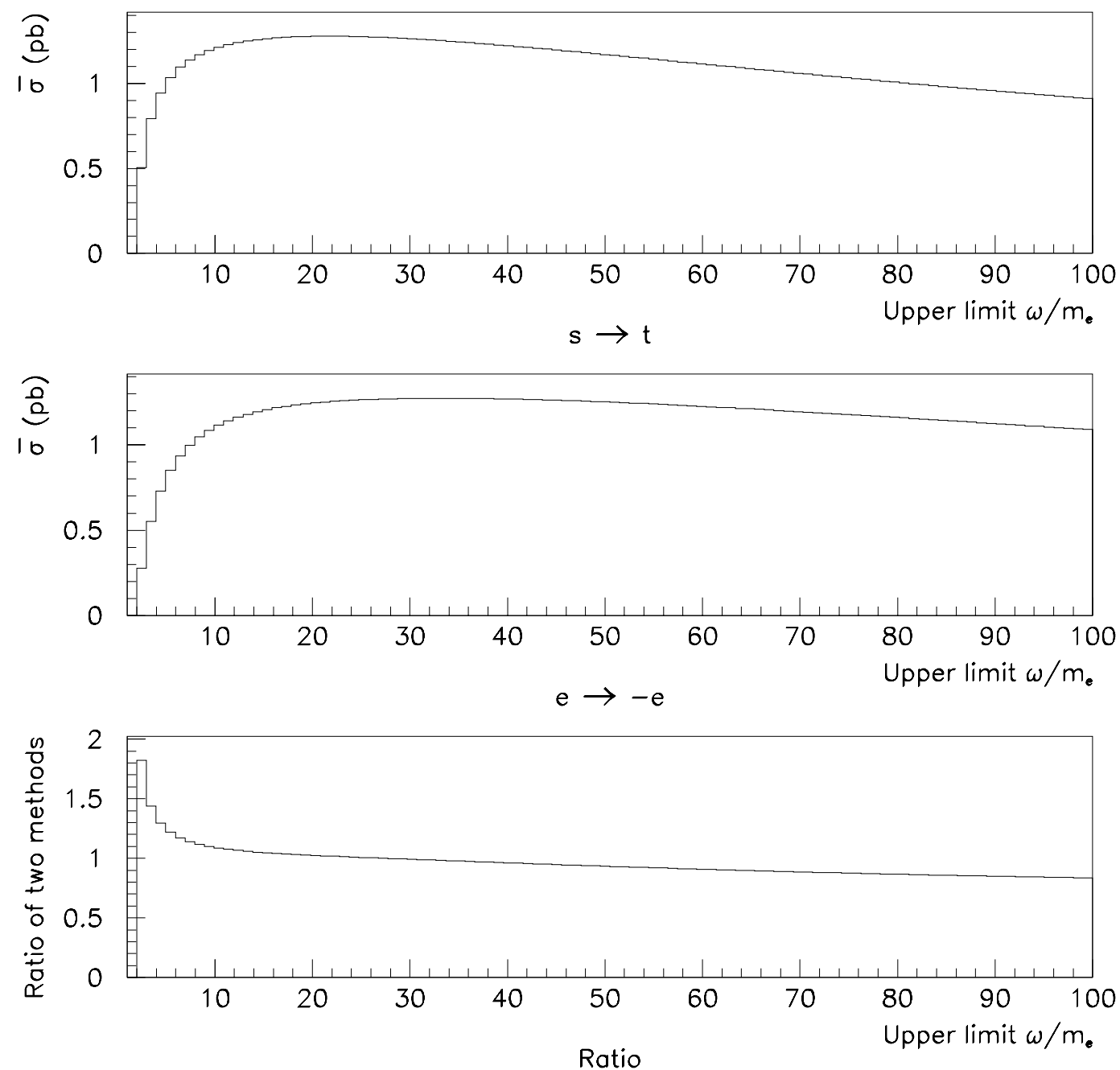

Figure A.1: Results of integrating capture cross section formula shown in equation A.59, (derived in[47]), using two different transformations for the crossing symmetry. 


\section{Appendix B}

\section{Spectroscopy of Relativistic $\bar{H}$}

\section{B.1 Goals}

In order to explore the possibility of a CPT symmetry violation in the hydrogenantihydrogen system, the energy levels of antihydrogen atoms need to be measured precisely and compared to those of hydrogen atoms. Antihydrogen atoms produced with the technique described in this dissertation, besides having a small production rate, are characterized by large energy spreads making it difficult to precisely measure the energy spectrum. One can partially circumvent this by designing an experiment which exploits the interference of a two level quantum system. The level splittings are really what we want to measure in order to learn about the interactions of the constituent particles as opposed to the individual particle masses. An overall, uniform shift of all of the antihydrogen energy levels with respect to those of hydrogen would be missed using this method.

I will outline, in the subsequent chapter, plans for measuring the fine structure and Lamb shift splittings of the $n=2$ level using an interference method by extending the present experiment. A discussion of similar methods is given in [16]. The first 
step is to increase the production rate in order to make higher statistics spectroscopic measurements. Next, it will be shown that antihydrogen $n>1$ states can be produced (with an excitation foil) and measured as distinct from the $\mathrm{n}=1$ states. Finally, methods of exciting the $2 S_{1 / 2}-2 P_{1 / 2}$ transition and measuring the energy difference are discussed. A more detailed description is found in [95].

\section{B.2 Unperturbed Spectrum}

The unperturbed spectrum of the hydrogen atom is shown in figure B.1. A hierarchy of three types of energy splittings dominate the corrections to the $-1 / n^{2}$ energy dependence of the Bohr levels. These are the spin-orbit, Lamb shift, and hyperfine corrections. The $2 P_{1 / 2}$ and $2 P_{3 / 2}$ states decay by a fast electric dipole transition to the ground state $(\tau=1.6 \mathrm{~ns})$.

\section{B.3 Increasing the Production Rate}

One of the easy ways to increase the antihydrogen production rate exploits the $Z^{2}$ dependence of the cross section where $\mathrm{Z}$ is the atomic number of the target nuclei. Various heavy gases have been considered for use in the jet target, (described in section 2.2).

Only a hydrogen jet target is conducive to the study of charmonium resonances (E835), (see section 2.3) since the spread in reconstructed energy of the resonances 


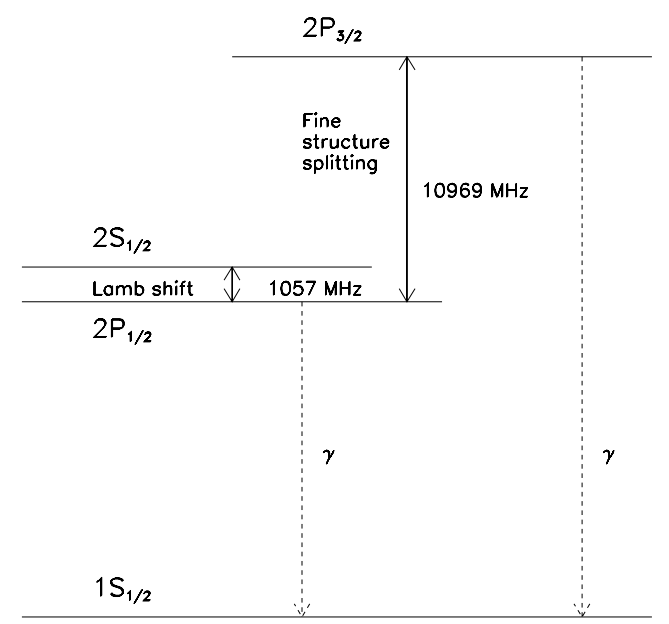

Figure B.1: The hydrogen spectrum in zero field.

becomes too large. The main problem for an antihydrogen experiment is that the beam emittance increases due to Coulomb scattering and nuclear scattering.

Taking data in a dedicated session would allow the experiment to be done at the injection momentum $(8.85 \mathrm{GeV} / \mathrm{c})$. Less beam manipulation is necessary in this case. The beam is well understood at the injection momentum and the angular divergences are smaller. Running the experiment parastically to an E835 successor experiment, though, would imply beam momenta clustered near $3.5 \mathrm{GeV} / \mathrm{c}$ and $6 \mathrm{GeV} / \mathrm{c}$ would be chosen. The following discussion assumes running at injection momentum exclusively.

The gas jet target tested at LEAR for PS210 is very similar to the one used here. It was constructed by the same group and has been used with neon and xenon gas[66]. The main problem was the large increase in Coulomb scattering which scales 
as $Z^{2}$. The FNAL antiproton source cooling systems are more powerful but they are operating at close to their limit at current luminosities [71]. The cooling cannot correct the large angle nuclear scattering since it falls outside the angular acceptance of the pickups. Although it does not increase as fast $\left(\sim A^{2 / 3}\right)$ with larger $\mathrm{Z}$ as the Coulomb scattering, it will affect the beam lifetime.

\section{B.4 Effect of Magnetic Fields}

In the relativistic antihydrogen rest frame, laboratory magnetic fields Lorentz transform into very large electric fields. For a 1 Tesla magnet at injection energy $(\gamma \sim 9)$, the electric field has the value, $E=\beta \gamma c B=2.7 \times 10^{7} \mathrm{~V} / \mathrm{cm}$. This is $0.5 \%$ of the atomic electric field at the 1 st Bohr orbit $\left(5.14 \times 10^{9} \mathrm{~V} / \mathrm{cm}\right)$. This clearly breaks the spherical symmetry of the hydrogen atom. Derivations of the atomic wavefunctions use the expansion in parabolic coordinates, since one symmetry axis (that of the applied electric field) is favored [39]. The perturbations to the energy levels and wave functions have been calculated [96]. The ionization rate calculated using second order perturbation theory is shown in equation B.1[97]. A quadratic correction to this equation can be found in [96]. It is highly sensitive to the applied field because of the exponential factor:

$$
R=\frac{1}{n^{3} n_{2} !\left(n_{2}+|m| !\right)}\left(\frac{n^{3}|\vec{E}|}{4}\right)^{n-2 n_{2}-|m|-1} e^{3\left(n-2 n_{2}-|m|\right)-2 /\left(3 n^{3}|\vec{E}|\right)} \frac{\alpha^{2} m c^{2}}{\hbar}
$$

The parabolic quantum numbers $\left(n, n_{1}, n_{2}, m\right)$ are: $n=$ principal quantum number, $m=$ magnetic quantum number, $n_{1}-n_{2}$ characterizes the distortion of the previously spherical wavefunction due to a given applied field. 


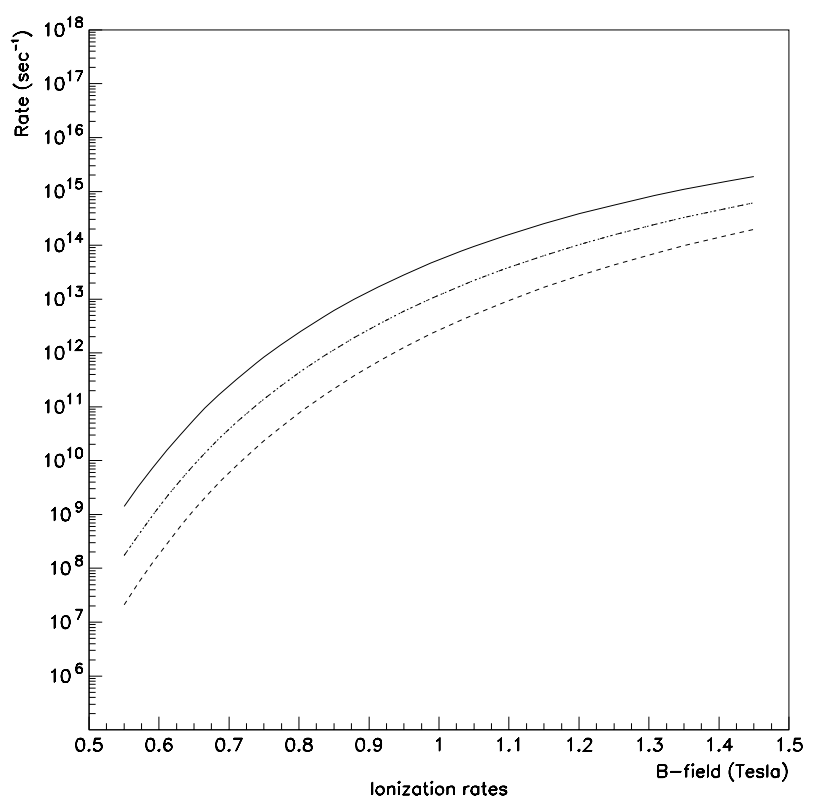

Figure B.2: Hydrogen ionization rates in magnetic fields for $\gamma=9$.

In addition to the large ionization rate, the electric field causes a large Stark splitting of the energies of the nearly degenerate states $\left(2 S_{1 / 2}, 2 P_{1 / 2}, 2 P_{3 / 2}\right)$. Instead, three new states exist (denoted short,medium,long) corresponding to their respective lifetimes for ionization.

\section{B.5 Observing $n>1$ States}

The $n>1$ antihydrogen atoms ionize while traversing the first bend dipole. Only $\mathrm{n}=1$ atoms are left and any $\mathrm{n}>1$ atoms must be created by excitation from the ground state. A very thin carbon foil $\left(\sim 70 \mu \mathrm{g} / \mathrm{cm}^{2}\right)$ will yield $10 \%$ of the atoms in the $\mathrm{n}=2$ 
states as well as another $10 \%$ of the original number being ionized. The rest travel through the foil as $n=1$ atoms.

One scheme for separating the different principal quantum number states expands on [47]. An excitation foil is placed upstream of the present E862 apparatus, (see section 3.1 ), within a bend magnet. The bend magnet ionizes the $\mathrm{n}>1$ states. The remaining magnetic field bends the resulting antiprotons an amount proportional to the length of magnetic field encountered. The bend length in the magnets is adjusted so that there are distinct antiproton spots in the last wire chamber corresponding to the $n=1$ and $n=2$ atoms. The original E862 thicker foil ionizes the remaining $n=1$ atoms and detects the ground state ionization remnants. To detect the positron from the $\mathrm{n}=2$ atoms, scintillators would be placed a distance $\mathrm{D}$ away from the beam where $\mathrm{D}$ is the expected diameter of a positron's path in the magnetic field. In this way, one can measure the longitudinal position of the ionization vertex by extrapolating the positron track backward. Present designs favor either scintillating fiber arrays, a wall of silicon (300 $\mu \mathrm{m}$ thick), or straw tubes [95]. One can measure the vertex of the ionization and then count states of $n=2$ multiplet vs $n=1$.

\section{B.6 Measuring a Lamb Shift in antihydrogen}

Observation of the $\left(2 S_{1 / 2}-2 P_{1 / 2}\right)$ Lamb shift splitting in hydrogen $(\mathrm{f}=1057$ $\mathrm{MHz} ; \Delta \mathrm{E}=4.4 \mu \mathrm{eV}$ ) was one of the first direct tests of quantum electrodynamics [98]. To measure the Lamb shift, the anti-atoms must first be excited to the $n=2$ states, as discussed in the previous section B.5. 
Different methods to excite the $2 \mathrm{~S}-2 \mathrm{P}$ transition have been proposed. In one method, the atoms would traverse an RF cavity. They would be followed by a $\gamma(1+$ $\beta) f=18 \mathrm{GHz}$ (for $\gamma=9$ ) travelling wave which would excite the $2 \mathrm{~S}-2 \mathrm{P}$ transition. Since the $2 \mathrm{P}$ state decays quickly, (in $1.6 \mathrm{~ns}$ ), to the ground state, those $2 \mathrm{~S}$ atoms which are excited to the $2 \mathrm{P}$ state would decay to the $1 \mathrm{~S}$ state over a distance of 215 $\mathrm{cm}$. A decrease in the $2 \mathrm{~S}$ population would be observed at the resonant transition frequency. Afterwards, they would traverse a high magnetic field (2 Tesla) region. The Stark electric field seen in the $\bar{H}$ rest frame would ionize $\mathrm{n}=2$ atoms in a mean distance of $10 \mathrm{~cm}$ but would not ionize the $\mathrm{n}=1$ atoms, so the $2 \mathrm{~S}$ states can be detected and counted. If the cavity is off resonance, all the original $n=2$ atoms are counted. At lower beam energies, a static electric field can be used [26],[99].

Another way of accomplishing a Lamb shift measurement is by exploiting the Lorentz boosted magnetic fields. Two magnets with magnetic fields just less than 1 Tesla are used, (see figure B.3). The first magnet Stark shifts the almost degenerate $\mathrm{n}=2$ multiplet into linear combinations of the unperturbed states $2 S_{1 / 2}, 2 P_{1 / 2}$, and $2 P_{3 / 2},\left(n L_{J}\right)$. They have different lifetimes in the field region in a 25:5:1 ratio for one choice of field (0.800 Tesla), for example. The magnet is constructed to be long enough to ionize the short and medium-lived states while having minimal effect on the longlived states. When the magnetic field fringe region is contained within a very short distance, the changes to the states can be considered non-adiabatic and the states become instant linear combinations of the unperturbed (zero field) eigenstates.

$$
\begin{array}{r}
\Psi(\text { long })=\sqrt{\frac{1}{2}}\left|S_{1 / 2}\right\rangle+\sqrt{\frac{1}{6}}\left|P_{1 / 2}\right\rangle-\sqrt{\frac{1}{3}}\left|P_{3 / 2}\right\rangle \\
\Psi(\text { medium }, a)=\left|P_{3 / 2}\right\rangle
\end{array}
$$




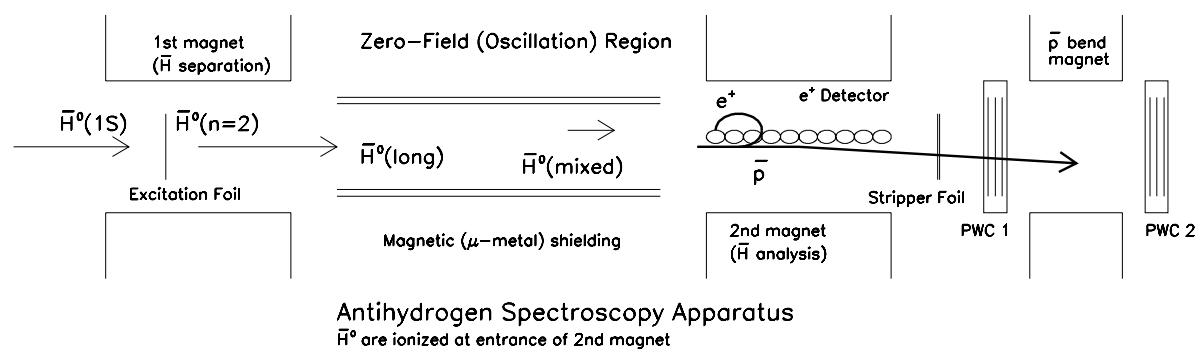

Figure B.3: Experimental apparatus needed to measure Lamb shift.

$$
\begin{array}{r}
\Psi(\text { medium }, b)=\sqrt{\frac{1}{3}}\left|P_{3 / 2}\right\rangle+\sqrt{\frac{2}{3}}\left|P_{1 / 2}\right\rangle \\
\Psi(\text { short })=\sqrt{\frac{1}{2}}\left|S_{1 / 2}\right\rangle-\sqrt{\frac{1}{6}}\left|P_{1 / 2}\right\rangle+\sqrt{\frac{1}{3}}\left|P_{3 / 2}\right\rangle
\end{array}
$$

The original state evolves to a linear combination of long, short, and mediumlived states, basically regenerating short and medium states. The probability of finding the (anti)hydrogen in one of the (short, medium, long) states is characterized by a longitudinal interference pattern, (see figure B.4). The period of the oscillation depends upon the Lamb shift and the fine structure energy splittings and not on the magnetic fields used. The precision of measuring the period of oscillation depends on the amplitudes and thus does depend on the experimental parameters such as field strengths. This phenomenon is similar to the interference seen in the neutral $\mathrm{K}$ meson system [55]. There is also a slow exponential decay superimposed on the oscillation pattern due to the $2 P \rightarrow 1 S$ decays. The decay length for these new states in zero field is now twice the original $2 \mathrm{P}$ decay length $(430 \mathrm{~cm}=2 * 215 \mathrm{~cm}$ at $8.85 \mathrm{GeV} / \mathrm{c}$ ), an interesting by-product of the mixing [16]. By placing the second 
magnet at different points along the $\bar{H}^{\circ}$ direction of travel, different parts of the interference pattern can be observed. The antiprotons from the different states will bend in the remaining $\int B d L$ and result in a sum of exponential distributions in the wire chamber bend plane direction. The positron detector will help determine the ionization vertex as described sectionsec:obsern1. The particular quantum state (long, medium, short) at the time of the ionization can be identified with the antiproton and positron information. More detail can be found in [101] and [95]. 


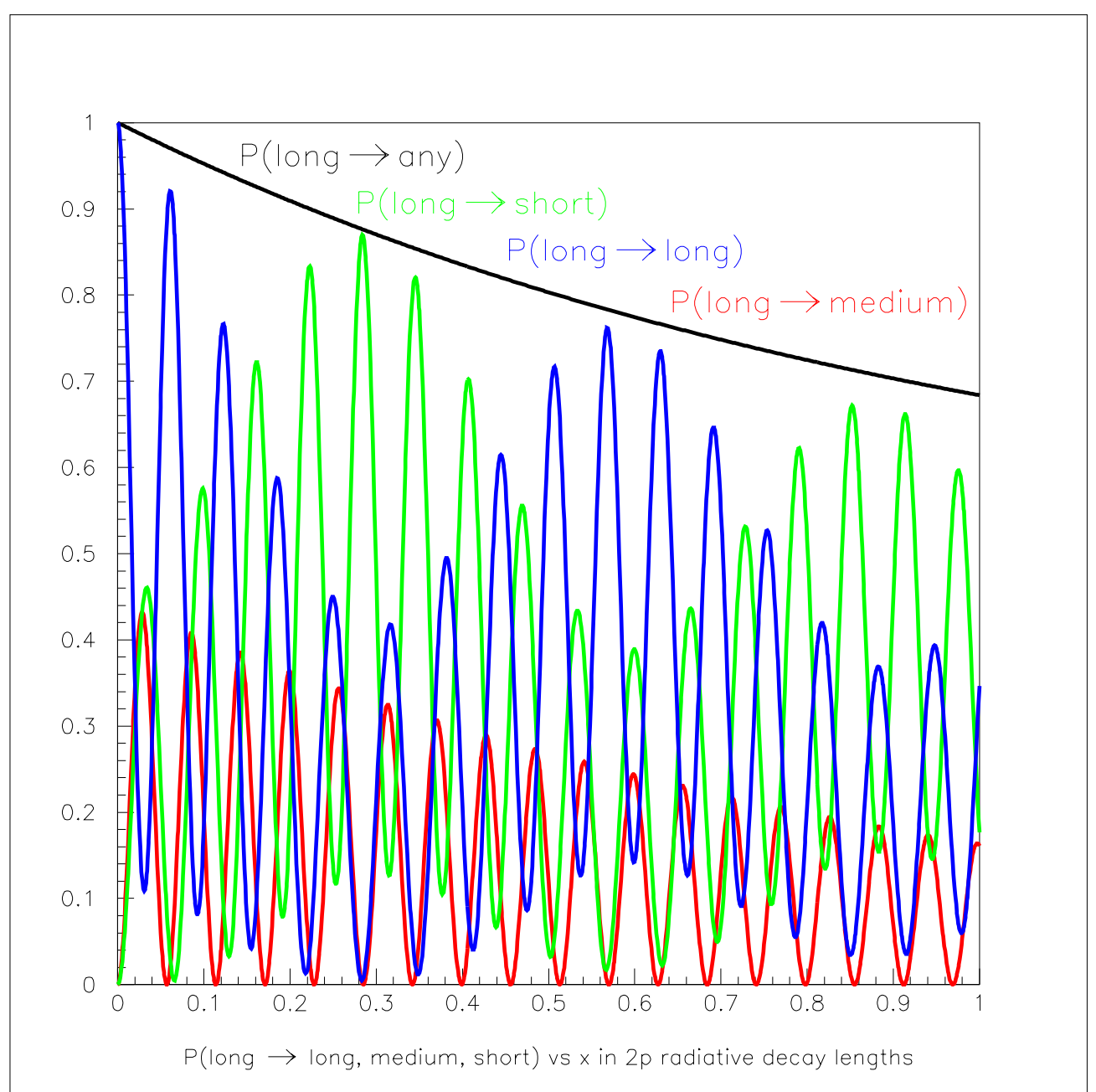

Figure B.4: Oscillatory probabilities for existing in the $n=2$ antihydrogen states (long,medium,short) within the zero field region as a function of flight distance. 